\title{
Refining natural killer cell-based immunotherapy
}

Citation for published version (APA):

Mahaweni - van Eijl, N. M. (2018). Refining natural killer cell-based immunotherapy: Strategies to unleash the killer in a suppressive tumor microenvironment . [Doctoral Thesis, Maastricht University]. ProefschriftMaken Maastricht. https://doi.org/10.26481/dis.20181219nm

Document status and date:

Published: 01/01/2018

DOI:

10.26481/dis.20181219nm

Document Version:

Publisher's PDF, also known as Version of record

\section{Please check the document version of this publication:}

- A submitted manuscript is the version of the article upon submission and before peer-review. There can be important differences between the submitted version and the official published version of record.

People interested in the research are advised to contact the author for the final version of the publication, or visit the DOI to the publisher's website.

- The final author version and the galley proof are versions of the publication after peer review.

- The final published version features the final layout of the paper including the volume, issue and page numbers.

Link to publication

\footnotetext{
General rights rights.

- You may freely distribute the URL identifying the publication in the public portal. please follow below link for the End User Agreement:

www.umlib.nl/taverne-license

Take down policy

If you believe that this document breaches copyright please contact us at:

repository@maastrichtuniversity.nl

providing details and we will investigate your claim.
}

Copyright and moral rights for the publications made accessible in the public portal are retained by the authors and/or other copyright owners and it is a condition of accessing publications that users recognise and abide by the legal requirements associated with these

- Users may download and print one copy of any publication from the public portal for the purpose of private study or research.

- You may not further distribute the material or use it for any profit-making activity or commercial gain

If the publication is distributed under the terms of Article $25 \mathrm{fa}$ of the Dutch Copyright Act, indicated by the "Taverne" license above, 


\section{Refining natural killer cell-based immunotherapy}

Strategies to unleash the killer in a suppressive tumor microenvironment

Niken Miranti Mahaweni 
๑) Niken Miranti Mahaweni, 2018. Maastricht, The Netherlands.

All rights reserved. No part of this publication may be reproduced, stored in a retrieval system of any nature, or transmitted, in any form or by any means (electronic, mechanical, photocopying, recording or otherwise) without written permission of the author or when appropriate, by the publisher holding the copyright of the published article.

ISBN:

978-94-6380-136-2.

Cover art: $\quad$ Niken Miranti Mahaweni /image source: www.vectorstock.com

Layout \& printing: proefschriftmaken.nl

The studies presented in this thesis was conducted at GROW-School for Oncology and Developmental Biology, Department of Internal Medicine, Division of Hematology, and Department of Transplantation Immunoloy, Tissue Typing Laboratory, at Maastricht University Medical Center+.

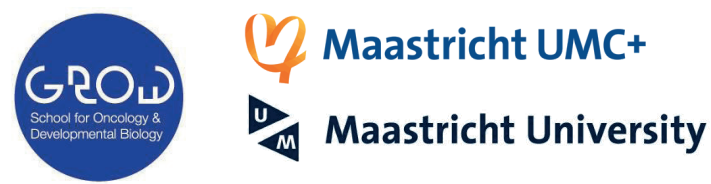




\section{Refining natural killer cell-based immunotherapy}

Strategies to unleash the killer in a suppressive tumor microenvironment

\section{DISSERTATION}

to obtain the degree of Doctor at the Maastricht University, on the authority of the Rector Magnificus,

Prof. dr. Rianne M. Letschert

in accordance with the decision of the Board of Deans,

to be defended in public

on Wednesday, 19 December 2018 at 10 o'clock

by

Niken Miranti Mahaweni

Born on 20 November 1986 in Jakarta, Indonesia 


\section{Supervisors:}

Prof. dr. Gerard M. J. Bos

Prof. dr. Marcel G. J. Tilanus

\section{Co-supervisor:}

Dr. Lotte Wieten

\section{Assessment committee:}

Prof. dr. Dirk De Ruysscher (Chairman) (MAASTRO Clinic, Maastricht University Medical Center+)

Prof. dr. Vivianne C. G. Tjan-Heijnen (Maastricht University Medical Center+)

Dr. Kasper Rouschop (Dept. of Radiotherapy - Maastro Lab, Maastricht University Medical Center+)

Prof. dr. Irma Joosten (Radboud University Medical Center, Nijmegen)

Prof. dr. Tuna Mutis (VU Medisch Centrum/Amsterdam UMC, Amsterdam) 


\section{Contents}

$\begin{array}{lll}\text { Chapter } 1 & \text { General introduction } & 7\end{array}$

\begin{tabular}{ll}
\hline Chapter 2 & Daratumumab augments alloreactive natural killer \\
& cell cytotoxicity towards CD38+ multiple myeloma \\
& cell lines in a biochemical context mimicking tumour \\
& microenvironment conditions
\end{tabular}

Chapter 3 A comprehensive overview of FCGR3A gene variability

by full-length gene sequencing including the identification of $\mathrm{V} 158 \mathrm{~F}$ polymorphism

Chapter 4 Clinical and immunological significance of HLA-E in stem cell transplantation and cancer

Chapter 5 NKG2A expression is not per se detrimental for the anti-multiple myeloma activity of activated natural killer cells in an in vitro system mimicking the tumor microenvironment

\begin{tabular}{|c|c|c|}
\hline Chapter 6 & $\begin{array}{l}\text { Tuning NK cell anti-myeloma reactivity by targeting } \\
\text { inhibitory signaling via HLA class I and HLA-E }\end{array}$ & 123 \\
\hline Chapter 7 & $\begin{array}{l}\text { Less is more: a low glucose concentration during short } \\
\text { and long term cultures is correlated with a better } \\
\text { antitumor response and viability of activated NK cells }\end{array}$ & 157 \\
\hline Chapter 8 & General discussion & 177 \\
\hline \multirow[t]{3}{*}{ Chapter 9} & Summary & 183 \\
\hline & English & \\
\hline & Nederlands & \\
\hline \multirow[t]{4}{*}{ Chapter 10} & Valorisation & 190 \\
\hline & List of publications & 200 \\
\hline & Curriculum vitae & 202 \\
\hline & Acknowledgement & 204 \\
\hline
\end{tabular}


6

| Chapter 1 


\section{General Introduction}




\section{INTRODUCTION}

Our body is protected against a diversity of pathogenic offenses by the exquisitely specialized cells of the immune system that originated from the pluripotent hematopoietic stem cells in the bone marrow (BM). These stem cells could differentiate into common lymphoid progenitor cells or common myeloid progenitor cells [1]. The lymphocytes, comprising B cell, T cell, NK cell, and NK-T cells, originate from the common lymphoid progenitor. The granulocytes -comprising basophils, eosinophils, neutrophils- mast cells, macrophages, and monocytes, were originating from the common myeloid progenitor. The development of lymphocytes mostly takes place in the bone marrow (and the liver during fetal period) for the B cells and the thymus for the T cells [2]. NK cell development, however, is rather unique as it takes place in both BM and lymph node [3]. During each stage of lymphocyte development, precursor cells develop into more differentiated or specialized cells expressing antigen-specific receptors, namely immunoglobulins for B cells, T-cell receptors for T cells, and NK cell receptors for NK cells. All these receptors mainly have functions in differentiating selfmolecules and non-self molecules

\section{MULTIPLE MYELOMA}

Multiple myeloma ( $M M)$ is a malignant disease of plasma cells residing in the BM [4]. Plasma cells are terminally differentiated B cells, which in the normal situation, produce antibodies with different structures and functions and a high affinity for specific antigens [5]. In MM, the plasma cells undergo abnormal clonal expansion and produce an abnormal amount of monoclonal M protein, an abnormal immunoglobulin fragment, as a result of an excess abnormal monoclonal proliferation of plasma cells. The disease has been described as a multifactorial disease, with risk factors including genetic, occupational, clinical, and lifestyle factors [6]. The biological mechanism for the malignant transformation from plasma cells to MM has been described to be a multistep process involving genetic (and epigenetic) aberrations and changes in the BM microenvironment [7].

\section{MM biology, pathogenesis}

To differentiate from an immature B cell to a mature plasma cell, capable of producing antibody, a B cell needs to undergo several genetic alterations namely isotype class switching and somatic hypermutation in the germinal center within the lymph nodes or spleen [8]. These processes involve DNA strand breaks [4, 5], which are highly prone to mutation. In MM, mutations, such as translocation involving the IGH gene encoding the heavy chain of antibody, indeed frequently occur at the immunoglobulin switch region on chromosome 14 (q32.33) $[4,7,8]$. Other mutations affecting a set of partner genes 
such as MM SET, FGFR3, cyclins D1 and D3, MAF are also frequently observed $[5,7,8]$. Mutations acquired during isotype class switching and somatic hypermutation stage are considered to be the primary or first oncogenic event of the multiple oncogenic steps in MM pathogenesis.

After the maturation process, some plasma cells relocate and reside in the BM to become a long -ived plasma cell. It is postulated that the secondary or later oncogenic events of MM oncogenesis take place in the BM because the so-called late-onset translocations as well as gene mutations involving MYC, NRAS and KRAS, FGFR3, p53, $C D K N 2 A / 2 C$ are frequently found in MM and rarely in the monoclonal gammopathy of undetermined clinical significance (MGUS) [7], a premalignant condition which often precedes the development of MM.

In addition to genetic instabilities, the bone marrow microenvironment where MM resides play an important role in the pathogenesis of the disease. BM is physiologically a hypoxic region to support the normal hematopoiesis. The BM of MM patients, however, has been reported to be more hypoxic compared to the normal BM marked by the higher expression of hypoxia-inducible factor (HIF)-1a and $-2 a$ (reviewed by [9]). Additionally, a previous study demonstrated that hypoxia plays a role in MM progression by decreasing the adhesion of MM cells to the BM and therefore promoting MM cells dissemination to the circulation [10]. Furthermore, the interplay between MM cells and BM cells or the extracellular matrix proteins have been shown to support tumor growth, survival, trafficking, and drug resistance and will be discussed in more detail later in the introduction $[7,8]$.

\section{MM clinical}

MM accounts for $10 \%$ of all hematological malignancies in the United States in 2017 [5] and around 1100 people per year are diagnosed with MM in the Netherlands according to the Dutch cancer registry.

MM has been described as an age-related disease and the median age at diagnosis is 70 years and $37 \%$ of patients are younger than 65 years or older than 75 years [7]. Clinically, MM manifestation involves extensive lytic bone lesions, hypercalcemia, anemia, production of $\mathrm{M}$-protein (as a result of excessive antibody production), and kidney failure [11]. The therapeutic regimen for MM composed of high dose chemotherapy (alkylators, such as Melphalan, and corticosteroids) followed by a rescue autologous stem cell transplantation (AutoSCT) [12]. More recently, drugs such as proteasome inhibitors (bortezomib, ixazomib, carfilzomib), immunomodulatory drugs (thalidomide, lenalidomide, pomalidomide), and monoclonal antibodies 
(daratumumab, elotuzumab) have been applied in the clinic to improve the clinical outcome of patients with MM [13]. Nonetheless, MM is still an incurable disease. Despite the newer treatment options and the increase in survival rate [14], the median progression-free survival is approximately between 21-43 months and the median overall survival is between 48-72 months [13], with large differences between younger and older patient.

Given these data, the development of a treatment or a combination of treatments to further improve the clinical outcome of MM patients is desirable.

\section{STEM CELL TRANSPLANTATION}

One of the treatment options for patients with MM is a stem cell transplantation [15]. It is a procedure in which healthy stem cells, derived from the BM, peripheral blood, or umbilical cord, are infused to a patient to replace patient's cells. Depending on the source of the stem cells, a stem cell transplantation could be an autologous stem cell transplantation (AutoSCT) or an allogeneic stem cell transplantation (AlloSCT).

To date, when eligible, AutoSCT is considered to be the treatment of choice for patients with MM under 70 years old $[15,16]$. Initially, an induction chemotherapy will be performed to reduce the tumor burden before patients' stem cells are harvested. The procedure is then followed by a conditioning chemotherapy to eliminate cancer cells and the transplantation of the harvested patient's stem cells. The purpose of this transplantation is to replace patient's stem cells which were destroyed after the administration of chemoor radiotherapy to eradicate MM cells. Although AutoSCT could effectively control the disease [16] and improve the median overall survival of MM patients by approximately 12 months [15], it does not cure MM or completely eradicate the malignant cells, possibly because it provides a minimal immunologic effect against MM cells [17].

AlloSCT, on the other hand, might offer the potential cure for MM. The stem cells for an AlloSCT are mostly obtained either from HLA-matched related or HLA-matched unrelated donors. Since it is derived from an allogeneic source, unlike AutoSCT, AlloSCT is tumor-free and it could elicit a graft-versus-tumor (GVT) effect [17]. NK cells and T cells are the two key players in inducing GVT effect in an AlloSCT.

GVT occurs when donor T cells attack the host tumor cells due to the presentation of tumor-associated/specific antigens or recognition of non-self HLA-peptide complexes by antigen presenting cells and tumor cells. Nonetheless, since T cells could recognize both the foreign HLA and the minor histocompatibility antigens, graft-versus-hostdisease (GVHD) could be an undesired side effect of GVT in an AlloSCT $[18,19]$. 
In an HLA-matched AlloSCT, donor T cells present in the allograft could induce a GVHD effect due to the recognition of the minor histocompatibility antigens exclusively expressed in the recipient [20]. Meanwhile in an HLA-mismatched AlloSCT, donor T cells could also promote the development of GVHD by responding to a specific protein expressed by the highly polymorphic HLA molecules on the recipient cells [20]. AlloSCT is still not recommended as a treatment of choice for most MM patients $[15,21]$ because of the high rates of transplant-related mortality (25-50\%) [17], GVHD $[13,18]$, and the disappointing antitumor effect.

More recently, since the availability of HLA-matched donors, both related and unrelated, is limited, AlloSCTs can be carried out using the stem cells from haploidentical stem cell donors, which provide genotypically partially HLA-matched with the patients [22]. Importantly, recent studies on haploidentical stem cell transplantations (HaploSCT) with T cell depletion have shown encouraging results on patients with MM $[23,24]$ where the incidence of GVHD was low and transplantation outcome was improved. Also in our center, we are running a multicenter trial with haploidentical in MM which demonstrated the feasibility of the approach so far. In the HaploSCT setting, NK cells have been shown to be potent mediators of GVT reactivity [25] and a more detailed mechanism on NK cell biology, their potential in HaploSCT and the role in tumor response will be discussed in this thesis introduction

\section{NK CELL THERAPY}

\section{The general concept of (adoptive) NK cell-based therapy}

NK cells were first discovered more than four decades ago. In 1975, when Kiessling published an article on a lymphocyte population in mice that displayed cytotoxicity against tumor cells without prior stimulation, the term "natural killer cell" was coined to this lymphocyte subset $[26,27]$. However, the development of NK cellbased immunotherapy came into the limelight as a potential cell-based cancer immunotherapy in 2002, when Ruggeri et al demonstrated that NK cells could induce a graft versus leukemia effect in patients with acute myeloid leukemia receiving HaploSCT without inducing GVHD [25]. In a 4T1 breast cancer mice model, our group showed that the depletion of NK cells from an HaploSCT graft in mice receiving HaploSCT resulted in a significantly reduced anti-tumor effect of the transplant [28]. In line with the observation by the Ruggeri group, our result demonstrated that NK cells from haploidentical donors were the key players responsible for the GVT effect and therefore could potentially eliminate both hematological and solid tumors. 
Given the antitumor potency of NK cells, attempts have been made to isolate NK cells and exploit NK cells for adoptive cell therapy, both in the autologous or allogeneic settings (Fig. 1.1). Multiple studies provided proof of principle that adoptive NK cell transfer could be a promising treatment for patients with cancer. Earlier clinical trials in patients having either lymphoma or breast cancer using autologous adoptive NK cell therapy demonstrated that, despite its excellent safety results, autologous NK cells did not exert antitumor responses [29]. In an allogeneic setting, infusion of a high number of allogeneic NK cells into patients having melanoma, renal cell carcinoma, or acute myeloid lymphoma has been shown to be feasible and without significant side effects [30]. Additionally, in the same study, 5 of 19 patients with acute myeloid lymphoma achieved a complete remission. In a more recent serie of studies, adoptive transfer of NK cells has been shown to induce remission and improve disease-free survival in a small number of patients with hematological malignancies [31-35]. In solid tumors, however, the efficacy of adoptive transfer of NK cells against solid tumors is still limited.

Despite the encouraging results of adoptive NK cell therapy from the past and ongoing clinical studies, further investigations for the strategies to improve the clinical efficacy of NK cell therapy are warranted [36]. In light of this unmet need, we aimed, in the current thesis, to investigate potential strategies to enhance the NK cell-antitumor capacity for adoptive NK cell therapy.

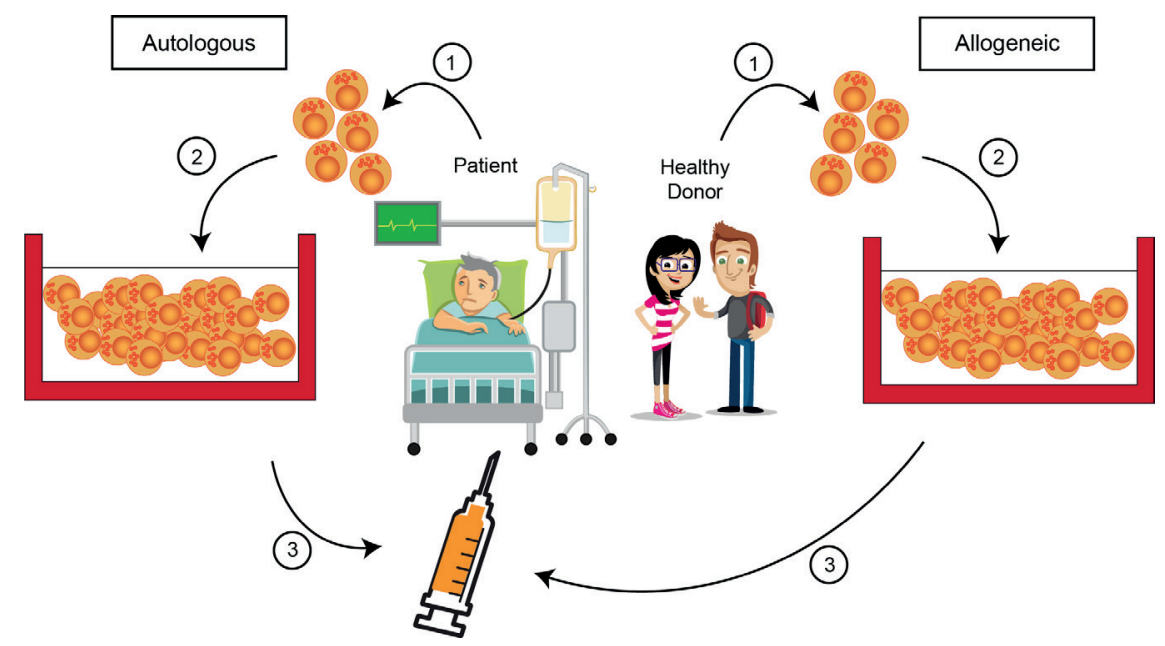

Figure 1.1. Concept of adoptive NK cell transfer. 1) NK cells are isolated from the peripheral blood of either a patient (in autologous setting) or a healthy donor (in allogeneic setting). 2) NK cells are then cultured ex vivo for expansion and activation. 3) The expanded NK cell product is infused to the patient. (Some illustrations in the figure are adapted from www.pngtree.com) 


\section{Potential strength: NK cell function}

When NK cells were first discovered, they were described as lymphocytes with "natural killer" capacity based on the observation that they could kill tumor cells without prior stimulation. Later on, it was revealed that NK cells belonged to a separate subset of lymphocytes that also had other effector functions such as production of cytokines (such as IFN-y, TNF-a, GM-CSF, IL-10) [37-39] and chemokines (such as CCL1, CCL3, CCL4, CCL5) [40-42]. Through these different functions, NK cells are very important for the clearance of certain viral-, parasitic-, and intracellular bacterial infections [43]. Human NK cells make up between $5-15 \%$ of total lymphocytes in the peripheral blood. They can be identified by their extracellular expression of CD56 and the lack of CD3. Classically, human NK cells are grouped into two major subsets based on their levels of CD56 expression, namely CD56 ${ }^{\mathrm{dim}}$ and CD56 bright $\mathrm{NK}$ cells. Approximately $90 \%$ of NK cells found in the blood are CD56 dim and only roughly $10 \%$ are CD56 bright. The majority of CD56 bright NK cells are shown to reside in secondary lymphoid tissues [44] or other tissues such as liver and uterus $[45,46]$.

Functionally, upon activation, CD56 $6^{\mathrm{dim}}$ NK cells have been described to have a more cytotoxic capacity than cytokine-producing capacity. CD56 bright NK cells, on the other hand, are more capable to produce abundant amounts of cytokines and chemokines than killing a target cell [47]. Nonetheless, although CD56 ${ }^{\text {bright }}$ NK cells are the more efficient cytokines producer, CD56 ${ }^{\text {dim }}$ NK cells could significantly contribute to the production of cytokine because they form a significantly greater fraction of the total NK cells [48].

\section{NK cell recognition of a target cell}

One distinctive feature of an NK cell is that it can selectively kill diseased cells -e.g. virally-infected or transformed cells- without damaging healthy normal cells. NK cell could distinguish normal and diseased cells by the expression of different inhibitory and activating molecules on the cell surface of a normal or a diseased cell. Engagement of an inhibitory molecule on a potential target cell to an inhibitory receptor expressed on an NK cell provides an inhibitory signal for the NK cell while a binding of an activating molecule on a potential target cell to an activating receptor on an NK cell provides an activating signal. The sum of the strength of signals transduced determines the extent of an NK cell response.

Based on this concept, there are four types of NK cell recognition and the possible outcomes (Fig. 1.2) [49]: 1) "Healthy cell tolerance" - a normal healthy cell expresses a class I human leukocyte antigen (HLA) which could be recognized by a corresponding inhibitory killer immunoglobulin-like receptor (iKIR) expressed on an NK cell, which 
provides an inhibitory signal. However, some normal healthy cells lowly express HLA (e.g. neural cells) or do not express HLA-class (e.g. erythrocytes) and are normally not killed by an NK cell. This is because normal healthy cells do not produce sufficient activating signals, or alternatively, this type of cells expresses other inhibitory molecules which bind to other inhibitory receptors besides the iKIRs. 2) "Missing self" recognition - a virally-infected cell or a tumor cell, often downregulates HLA-class I molecules, to escape CD8 T-cell lysis, therefore lowering the activation threshold of NK cells and allowing NK cells to be activated in the presence of sufficient activating molecules. 3) "Induced-self" recognition - some virally-infected cells or tumor cells could maintain their HLA-class I expression while at the same time upregulating stressed-induced activating molecules, making them vulnerable for NK cell mediated cytotoxicity.

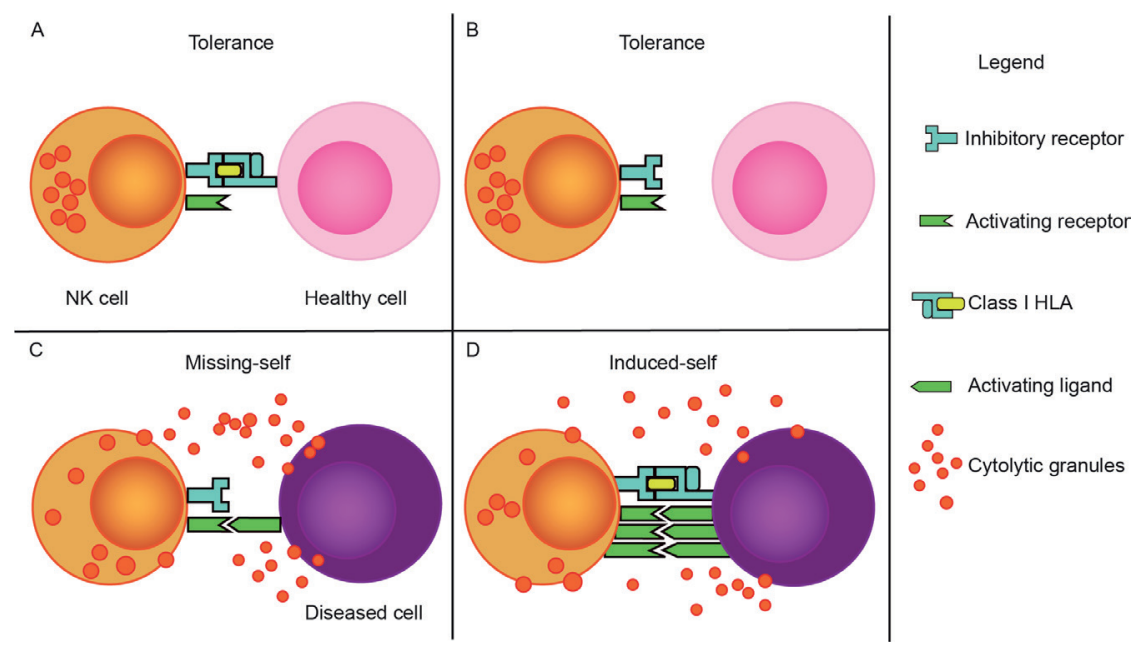

Figure 1.2. Different types of NK cell recognition. Tolerance: NK cell is tolerant to a healthy cell that expresses class I HLA molecule (A) or does not express class I HLA molecule (B) if the healthy cell does not express an activating ligand. Missing-self recognition: when a tumor cell/ infected cell downregulates the class I HLA molecule and expresses an activating ligand, the NK cell would release its cytolytic granules or starts producing cytokines to kill the tumor/infected cell (C). Induced-self recognition: when a tumor cell/infected cell expresses class I HLA molecules and activating ligands, the signal is determined by the strength of inhibitory or activating signals. When the activation signals override the inhibitory signal, NK cells would kill the diseased cell (D). (Figure is adapted from [49]) 


\section{NK cell-mediated cytotoxicity}

Upon recognition of a target cell and reception of activating signals, an NK cell could become activated and initiate the cytotoxic process. There are two distinct mechanisms or pathways known to date for NK cell-mediated cytotoxicity [50]. The first pathway is the granule exocytosis pathway, involving the release of the vesicular contents of cytotoxic granules protein such as perforin, granzymes, and granulysin into the intercellular space [51]. Within the vesicle, cytotoxic granules bind to the LAMP-1 (CD107a) protein on the vesicular membrane. Upon the release of the contents, these vesicular proteins are exposed, enabling the measurement of CD107a protein as a surrogate marker of NK cell activation. Of note, granzyme uptake into a target cell does not require perforin's role to create transmembrane pores as initially proposed [50]. Rather, granzyme uptake is mediated by a receptor-mediated endocytosis, while perforin has been proposed to play a role in disrupting endosomal trafficking after granzyme uptake into the target cell [50]. The second pathway is via the interaction between death ligands of TNF-family members (FasL and TRAIL) expressed on NK cells and their receptors (Fas and TRAIL-R DR4, DR5) expressed on the target cells [52]. FasL and TRAIL are crucial mediators of the caspasedependent target cell apoptosis.

\section{NK cell receptors}

\section{Activating receptors}

As previously mentioned in this thesis, an NK cell response is dictated by the net signals received by the receptors. NK cells express a vast array of both activating and inhibitory receptors. The major activating receptors for NK cells that play a significant role in NK cell cytotoxicity against tumor cells are the family of natural cytotoxicity receptors (NCRs), NKG2D, and DNAM1. Activating receptors on NK cells normally bind to the stress-induced molecules, which are normally absent or lowly expressed by healthy cells but upregulated by a diseased cell as a response to cellular stress, due to malignant transformation or viral infection. The NCR family comprises NKp30, $-44,-46$, and -80 and can recognize virally-associated proteins like BAT-3, HSPG, B7-H6, viral hemagglutinin [53]. NKG2D binds to self-antigens such as MICA/B molecules and UL16-binding proteins (ULBP). The expression of these two ligands is commonly induced by stress [54] or the DNA damage response pathway caused by malignant transformation or heat shock response pathway [53]. DNAM1 belongs to the family of nectin-binding adhesion molecules which bind to nectin proteins such as CD112 (nectin-2) and CD155 (poliovirus receptor -PVR-) [55]. The engagement between DNAM1 and its ligands induces actin polymerization and activation of other surface receptors, allowing a stable interaction between an NK cell and the target cell. Importantly, the upregulation of DNAM1 ligand, CD155, in MM has been reported to result in increasing MM sensitivity to NK cell mediated cytotoxicity [56]. Furthermore, since MM cells express ligands for NKG2D, the binding of NKG2D to either 
MICA/B or UL16-binding protein families express on an MM cell has been shown to an enhanced MM cell killing by NK cells [57-59]

Another essential activating receptor is the CD16a receptor, also known as FcyRllla receptor, which binds to the Fc part of an immunoglobulin $\mathrm{G}(\mathrm{IgG})$ antibody. CD16a is a very potent receptor that does not require a co-activation from other receptors to activate NK cell cytotoxicity and cytokine release [60]. The ligation of CD16a receptor triggers target cell killing via a mechanism called antibody-dependent cell-mediated cytotoxicity (ADCC) [61]. ADCC is the process where CD16 on the NK cell is binding to the Fc part of an antibody bound to a potential target cell leading to potent activation of the NK cell and killing of the target cell. There are several pathways involved in ADCC: 1) secretion of cytotoxic granules, 2) death receptor signaling, and 3) the release of pro-inflammatory cytokines such as IFNy [62]. Although CD16a is a strong inducer of cytotoxicity, several polymorphisms have been found that influence the binding affinity of CD16a and the Fc part of an immunoglobulin. The most studied polymorphism is the V158F polymorphism where a single nucleotide polymorphism (SNP) at the nucleotide position 559 in the cDNA resulted in two allotypes of the receptor, one with low-affinity binding and one with high-affinity binding $[63,64]$. Several clinical studies on several types of malignancies demonstrated that patients having high-binding affinity receptor showed a better outcome (progression-free survival) as compared to patients having low-binding affinity receptor upon monoclonal antibody therapy [65-69].

\section{Inhibitory receptors}

The major inhibitory receptors expressed on an NK cell interact with self-antigens such as the classical and the non-classical class I HLA (HLA A-B-C and HLA-E). KIRs, as briefly touched upon earlier in this thesis, are one of the major inhibitory receptors. Of note, not all KIRs are inhibitory and in addition, some inhibitory and activating KIRs (aKIRs) recognize the same ligands. However, most iKIRs have a stronger binding affinity to the same ligands than their activating counterparts [70]. The KIR family is encoded by 14 highly polymorphic genes (2DL1-2DL5, 3DL1-3DL3, 2DS1-2DS5, and 3DS1) [71]. A KIR comprises two (KIR2D) or three (KIR3D) extra-cellular C2-type Ig-like domains with a long cytoplasmic tail (L) containing immunoreceptor tyrosine-based inhibition motifs (iTIMs) for iKIR or a short cytoplasmic tail (S) containing immunoreceptor tyrosine-based activation motifs (iTAMs) for aKIR [72]. 


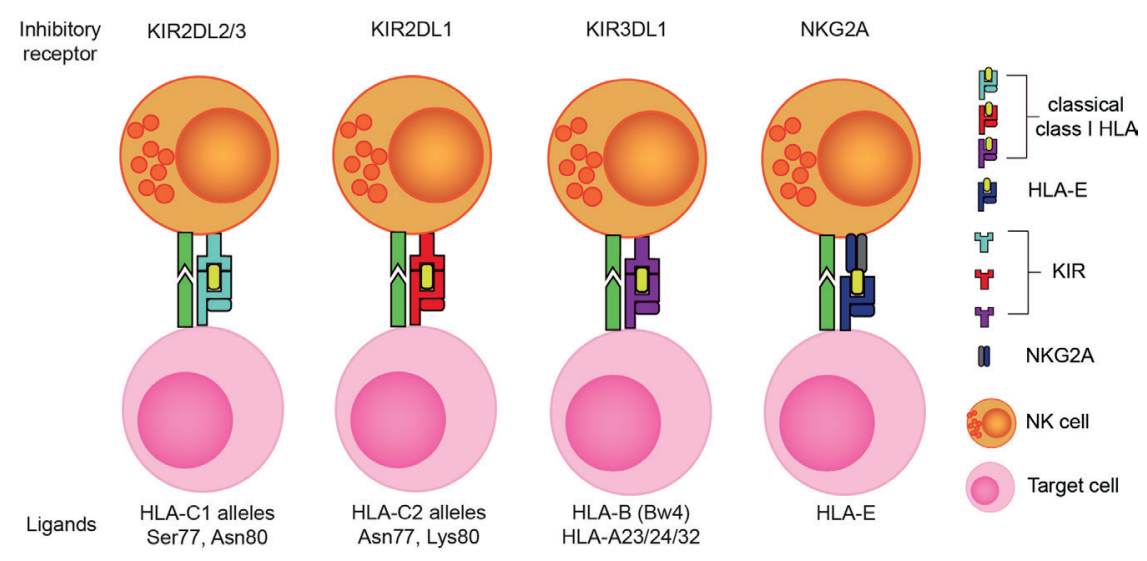

Figure 1.3. KIRs and their ligands.

An iKIR could recognize one group of class I HLA alleles based on the allotypic presence of specific epitopes (Fig. 1.3). KIR2DL1 (CD158a) recognizes group 2 HLA-C alleles characterized by a lysine (Lys) residue at position 80 while KIR2DL2 and KIR2DL3 (CD158b1/-2) recognize group 1 HLA-C alleles characterized by an asparagine (Asn) residue at position 80 [71]. KIR3DL1 (CD158e1) recognizes HLA-B allotypes with Bw4 motifs at positions 77-83, except for $B^{*} 13: 01$ and $B * 13: 02$ and some HLA-A alleles, namely $A^{*} 23: 01, A^{*} 24: 02$, and $A^{*} 32: 01$ [73]. KIR3DL2 (CD158k) is the receptor for HLA-A*03/A*11 [71].

In addition to recognition of classical class I HLA molecules, NK cells express CD94/ NKG2 receptor complex which can recognize non-classical class I HLA molecules (HLA-E). Similar to KIRs, the NKG2 receptor could be an activating (NKG2C) or an inhibitory (NKG2A) receptor. However, the binding affinity of NKG2A to HLA-E has been shown to be 6 folds stronger than the binding of NKG2C to HLA-E [74]. In contrast to the classical class I HLA molecules, HLA-E is less polymorphic with only 15 alleles known to date (http://www.ebi.ac.uk/ipd/imgt/hla) and only 2 of them are functionally important, namely HLA-E*01:01 and HLA-E*01:03[75-77]. Similar to the classical class I HLA molecules, HLA-E is also expressed by virtually all cells in the body. However, HLA-E expression can be upregulated upon an environmental insult or as an escape mechanism of a tumor cell.

\section{NK cell alloreactivity}

Similar to T cells, NK cells distinguish self and non-self antigens by recognizing the HLA molecules expressed on the membrane of a cell. Unlike T cells which are activated by the interaction of the T cell receptor with a peptide-HLA complex displayed by 
antigen-presenting cells or diseased cells, NK cells interaction involving class I HLA molecule-specific inhibitory receptor inhibits NK cell activation [78]. During the development, NK cells are educated to tolerate the self-antigens through a process termed "licensing" wherein NK cells expressing a specific inhibitory receptor specific for an HLA (Figure 1.3) would interact with the cognate HLA and become matured or licensed [79]. Therefore only receptors that have engaged with their cognate HLA during the developmental stage can become responsive upon encountering a target cell at a later stage [53].

KIR genes are randomly expressed and the distribution of KIR differs per NK cells. In addition, the expression of KIRs is independent of HLA expression since KIR genes are located in the chromosome 19 while HLA genes are located in the chromosome 6 Because of the independent expression of KIRs and HLAs, some KIRs might not encounter its cognate HLA during development and therefore are not licensed [80]. NK cells expressing unlicensed KIRs are demonstrated to be hyporesponsive against a target cell that does not express HLA molecules [79].

The "missing self" concept as mentioned previously has been proposed to be the underlying mechanism of NK cell anti-tumor effect in the HSCT in AML patients [81]. When licensed NK cells from the donor express KIRs for which the HLA ligand is missing in the patient cell resulting in a KIR-ligand mismatch, these NK cells are called alloreactive [82]. An ideal alloreactive NK cell donor should, therefore, express one or more HLA epitopes that are absent in the patient and expresses the specific $\mathrm{KIR}$ that can interact with the missing HLA-epitope to create a KIR-ligand mismatch and potentially trigger NK cell activation upon patient's cells that are missing the HLA. A clinical study on MM patients receiving reduced chemotherapy dose and T-cell depleted AlloSCT from unrelated donors showed that showed that KIR-ligand mismatch status between a donor and a patient was protective for relapse [83]. This study highlighted the possible role of NK cell alloreactivity in MM. In line with this study, another clinical study investigating the impact of KIR-ligand mismatched status on the clinical outcome in patients with MM showed that infusion of haploidentical KIR-ligand mismatched NK cells has a positive impact on patient's survival after an AutoSCT [84]. An important issue pointed out by Shi et al was that the number of alloreactive NK cells should be sufficient to obtain an adequate anti-MM effect.

\section{Potential problem: TME and NK cell inhibition}

Tumor cells have attained characteristics to ensure their growth, survival, and progression namely by sustaining proliferative capacity, evading growth suppressors, enabling replicative immortality, invasion and metastasis, inducing angiogenesis, 
resisting apoptosis, deregulating cellular metabolism, escaping immune surveillance, promoting inflammation, and creating mutation as well as genome instability [85]. With these acquired features, tumor cells could shape and alter both cellular and non-cellular components of the tumor niche to support the tumorigenesis [86]. Together these components form the tumor microenvironment (TME). The interaction between tumor cells with the other non-malignant cells, such as fibroblasts, osteoblasts, endothelial cells, as well as immune cells present in the TME happens via the secretion of cytokines, chemokines, growth factors [87]. The result of this interaction could be that the nonmalignant cells become more tumor-promoting cells. Other factors that might be present in the TME such as an elevated level of lactate and hypoxia could be a consequence of tumor cells metabolic activity and progression [88].

In the case of MM, the localization of tumor cells within the BM takes place via the interaction of cell-surface adhesion molecules such as LFA-1 and VLA-4 [89] with their ligands expressed on BM stromal cells and extracellular matrix proteins. In addition to the localization of tumor cells in the BM, this interaction can result in the secretion of IL- 6 by BM stromal cells, thereby supporting MM growth [90].

Although BM is considered physiologically hypoxic, MM cells are thought to be chronically exposed to lower oxygen levels. As reviewed by Hu et al, several studies, showed that in the BM of MM mouse models there was a gradient of hypoxic area correlated with high levels of hypoxia-inducible factor (HIF)-1a [9]. In humans, HIF-1a and HIF-2a were also seen positive in the histopathology specimen of MM patients [9]. More recently, an increased IL-32 expression by MM cells has been associated with a hypoxic signature in patients [91]. The chronic exposure of MM cells to hypoxic microenvironment has been demonstrated to induce the production of VEGF by both MM cells and stromal cells and promote angiogenesis, allowing the expansion of MM cells [92].

As mentioned earlier, lactate is often present in the TME as a metabolic consequence of tumor growth, as a consequence of their increased metabolic needs [93]. Although exact lactate levels in the BM of MM patients are unknown to this date, an increase in lactate serum concentration [94] or lactate dehydrogenase (LDH) [95] has been found in patients with a more severe or aggressive form of MM. A study on MM cell lines and MM BM stromal cells demonstrated that MM cells and stromal cells produced high levels of lactate, as a result of aerobic glycolysis [96]. Additionally, they showed that lactate was reutilized by MM cells as source of energy. However, high levels of lactate have been shown in previous studies to reduce NK cell antitumor response [97, 98]. 
Studies have demonstrated that the TME of MM can be suppressive for NK cell anti-MM activity (reviewed in [99]). Our group has previously demonstrated that hypoxia could diminish NK cell cytotoxicity against MM cell lines [100]. Moreover, MM cells as well as regulatory $T$ cells and myeloid derived suppressor cells, which are abundant in MM patients, produce TGF-ß and can independently suppress NK cells killing capacity [101] and CD16a mediated IFNy production and ADCC [102]. Additionally, MM cells express COX-2 which leads to the production of PGE2 [103] and can inhibit the cytolytic activity of NK cells by suppressing the NK cell response to IL-12 and IL-15 [104, 105].

A study by Benson et al showed that NK cells from MM patients expressed programmed death-1 (PD-1) receptor, a co-signaling molecule acting as an immune checkpoint, and not in healthy donors [106] implying that its ligand (PD-L1) might be expressed on MM cells. Indeed, another study showed that PD-L1 was upregulated on the MM cells and myeloma-propagating pre-plasma cells in the BM of patients [107]. The interaction between PD-1 and PD-L1 could inhibit NK cell cytotoxicity could result in dysfunctional NK cells with decreased cytolytic and cytokine production capacity [108].

In summary, all these factors present in the TME potentially challenge the efficacy of cancer therapies. Therefore it is extremely important to understand how the TME influences the therapy and to study the strategies to bypass this.

\section{Outline of The Thesis}

Although results from current clinical studies on NK cell-based immunotherapy on different types of malignancies are encouraging, the full potential of this therapy has not yet been achieved giving a plenty of opportunities for refinement. Some of the key aspects include: to obtain sufficient numbers of GMP-grade NK cells to infuse into cancer patients; upon infusion or reconstitution after SCT the NK cells should be able to home to the tumor and to survive at the tumor site and NK cells should be capable of mediating their anti-tumor function by killing tumor cells and production of anti-tumor cytokines. Since several TME factors have been shown to hamper NK cell antitumor response, TME will be the focus in this thesis as we anticipate that a suppressive TME is one of the greatest challenges for an NK cell-based therapy. 

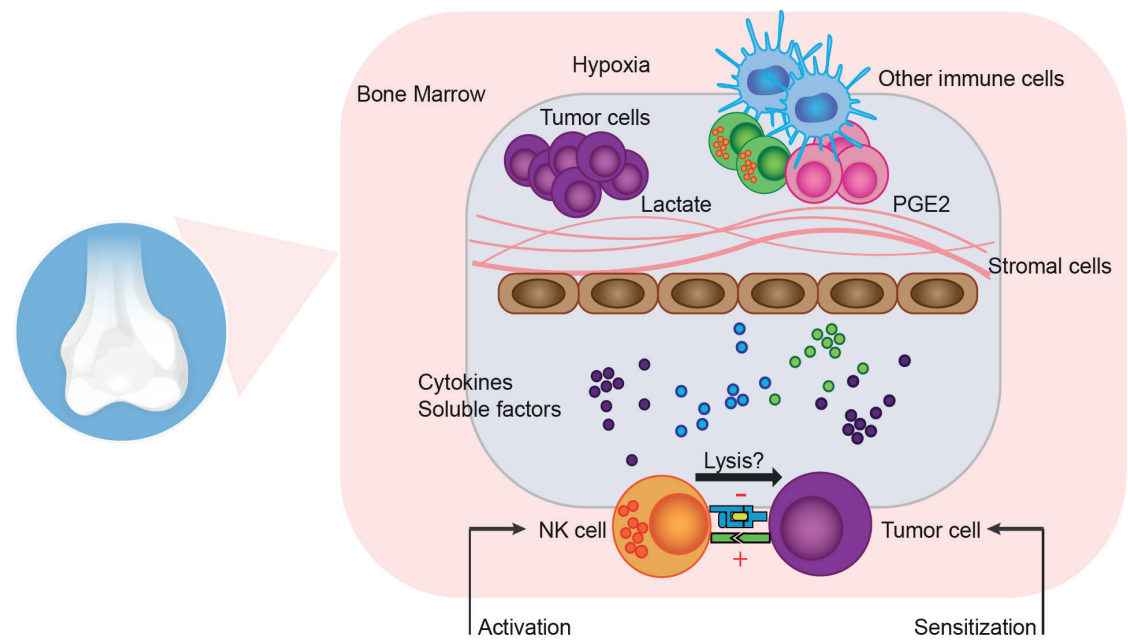

Figure 1.4. Bone marrow tumor microenvironment. The TME of MM is composed of cellular (immune cells, tumor cells, stromal cells, endothelial cells) and non-cellular compartment (hypoxia, cytokines, soluble factors, metabolites). Each of these factors has been described to hamper NK cell activity against MM cells. Strategies to overcome the suppressive effect of TME should include boosting NK cell activation, blocking NK cell inhibition, and tumor cell sensitization. (Some illustrations in the figure are adapted from www.pngtree.com)

We envision that a combination of strategies to, on the one hand, maximize NK cell activation while on the other hand minimizing NK cell inhibition would be a potent way to boost the power of NK cells (Fig. 1.4). We previously showed that activation of NK cells with IL-2 could overcome the inhibitory effect of hypoxia on NK cells [100]. Additionally, we showed that KIR-ligand mismatched NK cells were the better effector cells compared to KIR-ligand matched NK cells [109]. This provided proof of concept for our approach. In the current thesis, we followed up on these findings by investigating several additional strategies to potentiate NK cells in the TME.

In chapter 2, we investigated the influence of a more complex TME by testing whether additional TME factors, such as lactate or PGE2, on top of hypoxia, could inhibit IL2-activated alloreactive NK cells. In this chapter, we also explored the efficacy of a combination strategy of IL-2 activated alloreactive NK cells with an ADCC-triggering antibody, Daratumumab, and the additional relevance of KIR-ligand mismatch in the presence of TME factors.

CD16a (FcyRIlla) is the crucial receptor in NK cell-mediated ADCC. Several polymorphisms, such as V158F polymorphism and L48R/H polymorphism, have been identified to functionally affect the patient's clinical outcome to antibody 
therapy. However, a summary of other polymorphisms present within the gene is not available to date. In chapter 3, we, therefore, aimed to provide an extensive overview of polymorphisms present within the CD16a (FCGR3A) gene by using the $1 \mathrm{KG}$ project database. Additionally, we developed two gene-sequencing methods for a fulllength gene identification of CD16a polymorphisms that will enable future functional studies to unravel the functional consequences of these new polymorphisms.

Our group intends to develop ex vivo-expanded NK cells as a therapy to treat cancer patients. Nonetheless, after an expansion protocol, we and others observed that the majority of NK cells were NKG2A+ cells which have been described to have an inhibitory response upon binding with HLA-E molecules on target cells. In chapter 4, we revisited the role of non-classical class I HLA-E in stem cell transplantation and cancer in a review to give an update on the current knowledge of HLA-E in these two fields. In chapter 5, we subsequently aimed to investigate the relevance of HLA-E NKG2A inhibitory interaction on the antitumor response of activated NK cells in the presence of different biochemical factors to mimic in vivo TME. Here, we also used patient-derived MM cells to better predict for the in vivo response in MM patients. By studying this, we would gain insight into whether the expression of NKG2A on an activated NK cell is a troublesome issue we need to tackle. In chapter $\mathbf{6}$, we discuss the relevance of KIR and NKG2A for NK cell anti-MM response and the strategies to maximize the clinical efficacy of allogeneic NK cell-based therapy to treat MM patients.

In addition to high levels of lactate as a result of tumor aerobic glycolysis and acting as a suppressive factor for NK cells in the TME, the TME of many tumors is characterized by low glucose levels. Whether this is also the case in MM and whether low glucose levels have an effect on the NK cell anti-MM response is not known so far. In chapter 7, we, therefore, aimed to investigate the relevance of a low glucose concentration on the antitumor activity and viability of activated NK cells in short- and long-term cultures. To get an idea of glucose concentrations in vivo, we took samples from MM patients and healthy donors and we tested the effect of glucose on activated NK cells based on these references. By studying this, we would gain insight into the NK cell response towards glucose level and whether we could interfere with glucose concentrations during culture or activation of NK cells to create more potent NK cells.

In chapter 8, we summarized all the observations and findings of this thesis in a general discussion outlining a future perspective for the refinement of NK cell-based immunotherapy. 


\section{REFERENCES}

1. Chaplin DD (2010) Overview of the immune response. J Allergy Clin Immunol 125:S3-S23. doi: 10.1016/j.jaci.2009.12.980

2. Charles A Janeway, Jr, Paul Travers, Mark Walport and MJS (2001) Generation of lymphocytes in bone marrow and thymus. Immunobiol. Immune Syst. Heal. Dis.

3. Farag SS, Caligiuri MA (2006) Human natural killer cell development and biology. Blood Rev 20:123-137. doi: 10.1016/j.blre.2005.10.001

4. Sirohi B, Powles R (2004) Multiple myeloma. Lancet (London, England) 363:875-87. doi: 10.1016/ S0140-6736(04)15736-X

5. Kumar SK, Rajkumar V, Kyle RA, et al (2017) Multiple myeloma. Nat Rev Dis Prim 3:1-20. doi: 10.1038/nrdp.2017.46

6. Sergentanis TN, Zagouri F, Tsilimidos G, et al (2015) Risk Factors for Multiple Myeloma: A Systematic Review of Meta-Analyses. Clin Lymphoma, Myeloma Leuk 15:563-577e3. doi: 10.1016/j.clml.2015.06.003

7. Palumbo A, Anderson K (2011) Multiple myeloma. N Engl J Med 364:1046-1060. doi: 10.1056/ NEJMra1011442

8. Bianchi G, Munshi NC (2015) Pathogenesis beyond the cancer clone(s) in multiple myeloma. Blood 125:3049-3058. doi: 10.1182/blood-2014-11-568881

9. Hu J, Van Valckenborgh E, Menu E, et al (2012) Understanding the hypoxic niche of multiple myeloma: therapeutic implications and contributions of mouse models. Dis Model Mech 5:763771. doi: 10.1242/dmm.008961

10. Azab AK, Hu J, Quang P, et al (2011) Hypoxia promotes dissemination of multiple myeloma through acquisition of Endothelial to Mesenchymal Transition (EMT) features. Blood Conf 53rd Annu Meet Am Soc Hematol ASH 118:5782-5795. doi: 10.1182/blood-2011-09-380410.There

11. Blade J, Cibeira MT, Fernandez de Larrea C, Rosinol L (2010) Multiple myeloma. Ann Oncol 21:vii313-vii319. doi: 10.1093/annonc/mdq363

12. San-Miguel JF, Mateos M-V, Kyle R, et al (2011) Can multiple myeloma become a curable disease? Haematologica 96:1246-8. doi: 10.3324/haematol.2011.051169

13. Rajkumar SV, Kumar S (2016) Multiple Myeloma: Diagnosis and Treatment. Mayo Clin Proc 91:101-119. doi: 10.1016/j.mayocp.2015.11.007

14. Fonseca R, Abouzaid S, Bonafede M, et al (2017) Trends in overall survival and costs of multiple myeloma, 2000-2014. Leukemia 31:1915-1921. doi: 10.1038/leu.2016.380

15. Rajkumar SV (2016) Multiple myeloma: 2016 update on diagnosis, risk-stratification, and management. Am J Hematol 91:719-734. doi: 10.1002/ajh.24402

16. Kumar S (2009) Stem cell transplantation for multiple myeloma. Curr Opin Oncol 21:162-170. doi: 10.1097/CCO.0b013e328324bc04

17. Bensinger WI (2007) Is there still a role for allogeneic stem-cell transplantation in multiple myeloma? Best Pract Res Clin Haematol 20:783-795. doi: 10.1016/j.beha.2007.09.007

18. Gahrton G, Björkstrand B (2008) Allogeneic transplantation in multiple myeloma. Haematologica 93:1295-1300. doi: 10.3324/haematol.13555

19. Hambach L, Spierings E, Goulmy E (2007) Risk assessment in haematopoietic stem cell transplantation: Minor histocompatibility antigens. Best Pract Res Clin Haematol 20:171-187. doi: 10.1016/j.beha.2006.09.002

20. Ringdén $\mathrm{O}$, Karlsson $\mathrm{H}$, Olsson $\mathrm{R}$, et al (2009) The allogeneic graft-versus-cancer effect. Br J Haematol 147:614-633. doi: 10.1111/j.1365-2141.2009.07886.x

21. Dhakal B, Vesole DH, Hari PN (2016) Allogeneic stem cell transplantation for multiple myeloma: is there a future? Bone Marrow Transplant 51:492-500. doi: 10.1038/bmt.2015.325 
22. Spitzer TR (2005) Haploidentical stem cell transplantation: the always present but overlooked donor. Hematol Am Soc Hematol Educ Progr 390-395. doi: 10.1182/asheducation-2005.1.390

23. Chen Y, Lu J, Xu L-P, et al (2018) Safety and efficacy of haploidentical stem cell transplantation for multiple myeloma. Bone Marrow Transplant 10-13. doi: 10.1038/s41409-017-0069-1

24. Castagna L, Mussetti A, Devillier R, et al (2017) Haploidentical Allogeneic Hematopoietic Cell Transplantation for Multiple Myeloma Using Post-Transplantation Cyclophosphamide Graftversus-Host Disease Prophylaxis. Biol Blood Marrow Transplant 23:1549-1554. doi: 10.1016/j. bbmt.2017.05.006

25. Ruggeri L, Capanni M, Urbani E, et al (2002) Effectiveness of Donor Natural Killer Cell Alloreactivity in Mismatched Hematopoietic Transplants. Science (80- ) 295:2097-2100. doi: 10.1126/science. 1068440

26. Kiessling R, Klein E, Wigzell H (1975) "Natural” killer cells in the mouse. Eur J Immunol 112-117. doi: $10.1002 /$ eji.1830050208

27. Kiessling R, Klein E, Pross H, Wigzell H (1975) "Natural” killer cells in the mouse. II. Cytotoxic cells with specificity for mouse Moloney leukemia cells. Characteristics of the killer cell. Eur J Immunol 5:117-121. doi: 10.1002/eji.1830050209

28. Frings PWH, Van Elssen CHMJ, Wieten L, et al (2011) Elimination of the chemotherapy resistant subpopulation of 4T1 mouse breast cancer by haploidentical NK cells cures the vast majority of mice. Breast Cancer Res Treat 130:773-781. doi: 10.1007/s10549-011-1355-z

29. Burns LJ, Weisdorf DJ, DeFor TE, et al (2003) IL-2-based immunotherapy after authologous transplantation for lymphoma and breast cancer induces immune activation and cytokine release: A phase I/II trial. Bone Marrow Transplant 32:177-186. doi: 10.1038/sj.bmt.1704086

30. Miller JS, Soignier Y, Panoskaltsis-mortari A, et al (2005) Successful adoptive transfer and in vivo expansion of human haploidentical NK cells in patients with cancer. Cancer 105:3051-3057. doi: 10.1182/blood-2004-07-2974.Supported

31. Björklund AT, Carlsten M, Sohlberg E, et al (2018) Complete Remission with Reduction of Highrisk Clones following Haploidentical NK Cell Therapy against MDS and AML. Clin Cancer Res clincanres.3196.2017. doi: 10.1158/1078-0432.CCR-17-3196

32. Curti A, Ruggeri L, Addio AD, et al (2011) Successful transfer of alloreactive haploidentical KIR ligand-mismatched natural killer cells after infusion in elderly high risk acute myeloid leukemia patients Successful transfer of alloreactive haploidentical KIR ligand-mismatched natural killer cells. Blood 118:3273-9. doi: 10.1182/blood-2011-01-329508

33. Rubnitz JE, Inaba H, Ribeiro RC, et al (2010) NKAML: A pilot study to determine the safety and feasibility of haploidentical natural killer cell transplantation in childhood acute myeloid leukemia. J Clin Oncol 28:955-959. doi: 10.1200/JCO.2009.24.4590

34. Bachanova V, Linda J. Burns DHM (2010) Allogeneic Natural Killer Cells for Refractory. Cancer Immunol Immunother 59:1739-1744. doi: 10.1007/s00262-010-0896-Z.

35. Ciurea SO, Schafer JR, Bassett R, et al (2017) Phase 1 clinical trial using mbIL21 ex vivo expanded donor-derived NK cells after haploidentical transplantation. Blood 130:1857-1869. doi: 10.1182/blood-2017-05-785659.

36. Guillerey C, Huntington ND, Smyth MJ (2016) Targeting natural killer cells in cancer immunotherapy. Nat Immunol 17:1025-1036. doi: 10.1038/ni.3518

37. Trinchieri G (1989) Biology of Natural Killer Cells. Adv Imunol 47:187-376. doi: 10.1016/S00652776(08)60664-1

38. De Sanctis JB (1997) Secretion of cytokines by natural killer cells primed with interleukin-2 and stimulated with different lipoproteins. Immunology 90:526-533. doi: 10.1046/j.13652567.1997.00174.x

39. Vivier E, Raulet DH, Moretta A, et al (2011) Innate or Adaptive Immunity? The Example of Natural Killer Cells. Science (80- ) 331:44-49. doi: 10.1126/science.1198687 
40. Nieto M, Navarro F, Perez-Villar JJ, et al (1998) Roles of chemokines and receptor polarization in NK-target cell interactions. J Immunol 161:3330-3339.

41. Robertson MJ (2002) Role of chemokines in the biology of natural killer cells. J Leukoc Biol 71:173-83. doi: 10.1189/JLB.71.2.173

42. Fauriat C, Long EEO, Ljunggren H-G, Bryceson YT (2010) Regulation of human NK-cell cytokine and chemokine production by target cell recognition. Immunobiology 115:2167-2176. doi: 10.1182/blood-2009-08-238469.A

43. Lanier LL (2000) The origin and functions of natural killer cells. Clin Immunol 95:14-18. doi: 10.1006/clim.1999.4816

44. Fehniger T a, Cooper M a, Nuovo GJ, et al (2003) CD56 bright natural killer cells are present in human lymph nodes and are activated by T cell - derived IL-2 : a potential new link between adaptive and innate immunity. Blood 101:3052-3057. doi: 10.1182/blood-2002-09-2876. Supported

45. Poli A, Michel $T$, Thérésine $M$, et al (2009) CD56bright natural killer (NK) cells: an important NK cell subset. Immunology 126:458-65. doi: 10.1111/j.1365-2567.2008.03027.x

46. Michel T, Poli A, Cuapio A, et al (2016) Human CD56 bright NK Cells: An Update. J Immunol 196:2923-2931. doi: 10.4049/jimmunol.1502570

47. Cooper MA, Fehniger TA, Caligiuri MA (2001) The biology of human natural killer-cell subsets. Trends Immunol 22:633-640. doi: 10.1016/S1471-4906(01)02060-9

48. Campbell KS, Hasegawa J (2013) Natural killer cell biology: An update and future directions. J Allergy Clin Immunol 132:536-544. doi: 10.1016/j.jaci.2013.07.006

49. Lanier LL (2005) Nk Cell Recognition. Annu Rev Immunol 23:225-274. doi: 10.1146/annurev. immunol.23.021704.115526

50. Smyth MJ, Cretney E, Kelly JM, et al (2005) Activation of NK cell cytotoxicity. Mol Immunol 42:501-510. doi: 10.1016/j.molimm.2004.07.034

51. Topham NJ, Hewitt EW (2009) Natural killer cell cytotoxicity: how do they pull the trigger? Immunology 128:7-15. doi: 10.1111/j.1365-2567.2009.03123.x

52. Yoon SR, Kim T-D, Choi I (2015) Understanding of molecular mechanisms in natural killer cell therapy. Exp Mol Med 47:e141. doi: 10.1038/emm.2014.114

53. Pegram HJ, Andrews DM, Smyth MJ, et al (2010) Activating and inhibitory receptors of natural killer cells. Immunol Cell Biol 89:216-224. doi: 10.1038/icb.2010.78

54. Long EO (2002) Tumor cell recognition by natural killer cells. Semin Cancer Biol 12:57-61. doi: 10.1006/scbi.2001.0398

55. Konjević G, Vuletić A, Martinović KM, Džodić R (2017) The Role of Activating and Inhibitory NK Cell Receptors in Antitumor Immune Response. Nat Kill Cells. doi: 10.5772/intechopen.69729

56. Morisaki T, Onishi H, Katano M (2012) Cancer immunotherapy using NKG2D and DNAM-1 systems. Anticancer Res 32:2241-2247. doi: 32/6/2241 [pii]

57. Zingoni A, Cecere F, Vulpis E, et al (2015) Genotoxic Stress Induces Senescence-Associated ADAM10-Dependent Release of NKG2D MIC Ligands in Multiple Myeloma Cells. J Immunol 195:736-748. doi: 10.4049/jimmunol.1402643

58. Soriani A, Zingoni A, Cerboni C, et al (2009) ATM-ATR-dependent up-regulation of DNAM-1 and NKG2D ligands on multiple myeloma cells by therapeutic agents results in enhanced NKcell susceptibility and is associated with a senescent phenotype. Blood 113:3503-3511. doi: 10.1182/blood-2008-08-173914

59. Carbone E, Neri P, Mesuraca M, et al (2005) IMMUNOBIOLOGY HLA class I, NKG2D, and natural cytotoxicity receptors regulate multiple myeloma cell recognition by natural killer cells. Blood 105:251-258. doi: 10.1182/blood-2004-04-1422.Supported

60. Bryceson YT, March ME, Ljunggren H-G, Long EO (2006) Synergy among receptors on resting NK cells for the activation of natural cytotoxicity and cytokine secretion. Blood 107:159-166. doi: 10.1182/blood-2005-04-1351 
61. Seidel UJE, Schlegel P, Lang P (2013) Natural Killer Cell Mediated Antibody-Dependent Cellular Cytotoxicity in Tumor Immunotherapy with Therapeutic Antibodies. Front Immunol 4:76. doi: 10.3389/fimmu.2013.00076

62. Wang W, Erbe AK, Hank JA, et al (2015) NK Cell-Mediated Antibody-Dependent Cellular Cytotoxicity in Cancer Immunotherapy. Front Immunol 6:368. doi: 10.3389/fimmu.2015.00368

63. Koene HR, Kleijer M, Algra J, et al (1997) Fc gammaRIlla-158V/F polymorphism influences the binding of IgG by natural killer cell Fc gammaRIIla, independently of the Fc gammaRIIIa-48L/R/H phenotype. Blood 90:1109-14.

64. Wu J, Edberg JC, Redecha PB, et al (1997) A novel polymorphism of FcyRIIla (CD16) alters receptor function and predisposes to autoimmune disease. J Clin Invest 100:1059-1070. doi: $10.1172 / \mathrm{JCl} 119616$

65. Weng W-K, Levy R (2003) Two immunoglobulin G fragment C receptor polymorphisms independently predict response to rituximab in patients with follicular lymphoma. J Clin Oncol 21:3940-7. doi: 10.1200/JCO.2003.05.013

66. Cartron G, Dacheux L, Salles G, et al (2002) Therapeutic activity of humanized anti-CD20 monoclonal antibody and polymorphism in IgG Fc receptor FcgammaRIlla gene. Blood 99:754758. doi: 10.1182/blood.V99.3.754

67. Musolino A, Naldi N, Bortesi B, et al (2008) Immunoglobulin G fragment C receptor polymorphisms and clinical efficacy of trastuzumab-based therapy in patients with HER-2/neupositive metastatic breast cancer. J Clin Oncol 26:1789-96. doi: 10.1200/JCO.2007.14.8957

68. Bibeau F, Lopez-Crapez E, Di Fiore F, et al (2009) Impact of FcyRIla-FcyRIIla Polymorphisms and KRAS Mutations on the Clinical Outcome of Patients With Metastatic Colorectal Cancer Treated With Cetuximab Plus Irinotecan. J Clin Oncol 27:1122-1129. doi: 10.1200/JCO.2008.18.0463

69. Taylor RJ, Chan S-L, Wood A, et al (2009) FcyRIlla polymorphisms and cetuximab induced cytotoxicity in squamous cell carcinoma of the head and neck. Cancer Immunol Immunother 58:997-1006. doi: 10.1007/s00262-008-0613-3

70. Stewart CA, Laugier-Anfossi F, Vely F, et al (2005) Recognition of peptide-MHC class I complexes by activating killer immunoglobulin-like receptors. Proc Natl Acad Sci 102:13224-13229. doi: 10.1073/pnas.0503594102

71. Campbell KS, Purdy AK (2011) Structure/function of human killer cell immunoglobulinlike receptors: Lessons from polymorphisms, evolution, crystal structures and mutations. Immunology 132:315-325. doi: 10.1111/j.1365-2567.2010.03398.x

72. Middleton D, Curran M, Maxwell L (2002) Natural killer cells and their receptors. Transpl Immunol 10:147-164. doi: 10.1016/S0966-3274(02)00062-X

73. Velardi A (2008) Role of KIRs and KIR ligands in hematopoietic transplantation. Curr Opin Immunol 20:581-587. doi: 10.1016/j.coi.2008.07.004

74. Kaiser BK, Barahmand-pour F, Paulsene W, et al (2005) Interactions between NKG2x Immunoreceptors and HLA-E Ligands Display Overlapping Affinities and Thermodynamics. J Immunol 174:2878-2884. doi: 10.4049/jimmunol.174.5.2878

75. Joosten SA, Sullivan LC, Ottenhoff THM (2016) Characteristics of HLA-E Restricted T-Cell Responses and Their Role in Infectious Diseases. J Immunol Res. doi: 10.1155/2016/2695396

76. Lauterbach N, Wieten L, Popeijus HE, et al (2015) Peptide-induced HLA-E expression in human PBMCs is dependent on peptide sequence and the HLA-E genotype. Tissue Antigens 85:242251. doi: $10.1111 / \tan .12525$

77. Kraemer T, Blasczyk R, Bade-Doeding C, et al (2014) HLA-E: A Novel Player for Histocompatibility. J Immunol Res 2014:1-7. doi: 10.1155/2014/352160

78. Narni-Mancinelli E, Vivier E, Kerdiles YM (2011) The "T-cell-ness" of NK cells: Unexpected similarities between NK cells and T cells. Int Immunol 23:427-431. doi: 10.1093/intimm/dxr035

79. Anfossi N, André P, Guia S, et al (2006) Human NK Cell Education by Inhibitory Receptors for MHC Class I. Immunity 25:331-342. doi: 10.1016/j.immuni.2006.06.013 
80. Orr MT, Lanier LL (2010) Natural Killer Cell Education and Tolerance. Cell 142:847-856. doi: 10.1016/j.cell.2010.08.031

81. Ruggeri L (2002) Effectiveness of Donor Natural Killer Cell Alloreactivity in Mismatched Hematopoietic Transplants. Science (80- ) 295:2097-2100. doi: 10.1126/science.1068440

82. Ruggeri L, Capanni M, Casucci M, et al (1999) Role of Natural Killer Cell Alloreactivity in HLAMismatched Hematopoietic Stem Cell Transplantation. Blood 94:333-339.

83. Kröger N, Shaw B, lacobelli S, et al (2005) Comparison between antithymocyte globulin and alemtuzumab and the possible impact of KIR-ligand mismatch after dose-reduced conditioning and unrelated stem cell transplantation in patients with multiple myeloma. $\mathrm{Br} J$ Haematol 129:631-643. doi: 10.1111/j.1365-2141.2005.05513.x

84. Shi J, Tricot G, Szmania S, et al (2008) Infusion of haplo-identical killer immunoglobulin-like receptor ligand mismatched NK cells for relapsed myeloma in the setting of autologous stem cell transplantation. Br J Haematol 143:641-53. doi: 10.1111/j.1365-2141.2008.07340.x

85. Hanahan D, Weinberg RA (2011) Hallmarks of cancer: the next generation. Cell 144:646-74. doi: 10.1016/j.cell.2011.02.013

86. Wang M, Zhao J, Zhang L, et al (2017) Role of tumor microenvironment in tumorigenesis. J Cancer 8:761-773. doi: 10.7150/jca.17648

87. Balkwill FR, Capasso M, Hagemann T (2012) The tumor microenvironment at a glance. J Cell Sci 125:5591-5596. doi: 10.1242/jcs.116392

88. Lyssiotis CA, Kimmelman AC (2017) Metabolic Interactions in the Tumor Microenvironment. Trends Cell Biol 27:863-875. doi: 10.1016/j.tcb.2017.06.003

89. Lemaire M, Deleu S, De Bruyne E, et al (2011) The Microenvironment and Molecular Biology of the Multiple Myeloma Tumor. Adv Cancer Res. doi: 10.1016/B978-0-12-386469-7.00002-5

90. Vidriales MB, Anderson KC (1996) Adhesion of multiple myeloma cells to the bone marrow microenvironment: implications for future therapeutic strategies. Mol Med Today 2:425-431. doi: 10.1016/1357-4310(96)84846-5

91. Zahoor M, Westhrin M, Aass KR, et al (2017) Hypoxia promotes IL-32 expression in myeloma cells, and high expression is associated with poor survival and bone loss. Blood Adv 1:2656-2666. doi: 10.1182/bloodadvances.2017010801

92. Giuliani N, Storti P, Bolzoni M, et al (2011) Angiogenesis and multiple myeloma. Cancer Microenviron 4:325-337. doi: 10.1007/s12307-011-0072-9

93. Hirschhaeuser F, Sattler UGA, Mueller-Klieser W, et al (2011) Lactate: a metabolic key player in cancer. Cancer Res 71:6921-5. doi: 10.1158/0008-5472.CAN-11-1457

94. Ustun C, Fall P, Szerlip HM, et al (2002) Multiple myeloma associated with lactic acidosis. Leuk Lymphoma 43:2395-2397. doi: 10.1080/1042819021000040116

95. Hatakeyama N, Daibata M, Nemoto Y, et al (2001) Lactate Dehydrogenase Production and Release in a Newly Established Human Myeloma Cell Line. 273:267-273.

96. Fujiwara S, Wada N, Kawano Y, et al (2015) Lactate, a putative survival factor for myeloma cells, is incorporated by myeloma cells through monocarboxylate transporters 1. Exp Hematol Oncol 4:12. doi: 10.1186/s40164-015-0008-z

97. Scott KEN, Cleveland JL (2016) Lactate Wreaks Havoc on Tumor-Infiltrating T and NK Cells. Cell Metab 24:649-650. doi: 10.1016/j.cmet.2016.10.015

98. Husain Z, Huang Y, Seth P, Sukhatme VP (2013) Tumor-Derived Lactate Modifies Antitumor Immune Response: Effect on Myeloid-Derived Suppressor Cells and NK Cells. J Immunol 191:1486-1495. doi: 10.4049/jimmunol.1202702

99. Godfrey J, Benson DM (2012) The role of natural killer cells in immunity against multiple myeloma. Leuk Lymphoma 53:1666-1676. doi: 10.3109/10428194.2012.676175

100. Sarkar S, Germeraad WT V, Rouschop KMA, et al (2013) Hypoxia induced impairment of NK cell cytotoxicity against multiple myeloma can be overcome by IL-2 activation of the NK cells. PLoS One 8:e64835. doi: 10.1371/journal.pone.0064835 
101. Ghiringhelli F, Ménard C, Terme M, et al (2005) CD4 + CD25 + regulatory T cells inhibit natural killer cell functions in a transforming growth factor- $\beta$-dependent manner. J Exp Med 202:10751085. doi: 10.1084/jem.20051511

102. Trotta R, Col JD, Yu J, et al (2008) TGF- Utilizes SMAD3 to Inhibit CD16-Mediated IFN- Production and Antibody-Dependent Cellular Cytotoxicity in Human NK Cells. J Immunol 181:3784-3792. doi: 10.4049/jimmunol.181.6.3784

103. Ladetto M, Vallet S, Trojan A, et al (2005) Cyclooxygenase-2 (COX-2) is frequently expressed in multiple myeloma and is an independent predictor of poor outcome. Blood 105:4784-4791. doi: 10.1182/blood-2004-11-4201.Supported

104. Baginska J, Viry E, Paggetti J, et al (2013) The Critical Role of the Tumor Microenvironment in Shaping Natural Killer Cell-Mediated Anti-Tumor Immunity. Front Immunol 4:490. doi: 10.3389/ fimmu.2013.00490

105. Kalinski P (2012) Regulation of immune responses by prostaglandin E2. J Immunol (Baltimore, Md 1950) 188:21-28. doi: 10.4049/jimmunol.1101029

106. Jr Benson DM, Bakan CE, Mishra A, et al (2010) The PD-1 / PD-L1 axis modulates the natural killer cell versus multiple myeloma effect : a therapeutic target for CT-011, a novel monoclonal anti PD-1 antibody. Blood 116:2286-2294. doi: 10.1182/blood-2010-02-271874.The

107. Yousef S, Marvin J, Steinbach M, et al (2015) Immunomodulatory molecule PD-L1 is expressed on malignant plasma cells and myeloma-propagating pre-plasma cells in the bone marrow of multiple myeloma patients. Blood Cancer J 5:e285. doi: 10.1038/bcj.2015.7

108. Beldi-Ferchiou A, Caillat-Zucman S (2017) Control of NK cell activation by immune checkpoint molecules. Int J Mol Sci. doi: 10.3390/ijms18102129

109. Sarkar S, van Gelder M, Noort W, et al (2015) Optimal selection of natural killer cells to kill myeloma: the role of HLA-E and NKG2A. Cancer Immunol Immunother 64:951-963. doi: $10.1007 / \mathrm{s} 00262-015-1694-4$ 
30 | Chapter 2 


\section{Daratumumab augments alloreactive} natural killer cell cytotoxicity towards CD38+ multiple myeloma cell lines in a biochemical context mimicking tumour microenvironment conditions

Niken M. Mahaweni1,2, Gerard M. J. Bos1, Constantine S. Mitsiades3, Marcel G. J. Tilanus2, Lotte Wieten2

1 Division of Hematology, Department of Internal Medicine, Maastricht University Medical Center+, Maastricht, The Netherlands

2 Department of Transplantation Immunology, Tissue Typing Laboratory, Maastricht University Medical Center+, Maastricht, The Netherlands

3 Department of Medical Oncology, Dana-Farber Cancer Institute and Department of Medicine, Harvard Medical School, Boston, Massachusetts, USA

Cancer Immunol Immunother. 2018 Jun;67(6):861-872. doi: 10.1007/s00262018-2140-1. 


\section{ABSTRACT}

Natural killer (NK) cell-based immunotherapy is a promising novel approach to treat cancer. However, NK cell function has been shown to be potentially diminished by factors common in the tumor microenvironment (TME). In this study, we assessed the synergistic potential of antibody-dependent cell-mediated cytotoxicity (ADCC) and killer immunoglobin-like receptor (KIR)-ligand mismatched NK cells to potentiate NK cell antitumor reactivity in multiple myeloma (MM). Hypoxia, lactate, prostaglandin E2 (PGE2) or combinations were selected to mimic the TME. To investigate this, NK cells from healthy donors were isolated and NK cell ADCC capacity in response to MM cells was assessed in flow cytometry-based cytotoxicity and degranulation (CD107a) assays in the presence of TME factors (TMEFs). Hypoxia, lactate and PGE2 reduced cytotoxicity of NK cells against myeloma target cells. The addition of daratumumab (anti-CD38 antibody) augmented NK cell cytotoxicity against target cells expressing high CD38 but not against CD38 low or negative target cells also in the presence of TME. Co-staining for inhibitory KIRs and NKG2A demonstrated that daratumumab enhanced degranulation of all NK cell subsets. Nevertheless, KIR-ligand mismatched NK cells were slightly better effector cells than KIR-ligand matched NK cells.

In summary, our study shows that combination therapy using strategies to maximize activating NK cell signaling by triggering ADCC in combination with an approach to minimize inhibitory signaling through a selection of KIR-ligand mismatched donors, can help to overcome the NK-suppressive TME. This can serve as a platform to improve the clinical efficacy of NK cells. 
Daratumumab augments alloreactive natural killer cell cytotoxicity towards CD38+ multiple myeloma cell lines in a biochemical context mimicking tumour

microenvironment conditions

$\begin{array}{ll}\text { Abbreviations } \\ \text { ADCC } & \text { Antibody-dependent cell-mediated cytotoxicity } \\ \text { BM } & \text { Bone marrow } \\ \text { CS-BLI } & \text { Compartment specific-bioluminescence imaging } \\ \text { FCS } & \text { Fetal calf serum } \\ \text { HaploSCT } & \text { Haploidentical stem cell transplantation } \\ \text { HLA } & \text { Human leukocyte antigen } \\ \text { IL-2 } & \text { Interleukin 2 } \\ \text { KIR } & \text { Killer immunoglobulin-like receptor } \\ \text { mAbs } & \text { Monoclonal antibodies } \\ \text { MM } & \text { Multiple myeloma } \\ \text { NK cell } & \text { Natural killer cell } \\ \text { PBMC } & \text { Peripheral blood mononuclear cell } \\ \text { PGE2 } & \text { Prostaglandin E2 } \\ \text { TME } & \text { Tumor microenvironment } \\ \text { TMEFs } & \text { Tumor microenvironmental factor }\end{array}$




\section{INTRODUCTION}

NK cell-based immunotherapy is a promising therapeutic approach to treat cancer. NK cells selectively target cancer cells and induce potent anti-cancer responses while sparing non-cancer cells [1]. A potential obstacle to NK cell therapy is the suppressive tumor microenvironment (TME). The TME contributes to the acquisition of therapyresistant cancer cells posing a potential limitation for any anticancer therapy including immunotherapy [2, 3]. TME factors (TMEFs), for example: hypoxia [4, 5]; prostaglandin E2 (PGE2) [6]; lactate [7, 8]; galectin-3 [9]; platelet-derived growth factor [10]; transforming growth factor $\beta 1$ [11]; as well as the presence of other immune cells such as myeloidderived suppressor cells $[12,13]$, have also been shown to contribute to diminished NK antitumor reactivity. Hence, to further optimize the clinical response of adoptive NK cell therapy, clinically applicable strategies to potentiate the NK cell anti-tumor response, which facilitate the NK cell function in the suppressive TME, are warranted.

The activation of NK cells is determined by the signaling balance between inhibitory and activating NK cell receptors. Either maximizing activating signaling or reducing inhibitory signaling would be a feasible strategy to improve NK cell efficacy. Activating receptors, typically bind to stress-induced ligands expressed by diseased or transformed cells. The most potent activating NK cell receptor is CD16, a low-affinity Fc receptor which binds to the Fc portion of an IgG antibody triggering antibody-dependent cellmediated cytotoxicity (ADCC) [14]. The current availability of a large array of clinicalgrade monoclonal antibodies (mAbs) to treat cancer provides a potent opportunity to enhance the NK cell anti-cancer response via the ligation of CD16 to a cancer antigenspecific antibody subsequently resulting in cancer cell death [15]. The ADCC effect of different therapeutic mAbs such as rituximab, obinutuzumab, trastuzumab, and cetuximab has been described to be mainly NK cell-dependent [16]. Nijhof et al [17] also reported that daratumumab, a more recently engineered $m A b$ against CD38, could trigger NK cell ADCC activity against multiple myeloma (MM) cells. Moreover, one study reported that rituximab could trigger ADCC even under $1 \% \mathrm{O}_{2}$ albeit at a lower level than under $20 \% \mathrm{O}_{2}$ [5]. Inhibitory receptors such as killer immunoglobulin-like receptors (KIRs), interact with human leukocyte antigen (HLA) class I molecules, expressed on the membrane of nearly all healthy cells, to prevent autoreactivity. Approaches to minimize signaling via strongly inhibitory NK receptors, such as KIRs and NKG2A, and to reduce the activation threshold for NK cell activation might be especially crucial in situations where there are already many inhibitory signals present. We recently demonstrated that, also under hypoxic conditions, KIR-ligand mismatched NK cells were more potent effector cells against MM than KIR-ligand matched NK cells [18]. 
Daratumumab augments alloreactive natural killer cell cytotoxicity towards CD38+ multiple myeloma cell lines in a biochemical context mimicking tumour

\section{microenvironment conditions}

Inhibitory KIRs and CD16 are both primarily expressed on CD56dim cells and previous studies showed that a KIR-ligand interaction might negatively influence NK cell-mediated ADCC $[16,19,20]$. In this study, we, therefore, hypothesized that the combination of triggering ADCC and KIR-ligand mismatching could provide a potent platform to potentiate the NK cell antitumor response in the TME. To study this hypothesis, we used daratumumab to evaluate the NK cell-mediated ADCC response to MM cells in the presence of a selected combination of TME factors. These selected TME factors hypoxia, lactate and PGE2 are frequently found in the TME of many tumors and have been described to hamper NK cell antitumor response. In addition, we determined whether KIR-ligand mismatched NK cells were more potent than matched NK cells under these conditions. For the experiments, we used IL-2 activated NK cells to resemble the clinical situation where ex vivo (IL-2) activated NK cells will be infused into cancer patients. 


\section{MATERIALS AND METHODS}

\section{Cell lines and culture}

The K562 cell line was cultured in IMDM and 10\% fetal calf serum (FCS). The OPM2, UM-9, RPMI8226/s cell lines were cultured in RPMI1640 and 10\% FCS, the L363 cell line was cultured in RPMI1640 and 15\% FCS, the JJN-3 cell line was cultured in $40 \%$ IMDM and $40 \%$ low glucose DMEM with 20\% FCS. All cell culture media were supplemented with $100 \mathrm{U} / \mathrm{mL}$ penicillin (Gibco) and $100 \mu \mathrm{g} / \mathrm{mL}$ streptomycin (Gibco). K562 and RPMI8226/s were obtained from American Type Culture Collection (ATCC, Rockville, MD, USA). OPM-2, L363, and JJN-3 were obtained from Deutsche Sammlung von Mikroorganismen und Zellkulturen (DSMZ GmbH, Braunschweig, Germany). UM-9 was a gift from Dr. A. Martens, Vrije Universiteit Medisch Centrum (VUMC), The Netherlands. All culture media were from Gibco, Breda, The Netherlands and FCS was produced by Greiner Bio-One International, $\mathrm{GmbH}$. All cell lines were cultured at $37^{\circ}$ $\mathrm{C}$ in humidified air containing $5 \% \mathrm{CO}_{2}$ with $21 \% \mathrm{O}_{2}$ (Sanyo MCO-20AIC, Sanyo Electric Co, Japan).

\section{NK cell isolation and activation}

NK cells were isolated from fresh blood derived from healthy donors after signing informed consent or from healthy donor's HLA-typed buffy coats. Donors with an HLA-C1+C2+Bw4+ genotype were selected. The use of buffy coats, being a byproduct of a required Medical Ethical Review Committee (METC) procedure, does not need ethical approval in the Netherlands under the Dutch Code for Proper Secondary Use of Human Tissue. These buffy coats were anonymous, and the individuals from whom the samples originated did not object to their use. PBMCs were obtained by density gradient centrifugation of the donor sample using lymphoprep (AxisShield). NK cells were subsequently isolated by negative selection with an NK cell isolation kit using MACS beads and columns according to manufacturer's protocol (Miltenyi Biotec, GmbH). For short term activation, NK cells were cultured in RPMI1640 medium (Gibco) supplemented with $10 \%$ fetal calf serum (Greiner Bio-One), 100 $\mathrm{U} / \mathrm{mL}$ penicillin (Gibco) and $100 \mu \mathrm{g} / \mathrm{mL}$ streptomycin (Gibco) at $37^{\circ} \mathrm{C}$ in humidified air containing $5 \% \mathrm{CO}_{2}$ with $21 \% \mathrm{O}_{2}$ (Sanyo MCO-20AIC, Sanyo Electric Co, Japan). NK cells were activated overnight with $1000 \mathrm{IU} / \mathrm{ml}$ recombinant human IL-2 (Proleukin, Novartis).

\section{CD107a degranulation assay}

To assess NK cell degranulation against MM target cells (tumor cells), CD107a expression on NK cells was analyzed using flow cytometry. Target cells were plated in 24 wells plates at a concentration of $2 \times 10^{6}$ cells $/ \mathrm{mL}$ per well and incubated 
Daratumumab augments alloreactive natural killer cell cytotoxicity towards CD38+ multiple myeloma cell lines in a biochemical context mimicking tumour

microenvironment conditions

overnight at $37^{\circ} \mathrm{C}$ in humidified air containing $5 \% \mathrm{CO}_{2}$ with $21 \% \mathrm{O}_{2}$ (Sanyo MCO20AIC, Sanyo Electric Co, Japan) or 0,6\% O (Invivo, 1000 Ruskinn Technology Ltd, Bridgend, UK). Prior to the assay, IL-2 activated NK cells were harvested, washed, and subjected to 1 -h incubation with either $50 \mathrm{mM}$ sodium L-lactate (Sigma), 100 $\mathrm{ng} / \mathrm{mL}$ prostaglandin (Sigma), or medium. Target cells were pre-incubated for 30 min with 1 $\mu \mathrm{g} / \mathrm{mL}$ daratumumab (Genmab) or trastuzumab (Roche) or, as a control, with medium at $21 \% \mathrm{O}_{2}$ (ambient air) or $0,6 \% \mathrm{O}_{2}$ (hypoxia). TMEF-exposed NK cells were then, in duplicate wells, co-cultured with the target cells in 1:1 effector:target ratio and $2 \mu \mathrm{l}$ anti-CD107a-Horizon V450 (H4A3, BD Biosciences) was added per well. After $1 \mathrm{~h}$ of coculture, monensin (BD Biosciences) was added. After another $3 \mathrm{~h}$, the plate was placed on ice to stop the reaction. Cells were then stained on ice with anti-CD3-APC/ H7 (SK7, BD Biosciences), anti-CD56-PeCy7 (B159, BD Biosciences), anti-KIR2DL1-APC (143211, R\&D), anti-KIR2DL2/3/S2-PE (DX27, Miltenyi Biotec), anti-KIR3DL1-FITC (DX9, Miltenyi Biotec) and anti-NKG2A-PC5.5 (Z199, Beckman Coulter).

\section{Analysis of KIR-ligand matched and mismatched NK cells}

Using Luminex-SSO, we determined the genotypic expression of the HLA-class I epitopes of UM9 (C1+C2-Bw4-) and RPMI8226/s (HLA C1+C2+Bw4-) at the genomic level. KIR-ligand matched NK cells for UM9 were KIR2DL2/3+ while for RPMI8226/s they were KIR2DL2/3+, KIR2DL1+, or the combination of KIR2DL2/3+ and KIR2DL1+. KIR-ligand mismatched NK cells for UM9 were KIR2DL1+, KIR3DL1+ or the combination of KIR2DL1+ and KIR3DL1+, while for RPMMI8226/s they were KIR3DL1+.

\section{Cytotoxicity assay}

The NK cell cytotoxicity potential against tumor cells was determined in a 4-h flow cytometry-based assay. Tumor cells were labeled using CellTracker' ${ }^{m} \mathrm{CM}-\mathrm{Dil}$ Dye (Molecular Probes ${ }^{\mathrm{TM}}$, USA) and were incubated overnight at $37^{\circ} \mathrm{C}$ in humidified air containing $5 \% \mathrm{CO}_{2}$ with $21 \% \mathrm{O}_{2}$ (ambient air) (Sanyo MCO-20AIC, Sanyo Electric Co, Japan) or $0,6 \% \mathrm{O}_{2}$ (hypoxia) (Invivo 2,1000 Ruskinn Technology Ltd, Bridgend, UK). Prior to the assay, IL-2 activated NK cells were harvested and washed followed by 1-h incubation with either $50 \mathrm{mM}$ sodium lactate (Sigma) or $100 \mathrm{ng} / \mathrm{mL}$ PGE2(Sigma) or medium. Tumor cells were pre-incubated for $30 \mathrm{~min}$ with $1 \mu \mathrm{g} / \mathrm{mL}$ daratumumab, trastuzumab, rituximab (Roche), or medium under ambient air or hypoxia. After preincubation of with TMEFs, NK cells were co-cultured with labeled tumor cells in 1:1 effector:target ratio for $4 \mathrm{~h}$ in duplicates. After $4 \mathrm{~h}$, dead Dil-labeled tumor cells were measured with Live/Dead ${ }^{\circledR}$ Fixable Aqua Dead Cell Stain Kit (Molecular Probes ${ }^{\mathrm{TM}}$, USA). Specific cytotoxicity was determined by the equation: (\% dead tumor cells - \% spontaneous tumor cells death)/(100 \% - \% spontaneous tumor cells death) x 100 . 


\section{Compartment specific-bioluminescence (CS-BLI) cyototoxicity assay}

The assay was performed as described previously [21]. In short, luciferase positive cell lines RPMI8226/s, JJN3, and L363 were plated in optical 96-well plates (Corning) at 4000 or 6500 cells per well. NK cells, pre-incubated for 30 min with either lactate or PGE2, were added at a 1:5 effector:target ratio. After $24 \mathrm{~h}$ of co-culture, luciferin (Xenogen Corp) was added, and plates were incubated for an additional $30 \mathrm{~min}$ at $37^{\circ} \mathrm{C}$ followed by immediate measurement of the bioluminescence using a Luminoskan (Labsystems). The percentage tumor cell killing was calculated by: $100 \%$ - (CS-BLI signal with NK cells/CS-BLI signal without NK cells) x $100 \%$.

\section{Flow cytometry}

Expression of MM specific antigens such as CD38-PE (BD Biosciences) and the above mentioned NK cell receptors were measured using flow cytometry. Cells were washed with PBS (Gibco) and stained first for dead cells using Live/Dead ${ }^{\circledR}$ Fixable Aqua Dead Cell Stain Kit (Molecular Probes ${ }^{\mathrm{TM}}$, USA) for 30 min on ice in the dark. Cells were further washed with FACS buffer (PBS, 1\% FCS) and stained with antibodies for 30 min on ice in the dark. All flow cytometric analyses were performed with BD FACS Canto II. Data were analyzed with FlowJo 10.1r5 64 bit software.

\section{Statistical analysis}

All statistical analysis was performed with GraphPad Prism V software (Graphpad Software Inc, San Diego, CA, USA) using two-tailed non-parametric $t$ test with repeated measure (Wilcoxon signed rank test). * indicates a $p$ value of $<0.05$. 
Daratumumab augments alloreactive natural killer cell cytotoxicity towards CD38+ multiple myeloma cell lines in a biochemical context mimicking tumour microenvironment conditions 


\section{RESULTS}

The tumor microenvironmental factors lactate and PGE2 can inhibit NK cell cytotoxicity against MM cells.

To study the effect of combinations of TMEFs on NK cell function, we used co-cultures of IL-2 activated primary NK cells with either MM cell lines or the HLA class I deficient K562 line. Previous studies observed that lactate and PGE2 concentrations of up to 40 $\mathrm{mM}$ (lactate) and $50 \mathrm{ng} / \mathrm{ml}$ (PGE2) could be found in tumors [22, 23]. To determine the NK cell potentiating effect of antibodies in a severely suppressive TME, we performed a dose titration (supplementary Fig. 1) and selected $50 \mathrm{mM}$ lactate and $100 \mathrm{ng} / \mathrm{ml}$ PGE2 as concentrations to combine with hypoxia. As expected from our previous study [4], hypoxia $\left(0.6 \% \mathrm{O}_{2}\right)$ alone did not influence cytotoxicity of IL-2 activated NK cells against all cell lines tested when compared to ambient air $\left(21 \% \mathrm{O}_{2}\right)$ conditions (supplementary Fig. 2). However, the combination of hypoxia and lactate reduced NK cell cytotoxicity ranging between a 1.63 fold reduction (for RPMI8226/s) to a 2.61 fold reduction (for OPM-2) (Fig. 1b). The average fold reduction of NK cell cytotoxicity for all cell lines together was 2.28 fold ( $p<0.0001$, Fig. $1 d$ ). The effect of the combination of hypoxia and PGE2 was less profound than the combination of hypoxia and lactate. It did not reduce NK cell cytotoxicity against $\mathrm{K} 562$. For the $\mathrm{MM}$ cell lines, the reduction ranged between 1.23 fold reduction (for UM-9) and 1.58 fold reduction (for JJN-3) (Fig. 1c). The average fold reduction of NK cell cytotoxicity against all cell lines tested was 1.26 ( $p<0.0001$, Fig. $1 d$ ). To exclude the possibility that the inhibition was due to an increase in NK cell death caused by the TMEFs itself, we tested the viability of NK cells which demonstrated no differences in the percentage of dead NK cells in the presence of TMEFs (supplementary Fig. 3). 
microenvironment conditions

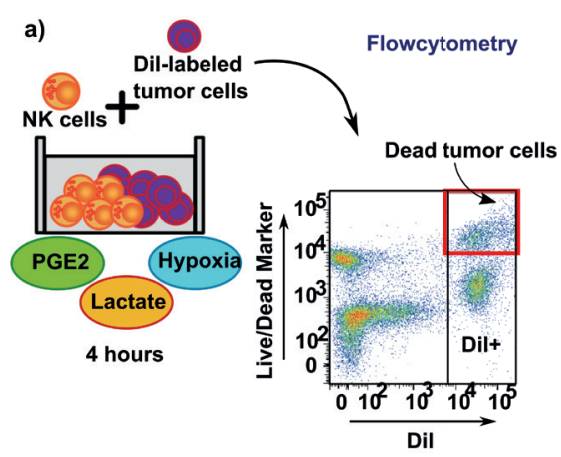

d)
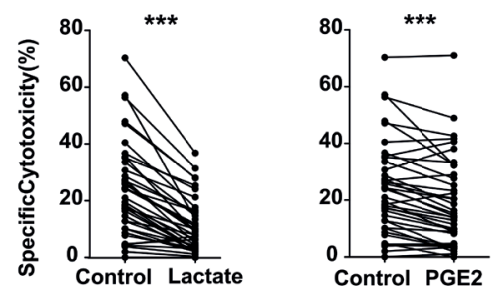

b)
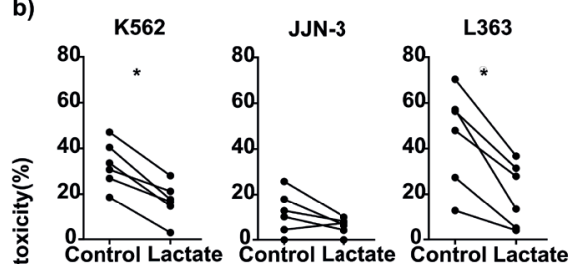

OPM-2
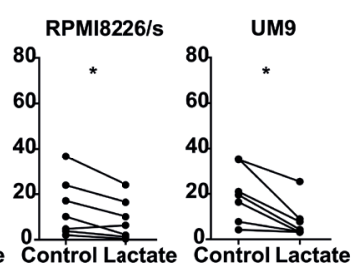
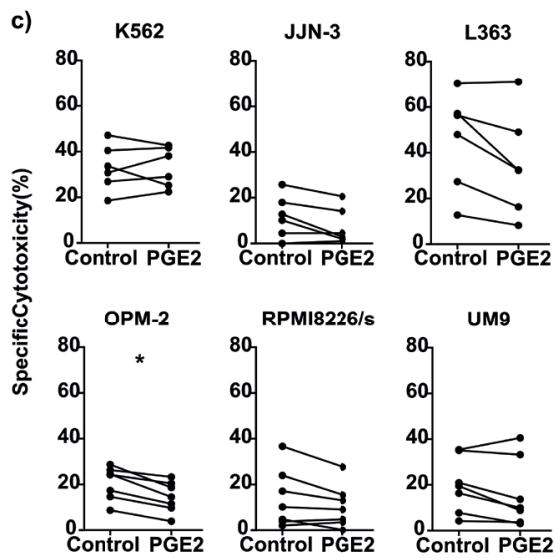

Figure 1. Analysis of the effect of combinations of tumor microenvironmental factors on the antitumor capacity of IL-2 activated NK cells. a Summary of the experimental set up: bloodderived NK cells were activated with IL-2 overnight. The following day, the NK cells were washed and incubated for 1 hour with either PGE2 or lactate followed by a 4 hours cytoxicity assay with Dil-labeled tumor cells that had been overnight incubated under hypoxia $\left(0.6 \% \mathrm{O}_{2}\right)$. b-d Specific cytotoxicity of NK cells against K562, JJN-3, L363, OPM-2, RPMI8226, or UM9 cell lines under hypoxia without (control) or with lactate (b) or PGE2 (c). Data in $b$ and $c$ are from $n=6$ different NK cell donors (every dot represents one donor). $\mathbf{d}$ Data from all cell lines used in $\mathrm{b}$ and $\mathrm{c}$ were pooled and statistical analysis was performed on pooled data. ${ }^{*}=p<0.05,{ }^{* * *}=p<0.0001$. 


\section{Triggering ADCC with Daratumumab can augment NK cell antitumor reactivity in the presence of single or combinations of TMEFs}

To investigate whether ADCC triggering antibodies (daratumumab, trastuzumab, rituximab) could potentiate the NK cell antitumor response in the presence of TMEFs, we performed cytotoxicity assays with or without incubation of the tumor cells with antibodies. In the presence of hypoxia alone, all three antibodies could boost NK cell cytotoxicity when NK cells were co-cultured with cell lines expressing the target antigens (supplementary Fig. 4). We selected daratumumab to further evaluate the ADCC effect in the presence of combinations of TMEFs.

For daratumumab to trigger ADCC, the CD38 antigen expression on the cell surface must persist under TME conditions. We therefore determined CD38 expression on myeloma cells upon culture with TMEFs. Flow cytometry showed that RPMI8226/s and UM9 were high in CD38 expression, while OPM-2 was low in CD38. L363, JJN-3, and K562 were CD38-negative. Moreover, CD38 expression levels remained constant in the presence of TMEF (Fig. 2). In subsequent cytotoxicity assays, we showed that daratumumab enhanced NK cell cytotoxicity against the CD38-high MM cell lines, from $20 \%$ to $45 \%$ for UM9 and from $14 \%$ to $33 \%$ for RPMI8226/s (Fig. 3). Importantly, daratumumab enhanced the NK cell anti-MM response in the presence of all tested combinations of TMEF. For UM9, under hypoxic and lactate conditions, the increase in ADCC was lower compared with the increase in the hypoxia only condition $(p=0.0023)$. Daratumumab did not trigger NK cell-mediated ADCC under any of the conditions where NK cells were co-cultured with CD38low OPM-2 cells, suggesting that the expression level of CD38 on target cells was important for the potential of the antibody to induce NK cell-mediated ADCC. As expected, daratumumab also did not enhance or reduce the killing of the CD38 negative cell lines JJN-3, L363, K562. 

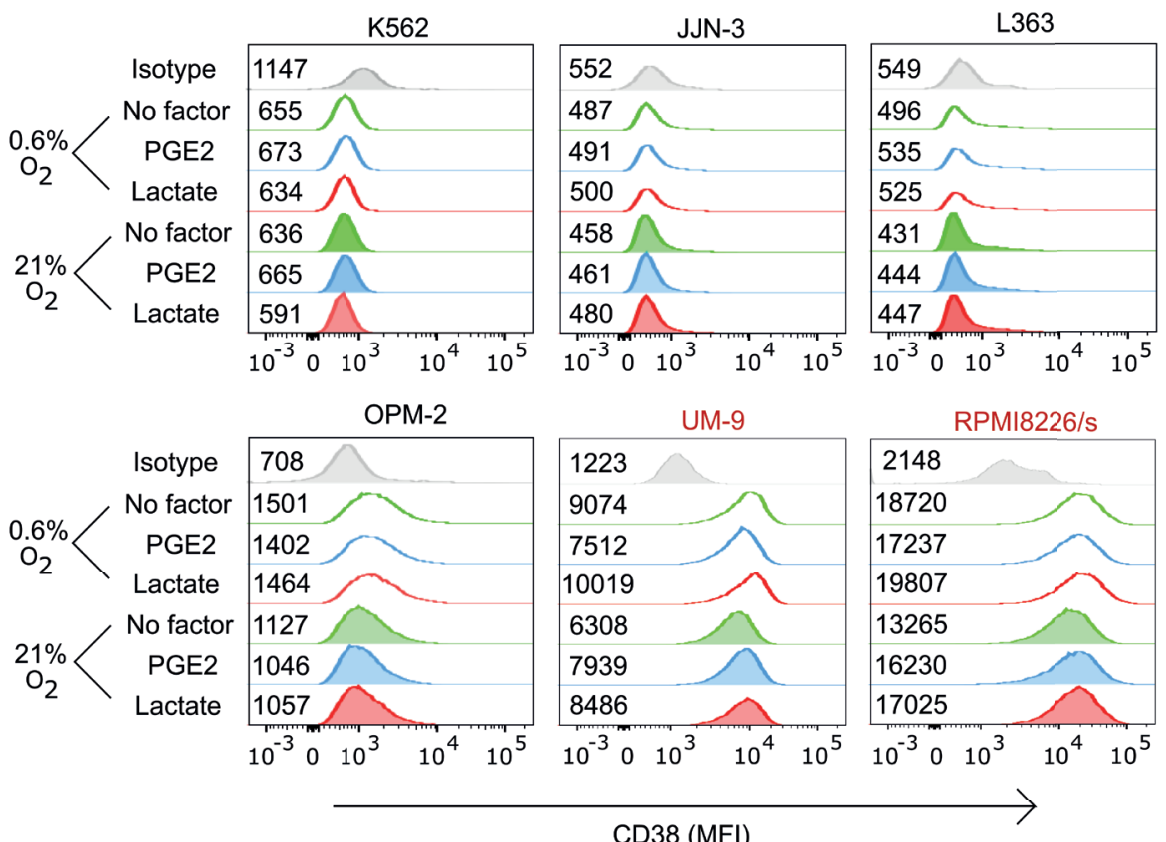

CD38 (MFI)

Figure 2. Analysis of the effect of hypoxia, lactate, PGE2 or combination thereof on CD38 expression levels of multiple myeloma cell lines. UM9, RPMI8226, OPM-2, JJN-3, L363 and K562 cell lines were cultured overnight under $0.6 \%$ or $21 \% \mathrm{O}_{2}$ followed by $4 \mathrm{~h}$ incubation with or without PGE2 or lactate. CD38 expression was determined using flow cytometry. The median fluorescence index $(\mathrm{MFI})$ is indicated next to each histogram. Figure is representative of 3 independent experiments. Cell lines having high CD38 expression are denoted in red. 


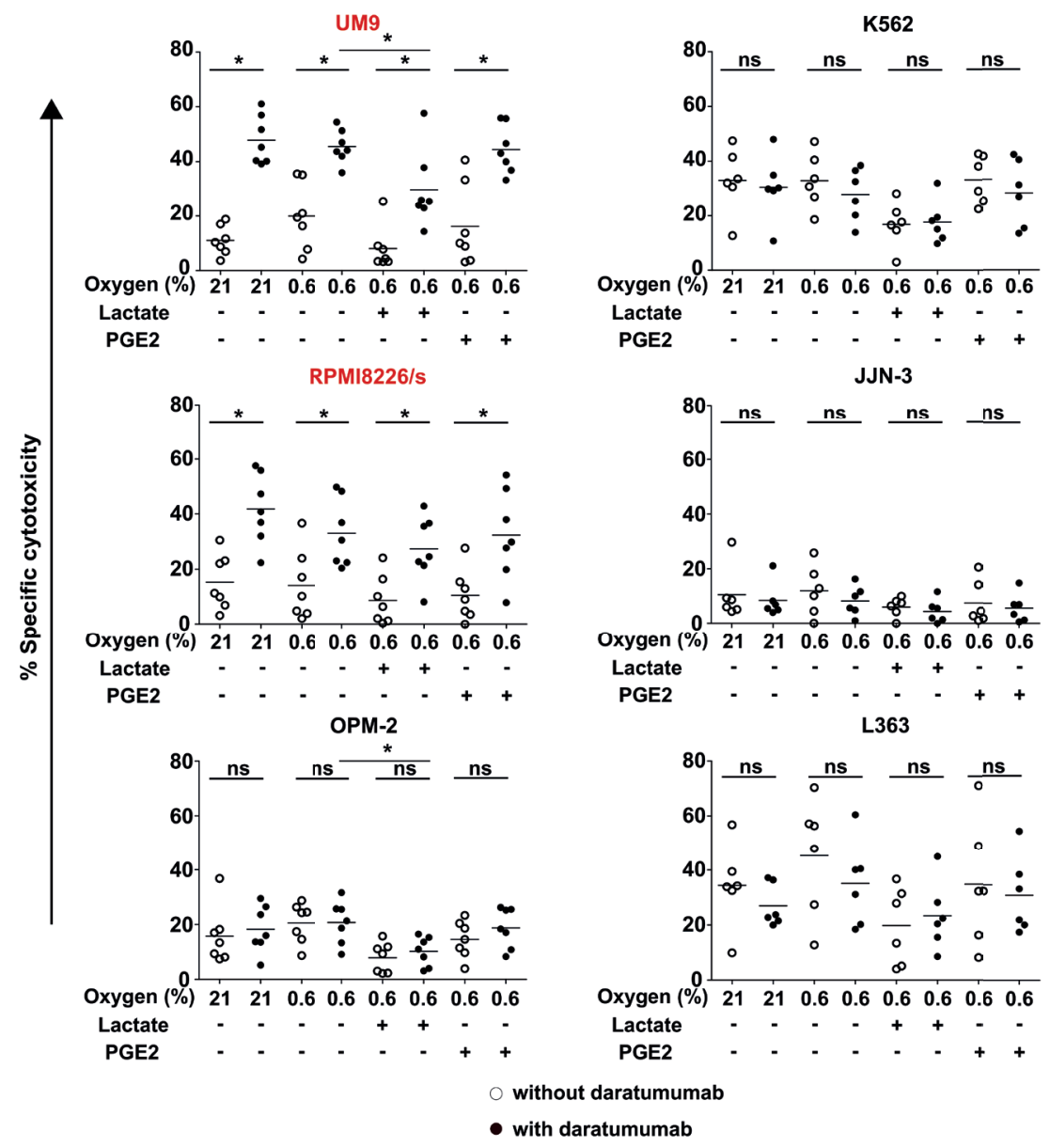

Figure 3. Effect of daratumumab on NK cell killing of CD38-high cells under suppressive TMEF. Dil-labeled UM9, RPMI8226, OPM-2, K562, JJN-3 and L363 were incubated overnight at $0.6 \%$ or $21 \%$ $\mathrm{O}_{2}$. The next day, tumor cells were pre-incubated with daratumumab while overnight IL-2 activated NK cells were pre-incubated with PGE2 or lactate followed by co-culture in a cytotoxicity assay. Graphs show specific cytotoxicty data. White dots = without daratumumab, black dots = with daratumumab. ${ }^{*}=\mathrm{p}<0.05, \mathrm{n}=6$ independent donors (K562, JJN-3, and L363) or 7 donors (UM9, RPMI8226, OPM2). Cell lines having high CD38 expression are denoted in red. 
Daratumumab augments alloreactive natural killer cell cytotoxicity towards CD38+ multiple myeloma cell lines in a biochemical context mimicking tumour

microenvironment conditions

\section{Daratumumab induces NK cell death}

NK cells also express low levels of CD38 which potentially binds daratumumab and they could therefore be a target of daratumumab-mediated killing. Since reducing the number of effector cells could be detrimental, we also evaluated whether there was an increase in the number of dead NK cells after addition of daratumumab. In the absence of daratumumab and tumor cells, the average percentage of dead NK cells after $4 \mathrm{~h}$ of culture was $21.49 \%$ compared with $36.66 \%$ in the presence of daratumumab (Fig. 4a). This phenomenon was also observed in the presence of TMEF. An increase in the percentage of dead NK cells in conditions with daratumumab was also observed after $4 \mathrm{~h}$ co-culture with the different tumor cell as well as in the presence of TMEF lines ( $p<0.0001$, Fig. 4b). An increased NK cell death by daratumumab was observed after only $2 \mathrm{~h}$ and cell death further increased to $60 \%$ after $24 \mathrm{~h}$ (Fig. 5a). Induction of NK cell death was not observed with trastuzumab which binds Her2/neu that is not present on NK cells. Furthermore, the effect of daratumumab was comparable for conditions with $0.1,1$ and $10 \mathrm{ug} / \mathrm{ml}$ of antibody. We also observed a higher percentage of CD107a positive NK cells in all conditions with daratumumab. This demonstrates that NK cells increasingly degranulate upon addition of daratumumab suggesting that they get activated by- and mediate ADCC against- other NK cells with daratumumab bound to their surface (Fig. 5b). 
a)

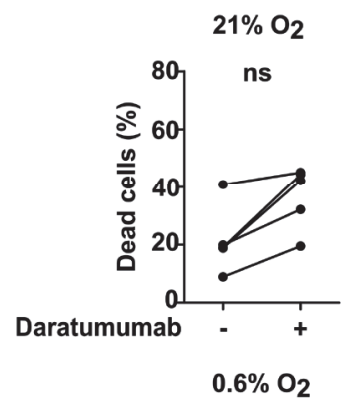

$21 \% \mathrm{O}_{2}+$ Lactate

$21 \% \mathrm{O}_{2}+\mathrm{PGE} 2$
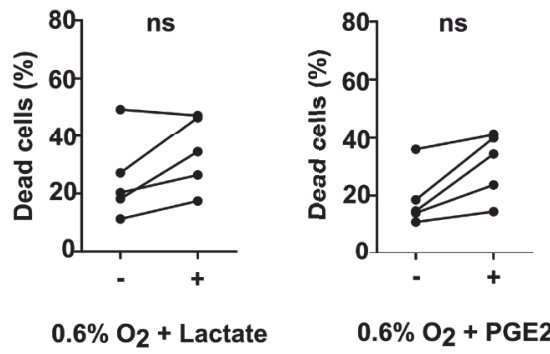

$0.6 \% \mathrm{O}_{2}+\mathrm{PGE} 2$
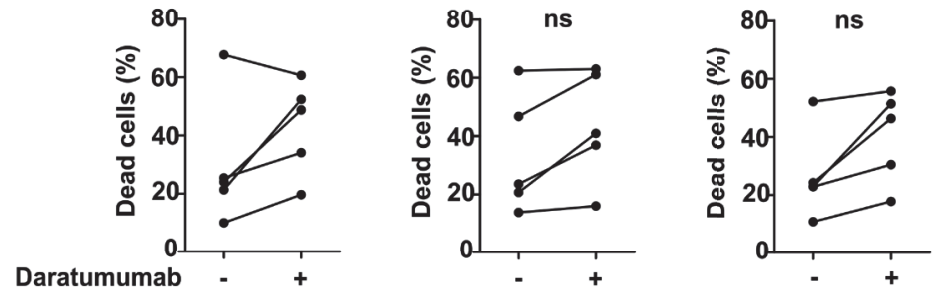

b)
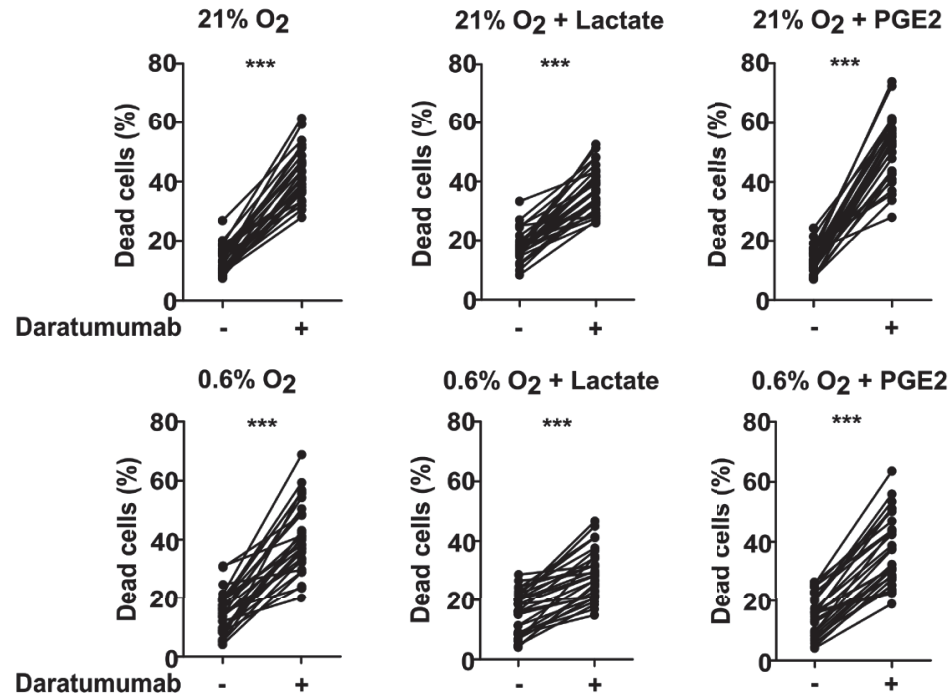

$$
0.6 \% \mathrm{O}_{2}+\mathrm{PGE} 2
$$
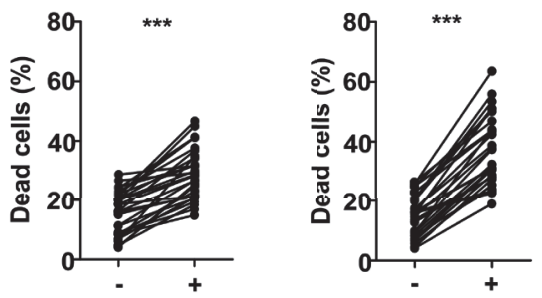

Figure 4. NK cell death in the absence or presence of daratumumab. a IL-2 activated NK cells were incubated with daratumumab. After $4 \mathrm{~h}$, NK cell death was determined by flow cytometric analysis of a life-death marker. $\mathrm{n}=5$ independent donors, every dot indicates one donor. $\mathbf{b}$ Incubation of IL-2 activated NK cells with daratumumab and tumor cell lines (K562, JJN-3, L363, OPM-2, UM9 or RPMI8226/s), $n=5$ independent donors, every dot indicates one donor. For graphs in $\mathbf{b}$, NK cells from each of the five donors were incubated with all six cell lines. Data from all cell lines were collectively plotted in one graph. ${ }^{* * *}=p<0.0001$ 
Daratumumab augments alloreactive natural killer cell cytotoxicity towards CD38+ multiple myeloma cell lines in a biochemical context mimicking tumour

microenvironment conditions
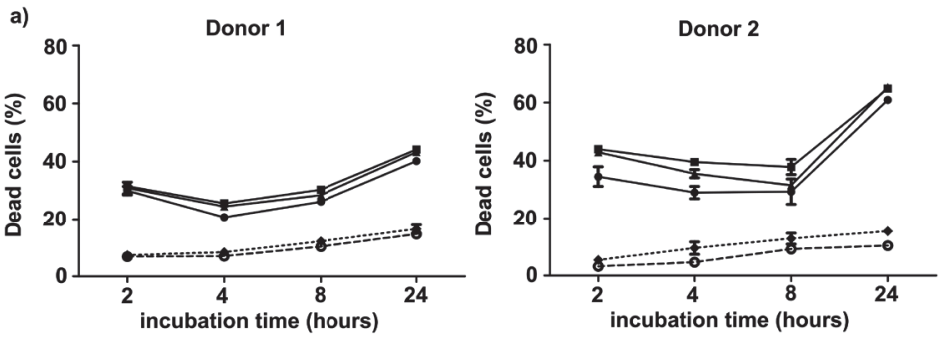

b)

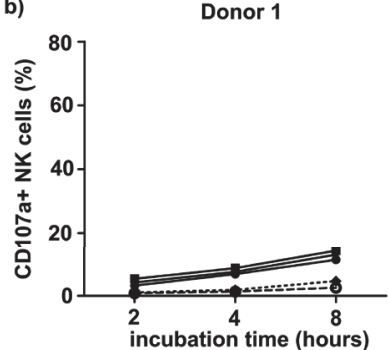

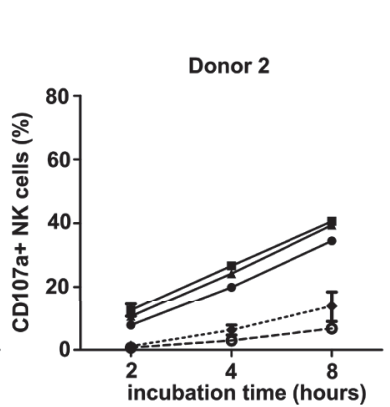

$\rightarrow$ No Antibody

$\rightarrow+$ Daratumumab $0.1 \mu \mathrm{g} / \mathrm{mL}$

-+ Daratumumab $1 \mu \mathrm{g} / \mathrm{mL}$

-+ Daratumumab $10 \mu \mathrm{g} / \mathrm{mL}$

+ + Trastuzumab $1 \mu \mathrm{g} / \mathrm{mL}$

Figure 5. The kinetics of NK cell death and activation in the absence or presence of daratumumab. IL-2 activated NK cells were incubated with or without $0.1 \mu \mathrm{g} / \mathrm{mL}, 1 \mu \mathrm{g} / \mathrm{mL}, 10 \mu \mathrm{g} / \mathrm{mL}$ daratumumab or $1 \mu \mathrm{g} / \mathrm{mL}$ trastuzumab for $2,4,8$, or 24 hours in $21 \% \mathrm{O}_{2}$. a The percentage of dead NK cells was calculated by the percentage of NK cells positive for Live/Dead ${ }^{\circledR}$ Marker. b NK cell degranulation was determined as the percentage of CD107a+ NK cells. Shown are dots representing the mean of replicate cultures with standard deviation. 


\section{Selection of KIR-ligand mismatched donors can help to potentiate NK cell anti-MM reactivity in the TME}

After showing that triggering $A D C C$ is a potent way to enhance NK cell anti-MM reactivity in the presence of TMEF, we questioned whether the selection of KIR-ligand mismatched donors could help to further augment the response. We performed a CD107a assay and determined degranulation of NKG2A negative NK cells (KIR-ligand matched vs mismatched subsets) against CD38-high UM9 or RPMI8226/s cells under different TMEFs and in the absence or presence of daratumumab. The addition of daratumumab to the culture enhanced NK cell degranulation of both KIR-ligand matched and mismatched NK cell subsets upon co-culture with UM9 or RPMI8226/s as compared to conditions without daratumumab (Fig. 6 and supplementary Fig. 5). For KIR-ligand matched NK cells, the average increase by daratumumab was from $6.03 \%$ to $45.87 \%$ under hypoxia ( $p=0.0039$ ), $2.34 \%$ to $29.62 \%$ under hypoxia and lactate $(p=0.0039)$, and $4.05 \%$ to $37.38 \%$ under hypoxia and PGE2 ( $p=0.0039)$. For KIR-ligand mismatched NK cells, the average increase was from $14.32 \%$ to $52.96 \%$ under hypoxia ( $p=0.0039), 4.76 \%$ to $35.17 \%$ under hypoxia and lactate $(p=0.0039)$, and $10.76 \%$ to $46.44 \%$ under hypoxia and PGE2 ( $p=0.0039$ ). We did not observe a difference in the percentage of degranulating NK cells for subsets single positive for KIR2DL1, KIR2DL2/3 or KIR3DL1 (supplementary Fig. 6 and supplementary Fig. 7).

In the absence of daratumumab, the average percentage of degranulating NK cells was higher for the KIR-ligand mismatched subset as compared to the matched subset for all donors tested in response to UM9 or RPMI8226/s. This was observed under hypoxia ( $p=0.0039)$; hypoxia and lactate $(p=0.0091)$; and hypoxia and PGE2 $(p=0.0091)$ ( $p=0.0039$ for all three TME conditions) (Fig. 6a). In the presence of daratumumab, there was little difference between degranulation of the KIR-ligand matched and mismatched subsets (Fig. 6b). This suggests that lowering the activation threshold by KIR-ligand mismatching would be most effective under conditions where the NK cell receives limited activating signals. Furthermore, for both the KIR-ligand mismatched as well as the matched subset, the percentage of degranulating NK cells in response to UM9 or RPMI8226/s with daratumumab was not significantly different between the TME conditions. 
Daratumumab augments alloreactive natural killer cell cytotoxicity towards CD38+ multiple myeloma cell lines in a biochemical context mimicking tumour

microenvironment conditions

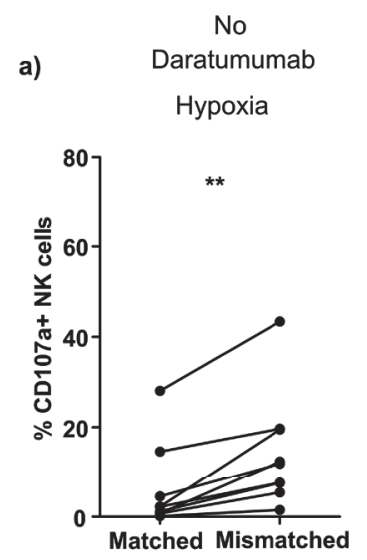

With
Daratumumab
Hypoxia

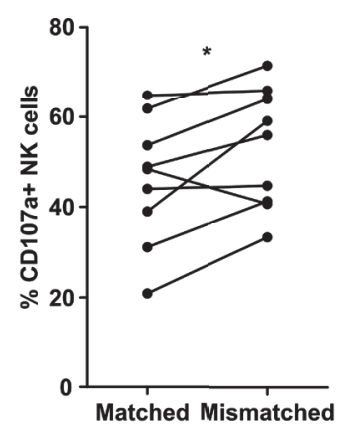

Hypoxia + Lactate

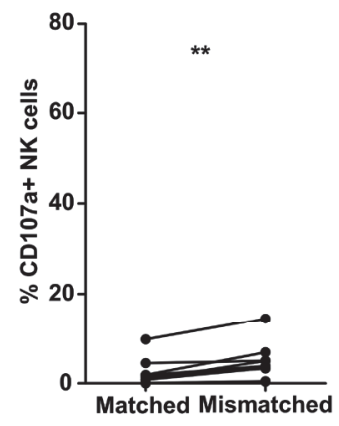

Hypoxia + Lactate

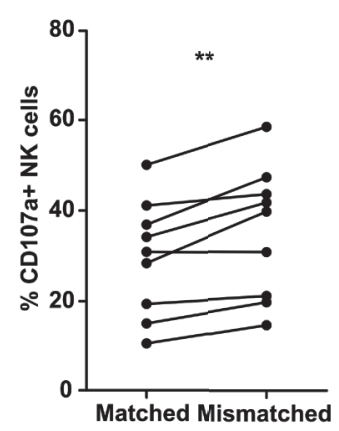

Hypoxia + PGE2
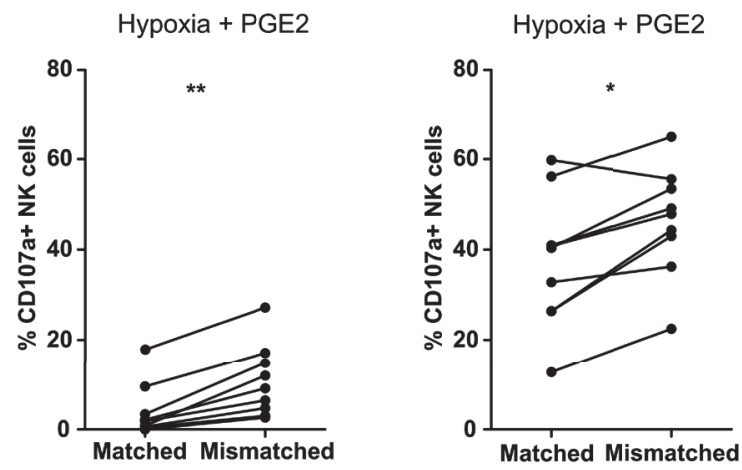

Figure 6. Comparison of degranulation of KIR-ligand mismatched NK cells and KIR-ligand matched NK cells in response to MM cells with or without daratumumab. Following an overnight incubation under hypoxia $\left(0.6 \% \mathrm{O}_{2}\right), \mathrm{UM} 9$ and RPMI8226/s cells were incubated a without or $\mathbf{b}$ with daratumumab for 30 min while IL-2 activated NK cells were incubated for $1 \mathrm{~h}$ with PGE2 or lactate. The percentage of degranulating KIR-ligand matched or KIR-ligand mismatched NK cells was determined as \% CD107a+ cells. Dots represent means of replicate cultures of independent donors. $\mathrm{n}=5$ independent experiments 


\section{DISCUSSION}

In this study, we set out to explore whether the combination of alloreactive NK cells and clinical antibodies targeting tumor-specific/-associated antigens could help to overcome the detrimental effects of the immunosuppressive TME. First, we demonstrated that the NK cell antitumor response can be potentiated by clinicalgrade antibodies and that this was effective even under conditions reflective of an NK cell suppressive TME. Additionally, we provide data demonstrating that selection of KIR-ligand mismatched NK cell donors could help to further amplify the NK cell response.

In our previous study, we showed that hypoxia alone could inhibit NK cell antiMM activity and we demonstrated that IL-2 activation of NK cells could overcome this issue [4]. In this study, we aimed to investigate the influence of a more severe NK-suppressive TME. We observed the inhibitory effect of lactate on NK cell killing already at a low (5mM) concentration (supplementary Fig. 1) which was in line with earlier studies [7]. PGE2 has been shown to negatively impact the NK cell antitumor response [6]. In our hands, NK cell inhibition by PGE2 was less pronounced than with lactate and seemed to be more cell line dependent. In a mouse myeloma model, oxygen levels of $<10 \mathrm{mmHg}(1.3 \%)$ have been shown [24]. In patients with MM, the accumulation of hypoxia-inducible factor-1a (HIF-1a) in bone marrow (BM) biopsies suggested the presence of a hypoxic region in the BM $[25,26]$ and in other tumors, hypoxia frequently coincides with elevated lactate levels. Exact lactate and PGE2 levels in MM BM should, however, be determined to confirm the relevance of these factors for MM. Another important point to consider in more detail in the future is tumor heterogeneity. Although we already performed our assays using multiple cell lines, it is necessary to perform follow up studies with more heterogeneous primary myeloma cells. In vivo, the tumor and the TME will be more complex, and potentially more immunosuppressive which necessitates additional clinical studies in human patients. Nevertheless, our study already illustrated that IL-2 activation alone was not enough to potentiate NK cells which clearly emphasized the need for further activation of NK cells to overcome the inhibitory effect of multiple TMEFs.

In this study, we demonstrated that daratumumab enhanced NK cell-mediated killing of cancer cells under hypoxia, hypoxia/lactate, and hypoxia/PGE2. In line with a previous study [27], we showed that the effect of daratumumab, was specific for MM lines expressing relatively high levels of CD38 (i.e. UM9 and RPMI8226/s). We did not observe $A D C C$ in response to $C D 38$ low or negative cells, suggesting that the level of antigen expression on target cells could affect the outcome. Our observation 
microenvironment conditions

that CD38 expression by MM cells was not reduced by TMEFs, and that binding of daratumumab was not affected by any of the TME conditions, was critical since CD38 expression was required for daratumumab to be effective. Previously, we and others showed that hypoxia alone could slightly reduce CD16 expression [4, 5]. Here, we did not observe a decrease in ADCC capacity under hypoxia alone (Fig. 3), so apparently, this lower CD16 expression did not influence the capacity of NK cells to mediate ADCC. In line with our TME data, daratumumab has previously been shown to potentiate PBMC mediated anti-MM reactivity in the presence of immunosuppressive BM stromal cells and in a mouse model of MM [28]. Together with our current data, this emphasizes the potency of an approach using an ADCC triggering antibody to potentiate NK cells in the TME.

The current availability of a large number of clinically available antibodies which bind to tumor-specific/associated antigens (e.g. cetuximab, rituximab, trastuzumab) on a variety of tumors provides the great opportunity to combine NK cell and antibody therapies. A major challenge, however, is to find antibodies that exclusively bind to tumor cells. Antibodies, such as daratumumab, also interact with antigens expressed on healthy cells, and could give rise to off-target cytotoxicity. We indeed observed a high number of dead or degranulating NK cells in conditions where daratumumab but no tumor target cells were present. Supporting this, in a previous study in MM patients, administration of daratumumab has been shown to decrease the number of peripheral blood NK cells $[29,30]$. These clinical data and our current data could be explained by the fact that NK cells express CD38 [31]. Binding of daratumumab to CD38+ NK cells could trigger ADCC of NK cells against NK cells with bound daratumumab, a phenomenon called fratricide which would be in line with our degranulation data. Elotuzumab, an antibody against CS1 expressed on NK cells and MM cells, has been shown to directly activate NK cells [29]. Presumably, this is not the mechanism for daratumumab as a $\mathrm{CD} 38-\mathrm{F}\left(\mathrm{ab}^{\prime}\right)_{2}$ fragment did not trigger direct NK cell activation [32]. A reduction in NK cell numbers, upon administration to patients, could be detrimental on the long term and the treatment can possibly be further optimized by incubating NK cells, before infusion, with a $\operatorname{CD} 38-F\left(a b^{\prime}\right)_{2}$ fragment to reduce binding of the ADCC mediating antibody.

KIR-ligand mismatched NK cells were more effective against MM target cells than matched cells under all TME conditions tested in this study. Degranulation of both subsets was enhanced by daratumumab and although the difference between matched and mismatched NK cells was less distinct upon addition of daratumumab, mismatched NK cells seemed to be slightly better effector cells. The difference between matched and mismatched cells was not caused by intrinsic differences 
between the subsets as, in response to HLA class I negative K562 cells, all subsets degranulated to the same extent (supplementary Fig. 7). KIR/HLA interactions have been shown to reduce ADCC mediated by rituximab [16, 19, 20, 33]. One of these studies showed that inhibitory KIR/HLA interactions could be compensated for by modification of the Fc part of the rituximab antibody by glycoengineering resulting in an antibody called GA101 (obinutuzumab) with an enhanced potential to trigger ADCC [33]. An alternative approach that has been proposed, is to use the KIR blocking antibodies that are currently available in clinical grade format [20]. Unfortunately, a recent study showed that the administration of anti-KIR antibody in patients with smoldering MM had to be terminated as it resulted in NK cell anergy caused by the removal of KIR receptor from NK cell surface by trogocytosis [34]. In the current study, we demonstrate that selection of a donor based on HLA genotype and KIR expression could be a good way to achieve a KIR-ligand mismatched status to minimize the detrimental effects of KIR/HLA. Selection of KIR-ligand mismatched donors would be feasible for allogeneic NK cell treatments where the patient lacks at least one of the HLA epitopes binding to inhibitory KIRs, as is the case in approximately $70 \%$ of the individuals (Mahaweni unpublished data and [35]). Also in the situation where a glycoengineered antibody is used, selection of a KIR-ligand mismatched donor could be beneficial since tumor cells could downregulate the expression of the antigen targeted by the antibody. In that case, the KIR-ligand mismatch will still facilitate the response against antigen negative cells.

In summary, we showed in this study that the combination of an ADCC triggering antibody and selection of KIR-ligand mismatched donors is a potent and realistic platform to potentiate the NK cell antitumor response in the TME. The difference between allogeneic and non-allogeneic NK cells has to be explored in vivo. The antitumor potential of NK cells can be applied by donor NK cell infusion as well as by haploidentical stem cell transplantation (HaploSCT). In HaploSCT, donor-derived NK cells have been identified as the main mediators of antitumor reactivity and due to the improved post-transplant treatment regimen, HaploSCT is now a realistic and feasible treatment option. Therefore, we envision that the combination of ADCC triggering antibodies and KIR-ligand mismatching is a favorable combination to be tested in future clinical studies, both in the context of HaploSCT, potentially in combination with NK cell infusions, as well as single donor NK cell infusion. 
Daratumumab augments alloreactive natural killer cell cytotoxicity towards CD38+ multiple myeloma cell lines in a biochemical context mimicking tumour

microenvironment conditions

\section{ACKNOWLEDGEMENT}

The authors would like to thank Nick van Dijk for initiating the lactate experiment, Benedict Matern for reviewing English grammar, and the Department of Transplantation Immunology, Maastricht University Medical Center+ for performing the genotyping.

\section{CONFLICT OF INTEREST}

G. M. J. Bos is Chief Executive Officer/Chief Medical Officer/Co-founder of CiMaas, BV, Maastricht, the Netherlands. CiMaas is producing an ex vivo-expanded NK cell product that will be used to treat myeloma patients. The other authors declare no conflict of interest.

\section{FUNDING}

This study was funded by a grant from Kankeronderzoeksfonds Limburg (KOFL). L. Wieten was supported by a grant from Dutch Cancer Association (KWF kankerbestrijding; UM2012-5375). 


\section{REFERENCES}

1. Vivier E, Ugolini S, Blaise D, et al (2012) Targeting natural killer cells and natural killer T cells in cancer. NatRevImmunol 12:239-252. doi: 10.1038/nri3174

2. Sun Y (2016) Tumor microenvironment and cancer therapy resistance. Cancer Lett 380:205-215. doi: 10.1016/j.canlet.2015.07.044

3. Junttila MR, de Sauvage FJ (2013) Influence of tumour micro-environment heterogeneity on therapeutic response. Nature 501:346-354. doi: 10.1038/nature12626

4. Sarkar S, Germeraad WT V, Rouschop KMA, et al (2013) Hypoxia induced impairment of NK cell cytotoxicity against multiple myeloma can be overcome by IL-2 activation of the NK cells. PLoS One 8:e64835. doi: 10.1371/journal.pone.0064835

5. Balsamo M, Manzini C, Pietra G, et al (2013) Hypoxia downregulates the expression of activating receptors involved in NK-cell-mediated target cell killing without affecting ADCC. Eur J Immunol 43:2756-2764. doi: 10.1002/eji.201343448

6. Pietra G, Manzini C, Rivara S, et al (2012) Melanoma cells inhibit natural killer cell function by modulating the expression of activating receptors and cytolytic activity. Cancer Res 72:14071415. doi: 10.1158/0008-5472.CAN-11-2544

7. Husain Z, Huang Y, Seth P, Sukhatme VP (2013) Tumor-Derived Lactate Modifies Antitumor Immune Response: Effect on Myeloid-Derived Suppressor Cells and NK Cells. J Immunol 191:1486-1495. doi: 10.4049/jimmunol.1202702

8. Brand A, Singer K, Koehl GE, et al (2016) LDHA-Associated Lactic Acid Production Blunts Tumor Immunosurveillance by T and NK Cells. Cell Metab 24:657-671. doi: 10.1016/j.cmet.2016.08.011

9. Wang W, Guo H, Geng J, et al (2014) Tumor-released galectin-3, a soluble inhibitory ligand of human NKp30, plays an important role in tumor escape from NK cell attack. J Biol Chem 289:33311-33319. doi: 10.1074/jbc.M114.603464

10. Kopp H-G, Placke T, Salih HR (2009) Platelet-Derived Transforming Growth Factor- DownRegulates NKG2D Thereby Inhibiting Natural Killer Cell Antitumor Reactivity. Cancer Res 69:7775-7783. doi: 10.1158/0008-5472.CAN-09-2123

11. Viel S, Marcais A, Guimaraes FS-F, et al (2016) TGF- inhibits the activation and functions of NK cells by repressing the mTOR pathway. Sci Signal 9:ra19-ra19. doi: 10.1126/scisignal.aad1884

12. Hoechst B, Voigtlaender T, Ormandy L, et al (2009) Myeloid derived suppressor cells inhibit natural killer cells in patients with hepatocellular carcinoma via the NKp30 receptor. Hepatology 50:799-807. doi: 10.1002/hep.23054

13. Mao Y, Sarhan D, Steven A, et al (2014) Inhibition of tumor-derived prostaglandin-E2 blocks the induction of myeloid-derived suppressor cells and recovers natural killer cell activity. Clin Cancer Res 20:4096-4106. doi: 10.1158/1078-0432.CCR-14-0635

14. Bryceson YT, March ME, Ljunggren H-G, Long EO (2006) Synergy among receptors on resting NK cells for the activation of natural cytotoxicity and cytokine secretion. Blood 107:159-166. doi: 10.1182/blood-2005-04-1351

15. Seidel UJE, Schlegel P, Lang P (2013) Natural Killer Cell Mediated Antibody-Dependent Cellular Cytotoxicity in Tumor Immunotherapy with Therapeutic Antibodies. Front Immunol 4:76. doi: 10.3389/fimmu.2013.00076

16. Wang W, Erbe AK, Hank JA, et al (2015) NK Cell-Mediated Antibody-Dependent Cellular Cytotoxicity in Cancer Immunotherapy. Front Immunol 6:368. doi: 10.3389/fimmu.2015.00368

17. Nijhof IS, Groen RWJ, Noort WA, et al (2015) Preclinical evidence for the therapeutic potential of CD38-Targeted Immuno-chemotherapy in multiple Myeloma patients refractory to Lenalidomide and Bortezomib. Clin Cancer Res 21:2802-2810. doi: 10.1158/1078-0432.CCR-141813 


\section{microenvironment conditions}

18. Sarkar S, van Gelder M, Noort W, et al (2015) Optimal selection of natural killer cells to kill myeloma: the role of HLA-E and NKG2A. Cancer Immunol Immunother 64:951-963. doi: 10.1007/s00262-015-1694-4

19. Kohrt HE, Thielens A, Marabelle A, et al (2014) Anti-KIR antibody enhancement of anti-lymphoma activity of natural killer cells as monotherapy and in combination with anti-CD20 antibodies. Blood 123:678-686. doi: 10.1182/Blood-2013-08-519199

20. Binyamin L, Alpaugh RK, Hughes TL, et al (2008) Blocking NK cell inhibitory self-recognition promotes antibody-dependent cellular cytotoxicity in a model of anti-lymphoma therapy. J Immunol 180:6392-6401.

21. McMillin DW, Delmore J, Weisberg E, et al (2010) Tumor cell-specific bioluminescence platform to identify stroma-induced changes to anticancer drug activity. Nat Med 16:483-489. doi: $10.1038 / \mathrm{nm} .2112$

22. Walenta S, Wetterling M, Lehrke M, et al (2000) High lactate levels predict likelihood of metastases, tumor recurrence, and restricted patient survival in human cervical cancers. Cancer Res 60:916-921.

23. Hidalgo GE, Zhong L, Doherty DE, Hirschowitz E a (2002) Plasma PGE-2 levels and altered cytokine profiles in adherent peripheral blood mononuclear cells in non-small cell lung cancer (NSCLC). Mol Cancer 1:5. doi: 10.1186/1476-4598-1-5

24. Hu J, Van Valckenborgh E, Menu E, et al (2012) Understanding the hypoxic niche of multiple myeloma: therapeutic implications and contributions of mouse models. Dis Model Mech 5:763771. doi: 10.1242/dmm.008961

25. Martin SK, Diamond P, Williams SA, et al (2010) Hypoxia-inducible factor-2 is a novel regulator of aberrant CXCL12 expression in multiple myeloma plasma cells. Haematologica 95:776-784. doi: 10.3324/haematol.2009.015628

26. Giatromanolaki A, Bai M, Margaritis D, et al (2010) Hypoxia and activated VEGF/receptor pathway in multiple myeloma. Anticancer Res 30:2831-6.

27. Sanchez L, Wang Y, Siegel DS, Wang ML (2016) Daratumumab: a first-in-class CD38 monoclonal antibody for the treatment of multiple myeloma. J Hematol Oncol 9:51. doi: 10.1186/s13045016-0283-0

28. Weers M de, Tai Y-T, Veer MS van der, et al (2011) Daratumumab, a Novel Therapeutic Human CD38 Monoclonal Antibody, Induces Killing of Multiple Myeloma and Other Hematological Tumors. J Immunol 186:1840-1848. doi: 10.4049/jimmunol.1003032

29. Phipps C, Chen Y, Gopalakrishnan S, Tan D (2015) Daratumumab and its potential in the treatment of multiple myeloma: overview of the preclinical and clinical development. Ther Adv Hematol 6:120-7. doi: 10.1177/2040620715572295

30. McEllistrim C, Krawczyk J, O'Dwyer ME (2017) New developments in the treatment of multiple myeloma - clinical utility of daratumumab. Biologics 11:31-43. doi: 10.2147/BTT.S97633

31. Krejcik J, Casneuf T, Nijhof IS, et al (2016) Daratumumab depletes CD38+ immune regulatory cells, promotes T-cell expansion, and skews T-cell repertoire in multiple myeloma. Blood 128:384-394. doi: 10.1182/blood-2015-12-687749

32. Elena Cherkasova, Luis Espinoza, Ritesh Kotecha, Robert N. Reger, Maria Berg, Georg Aue, Ricardo M. Attar, A Kate Sasser, Mattias Carlsten RWC (2015) Treatment of Ex Vivo-Expanded NK Cells with Daratumumab F(ab')2 Fragments Protects Adoptively Transferred NK Cells from Daratumumab-Mediated Killing and Augments Daratumumab-Induced Antibody Dependent Cellular Toxicity (ADCC) of Myeloma. Blood 126:4244.

33. Terszowski G, Klein C, Stern M (2014) KIR/HLA Interactions Negatively Affect Rituximab- but Not GA101 (Obinutuzumab)-Induced Antibody-Dependent Cellular Cytotoxicity. J Immunol 192:5618-24. doi: 10.4049/jimmunol.1400288 
34. Carlsten M, Korde N, Kotecha R, et al (2016) Checkpoint inhibition of KIR2D with the monoclonal antibody IPH2101 induces contraction and hyporesponsiveness of NK cells in patients with myeloma. Clin Cancer Res 22:5211-5222. doi: 10.1158/1078-0432.CCR-16-1108

35. Omar SY Al, Alkuriji A, Alwase S, et al (2016) Genotypic diversity of the killer cell immunoglobulinlike receptors (KIR) and their HLA class i ligands in a saudi population. Genet Mol Biol 39:14-23. doi: 10.1590/1678-4685-GMB-2015-0055 


\section{SUPPLEMENTARY FIGURES}

a)
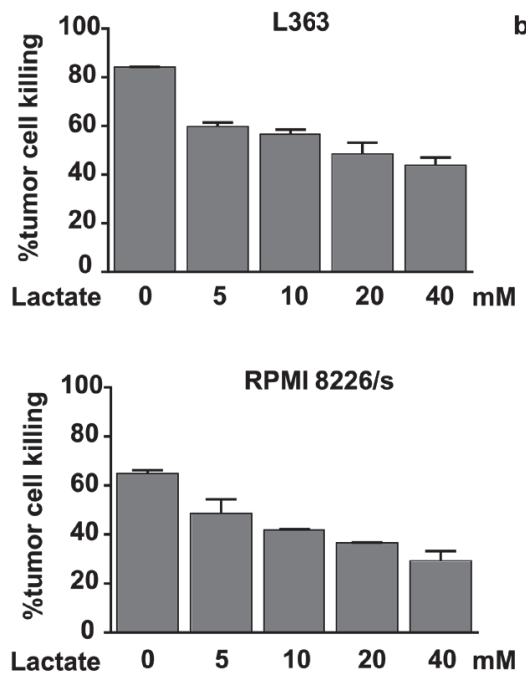

b)
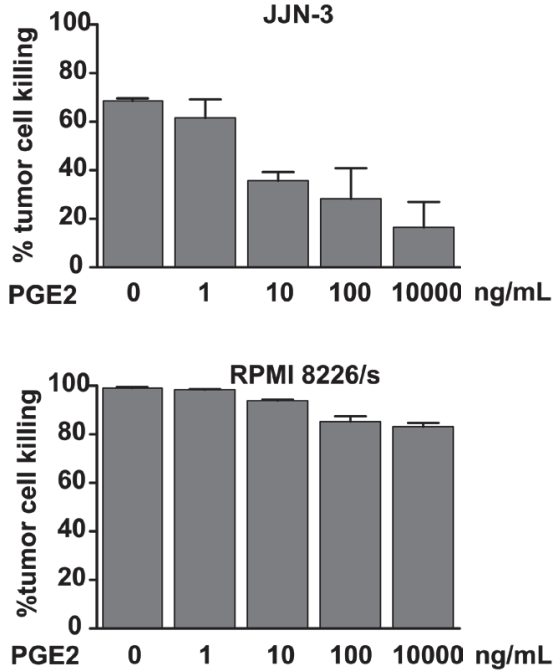

Supplementary Figure 1. Effect of lactate and PGE2 on the killing capacity of NK cells. In a compartment specific bioluminescence imaging (CS-BLI) based assay, luciferase-expressing L363, JJN-3 or RPMI8226/s cells were incubated overnight with KHYG-1 NK cell line, in the absence or presence of indicated concentrations of a lactate or b PGE2. Shown are the mean and standard deviation (SD) of $n=4$ technical replicates from one experiment.

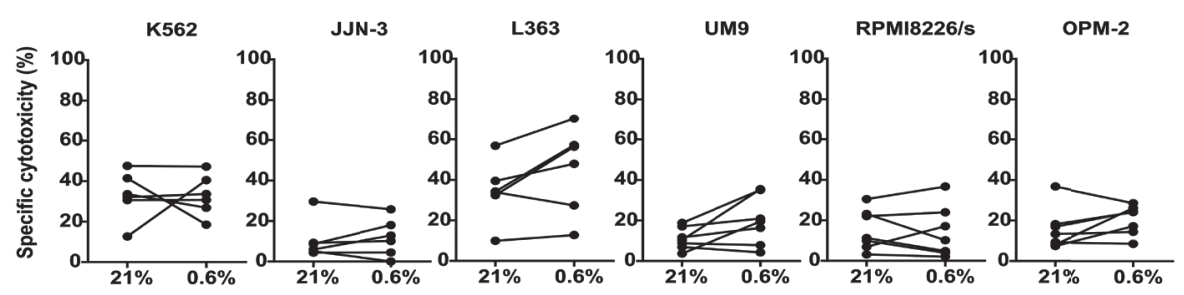

Supplementary Figure 2. Killing of tumor cells by IL-2-activated NK cells under normoxia and hypoxia. IL-2 activated NK cells were co-cultured with Dil-labeled target cells in 1:1 E:T ratio in a 4 hour flow cytometry-based cytotoxicity assay, under $21 \% \mathrm{O}_{2}$ (ambient air) or $0.6 \% \mathrm{O}_{2}$ (hypoxia). Each dot represents the mean of replicate culture of one donor ( $n=5$ donors in 5 independent experiments). 


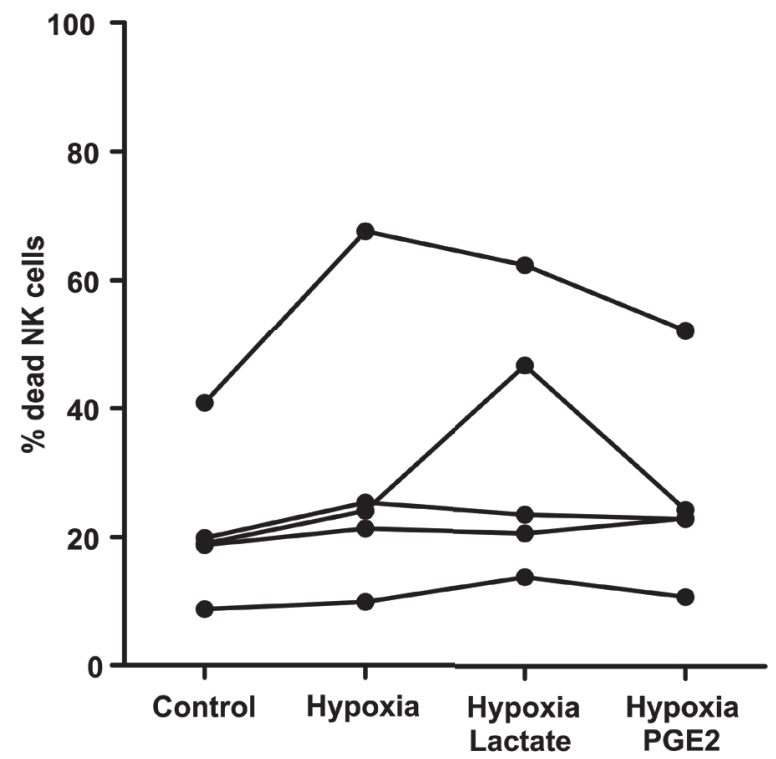

Supplementary Figure 3. Percentage of dead NK cells exposed to TME factors. NK cells were cultured under $21 \% \mathrm{O}_{2}$ only (control), or under hypoxia $\left(0.6 \% \mathrm{O}_{2}\right)$ or in combination of hypoxia with lactate or PGE2 for 5 hours. Dead cells were evaluated by calculating the percentage of NK cells positive for Live/Dead Marker. Per condition, each dot represents the mean of replicate culture of one donor ( $\mathrm{n}=5$ donors in 5 independent experiments).

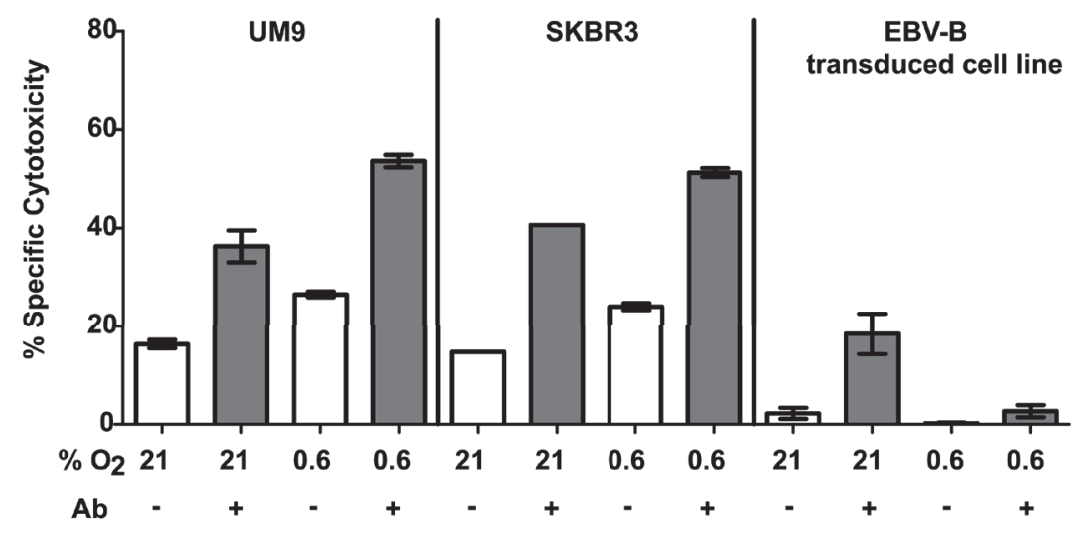

Supplementary Figure 4. Effect of clinical-grade antibodies on NK cell mediated ADCC under hypoxia. Dil-labeled UM9 (CD38+), SKBR3 (HER2+), or EBV-B transduced cell line (CD20+) were incubated overnight at $0.6 \%$ or $21 \% \mathrm{O}_{2}$. The next day, 30 minutes prior to 4 hours flow cytometrybased cytotoxicity assay, tumor cells were incubated with either $1 \mu \mathrm{g} / \mathrm{mL}$ daratumumab (UM9), 1 $\mu \mathrm{g} / \mathrm{mL}$ trastuzumab (SKBR3), or $10 \mu \mathrm{g} / \mathrm{mL}$ rituximab (EBV-B transduced cell line) while IL-2 activated NK cells were incubated for 1 hour with $100 \mathrm{ng} / \mathrm{mL}$ PGE2 or $50 \mathrm{mM}$ lactate. Shown are the mean of replicate culture with SD. $\mathrm{n}=2$ experiments for UM9 and SKBR3, 1 experiment for EBV-B. 
Daratumumab augments alloreactive natural killer cell cytotoxicity towards CD38+ multiple myeloma cell lines in a biochemical context mimicking tumour

a)
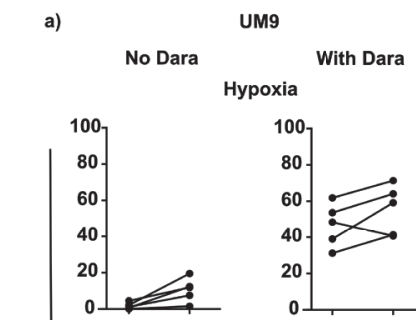

\begin{tabular}{l}
0 \\
$\vdots$ \\
0 \\
\hline 0 \\
0 \\
$\frac{1}{2}$ \\
+ \\
$\vdots$ \\
$\frac{1}{0}$ \\
0 \\
0
\end{tabular}

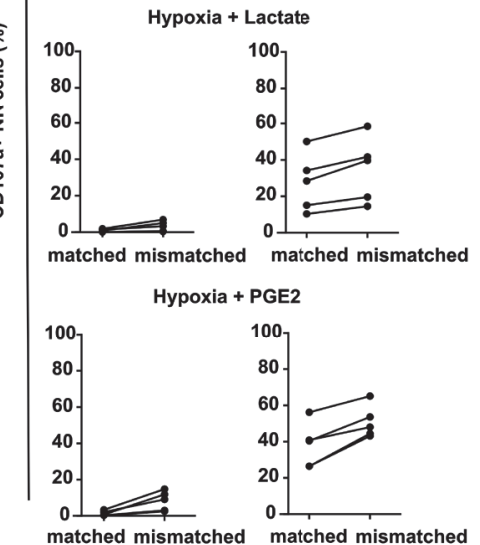

microenvironment conditions

b)

RPMI8226/s

No Dara With Dara

Hypoxia

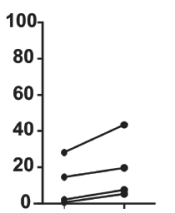

-

matched mismatched matched mismatched

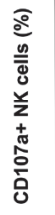

Hypoxia + Lactate

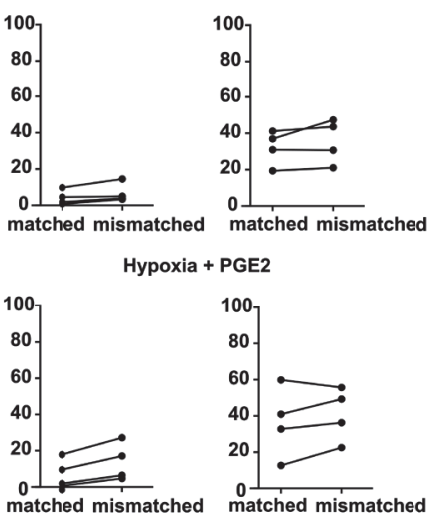

Supplementary Figure 5. Comparison of degranulation of KIR-ligand mismatched NK cells and KIR-ligand matched NK cells in response to MM cells with or without daratumumab. Following an overnight incubation in the presence of hypoxia $\left(0.6 \% \mathrm{O}_{2}\right)$, a UM9 and b RPMI8226/s cells were incubated with daratumumab for 30 minutes while IL-2 activated NK cells were incubated for 1 hour with $100 \mathrm{ng} / \mathrm{mL}$ PGE2 or $50 \mathrm{mM}$ lactate.

Dots represent means of replicate cultures. $n=5$ independent experiments 
Without Daratumumab

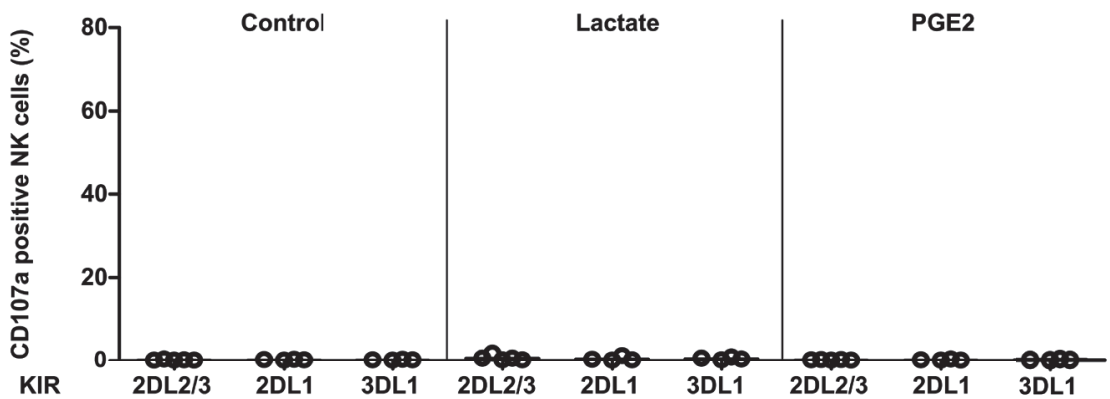

With Daratumumab

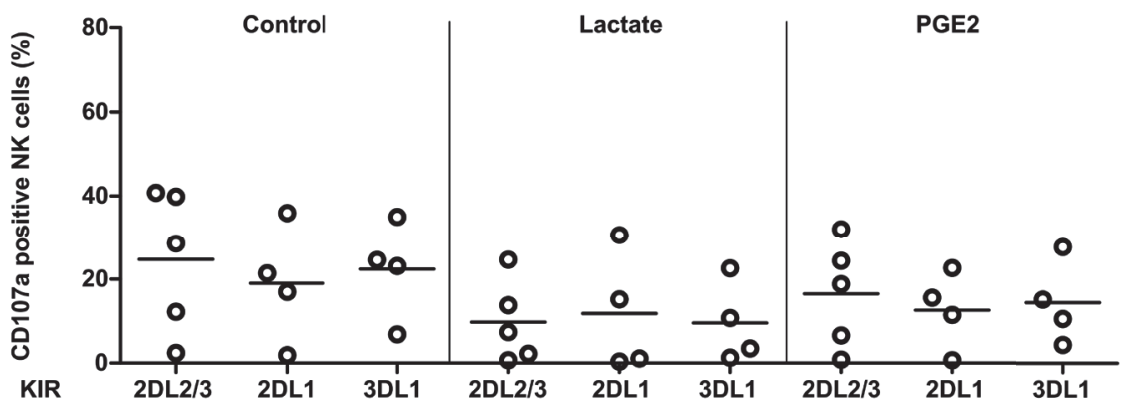

Supplementary Figure 6. Spontaneous degranulation of NK cell subsets. NK cell spontaneous degranulation was measured in a flow cytometry-based degranulation (CD107a) assay after 5 hours incubation of NK cells under hypoxia $\left(0.6 \% \mathrm{O}_{2}\right)$ alone (control) or the combination of hypoxia and lactate or hypoxia and PGE2 in the presence or absence of daratumumab. Each dot represents the mean of replicate culture of one donor ( $n=5$ donors in 5 independent experiments). 
microenvironment conditions

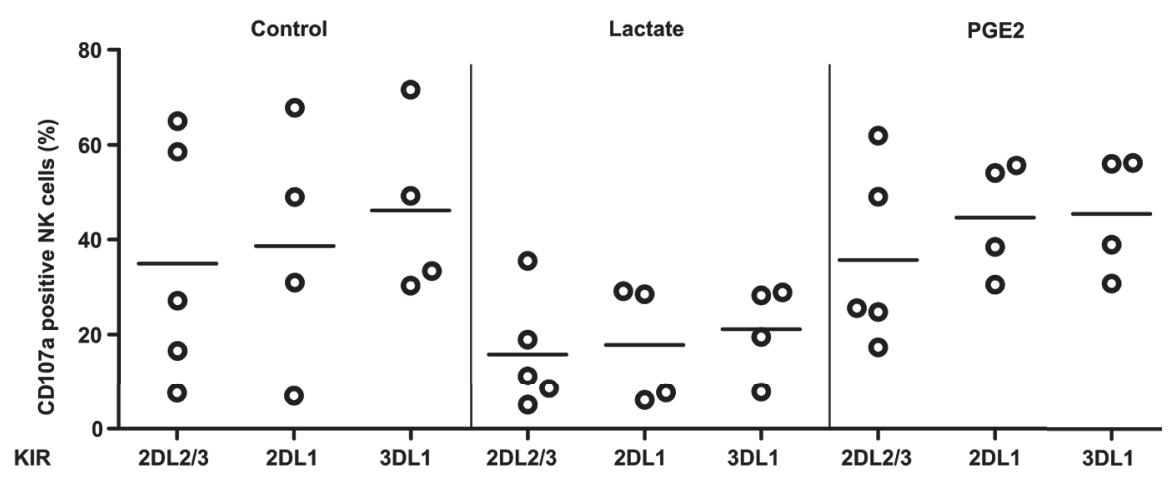

Supplementary figure 7. Degranulating capacity of KIR2DL2/3, KIR2DL1, and KIR3DL1 subsets in the absence of inhibitory signals from HLA class I. NK cells were co-cultured with $\mathrm{K} 562$ cells, in a flow cytometry-based degranulation (CD107a) assay in the presence of hypoxia $\left(0.6 \% \mathrm{O}_{2}\right)$ alone (control) or the combination of hypoxia and lactate or hypoxia and PGE2. Each dot represents the mean of replicate culture of one donor ( $n=5$ donors in 5 independent experiments). 
$62 \mid$ Chapter 3 


\section{A comprehensive overview of FCGR3A gene variability by full- length gene sequencing including the identification of $\mathrm{V} 158 \mathrm{~F}$ polymorphism}

Niken M. Mahaweni1,2, Timo I. Olieslagers1, Ivan Olivares Rivas1, Stefan J. J. Molenbroeck1, Mathijs Groeneweg1, Gerard M. J. Bos2, Marcel G. J. Tilanus1, Christina E. M. Voorter1, Lotte Wieten ${ }^{*}$ *

1 Department of Transplantation Immunology, Tissue Typing Laboratory, GROW School for Oncology and Developmental Biology, Maastricht University Medical Centert, the Netherlands

2 Department of Internal Medicine, division of Hematology, GROW School for Oncology and Developmental Biology, Maastricht University Medical Center+, the Netherlands 


\section{ABSTRACT}

The FCGR3A gene encodes for the receptor important for antibody-dependent natural killer cell-mediated cytotoxicity. FCGR3A gene polymorphisms could affect the success of monoclonal antibody therapy. Although polymorphisms, such as the FcyRIIIA-V158F and $-48 \mathrm{~L} / \mathrm{R} / \mathrm{H}$, have been studied extensively, an overview of other polymorphisms within this gene is lacking. To provide an overview of FCGR3A polymorphisms, we analysed the 1000 Genomes project database and found a total of 234 polymorphisms within the FCGR3A gene, of which $69 \%, 16 \%$, and $15 \%$ occur in the intron, UTR, and exon regions respectively. Additionally, only $16 \%$ of all polymorphisms had a minor allele frequency (MAF) $>0.01$. To facilitate (fulllength) analysis of FCGR3A gene polymorphism, we developed a FCGR3A genespecific amplification and sequencing protocol for Sanger sequencing and MinION (Nanopore Technologies). First, we used the Sanger sequencing protocol to study the presence of the $\mathrm{V} 158 \mathrm{~F}$ polymorphism in 76 individuals resulting in frequencies of $38 \%$ homozygous $\mathrm{T} / \mathrm{T}, 7 \%$ homozygous $\mathrm{G} / \mathrm{G}$ and $55 \%$ heterozygous. Next, we performed a pilot with both Sanger sequencing and MinION-based sequencing of 14 DNA samples which showed a good concordance between Sanger- and MinION sequencing. Additionally, we detected 13 SNPs listed in the 1000 Genome Project, from which 11 had MAF >0.01, and 10 SNPs were not listed in 1000 Genome Project. In summary, we demonstrated that FCGR3A gene is more polymorphic than previously described. As most novel polymorphisms are located in non-coding regions, their functional relevance needs to be studied in future functional studies. 


\section{INTRODUCTION}

Natural killer (NK) cells are innate lymphocytes and pivotal players in the defense against malignant- or virally-infected cells [1]. NK cells can produce cytokines and kill target cells [2]. Moreover, NK cells mediate antibody-dependent cell-mediated cytotoxicity $(A D C C)$ via the ligation of their low affinity Fc receptor, FcyRIIla, also known as CD16, with an antibody bound to a potential target cell [1, 3].

As reviewed recently, the strength of the ADCC response could be determined by several factors, amongst them the isotype-, fucosylation- and glycosylationcharacteristics of the antibody as well as genotypic variation of the FcyRIIla receptor itself [4]. A clear example of the latter is the single nucleotide substitution (SNP) from $\mathrm{G}$ to $\mathrm{T}$ at CDNA nucleotide position 559 of the FCGR3A gene generating two different FcyRIIla allotypes: one with a valine (V) and one with a phenylalanine (F) at amino acid position 158, known as FcyRIIIA-V158F polymorphism (rs396991) [5-7]. The presence of a valine (V/V or V/F) has been shown to enhance the NK cell's binding affinity to an $\lg G 1$ or $\lg G 3$ antibody as compared to the presence of a homozygous phenylalanine genotype (F/F), resulting in a higher level of NK cell-mediated ADCC [6-8].

In antibody-based immunotherapy, NK cell-mediated ADCC is one of the mechanisms underlying the anti-cancer effects of frequently used antibodies like rituximab, trastuzumab, and cetuximab. Several clinical studies provided evidence for the functional relevance of the V158Fpolymorphism in this setting: In non-Hodgkin lymphoma, HER-2/neu-positive metastatic breast cancer, metastatic colorectal cancer or head and neck cancer, patients with V/V polymorphism appeared to have an improved progression-free survival as compared to patients with F/F phenotype [9-13]. Moreover, a study examining rituximab and ADCC in healthy donors suggested that the expression of at least one valine at FcyRIIla-158 could explain the improved clinical outcome [14]. Nonetheless, two other studies [15, 16] did not find any correlation between the V158F polymorphism and the clinical outcome possibly due to sample size limitation.

The characterization of the FCGR3A gene polymorphism may also be relevant in the solid organ transplantation setting where, in the presence of antibodies against a renal graft, NK cells have been shown to mediate ADCC contributing to graft rejection $[17,18]$. A recent study on cardiac allograft showed that patients with V/V genotype had an enhanced CD16 expression and were associated with a higher risk of developing vasculopathy and eventually allograft rejection [19]. 
Interestingly, a study on bone marrow transplantation for myeloid malignancies suggested that the $\mathrm{V} 158 \mathrm{~F}$ polymorphism in recipients could predict transplant outcomes and the presence of V/V genotype in recipients was associated with a significantly reduced risk of acute and chronic graft-versus-host disease as well as better overall survival [20]. Furthermore, patients with F/F or V/F genotype have been shown to have a higher predisposition to an increased incidence of infection after liver transplantation [21].

In addition to the V158F polymorphism, several additional polymorphisms in the FCGR3A gene have been identified: 1) the FcyRIIIA-48L/R/H polymorphism (rs10127939), where a single nucleotide substitution from $\mathrm{T}$ to $\mathrm{G}$ is responsible for a leucine $(L)$ to an arginine $(R)$ substitution and $T$ to $A$ is responsible for a leucine $(L)$ to a histidine $(\mathrm{H})$ at amino acid position 48 . Both these substitutions have been reported to have an enhanced binding to the $\operatorname{lgG}_{1}, \operatorname{lgG}_{3^{\prime}}$ and $\operatorname{lgG}_{4}$ [22]. This polymorphism has also been demonstrated to be linked to the FcyRIIIA-V158F polymorphism [6] where the FcyRIIIA-48L/R/H polymorphism influenced ligand binding capacity in the presence of the FcyRIIIA-V158F polymorphism [23]. The presence of $\mathrm{R}$ or $\mathrm{H}$ allele and at least one copy of $V$ allele provided a higher binding capacity. 2) A homozygous missense mutation in the FCGR3A gene encoding an $\mathrm{L} 48 \mathrm{H}$ substitution causing a defect in NK cell cytotoxicity due to a reduced surface expression of CD2, a coactivation receptor, while preserving an intact ADCC [24]. 3) Two SNPs (rs4656317 and rs12071048) located within the enhancer region of the FCGR3A gene that are in strong linkage disequilibrium with the FcyRIIIA-V158F polymorphism and strongly affected NK cell ADCC activity where the major alleles had a higher ADCC activity than those with minor alleles [25], 4) a 3-SNP/1-indel FCGR3A intragenic haplotype which was associated with increased FcyRIlla expression [26]. 5) Several other polymorphisms in the FCGR3A gene, i.e. rs2099684 [27]; rs10919543 [28]; and rs445509 [29], that have been found to be associated with arteritis [27, 28] and chronic periodontitis [29].

The above mentioned studies highlighted the potential relevance of FCGR3A polymorphisms for NK cell effector function and their potential clinical relevance. However, the analysis is frequently complicated by the presence of FCGR3B gene, encoding the inhibitory FcyRIIlb receptor, as the FCGR3B gene is highly homologous to the FCGR3A gene except that it has a T at nucleotide 531 of the cDNA instead of a $C[7,30]$. Another issue is that previous methods were focused on sequencing particular exons of the gene $[7,31]$ while extended polymorphism in for example $5^{\prime}$ or $3^{\prime}$ UTR or in introns could also influence CD16 expression e.g. by influencing micro-RNA binding or alternative splicing [32]. To facilitate future studies to unravel the functional consequences of full length CD16 polymorphism, we established a 
standardized way to determine V158F gene polymorphism using Sanger sequencing and we tested a new full-length gene single molecule sequencing method for the identifcation of polymorphism in the FCGR3A gene using MinION (a Nanopore technology). We subsequently used these methods, combined with the data present for the FCGR3A gene in the database of phase 3 of the 1000 Genomes project (1KGP), to generate a more comprehensive overview of full-length CD16a polymorphism. 


\section{RESULTS}

\section{FCGR3A gene variability beyond the V158F polymorphism}

To study the magnitude of FCGR3A gene polymorphism, we analysed the nucleotide variability data available in the $1 \mathrm{KGP}$ for this gene and mapped all the polymorphisms identified in $1 \mathrm{KGP}$ based on the location and the minor allele frequency (MAF) (Fig .1). The polymorphic index (PI), the number of polymorphic positions divided by the length of the region, of the whole gene and of the individual introns/exons/UTR were calculated (Table 1). This illustrated that exon 3 is the most polymorphic region in the gene, with a PI of 0.066 , while exon 2 has the lowest PI. The gene sequence of exon 2-5 encodes for the FcyRIIla receptor, which consists of an extracellular domain with two Ig-like domains (exon 3 and 4) and five potential N-glycosylation sites (three in exon 3 and two in exon 4), a transmembrane domain (exon 5) and a cytoplasmic domain (exon 5).

A total of 234 polymorphisms (3\% of the entire gene, SNP density: 2.83 SNP/100bp) were identified, of which $34(15 \%)$ are present in the exons, $162(69 \%)$ in the introns, and $38(16 \%)$ in the untranslated regions (UTRs) (Fig. 2). Of note, only 36 of these 234 polymorphisms have a MAF higher than $1 \%$ (Table 2). A relatively high number of the polymorphisms are located in intron 3 as compared to the other regions (16 out of 36).

Of the 34 polymorphisms identified in the exons, 22 (65\%) are non-synonymous and 12 (35\%) are synonymous. Only one non-synonymous (rs10127939 C/T) and two synonymous polymorphisms (rs114535887 and rs150808747) have a MAF greater than $1 \%$. The non-synonymous polymorphism is located at nucleotide position 1302 in exon 3 with three different nucleotides possible ( $T, G$, and $A)$, resulting in three different amino acids: leucine ( $L, M A F 0.09)$, arginine (, , MAF 0.039), and histidine ( $H$, MAF 0.027 ) and three different alleles. The synonymous polymorphism are located at nucleotide position 1321 and 1336 and have a MAF of 0.019 , and 0.012 respectively. The V158F polymorphism at nucleotide position 5093 ( $r$ 396991) is not documented in the $1 \mathrm{KG}$ because it did not reach the quality control threshold, most probably because of its location in a homopolymer-rich region, and thus no frequency information of this polymorphism was attainable. For this reason, Figure 1 shows an arrow demonstrating the location of the V/F polymorphism, but provides no further information. 


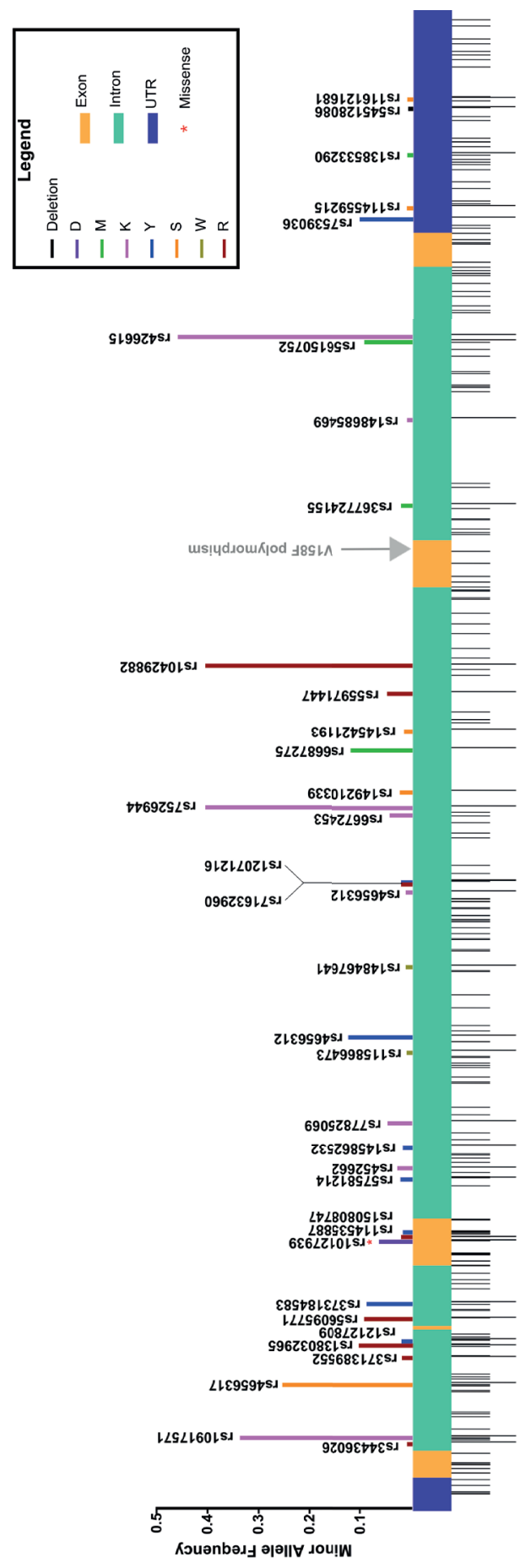

Figure 1. Schematic illustration of FCGR3A gene and its 234 polymorphisms according to the 1000 Genome database. The stripes present underneath represent different polymorphisms. All polymorphisms with MAF $>0.01$ are shown on the upper part of the scheme and the rs number is shown. Different colors denote the amino acid changes as shown in the legend. The grey arrow points at the location of the V/F polymorphism. 
Table 1. Number of polymorphisms present in the FCGR3A gene described in the $1 \mathrm{KG}$ database.

\begin{tabular}{cccc} 
Location & Bases & Polymorphisms & PI \\
\hline 5'UTR & 183 & 5 & 0.027 \\
Exon 1 & 147 & 8 & 0.054 \\
Intron 1 & 664 & 30 & 0.045 \\
Exon 2 & 20 & 0 & 0.000 \\
Intron 2 & 331 & 13 & 0.039 \\
Exon 3 & 257 & 17 & 0.066 \\
Intron 3 & 3461 & 87 & 0.025 \\
Exon 4 & 257 & 4 & 0.016 \\
Intron 4 & 1501 & 32 & 0.021 \\
Exon 5 & 186 & 5 & 0.027 \\
3'UTR & 1252 & 33 & 0.026 \\
\hline Coding region & 867 & 34 & 0.039 \\
Noncoding region & 7392 & 200 & 0.027 \\
Whole gene & 8259 & 234 & 0.028
\end{tabular}

The table reports the number of polymorphisms per location and the polymorphic index. $\mathrm{PI}=$ Polymorphic Index.

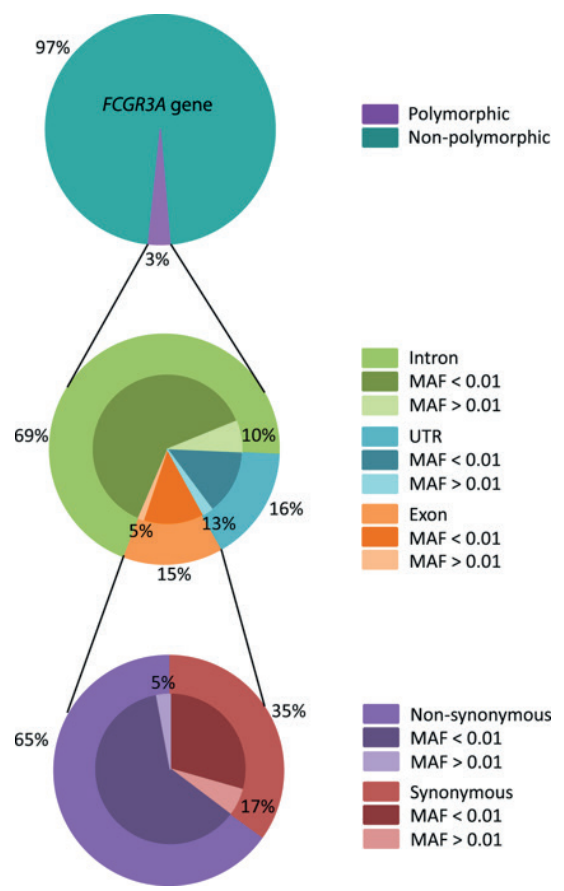

Figure 2. Schematic overview of overall polymorphisms in the FCGR3A gene in the 1 KG database. The bottom circle depicts the whole FCGR3A gene. The light grey slice shows the percentage of the gene that is polymorphic. 
Table 2. Polymorphisms with a minor allele frequency (MAF) higher than $1 \%$ in the $1 \mathrm{KG}$ project.

\begin{tabular}{|c|c|c|c|c|c|}
\hline SNP & Position in gene & Location & Polymorphism & MAF & Amino Acid Change \\
\hline rs34436026 & 195 & Intron 1 & $\mathrm{R}$ & A: 0.011 & \\
\hline rs10917571 & 224 & Intron 1 & K & $\mathrm{T}: 0.340$ & \\
\hline rs4656317 & 516 & Intron 1 & $S$ & $\mathrm{G}: 0.257$ & \\
\hline rs371389552 & 664 & Intron 1 & $\mathrm{R}$ & $\mathrm{A}: 0.021$ & \\
\hline rs138032965 & 727 & Intron 1 & $\mathrm{R}$ & A: 0.106 & \\
\hline rs12127809 & 756 & Intron 1 & Y & $C: 0.022$ & \\
\hline rs56095771 & 878 & Intron 2 & $\mathrm{R}$ & G: 0.095 & \\
\hline \multirow[t]{2}{*}{ rs373184583 } & 959 & Intron 2 & Y & $\mathrm{T}: 0.090$ & \\
\hline & & & & G: 0.039 & $\mathrm{~L} / \mathrm{R} / \mathrm{H}$ \\
\hline rs10127939 & 1302 & Exon 3 & $D$ & A: 0.027 & \\
\hline rs114535887 & 1321 & Exon 3 & $\mathrm{R}$ & A: 0.019 & \\
\hline rs150808747 & 1336 & Exon 3 & Y & $\mathrm{T}: 0.012$ & \\
\hline rs57581214 & 1641 & Intron 3 & Y & $\mathrm{T}: 0.024$ & \\
\hline rs452662 & 1696 & Intron 3 & K & $\mathrm{T}: 0.030$ & \\
\hline rs 145862532 & 1816 & Intron 3 & Y & $\mathrm{T}: 0.019$ & \\
\hline rs 77825069 & 1950 & Intron 3 & K & $\mathrm{T}: 0.048$ & \\
\hline rs115866473 & 2336 & Intron 3 & W & $\mathrm{T}: 0.012$ & \\
\hline rs4656312 & 2418 & Intron 3 & Y & $\mathrm{T}: 0.126$ & \\
\hline rs 148467641 & 2802 & Intron 3 & W & $\mathrm{T}: 0.014$ & \\
\hline rs 145392761 & 3213 & Intron 3 & K & $\mathrm{T}: 0.014$ & \\
\hline rs71632960 & 3275 & Intron 3 & Y & $\mathrm{T}: 0.023$ & \\
\hline rs12071216 & 3278 & Intron 3 & $\mathrm{R}$ & $\mathrm{G}: 0.018$ & \\
\hline rs7526944 & 3678 & Intron 3 & K & $\mathrm{T}: 0.408$ & \\
\hline rs149210339 & 3763 & Intron 3 & $S$ & $C: 0.025$ & \\
\hline rs 6687275 & 3997 & Intron 3 & M & $C: 0.121$ & \\
\hline rs 145421193 & 4098 & Intron 3 & $S$ & $\mathrm{G}: 0.017$ & \\
\hline rs55971447 & 4308 & Intron 3 & $\mathrm{R}$ & $A: 0.050$ & \\
\hline rs10429882 & 4459 & Intron 3 & $\mathrm{R}$ & $\mathrm{G}: 0.408$ & \\
\hline rs367724155 & 5333 & Intron 4 & $M$ & $C: 0.023$ & \\
\hline rs 148685469 & 5803 & Intron 4 & K & $\mathrm{G}: 0.011$ & \\
\hline rs56150752 & 6229 & Intron 4 & M & C: 0.094 & \\
\hline rs426615 & 6258 & Intron 4 & K & $\mathrm{G}: 0.462$ & \\
\hline rs7539036 & 6904 & 3 'UTR & Y & $\mathrm{T}: 0.107$ & \\
\hline rs114559215 & 6975 & 3 'UTR & $S$ & $C: 0.013$ & \\
\hline rs 138533290 & 7256 & 3 'UTR & K & 0.012 & \\
\hline rs545128086 & 7507 & 3 'UTR & Deletion (A/-) & 0.010 & \\
\hline rs116121681 & 7558 & 3 'UTR & $S$ & 0.012 & \\
\hline
\end{tabular}




\section{Sanger Sequencing for detection of V158F polymorphism in the FCGR3A gene}

To establish a convenient method to analyse FcyRllla-158 polymorphism, while excluding the highly homologous FCGR3B gene, we explored a Sanger sequencing based approach that would also enable analysis of extended polymorphism. The FCGR3A gene sequence of the 1KGP database was used as reference for the sequencing analysis. First, we focused the analysis on the FcyRIIla-158 polymorphism and, in our sequence data, we identified the FCGR3A gene by the presence of a $C$ nucleotide at the position 5065 while a T would be identified in case of an FCGR3B gene (Fig. 3a). The FcyRllla-158 polymorphism variants at nucleotide position 5093 (T/T,T/G, and $\mathrm{G} / \mathrm{G}$ ) could be distinguished by analysis of the chromatograms. The $T / T$ genotype would result in an F/F phenotype (low affinity), $T / G$ in a V/F phenotype, whereas $G / G$ would result in a $\mathrm{V} / \mathrm{V}$ phenotype (high affinity). We subsequently analysed a total of 76 samples for the V/F polymorphism and a total of 29 samples were found to be homozygous for $T(F / F), 42$ samples were heterozygous $(V / F)$, and 5 samples were homozygous for $\mathrm{G}(\mathrm{V} / \mathrm{V})$. Hence, the $\mathrm{T}$ allele was overall the most prevalent; $66 \% \mathrm{~T}$ compared to $34 \% \mathrm{G}$. To validate the results obtained from the sequencing, we used sequence-specific primers (SSPs) to specifically amplify the variants of the FCGR3A gene separately. For this validation, we selected a total of 11 samples. The gel electrophoresis results confirmed the sequencing results (Supplementary Fig. S1). Altogether, these data showed that the developed Sanger sequencing approach is reliable to identify polymorphisms in the FCGR3A gene. 
a
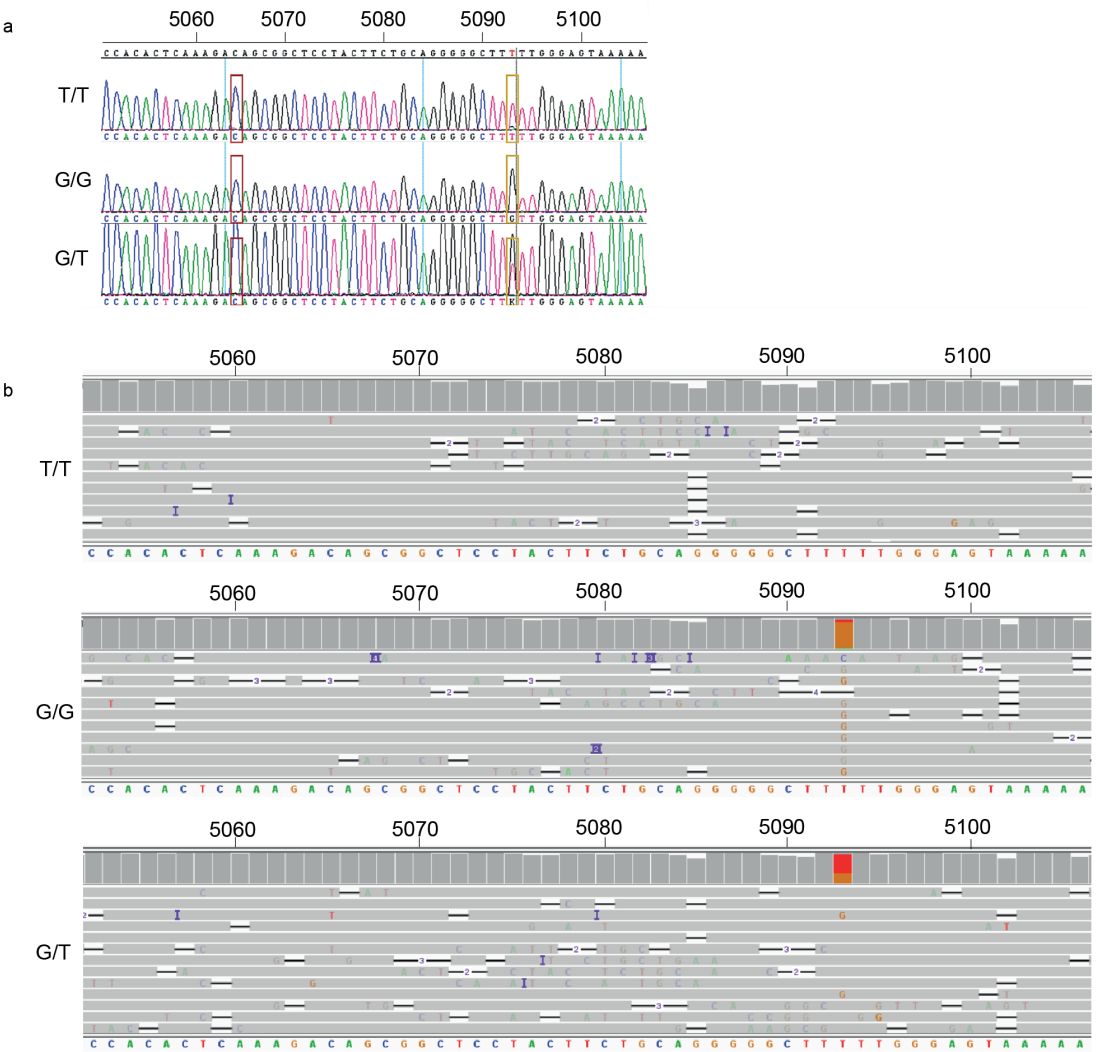

Figure 3. Detection of V158F polymorphism by showing 3 different genotypes, homozygous T, homozygous $\mathbf{G}$, and heterozygous. (a) Electropherograms show the Sanger-based sequencing result around the $\mathrm{V} 158 \mathrm{~F}$ polymorphism. The red square indicates nucleotide position 5064, which is used to check whether the FCGR3B gene is co-amplified. The yellow square indicates nucleotide position 5093, used for determining the V158F polymorphism. Nucleotide code K indicates that both $\mathrm{T}$ and $\mathrm{G}$ are present. (b) MinION sequencing result around the $\mathrm{V} 158 \mathrm{~F}$ polymorphism. Dark grey bars on the top show the sequence coverage identical to the consensus sequence. If the sequence is not identical to the consensus the bars will have the color of the corresponding nucleotide. The light grey lines show a small part of the reads obtained with the MinION run and the sequence at the bottom shows the consensus sequence. The first result represents a sample homozygous for $\mathrm{T}$ at position nucleotide 5093 (coverage: A: 0\%: C: 2\% G: 2\% T: 96\%), which was also used as consensus, the second sample is homozygous for G (coverage: A: $3 \%$ C: $2 \%$ G: $85 \%$ T: $9 \%$ ), and the third is heterozygous at nucleotide position 5093 (coverage: A: 1\% C: 3\% G: 32\% T: 64\%). 


\section{Detection of extended, full length polymorphisms is feasible using Sanger- and MinION Nanopore-based sequencing}

The results from the 1 KGP database analysis on FCGR3A gene polymorphisms revealed that there were more polymorphisms within the FCGR3A gene than previously described. Additionally, the 1KGP polymorphism frequency database showed that some of these polymorphisms occurred in the worldwide population with a frequency higher than $1.0 \%$. We therefore envisioned that detection of extended full length polymorphisms, including the non-coding regions, in this gene could facilitate future studies on the functional relevance of the FcyRIIla receptor polymorphism. To investigate the feasibility of detecting polymorphisms in the FCGR3A gene, we set up a pilot study and amplified the whole FCGR3A gene region for 14 DNA samples and subsequently sequenced using two approaches: Sanger- and MinION sequencing (Oxford Nanopore Technologies). Despite full length amplification, we did not perform full length sequencing for Sanger sequencing for this pilot and used primers covering a part of intron 3, exon 4, intron 4, and 3'UTR region. MinION is a novel portable real-time single molecule sequencing device developed to sequence long regions with ultra-long reads. With this technique, we were therefore able to sequence the complete full-length gene, also including all non-coding gene regions. MinION amplification primers were also tagged enabling us to barcode and sequence multiple samples simultaneously. After sequencing, we analysed the sequencing results and compared the results obtained by SBT with those obtained by MinION and with the data from the $1 \mathrm{KGP}$.

In this pilot study, we detected 23 SNPs in the FCGR3A gene of the 14 individuals (Table 3). Of these 23 SNPs, 13 were also identified by the $1 \mathrm{KGP}$ and two of these SNPs (G3121A and T3155C) were found with a MAF < 0.01. The only exonic SNP T5093G (V158F polymorphism) is listed in 1KGP database as "failed variant" and the allelic frequency data is not available in this database. We therefore used the MAF data from other databases (GO-ESP and ExAC) in table 3. The 10 SNPs not identified by the 1 KGP were located in the non-coding intron 3, intron 4 or 3'UTR region.

Of the 23 detected SNPs, nine were identified by both the Sanger and MinION technique. Since the other 14 SNPs were located in regions outside the Sanger sequence area, these were only identified by MinION. This result demonstrates that MinION sequencing can be used to determine full length FCGR3A polymorphism. Although the results of MinION sequencing were similar to Sanger sequencing, some caution should be taken when reading the sequencing results in the region where V158F polymorphism is located (Fig. 3b). We observed that MinION could misreport the presence of heterozygous $\mathrm{G} / \mathrm{T}$ where it would be reported as a homozygous $\mathrm{T} / \mathrm{T}$ genotype (depending on the analysis settings/percentage of nucleotides present), 
most likely due to the presence of homopolymer sequence within the region. This might actually be the reason why it is reported as a "failed variant" in the $1 \mathrm{KG}$ project, since all NGS methods encounter difficulties in analysing homopolymer regions. Altogether, we demonstrated that using full-length Sanger-based and MinION-based sequencing methods we could detect both known as well as new polymorphisms within the FCGR3A gene.

Table 3. SNPs found within the FCGR3A gene detected by SBT and MinION, compared to $1 \mathrm{KG}$.

\begin{tabular}{|c|c|c|c|c|c|c|c|c|}
\hline \multirow[b]{2}{*}{ SNP } & \multirow[b]{2}{*}{$\begin{array}{l}\text { Chromosomal } \\
\text { position }\end{array}$} & \multirow[b]{2}{*}{$\begin{array}{l}\text { Gene } \\
\text { position }\end{array}$} & \multicolumn{3}{|c|}{ Detected in } & \multirow[b]{2}{*}{ 1KG project } & \multirow[b]{2}{*}{ SNP name } & \multirow[b]{2}{*}{ MAF } \\
\hline & & & $\begin{array}{l}\text { Gene } \\
\text { location }\end{array}$ & SBT & MinION & & & \\
\hline $\mathrm{G} 224 \mathrm{~T}$ & 1:161519411 & 224 & Intron 1 & $\mathrm{No}^{*}$ & Yes & Yes & rs10917571 & 0,34 \\
\hline C516G & 1:161519119 & 516 & Intron 1 & $\mathrm{No}^{*}$ & Yes & Yes & rs4656317 & 0,26 \\
\hline $\mathrm{G} 727 \mathrm{~A}$ & 1:161518908 & 727 & Intron 1 & $\mathrm{No}^{*}$ & Yes & Yes & rs138032965 & 0,11 \\
\hline G1463A & 1:161518172 & 1463 & Intron 3 & $\mathrm{No}^{*}$ & Yes & No & $\mathrm{n} / \mathrm{a}$ & $\mathrm{n} / \mathrm{a}$ \\
\hline G1793C & 1:161517842 & 1793 & Intron 3 & $\mathrm{No}^{*}$ & Yes & No & $\mathrm{n} / \mathrm{a}$ & $\mathrm{n} / \mathrm{a}$ \\
\hline C2418T & 1:161517217 & 2418 & Intron 3 & $\mathrm{No}^{*}$ & Yes & Yes & rs4656312 & 0,13 \\
\hline A2967G & 1:161516668 & 2967 & Intron 3 & $\mathrm{No}^{*}$ & Yes & No & $\mathrm{n} / \mathrm{a}$ & $\mathrm{n} / \mathrm{a}$ \\
\hline G3121A & 1:161516514 & 3121 & Intron 3 & $\mathrm{No}^{*}$ & Yes & Yes & rs545876704 & $<0,01$ \\
\hline T3155C & 1:161516480 & 3155 & Intron 3 & $\mathrm{No}^{*}$ & Yes & Yes & rs180923798 & $<0,01$ \\
\hline A3187G & 1:161516448 & 3187 & Intron 3 & $\mathrm{No}^{*}$ & Yes & No & $\mathrm{n} / \mathrm{a}$ & $\mathrm{n} / \mathrm{a}$ \\
\hline G3624T & 1:161516011 & 3624 & Intron 3 & Yes & Yes & Yes & rs6672453 & 0,11 \\
\hline G3683T & 1:161515952 & 3683 & Intron 3 & Yes & Yes & Yes & rs7526944 & 0,41 \\
\hline G3763C & 1:161515872 & 3763 & Intron 3 & Yes & Yes & Yes & rs149210339 & 0,03 \\
\hline A4083G & $1: 161515552$ & 4083 & Intron 3 & Yes & Yes & No & $\mathrm{n} / \mathrm{a}$ & $\mathrm{n} / \mathrm{a}$ \\
\hline A4327C & 1:161515308 & 4327 & Intron 3 & Yes & Yes & No & $\mathrm{n} / \mathrm{a}$ & $\mathrm{n} / \mathrm{a}$ \\
\hline A4459G & 1:161515176 & 4459 & Intron 3 & Yes & Yes & Yes & rs10429882 & 0,41 \\
\hline T5093G & 1:161514542 & 5093 & Exon 4 & Yes & Yes & Yes & rs396991 & $\begin{array}{l}0.27^{* *}- \\
0.33^{* * *}\end{array}$ \\
\hline T5728C & 1:161513907 & 5728 & Intron 4 & Yes & Yes & No & $\mathrm{n} / \mathrm{a}$ & $\mathrm{n} / \mathrm{a}$ \\
\hline C5876G & 1:161513759 & 5876 & Intron 4 & $\mathrm{No}^{*}$ & Yes & No & $\mathrm{n} / \mathrm{a}$ & $\mathrm{n} / \mathrm{a}$ \\
\hline T6187C & 1:161513448 & 6187 & Intron 4 & $\mathrm{No}^{*}$ & Yes & No & $\mathrm{n} / \mathrm{a}$ & $\mathrm{n} / \mathrm{a}$ \\
\hline T6258G & 1:161513377 & 6258 & Intron 4 & $\mathrm{No}^{*}$ & Yes & Yes & rs426615 & 0.46 \\
\hline C6904T & 1:161512731 & 6904 & 3'UTR & Yes & Yes & Yes & rs7539036 & 0.11 \\
\hline G8054C & 1:161511581 & 8054 & 3'UTR & $\mathrm{No}^{*}$ & Yes & No & $\mathrm{n} / \mathrm{a}$ & $\mathrm{n} / \mathrm{a}$ \\
\hline
\end{tabular}

Using the same amplification primers, 14 DNA samples were sequenced using SBT and MinION. MAF represents the Minor Allele Frequency data from 2504 individuals obtained from the1KG database except for rs396991 (F158V) were the MAF was obtained from the GO-ESP** and ExAC*** database. No* $=$ this position was not included within the SBT sequence region. 


\section{DISCUSSION}

NK cells are the principal mediator of ADCC due to the high expression of the activating FcyRIIla and the absence of the inhibitory FcyRIIlb on their surface [3]. The large availability of clinical grade antibodies triggering ADCC against cancer cells has put increased focus on NK cell-mediated ADCC and emphasizes the relevance of the FcyRIIla for cancer immunotherapy [4]. In addition, a few studies underlined the functional relevance of FcyRIIla in the transplantation setting by showing that NK cell mediated ADCC could play a role in allograft rejection [33]. Albeit several FCGR3A gene polymorphisms have been shown to impact NK cell mediated ADCC, full length gene polymorphism has not been determined. Hence, we provide here an overview of FCGR3A gene polymorphisms, as well as two improved sequencing methods for further gene exploration.

In this study, with 234 polymorphisms identified, we demonstrated that FCGR3A gene is more polymorphic than currently known; 34 SNPs were located in the exons and only 3 of them had a MAF $>0.01$. We identified two non-synonymous SNPs either by Sanger sequencing/MinION sequencing or in 1KGP. The first one was rs10127939, representing the FcyRIIIA-48L/R/H previously shown to influence ADCC $[22,23]$. We did not detect this polymorphism in our full-length sequencing samples presumably because of our limited sample size and the fact that the frequency of this polymorphism is relatively low in the population (MAF $=0.039$ and 0.027 ). The second non-synonymous SNP in the coding region was rs396991, representing the V158F polymorphism which we detected both by Sanger- and by MinION sequencing. In our test panel the V/F phenotype (G/T genotype) is the most common (55\%) followed by $F / F$ (T/T genotype, 38\%) and V/V (G/G genotype, 7\%). The presence of $\mathrm{V} 158 \mathrm{~F}$ polymorphism has been previously investigated in individuals from different populations, including ethnic groups from Singapore [34], the Netherlands, Great Britain, Norway [35] and Japan [36]. Overall these studies reported the V/F or F/F phenotype as the most frequent, whereas the V/V was the least frequent phenotype in all populations, which is comparable to our results and could suggest some kind of selective pressure on FCGR3A. Our study set up did not allow us to reliably compare V158F gene- and allele frequencies between the 34 samples from the Guadaloupe population vs the 42 samples from our institute or with the results from the 1 KGP. The major reason for this was the lack of information on the exact ethnic background of the individuals and the low sample size. Given the known highly heterogeneous background of the Guadaloupe population, it would, however, be highly interesting to compare this population with other populations in a future study. 
In this study, we demonstrated that the majority of FCGR3A gene polymorphism is located in the non-coding regions and at least 33 of the 200 identified non-coding SNPs have a MAF>0.01 in the 2504 individuals of the $1 \mathrm{KG}$ project. As introns have been demonstrated to be involved in gene regulation [37] and many intronic polymorphisms could exhibit functional significance [38] it might be worthwhile to perform additional functional studies. SNPs located in the intron regions could potentially affect RNA splicing by altering the sequences of the $5^{\prime} / 3^{\prime}$ splice site, branch point, polypyrimidine tract or intronic splicing enhancer/silencer motifs. A study on FCGR2C gene interestingly showed that a mutation in an intronic splice site introduced novel stop codons resulting in a loss of FcyRllc expression [39]. In this study we have investigated the two consensus splice site sequences on the $5^{\prime}$ and $3^{\prime}$ end of the intron (GT on $5^{\prime}$ and AG on $3^{\prime}$ ) and we already observed one SNP (rs544630563) at the 3' of intron 4, turning AG into GG, although it was found with low frequency in the $1 \mathrm{KGP}$ database (MAF <0.01). Additionally, as in a recent paper reviewing different studies on different disease genes, several mutations deep within the introns (for example 100 base pairs upstream exon-intron boundary) were identified as being associated to multiple diseases [40]. In line with our data, where we observed intronic polymorphisms located upstream the exon-intron boundary, it would be interesting to look at the association of these polymorphisms with the functionality of the FcyRIIla receptor.

In the present study, we successfully set up a Sanger- and a MinION-based protocol to sequence the FCGR3A gene. We subsequently demonstrated that both Sanger sequencing and MinION were able to identify FCGR3A polymorphisms present in the 1KGP database. While Sanger sequencing is based on the capillary electrophoresis, MinION technology consists of nanopores embedded in an electrically resistant membrane through which a current is applied, causing a potential which flows through the aperture of the nanopores. The changes observed in the current correspond with 5 to 6 nucleotides passing through the nanopores. This electrical signal is translated into reads that can be analysed and by this technology, MinION can sequence reads up to hundreds of kilo base pairs. For both techniques, we performed identical full length amplification of the gene. However, MinION has the advantage of directly generating full length gene reads and phasing of the two variants is possible without group-specific amplification. Although MinION allows full-gene sequencing of various samples in a relatively short time, this technology is not yet widely implemented. Compared to conventional sequencing approaches MinION has a lower accuracy and sensitivity and therefore more reads must be generated. We demonstrated a good concordance between Sanger sequencing and MinION and were able to identify the V158F polymorphism in all samples using both MinION and Sanger. Nonetheless, a homopolymer region, such as the sequence around $\mathrm{V} 158 \mathrm{~F}$, is known to 
be problematic in all next generation sequencing approaches, apparently including MinION and presumably this also explains the lack of data for this region in the $1 \mathrm{KGP}$. The challenge of analyzing such homopolymer regions with MinION sequencing has been described in several other studies as well [41-43]. Hence despite its usefulness for full length gene analysis, Sanger sequencing for now seems the preferred method when only analysis of $\mathrm{V} 158 \mathrm{~F}$ polymorphism is required.

In summary, we showed that FCGR3A gene is highly polymorphic especially in the noncoding regions of the gene requiring functional studies to investigate the functional consequences. Additionally, we demonstrated that our Sanger- and MinION-based sequencing approaches can be used to identify the extended polymorphisms of the gene. Although further optimization and validation is warranted, we also identified MinION as a powerful method to perform direct full-length FCGR3A gene sequencing. 


\section{MATERIAL AND METHODS}

\section{Subjects}

FCGR3A sequences were studied in a test panel consisting of 76 distinct samples with unknown DNA sequence, 42 of them were volunteers from the institute and 36 were individuals from the Guadeloupe islands [44]. Samples were left over from diagnostic procedures which does not require ethical approval in the Netherlands under the Dutch Code for Proper Secondary Use of Human.

\section{DNA isolation}

Genomic DNA was extracted from ethylenediamine tetraacetic acid (EDTA) blood samples using the QIAamp DNA blood mini kit (Qiagen, Hilden, Germany). DNA concentrations were measured using the NanoDrop ND-1000 spectrophotometer (Thermo Scientific, Wilmington, Delaware).

\section{Amplification of the FCGR3A gene for SBT and MinION sequencing Amplification primers}

Primers specific for the FCGR3A gene were designed by comparing the sequences of the FCGR3A and FCGR3B genes, including their polymorphisms, and finding the discrepancies among them. Due to the extreme homology of the genes, some generic primers (not specific for the FCGR3A gene) were also designed as a control and always used in combination with a specific primer.

\section{Polymerase Chain Reaction}

The entire FCGR3A gene, including the 5'UTR and $3^{\prime} U T R$, was amplified using an FCGR3A gene-specific forward primer and a generic reverse primer, producing a 9654 bases long polymerase chain reaction (PCR) product. The PCR reaction contained 300 ng of genomic DNA, 67 mM Tris-HCl (pH 8.8) (Merck, Darmstadt, Germany), $16.6 \mathrm{mM}$ ammonium sulfate (Merck), $0.01 \%$ Tween 20 (Merck), $1.5 \mathrm{mM} \mathrm{MgCl}$ (Life Technologies, Austin, Texas), $0.2 \mathrm{mM}$ of each dNTP (GE Healthcare, Diegem, Belgium), $0.1 \mu \mathrm{g} / \mu \mathrm{l} \mathrm{cresol}$ red (Sigma-Aldrich, St. Louis, Missouri), 5\% glycerol (Alfa Aesar, Karlsruhe, Germany), 15 pmol of each primer (Sigma-Aldrich) and $2.5 \mathrm{U}$ of Expand Long Template PCR System (Roche, Basel, Switzerland) with a final volume of $30 \mu \mathrm{l}$. The PCR program consisted of an initial denaturation step of 2 minutes at $94^{\circ} \mathrm{C}$; followed by 10 cycles of 15 seconds at $94^{\circ} \mathrm{C}, 30$ seconds at $63^{\circ} \mathrm{C}$ and 4 minutes at $68^{\circ} \mathrm{C}$; then 10 cycles of 15 seconds at $94^{\circ} \mathrm{C}, 30$ seconds at $60^{\circ} \mathrm{C}$ and 6 minutes at $68^{\circ} \mathrm{C}$; afterwards 10 cycles of 15 seconds at $94^{\circ} \mathrm{C}, 30$ seconds at $60^{\circ} \mathrm{C}$ and 10 minutes at $68^{\circ} \mathrm{C}$; and a final elongation step of 7 minutes at $68^{\circ} \mathrm{C}$. The PCR products were checked by electrophoresis using a $1.5 \%$ agarose gel containing $0.5 \mu \mathrm{g} / \mu \mathrm{l}$ ethidium bromide (Sigma-Aldrich). 


\section{MinION amplification}

The same amplification primers used for SBT were used for the MinION sequencing mixture, with a tag-sequence (indicated as italic and red) added to the ends to enable barcoding amplification for identification of different samples after all samples were pooled.

\section{Sanger sequencing of $\mathrm{V} 158 \mathrm{~F}$ region}

Amplicons were purified by ExoSAP-IT (Affymetrix, Santa Clara, California) following the manufacturer's protocol. Purified amplicons were sequenced using $A B I$ BigDye Terminator Chemistry (Life Technologies) and an ABI 3730 sequencer (Life Technologies) with a forward and a reverse sequencing primer.

The sequencing mixture consisted of $1 \mu \mathrm{l}$ purified PCR product, $0.5 \mu \mathrm{l}$ sequencing primer (5 pmol, Sigma-Aldrich), $1 \mu \mathrm{l}$ of BigDye Terminator v1.1 mix, $1.5 \mu \mathrm{l} 5 \mathrm{x}$ Big Dye Terminator sequencing buffer and $6 \mu \mathrm{l}$ distilled water. The PCR program consisted of: 1 minute at $95^{\circ} \mathrm{C}$, followed by 25 cycles of 10 seconds at $95^{\circ} \mathrm{C}, 5$ seconds at $50^{\circ} \mathrm{C}$, and 4 minutes at $60^{\circ} \mathrm{C}$. Successively, the mixtures were purified by Sephadex G-50 Fine (GE Healthcare Life Sciences, Little Chalfont, UK) and placed in the ABI 3730 sequencer for capillary electrophoresis sequencing. The chromatograms were aligned with a reference sequence obtained from the $1 \mathrm{KG}$ project and analysed using DNASTAR Lasergene SeqMan Pro (DNASTAR Lasergene, Madison, Wisconsin).

\section{FCGR3A gene sequencing using Sanger Sequencing}

For FCGR3A gene sequencing using SBT, several sequencing primers were used to cover different locations in the gene.

\section{MinION Nanopore-based sequencing}

Amplicons were barcoded and sequenced following Oxford Nanopore's instructions (NSK-LSK208). In short, we purified the amplicons using CleanPCR beads (GC Biotech, Alphen aan den Rijn, the Netherlands) followed by determining DNA concentration using a DS-11 spectrophotometer (DeNovix, Delaware, USA). Next, $48 \mathrm{ng}$ of amplicon was barcoded using the PCR barcoding Kit 1 (Oxford Nanopore Technologies, Oxford, UK) and LongAmp Taq 2x (New England Biolabs, Massachusetts, USA) followed by purification of the barcoded PCR product using CleanPCR beads and determination of DNA concentration. The barcoded DNA samples were pooled to an end volume of $1 \mu \mathrm{g}$ in $45 \mu \mathrm{l}$ and an endrepair/dA-tailing was performed (NEBNext Ultra II End-Repair/dAtailing module, New England Biolabs) followed by a purification step using AMPure XP beads (Beckman Coulter, California, USA). After that, DNA adapter ligation was performed using NEB Blunt/TA ligase master mix (New England Biolabs) and samples 
were purified using MyOne C1 Dynabeads (Thermo Fisher Scientific, Massachusetts, USA). Of this adapter library, $75 \mu \mathrm{l}$ was loaded into a FLO-MIN106 flow cell. Sequencing run was performed and base calling was done using Albacore software (V1.2.4, Oxford Nanopore Technologies). Sequencing data was analysed using in-house software and Integrative Genomics Viewer (IGV) [45].

\section{PCR amplification using sequence specific primers for SBT validation}

The sequence specific primers (SSPs) consisted of one primer specific for the T allele and one for the G allele. An FCGR3A gene-specific primer was used in combination with the SSPs to assure specific amplification of the FCGR3A gene. The PCR program was almost identical to the SBT amplification protocol described in this article, except that the annealing temperature used for SSP PCR was $63^{\circ} \mathrm{C}$ during the first 10 cycles.

\section{Genome Project Data Analysis}

Based on the publicly available data present in the third phase of the $1 \mathrm{KG}$ project (http://phase3browser.1000genomes.org/index.html), including 2504 individuals originating from 26 different populations, the FCGR3A gene comprised 8259 bp located on chromosome 1: 161511549-161519818 (reverse direction). This sequence corresponds to the FCGR3A-001 protein coding transcript and the start of exon 1 (position 1:161519634) was used as nucleotide position 1 in this paper. We recorded the polymorphism and its location on the chromosome as well as the gene location (position of nucleotide and region (i.e. UTR, exon, or intron)). The population genetic tool was used to acquire an overview of the overall allele frequencies and the frequencies within different population. 


\section{List of primers}

\begin{tabular}{|l|l|l|}
\hline \multicolumn{2}{|l|}{ SBT Amplification Primers } & \multicolumn{2}{l}{} \\
\hline Direction & Sequence $\left(5^{\prime}\right.$ to $\left.{ }^{\prime}\right)$ & Location 1000 Genomes \\
\hline FW & GCTGCCTGGGTTCATTTCCA & $1: 161520918-161520938$ \\
\hline RV & CCTCTGCCCAGGCCTCTA & $1: 161511283-161511301$ \\
\hline
\end{tabular}

\begin{tabular}{|l|l|l|}
\hline \multicolumn{2}{|l|}{ MinI0N Amplification Primers } & \multicolumn{2}{l}{} \\
\hline Direction & Sequence $\left(5^{\prime}\right.$ 'to $\left.3^{\prime}\right)$ & Location 1000 Genomes \\
\hline FW & TTTCTGTTGGTGCTGATATTGCGCTGCCTGGGTTCATTTCCA & $1: 161520918-161520938$ \\
\hline RV & ACTTGCCTGTCGCTCTATCTTCCCTCTGCCCAGGCCTCTA & $1: 161511283-161511301$ \\
\hline
\end{tabular}

\begin{tabular}{|l|l|l|}
\hline \multicolumn{2}{|l|}{ SBT V158F region Sequencing Primers } & \multicolumn{2}{l}{} \\
\hline Direction & Sequence $\left(5^{\prime}\right.$ to $\left.3^{\prime}\right)$ & Location 1000 Genomes \\
\hline FW & GTGTTCAAGGAGGAGACC & $1: 161514701-1: 161514719$ \\
\hline RV & ACTCAACTTCCCAGTGTGATT & $1: 161514701-1: 161514719$ \\
\hline
\end{tabular}

\begin{tabular}{|l|l|l|l|}
\hline \multicolumn{2}{|l|}{ SBT FCGR3A gene Sequencing Primers } & \multicolumn{2}{l}{} \\
\hline Direction & Sequence $\left(5^{\prime}\right.$ to $\left.3^{\prime}\right)$ & Location 1000 Genomes & Specificity \\
\hline FW & CTAATAATGATTCATCTCTYTGC & $1: 161525783-1: 161525805$ & Intron 3 \\
\hline FW & TGCTKAAAAAGTAAGTGGWTAG & $1: 161525803-1: 161525824$ & Intron 3 \\
\hline RV & GGTAAGTATTATAATGGCAYAAG & $1: 161526243-1: 161526260$ & Intron 3 \\
\hline RV & TTATAGGTAAGTATTATAATGGC & $1: 161526248-1: 161526265$ & Intron 3 \\
\hline FW & KTTTGGCAGTGYCAACCWTC & $1: 161528867-1: 161528886$ & Exon 5 / 3'UTR \\
\hline FW & TCCACCTGGGTACCAAGTC & $1: 161528898-1: 161528916$ & Exon 5 / 3'UTR \\
\hline RV & TTCTATGTTTCCTGCTGCTTG & $1: 161529146-1: 161529166$ & Exon 5 / 3'UTR \\
\hline RV & RGGATCTGGCTCTGAGTTC & $1: 161529163-1: 161529182$ & Exon 5 / 3'UTR \\
\hline FW & GTGTTCAAGGAGGAAGACC & $1: 161514701-1: 161514719$ & V158F region \\
\hline RV & ACTCAACTTCCCAGTGTGATT & $1: 161514701-1: 161514719$ & V158F region \\
\hline
\end{tabular}

\begin{tabular}{|l|l|l|l|}
\hline \multicolumn{2}{|l|}{ SSP Amplification Primers } & \multicolumn{2}{l|}{} \\
\hline Direction & Sequence $\left(5^{\prime}\right.$ to $\left.3^{\prime}\right)$ & Location 1000 Genomes & Specificity \\
\hline RV & AAGACACATTTTTACTCCCAAA & $1: 161514521-1: 161514542$ & T allele \\
\hline RV & AAGACACATTTTTACTCCCAAC & $1: 161514521-1: 161514542$ & G allele \\
\hline FW & GCTGCCTGGGTTCATTTCCA & $1: 161520918-161520938$ & FCGR3A \\
\hline
\end{tabular}




\section{DATA AVAILABILITY}

All data generated or analysed during this study are included in this published article (and its Supplementary Information file).

\section{AUTHOR CONTRIBUTIONS}

N.M.M., T.I.O, co-wrote the main manuscript text. L.W. supervised the writing of the manuscript. I.O.R, S.J.J.M, were responsible for the practical part and data acquisition. N.M.M generated the figures. M.G. were responsible for the bio-informatics part of the project. N.M.M. and T.I.O. were responsible for data analysis and interpretation of the data. C.E.M.V and L.W. supervised the interpretation of the data. Critical reviews were given by G.M.J.B, M.G.J.T, C.E.M.V, and L.W. Final approval was given by all authors.

\section{COMPETING INTERESTS}

The author(s) declare no competing interests. 


\section{REFERENCES}

1. Vivier E, Tomasello E, Baratin M, et al (2008) Functions of natural killer cells. Nat Immunol 9:503510. doi: $10.1038 /$ ni1582

2. Campbell KS, Hasegawa J (2013) Natural killer cell biology: An update and future directions. J Allergy Clin Immunol 132:536-544. doi: 10.1016/j.jaci.2013.07.006

3. Seidel UJE, Schlegel P, Lang P (2013) Natural Killer Cell Mediated Antibody-Dependent Cellular Cytotoxicity in Tumor Immunotherapy with Therapeutic Antibodies. Front Immunol 4:76. doi: 10.3389/fimmu.2013.00076

4. Wang W, Erbe AK, Hank JA, et al (2015) NK Cell-Mediated Antibody-Dependent Cellular Cytotoxicity in Cancer Immunotherapy. Front Immunol 6:368. doi: 10.3389/fimmu.2015.00368

5. Bowles JA, Weiner GJ (2005) CD16 polymorphisms and NK activation induced by monoclonal antibody-coated target cells. J Immunol Methods 304:88-99. doi: 10.1016/j.jim.2005.06.018

6. Koene HR, Kleijer M, Algra J, et al (1997) Fc gammaRIlla-158V/F polymorphism influences the binding of IgG by natural killer cell Fc gammaRIIla, independently of the Fc gammaRIIla-48L/R/H phenotype. Blood 90:1109-14.

7. Wu J, Edberg JC, Redecha PB, et al (1997) A novel polymorphism of FcyRIlla (CD16) alters receptor function and predisposes to autoimmune disease. J Clin Invest 100:1059-1070. doi: 10.1172/JCI119616

8. Congy-Jolivet N, Bolzec A, Ternant D, et al (2008) Fc gamma Rllla expression is not increased on natural killer cells expressing the Fc gamma RIlla-158V allotype. Cancer Res 68:976-80. doi: 10.1158/0008-5472.CAN-07-6523

9. Weng W-K, Levy R (2003) Two immunoglobulin G fragment C receptor polymorphisms independently predict response to rituximab in patients with follicular lymphoma. J Clin Oncol 21:3940-7. doi: 10.1200/JCO.2003.05.013

10. Cartron G, Dacheux L, Salles G, et al (2002) Therapeutic activity of humanized anti-CD20 monoclonal antibody and polymorphism in IgG Fc receptor FcgammaRIlla gene. Blood 99:754758. doi: 10.1182/blood.V99.3.754

11. Musolino A, Naldi N, Bortesi B, et al (2008) Immunoglobulin G fragment C receptor polymorphisms and clinical efficacy of trastuzumab-based therapy in patients with HER-2/neupositive metastatic breast cancer. J Clin Oncol 26:1789-96. doi: 10.1200/JCO.2007.14.8957

12. Bibeau F, Lopez-Crapez E, Di Fiore F, et al (2009) Impact of FcyRlla-FcyRIlla Polymorphisms and KRAS Mutations on the Clinical Outcome of Patients With Metastatic Colorectal Cancer Treated With Cetuximab Plus Irinotecan. J Clin Oncol 27:1122-1129. doi: 10.1200/JCO.2008.18.0463

13. Taylor RJ, Chan S-L, Wood A, et al (2009) FcyRllla polymorphisms and cetuximab induced cytotoxicity in squamous cell carcinoma of the head and neck. Cancer Immunol Immunother 58:997-1006. doi: 10.1007/s00262-008-0613-3

14. Hatjiharissi E, Xu L, Santos DD, et al (2012) Increased natural killer cell expression of CD16, augmented binding and ADCC activity to rituximab among individuals expressing the FC $\gamma$ Brief report Increased natural killer cell expression of CD16, augmented binding and ADCC activity to rituximab amon. Blood J 110:2561-2564. doi: 10.1182/blood-2007-01-070656

15. Tamura K, Shimizu C, Hojo T, et al (2011) Fc R2A and 3A polymorphisms predict clinical outcome of trastuzumab in both neoadjuvant and metastatic settings in patients with HER2-positive breast cancer. Ann Oncol 22:1302-1307. doi: 10.1093/annonc/mdq585

16. Botticelli A, Mazzuca F, Borro M, et al (2015) FCGRs Polymorphisms and Response to Trastuzumab in Patients With HER2-Positive Breast Cancer: Far From Predictive Value? World J Oncol 6:437440. doi: 10.14740/wjon934w 
17. Hidalgo LG, Sis B, Sellares J, et al (2010) NK Cell Transcripts and NK Cells in Kidney Biopsies from Patients with Donor-Specific Antibodies: Evidence for NK Cell Involvement in AntibodyMediated Rejection. Am J Transplant 10:1812-1822. doi: 10.1111/j.1600-6143.2010.03201.x

18. Venner JM, Hidalgo LG, Famulski KS, et al (2015) The molecular landscape of antibody-mediated kidney transplant rejection: Evidence for NK involvement through CD16a Fc receptors. Am J Transplant 15:1336-1348. doi: 10.1111/ajt.13115

19. Paul P, Picard C, Sampol E, et al (2018) Genetic and Functional Profiling of CD16-Dependent Natural Killer Activation Identifies Patients at Higher Risk of Cardiac Allograft Vasculopathy. Circulation 137:1049-1059. doi: 10.1161/CIRCULATIONAHA.117.030435

20. Takami a, Espinoza JL, Onizuka M, et al (2011) A single-nucleotide polymorphism of the Fcy receptor type IIIA gene in the recipient predicts transplant outcomes after HLA fully matched unrelated BMT for myeloid malignancies. Bone Marrow Transplant 46:238-43. doi: 10.1038/ bmt.2010.88

21. Shimizu S, Tanaka Y, Tazawa H, et al (2016) Fc-Gamma Receptor Polymorphisms Predispose Patients to Infectious Complications After Liver Transplantation. Am J Transplant 16:625-633. doi: 10.1111/ajt.13492

22. de Haas M, Koene HR, Kleijer M, et al (1996) A triallelic Fc gamma receptor type IIIA polymorphism influences the binding of human IgG by NK cell Fc gamma RIlla. J Immunol 156:2948-55.

23. Dong C, Ptacek TS, Redden DT, et al (2014) Fcy Receptor Illa Single-Nucleotide Polymorphisms and Haplotypes Affect Human IgG Binding and Are Associated With Lupus Nephritis in African Americans. Arthritis Rheumatol 66:1291-1299. doi: 10.1002/art.38337

24. Grier JT, Forbes LR, Monaco-Shawver L, et al (2012) Human immunodeficiency-causing mutation defines CD16 in spontaneous NK cell cytotoxicity. J Clin Invest 122:3769-3780. doi: 10.1172/ $\mathrm{JCl} 64837$

25. Oboshi W, Watanabe T, Yukimasa N, et al (2016) SNPs rs4656317 and rs 12071048 located within an enhancer in FCGR3A are in strong linkage disequilibrium with rs396991 and influence NK cell-mediated ADCC by transcriptional regulation. Hum Immunol 77:997-1003. doi: 10.1016/j. humimm.2016.06.012

26. Lassauniere R, Shalekoff S, Tiemessen CT (2013) A novel FCGR3A intragenic haplotype is associated with increased FcgammaRIIla/CD16a cell surface density and population differences. Hum Immunol 74:627-634. doi: 10.1016/j.humimm.2013.01.020

27. Chen S, Wen X, Li J, et al (2017) Association of FCGR2A/FCGR3A variant rs2099684 with Takayasu arteritis in the Han Chinese population. Oncotarget 8:17239-17245. doi: 10.18632/ oncotarget. 12738

28. Qin F, Wang H, Song L, et al (2016) Single nucleotide polymorphism rs 10919543 in FCGR2A/ FCGR3A region confers susceptibility to takayasu arteritis in chinese population. Chin Med $J$ (Engl) 129:854-859. doi: 10.4103/0366-6999.178965

29. Chai L, Song Y-Q, Zee K-Y, Leung WK (2010) SNPs of Fc-gamma receptor genes and chronic periodontitis. J Dent Res 89:705-10. doi: 10.1177/0022034510365444

30. Ravetch J V, Perussia B (1989) Alternative membrane forms of Fc gamma RIII(CD16) on human natural killer cells and neutrophils. Cell type-specific expression of two genes that differ in single nucleotide substitutions. J Exp Med 170:481-97.

31. Dall'Ozzo S ébastien, Andres C, Bardos P, et al (2003) Rapid single-step FCGR3A genotyping based on SYBR Green I fluorescence in real-time multiplex allele-specific PCR. J Immunol Methods 277:185-192. doi: 10.1016/S0022-1759(03)00123-6

32. Rosales $C$ (2017) Fcy receptor heterogeneity in leukocyte functional responses. Front Immunol 8:1-13. doi: 10.3389/fimmu.2017.00280

33. Valenzuela NM, Hickey MJ, Reed EF (2016) Antibody subclass repertoire and graft outcome following solid organ transplantation. Front Immunol. doi: 10.3389/fimmu.2016.00433 
34. Chong KT, Ho WF, Koo SH, et al (2007) Distribution of the FcgammaRIlla $176 \mathrm{~F} / \mathrm{V}$ polymorphism amongst healthy Chinese, Malays and Asian Indians in Singapore. Br J Clin Pharmacol 63:32832. doi: $10.1111 / j .1365-2125.2006 .02771 . x$

35. Van Sorge NM, Van Der Pol WL, Jansen MD, et al (2005) Severity of Guillain-Barré syndrome is associated with Fcy Receptor III polymorphisms. J Neuroimmunol 162:157-164. doi: 10.1016/j. jneuroim.2005.01.016

36. Van Der Pol WL, Jansen MD, Sluiter WJ, et al (2003) Evidence for non-random distribution of Fcy receptor genotype combinations. Immunogenetics 55:240-246. doi: 10.1007/s00251-0030574-9

37. Chorev M, Carmel L (2012) The Function of Introns. Front Genet 3:55. doi: 10.3389/ fgene.2012.00055

38. Cooper DN (2010) Functional intronic polymorphisms: Buried treasure awaiting discovery within our genes. Hum Genomics 4:284. doi: 10.1186/1479-7364-4-5-284

39. Nagelkerke SQ, Kuijpers TW (2015) Immunomodulation by IVIg and the role of Fc-gamma receptors: Classic mechanisms of action after all? Front Immunol. doi: 10.3389/fimmu.2014.00674

40. Vaz-Drago R, Custódio N, Carmo-Fonseca M (2017) Deep intronic mutations and human disease. Hum Genet 136:1093-1111. doi: 10.1007/s00439-017-1809-4

41. Ip CLC, Loose M, Tyson JR, et al (2015) MinION Analysis and Reference Consortium: Phase 1 data release and analysis. F1000Research. doi: 10.12688/f1000research.7201.1

42. Lu H, Giordano F, Ning Z (2016) Oxford Nanopore MinION Sequencing and Genome Assembly. Genomics, Proteomics Bioinforma 14:265-279. doi: 10.1016/j.gpb.2016.05.004

43. Cornelis S, Gansemans Y, Deleye L, et al (2017) Forensic SNP Genotyping using Nanopore MinION Sequencing. Sci Rep 7:41759. doi: 10.1038/srep41759

44. Voorter CEM, Groeneweg M, Joannis MO, et al (2014) Allele and haplotype frequencies of HLADPA1 and -DPB1 in the population of Guadeloupe. Tissue Antigens 83:147-153. doi: 10.1111/ $\tan .12271$

45. Robinson JT, Thorvaldsdóttir H, Winckler W, et al (2011) Integrative genomics viewer. Nat Biotechnol 29:24-6. doi: 10.1038/nbt.1754 


\section{SUPPLEMENTARY FIGURE}

a

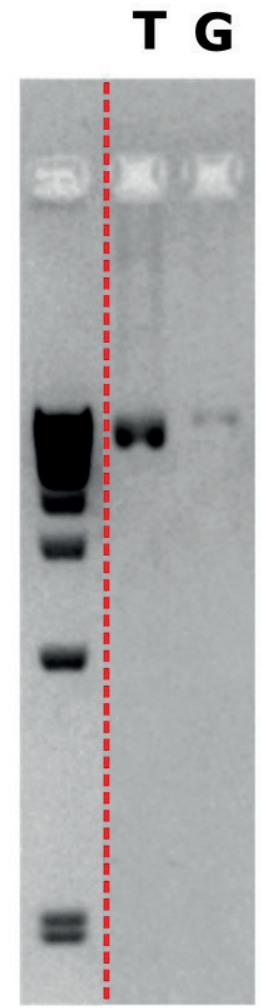

b

$\mathbf{T} \mathbf{G}$

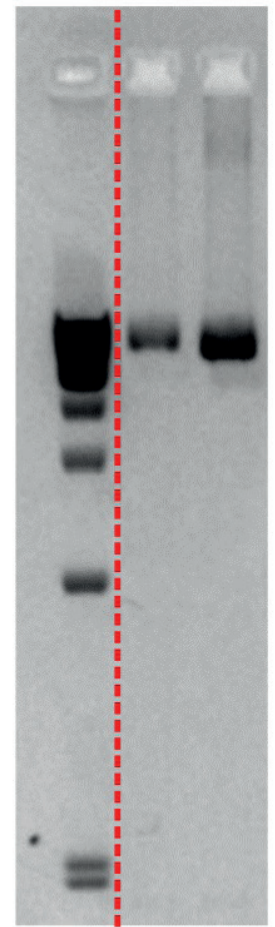

\section{C}

T G

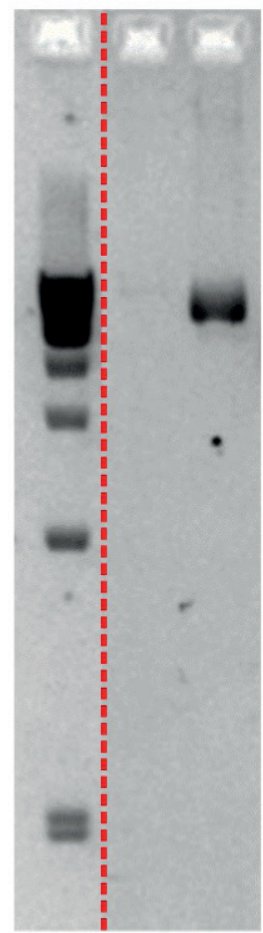

Supplementary Figure S1. The detection of V/F polymorphism by SSP showing 3 different genotypes. Three different DNA samples representing homozygous T (a), heterozygous (b), and homozygous G (c) genotype. T on the lane means the SSP used for amplification was specific for the T allele, while a $\mathrm{G}$ indicates the SSP used for amplification was specific for the $\mathrm{G}$ allele. Each figure was derived from 3 different gels. Red dotted lines marked the border between the ladder and the samples. 
88

Chapter 4 


\section{Clinical and immunological significance of HLA-E in stem cell transplantation and cancer}

Lotte Wieten1, Niken M. Mahaweni1,2, Christina E.M. Voorter1, Gerard M.J. Bos2, Marcel G.J. Tilanus1

1 Department of Transplantation Immunology, Maastricht University Medical Center+, Maastricht, The Netherlands.

2 Department of Internal Medicine, Division of Hematology, Maastricht University Medical Center+, Maastricht, The Netherlands

Tissue Antigens. 2014 Dec;84(6):523-35. doi: 10.1111/tan.12478. Review. 


\section{ABSTRACT}

Human leukocyte antigen-E (HLA-E) is a non-classical HLA class I molecule that canonically binds peptides derived from the leader sequence of classical HLA class I. HLA-E can also bind peptides from stress protein [e.g. heat shock protein 60 (Hsp60)] and pathogens, illustrating the importance of HLA-E for anti-viral and anti-tumor immunity. Like classical HLA class I molecules, HLA-E is ubiquitously expressed, however, it is characterized by only a very limited sequence variability and two dominant protein forms have been described (HLA-E*01:01 and HLA-E*01:03). HLA-E influences both the innate and the adaptive arms of the immune system by the engagement of inhibitory (e.g. NKG2A) and activating receptors [e.g. a $\beta$ T cell receptor ( $a \beta T C R$ ) or NKG2C] on NK cells and CD8 T cells. The effects of HLA-E on the cellular immune response are therefore complex and not completely understood yet. Here, we aim to provide an overview of the immunological and clinical relevance of HLA-E and HLA-E polymorphism in stem cell transplantation and in cancer. We review novel insights in the mechanism via which HLA-E expression levels are controlled and how the cellular immune response in transplantation and cancer is influenced by HLA-E. 


\section{INTRODUCTION}

Major histocompatibility complex (MHC) class I molecules have an essential function in both the innate and the adaptive immune system through the presentation of peptides from intracellular proteins to lymphocytes and by acting as ligands for NK cell receptors. MHC class I molecules can be divided in classical MHC class I molecules (MHC class la) and non-classical MHC class I molecules (MHC class Ib). Human leucocyte antigen-E (HLA-E) is a member of the group of non-classical MHC class I molecules also including HLA-G and HLA-F. Non classical MHC molecules have been especially recognized for their immunomodulatory role. Expression of HLA-G and $-F$ is mainly restricted to specific tissues, e.g. the placenta. In contrast, expression of HLA-E is more ubiquitous and virtually every healthy cell in the body positive for HLA class I also expresses HLA-E. The molecular structure of HLA-E closely resembles that of the classical MHC class I molecules (i.e. HLA-A, $-B$ and $-C$ ) but there are some obvious differences; HLA-E displays limited polymorphism as compared to the highly polymorphic HLA class I molecules, and thus far two dominant protein variants have been recognized (1). In addition, the peptide binding cleft of HLA-E allows binding of only a restricted set of peptides while classical HLA class I molecules bind a wide variety of peptides. HLA-E interacts with inhibitory and activating receptors present on NK cells and T cells, hence, having a dual function in the immune system $(2,3)$. HLA-E has been shown to bind pathogen-derived peptides, to act as an antigen provoking an immune response in the transplantation setting and it can be aberrantly expressed by tumor cells. However, the exact influence of HLA-E on anti-viral- or antitumor immunity and transplantation outcome is complex and not completely known.

\section{Structural characteristics of HLA-E}

HLA-E is expressed by virtually every healthy cell in the body but the expression levels of HLA-E are relatively low compared to class I. The molecular structure of HLA-E closely resembles that of HLA class I and consists of a heavy chain made up, by the extracellular a1-3 domains, a transmembrane region and the intracellular domains of the protein. Equally to the classical HLA class I molecules, the heavy chain of the HLA-E molecule pairs with an invariant light chain i.e. $\beta 2$-microglobulin, $\beta 2-m$ (1). The $a 1$ and $a 2$ domains form the peptide binding cleft of the molecule consisting of eight $\beta$ folds at the bottom of the groove and two flanking $\alpha$-helices. For stable cell surface expression, peptide binding is required and HLA-E typically binds short (8-10 amino acid) peptides. Classical HLA class I peptide clefts usually have two anchor residues and one or more secondary residues for fine tuning between allotypes whereas the HLA-E peptide cleft comprises 5 of these anchor residues ( $p 2,3,6,7$ and 9 ) that in combination with its limited polymorphism result in the binding of a much 
more restricted peptide repertoire $(1,4)$. This is an illustration of the more conserved nature of HLA-E and presumably also of the different function of HLA-E in the immune system. Under homeostatic conditions, HLA-E binds peptides from intracellular proteins and primarily peptides derived from the leader sequences of classical HLA class I molecules. These leader sequences become available when they are cut from the rest of the HLA molecule by signal peptidases in the endoplasmic reticulum (ER) during translocation of the HLA molecule to the cell surface (5). However, HLA-E has also been shown to bind a peptide derived from heat shock protein 60 ( $\mathrm{Hsp60}$ ), a protein that is abundantly expressed by cells exposed to a wide variety of stress factors (6). In addition, HLA-E can bind peptides from intracellular pathogens, e.g. from cytomegalovirus (CMV) (UL40), Hepatitis C, Epstein-barrvirus (EBV), human immunodeficiency virus (HIV), mycobacteria, Salmonella (GroEL). These pathogenderived peptide sequences can be different from the canonical class I leader peptide sequences. More details on structural characteristics of HLA-E and an overview of HLA-E binding peptides, including their sequences are reviewed elsewhere $(1,2,7)$.

\section{Regulation of HLA-E expression}

HLA-E expression levels are predominantly controlled through HLA-E binding peptides. Availability of HLA-E binding peptides is important, but, the exact sequence of the peptide is also relevant as it has been shown to determine peptide binding affinity. Hence, peptides binding with a lower affinity result in less stable HLA-E molecules on the cell surface and thus lower expression levels as compared with peptides binding HLA-E with a high affinity (8). Through the availability of class I leader peptides, HLA-E expression levels are directly linked to expression levels of classical HLA class I molecules. Viral infection can reduce the availability of leader peptides either through direct inhibition of HLA expression or by interfering with the antigen presentation machinery (e.g. interference with transporter associated with antigen processing (TAP)) leading to the down regulation of HLA class I (9). The reduction in HLA class I facilitates the escape of virally infected- or tumor cells from immune surveillance by cytotoxic CD8 T cells (10). Through the limited availability of class I leader peptides, HLA-E expression shall go down as well rendering virally infected cells more susceptible for killing by NK cells. Several viruses encode (TAP independent) HLA-E binding peptides ensuring that, even in the absence of HLA class I leader peptides, HLA-E expression levels remain high enough to inhibit NKG2A expressing NK cells. This has been extensively studied for CMV which encodes a number of HLA-E binding peptides, amongst them the gpUL40-derived VMAPRTLVL and VMAPRTLIL peptides, having the same sequence as some of the HLA class I leader peptides, but not requiring TAP functioning (9). Interestingly, a recent study has demonstrated that in the absence of a functional TAP, HLA-E presents a different, 
TAP independent peptide repertoire consisting of 500 peptides that do not have sequence overlap with the TAP dependent repertoire (11). The close resemblance of the TAP independent HLA-E repertoire with that of HLA-A2 suggests that in a TAP deficient situation, HLA-E has the same function as classical HLA class I in the activation of a CD8 T cell response. Under homeostatic conditions, HLA-E expression levels are controlled mainly through the binding of HLA class I leader peptides. In virally infected cells, however, the HLA-E system is hijacked by the virus to guarantee a level of HLA-E surface expression mimicking that of normal healthy cells to facilitate the escape from immunesurveillance.

Recently microRNA's have been described as peptide independent regulatory mechanism of HLA-E expression. MicroRNA's are small non coding RNA's that interfere with RNA translation by binding to the $3^{\prime} U T R$ of the gene which leads to direct inhibition of translation or to degradation of the mRNA also resulting in reduced levels of HLA-E protein. Nachmani et al, identified miR-376a as a microRNA that can bind to the 3'UTR of HLA-E (12). In addition, they showed that editing of miR-376a, by CMV encoded ADAR1, reduces the binding of this microRNA leading to enhanced expression of HLA-E. This study is illustrative for the potential role of posttranscriptional control of HLA expression. MicroRNA control of gene expression has been reported for HLA-G (13) and classical HLA class I (14) as well. Moreover various viruses have been shown to encode microRNA's or microRNA editing proteins like ADAR1, and availability of microRNA's can be tissue dependent which could be an explanation for tissue restricted HLA expression (12). 


\section{Functional relevance of HLA-E}

The effect of HLA-E and alterations in expression levels of HLA-E on the cellular immune response is complex because HLA-E interacts with both activating and inhibitory receptors on NK cells and CD8 T cells and, depending on the receptor and the responding cell, engagement of HLA-E can lead to immune activation or suppression (summarized in Figure 1).

An important receptor family interacting with HLA-E is the family of CD94/NKG2, C-type lectin-like receptors, expressed on NK cells. NKG2 family members can trigger inhibition or activation of target cell lysis by NK cells ((15) and reviewed in Ref.7). NKG2A and NKG2C are two of several members of the NKG2 family which associate with CD94 as heterodimer. NKG2A has immunoreceptor tyrosine-based inhibition motif (ITIM) in its cytoplasmic tail while NKG2C can bind to DAP12 protein bearing immunoreceptor tyrosine-based activation motif (ITAM). Therefore, binding of HLA-E and CD94/NKG2A provides an inhibitory signal to the NK cell, whereas interaction of HLA-E and CD94/NKG2C delivers an activation signal to the NK cell (Figure 1). The two receptors have been demonstrated to recognize overlapping epitopes of HLA-E and competitively bind to HLA-E with NKG2A having higher affinity compared to NKG2C (16-18). Thus, the interaction between NK cells and HLA-E predominantly results in NK cells inhibition.

But what could be the functional relevance of this mechanism for the immune system? HLA-E is expressed by nearly all healthy cells and tissues. NK cell activation is determined by the signaling balance between inhibitory and activating receptors. Engagement of HLA with inhibitory receptors like killer immunoglobuline like receptors (KIRs for HLA-ABC) and NKG2A (for HLA-E) protects healthy cells from killing by NK cells (19). Activating NK cell ligands are frequently stress- or pathogenassociated proteins. In a situation where HLA is downregulated (e.g. upon viral infection or neoplastic transformation), inhibitory signaling via HLA will be reduced and if a potential target cell expresses sufficient levels of activating NK cell ligands, NK cell will kill the target cell via the release of cytotoxic granules or death receptors (20). However, several pathogens, amongst them CMV, have been shown to encode HLA-E binding peptides, e.g. CMV encoded gpUL40-derived peptides VMAPRTLVL and VMAPRTLIL that mimic the leader peptides from HLA-A and $C$ resulting in upregulation of HLA-E and protection of the infected cells from NK cell attack and illustrates the importance of HLA-E in the pathogenesis of viral disease (9). 


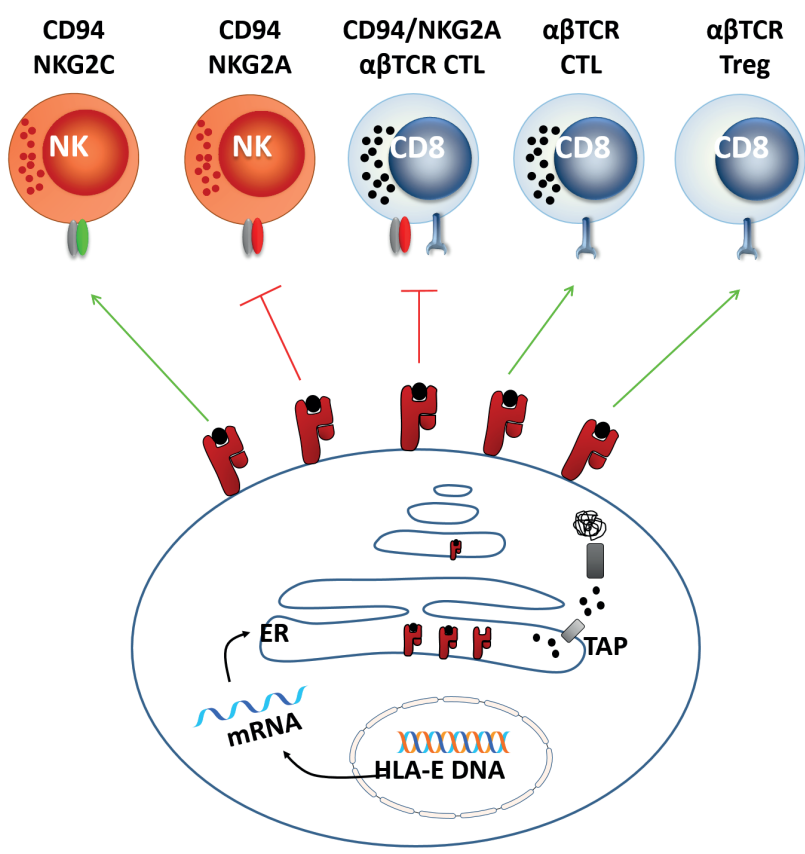

Figure 1. The activating and inhibitory effect of human leukocyte antigen-E (HLA-E) on NK cell and T cell subsets. HLA-E is stably expressed on the cell surface after the binding of HLA class I leader peptides or peptides derived from intracellular proteins degraded by the proteasome and transported into the endoplasmic reticulum (ER) via TAP. Cell surface HLA-E peptide complexes can have an inhibitory (red lines) or activating (green arrows) effect on NK cells or CD8 T cells. Upon activation, NK cells and cytotoxic CD8 T cells (CTL) will mainly have cytotoxic- and IFNY-producing function contributing to anti-viral, anti-tumor and alloreactive immune-responses. Regulatory $\mathrm{T}$ cells will contribute to the suppression of other immune cells.

The affinity of HLA-E interaction with NKG2 receptors is influenced by the peptide bound to the HLA-E molecule, and for example HLA-E in complex with leader peptide from HLA-CW7 (VMAPRALLL) results in reduced affinity of HLA-E for CD94/NKG2A $(3,17,18)$. Moreover, we and others showed that HLA-E presenting the HLA-G derived peptide has superior capacity to activate NKG2C expressing NK cells as compared to other leader peptides (Lauterbach et al submitted, (21). During cellular stress, Hsp60 competes with other HLA class I molecules to bind with HLA-E. However, because the HLA-E:Hsp60 complex cannot bind to CD94/NKG2A, it results in a reduction of inhibition of NK cell's cytotoxicity therefore making the cell more vulnerable to NK cell killing (6). The HCVcore $_{35-44}$ peptide (YLLPRRGPRL) has also been shown to stabilize HLA-E on the cell surface without inhibition of NKG2A positive NK cells whereas it synergistically enhanced the inhibitory effects of HLA class I leader peptides (22). The NKG2A coreceptor CD94 can also occur as homodimer and the authors proposed that the HLA-E:HCV-core ${ }_{35-44}$ complex engages CD94 homodimers but not CD94:NKG2A heterodimers. Although 
these CD94 homodimers cannot signal themselves, their clustering probably stabilizes the immunological synapse which augments inhibitory signaling via CD94:NKG2A and represents another way for viruses to enhance inhibition of NKG2A positive NK cells (23).

A subset of peripheral blood T cells expresses NK receptor (NKR) such as CD94/NKG2 enabling these $T$ cells, mostly $C D 45 R^{+} C D 8^{+} T$ cells to recognize HLA-E (figure 1) (24). The effect of CD8 T cells NKR engagement with Qa-1 (mouse homology of HLA-E molecule) peptide complex is determined by the NKG2 subunit in the same way as for NK cells; The interaction between Qa-1 peptide complex with CD94/NKG2A receptor on CD8 T cells conveys an inhibitory signal for the CD8 T cells (25) while binding of Qa-1 peptide complex with CD94/NKG2C expressed on CD8 T cells results in CD8 T cell activation and cytotoxic function (26). In addition to this recognition pathway, García et al and Pietra et al demonstrated that HLA-E restricted cytotoxic CD8 T cells could also interact with HLA-E peptide complex via their a $\beta$ T cell receptor (TCR) (figure 1) (27-29). The differences between the recognition via NKR and TCR lies in the outcome of the T cells response towards target cells and priming requirement. Because HLA-E has been shown to present a broad range of pathogen derived peptide HLA-E restricted CD8 T cells could play an important role in the clearance of pathogens especially in a situation where classical HLA class I is downregulated, as reviewed in Refs. 2 and 28. Unlike the NKR pathway, this pathway requires priming of CD8 T cells. A well characterized example is the recognition, by CD8 aßTCR of CMV-derived UL40 peptide presented by HLA-E in individuals expressing HLA-C alleles that do not have the same leader peptide sequence as the UL40 peptide (i.e. HLA-Cw2 -Cw7, -Cw15 and-Cw18), or CD8 T cells specific for Mycobacterium tuberculosis and Salmonella typhi that have been isolated and could lyse infected target cells in an HLA-E restricted manner $(30,31)$. In addition to cytotoxic effects, HLA-E:TCR interaction has been shown to trigger $T$ cells having a more regulatory function; TCR engagement with target cells expressing Qa-1 peptide complex results in Qa-1 restricted CD8 $T$ cells suppression of autoreactive CD4+ T cells (32). Jiang et al demonstrated that these regulatory mechanisms also apply in humans illustrating that HLA-E restricted CD8 $T$ cells serve as a regulatory system in the peripheral immune system to maintain selftolerance by discriminating self from non-self (33).

\section{HLA-E polymorphism}

The HLA-E locus is located, together with the classical HLA class I and II genes, within the MHC region on the short arm of chromosome 6. The HLA-E gene contains eight exons encoding the leader peptide (exon 1), the extracellular a1-3 domains (exon 2-4), the transmembrane region (exon 5) and the intracellular domains (exon 6 and 7 ) of the heavy chain of the HLA-E molecule (1). The HLA-E stop codon (TAA) is present in exon 7. Despite similarities in protein structure, HLA-E molecules are far less polymorphic 
than classical HLA class I molecules and until now only 15 alleles have been assigned encoding six protein variants (HLA-E*01:01, ${ }^{*} 01: 03,{ }^{*} 01: 04,{ }^{*} 01: 05,{ }^{*} 01: 06$ and $\left.{ }^{*} 01: 07\right)$ as a result of non-synonymous mutations (IMGT/HLA database version 3.17.0). Multiple alleles encode the two most frequent phenotypes; HLA-E*01:01 (encoded by the alleles $E^{*} 01: 01: 01: 01, E^{*} 01: 01: 01: 02, E^{*} 01: 01: 01: 03, E^{*} 01: 01: 02$ ) and HLA-E*01:03 (encoded by the alleles $E^{*} 01: 03: 01: 01, E^{*} 01: 03: 01: 02, E^{*} 01: 03: 02: 01, E^{*} 01: 03: 02: 02, E^{*} 01: 03: 03, E^{*} 01: 03: 04$, $E^{*}$ 01:03:05). Frequencies of HLA-E*01:03 were shown to be higher than HLA-E*01:01 in two southern Han populations (34), a Japanese population (35) and American-Indians from Columbia (36). Nevertheless in most other worldwide populations, HLA-E*01:01 and $H L A-E^{*} 01: 03$ could be detected at equal frequencies suggesting some form of balancing selection and a functional difference between the two alleles $(37,38)$. The two protein variants differ by a single amino acid difference at codon 107 of the a 2 domain of the HLA-E heavy chain (i.e. arginine in HLA-E*01:01 and Igycine in HLA-E*01:03; Figure 2 panel A). This substitution has very limited structural consequences except for the presence of an additional hydrogen bond in the HLA-E*01:01 molecule involving the p107 side chain (8). Nevertheless, cell surface expression of HLA-E*01:03 is higher than that of $H L A-E^{*} 01: 01$ which has been demonstrated to be the consequence of the slightly higher peptide-binding affinity of HLA-E*01:03 as compared to HLA-E*01:01 (8).

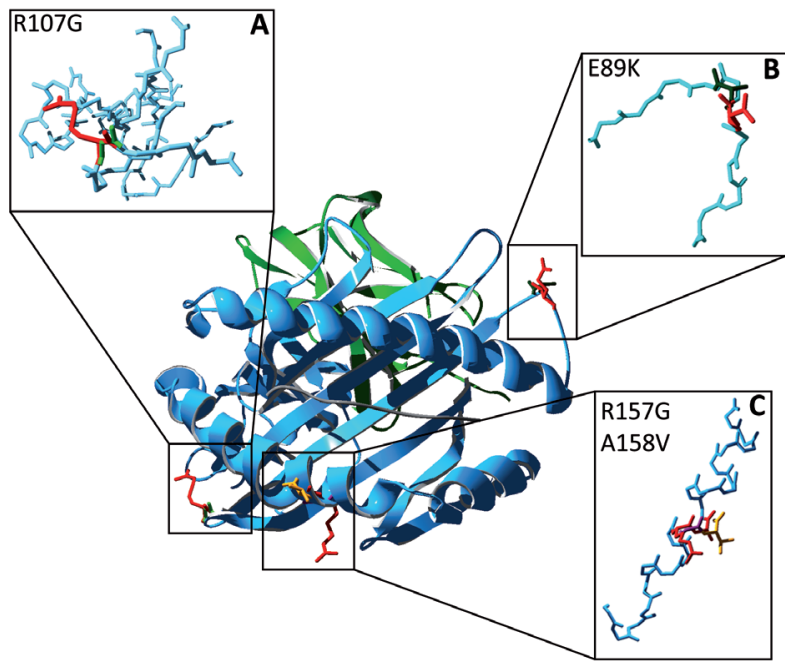

Figure 2. Alignment of HLA-E*01:01, *01:03, *01:04, *01:05, *01:06 and *01:07. The blue ribbons represent human leukocyte antigen-E (HLA-E) and the green ribbon $\beta 2$ microglobulin. (A) Red denotes the arginine (R) on position 107, present in HLA-E*01:01 and *01:07; green denotes the glycine (G) present in HLA-E*01:03, * 01:04, ${ }^{*} 01: 05$ and *01:06. (B) Dark green denotes the lysine $(\mathrm{K})$ present in HLA-E*01:05 on position 89, red the glutamic acid (E) present in all other alleles. (C) Purple denotes the glycine $(G)$ present in $H L A-E^{*}$ 01:04 on position 157, red the arginine present in all other alleles. Yellow denotes the valine (V) present in $\mathrm{HLA}-\mathrm{E}^{*} 01: 07$ on position 158, red denotes the alanine (A) present in all other alleles. The serine (S) at position 267 of HLA-E*01:06 (P267S) is not visible in this view, as it is in the a3 domain. Models were obtained from the PDB database (http://www.rcsb.org, accession numbers $1 \mathrm{KTL}$ and $1 \mathrm{MHE})(4,8,68)$ or modeled using SWISS-MODEL (http://swissmodel.expasy.org/) (69-71) and visualized using Swiss PdbViewer (72) and POV-Ray for Windows (Persistence of Vision Pty. Ltd.,Williamstown, Victoria, Australia. http://www.povray.org/). 
The existence of $H L A-E^{*} 01: 04$, still remains questionable. Like $H L A-E^{*} 01: 03$, HLA-E*01:04 encodes a glycine at codon 107. In contrast to the other protein variants, having an arginine, HLA-E*01:04 has a glycine at position 157 (Figure 2 panel C). However, upon its detection in a Japanese population, the presence of this allele could not be confirmed in any other population (including Japanese) or study suggesting that it might represent a sequencing artefact (35). Recently, high resolution sequencing techniques have been exploited to study HLA-E nucleotide variability in more detail which resulted in the identification of three new nonsynonymous alleles; HLA-E*01:05, HLA-E*01:06 and HLA-E*01:07. HLA-E*01:05 encodes a lysine at position 89 while all the other alleles have a glutamic acid at that position (Figure 2 panel B). HLA-E*01:06 differs from the other alleles by the presence of a serine at position 267, instead of a proline, which is located in the a3 domain of the protein. HLA-E*01:07 uniquely has a valine at position 158, all the other alleles have an alanine (Figure 2 panel C). Like HLA-E*01:01, HLA-E*01:07 expresses an arginine at position 107 whereas HLA-E*01:04, HLA-E*01:05, HLA-E*01:06 shares with HLA-E*01:03 a glycine on position 107 (Figure 2 panel $A$ ). The frequencies of the new alleles $\left(E^{*} 01: 05, E^{*} 01: 06\right.$ and $\left.E^{*} 01: 07\right)$ can be defined once larger population studies using high resolution typing are available to confirm whether these alleles are indeed relatively rare as compared to the HLA-E*01:01 and HLA-E*01:03 variants.

The difference in cell surface expression of $H L A-E^{*} 01: 01$ vs $H L A-E^{*} 01: 03$ illustrates that variation in the coding region of the gene can have a functional impact. Polymorphism in the regulatory regions of the gene, for example the promoter region or the $3^{\prime} U T R$, could have an additional quantitative or qualitative influence on HLA expression. For HLA-C for example, it has been shown that a single nucleotide polymorphism (SNP) in the 3'UTR abrogates the binding of hsa-miR-148 microRNA which allows HLA-C alleles expressing this SNP to escape from post-translational control resulting in higher cell surface expression (14). Variation in $3^{\prime} U T R$ for HLA-E remains virtually unexplored but a recent study analyzed genetic variation in the coding region (exons 1-4, including introns) and in the 3'UTR region in 104 Brazilian samples and in 14 different populations of the 1000genomes project (phase 1, 1092 individuals) (39). Analyzing this database revealed the presence of 15 SNP variations in the coding region and 13 in the $3^{\prime}$ UTR. These variable sites could be arranged in 33 haplotypes, 29 of them encoding HLA-E*01:01 or HLA-E*01:03 proteins, and were present in an overall frequency of 0.982 in all populations studied, strengthening the idea that these variants are the most frequently occurring ones. In the same study, additional linkage disequilibrium (LD) evaluation uncovered a strong LD between the two most frequent polymorphic positions, genomic position +424 (synonymous substitution in exon 2) and +756 (the Gly/Arg substitution in HLA-E*01:01 vs 
HLA-E*01:03) but not between the 3'UTR and the coding sequence, which could be indicative of a recombination hotspot between the coding region and the $3^{\prime} U T R$. We recently used the 1000genomes data including additional SNP databases (ESP, dbSNP, Uniprot and HapMap) to study nucleotide variability in the HLA-E gene and identified 7 synonymous and 30 non-synonymous SNPs in the coding region of HLA-E (exons 1-7), 48 SNPs in the introns and 32 SNPs in the untranslated regions that where not yet assigned in the IMGT/HLA database (Olieslagers et al in preparation). In addition, we used a full length sequencing approach (5'UTR to $3^{\prime}$ UTR) and identified a new intron variant and a new null allele that were both not identified in the 1000 genomes project. These high resolution studies emphasize that, HLA-E is more polymorphic than initially thought, both in the coding and in the regulatory regions. Nonetheless, it still remains a highly conserved gene as compared to the classical HLA class I alleles.

\section{HLA-E in allogeneic hematopoietic stem cell transplantation}

Allogeneic stem cell transplantation (allo-SCT) is an important treatment option for patients suffering from hematological malignancies. Depending on the underlying disease, allo-SCT can be an effective and curative treatment option, but severe complications can occur. Important complications after allo-SCT are graft versus host disease (GvHD) caused by donor-derived $\mathrm{T}$ cell reactivity against patient cells especially in the gut and the skin; Host versus Graft (HvG) responses, due to patient T cells attacking the graft leading to non-engraftment; disease relapse, presumably the result of residual tumor cells escaping donor NK- and T cell immunity; transplantation related mortalities (TRM) due to a cause that is unrelated to the underlying disease e.g. infection. HLA is an important determinant for the outcome of allo-SCT because of its high polymorphic nature and important function in antigen presentation. To avoid complications, high resolution typing is applied to, preferably completely, match for HLA-A, -B, -C, -DR and -DQ . HLA-E is not considered in the current matching criteria but its relevance for transplantation has been recognized; In mice, skin grafts from mice transgenically expressing HLA-E*01:03 were rejected by CD8 T cells from non-transgenic mice (40). Furthermore, in mixed lymphocyte reactions (MLR), HLA-E could trigger proliferation of human TCRaß alloreactive CD8 T cells having the capacity to kill target cells expressing HLA-E in complex with HLA class I leader- or viral peptides (41). In addition to the activation of alloreactive T cells, HLA-E can regulate NK cells expressing NKG2 receptor variants.

Because HLA-E*01:03 has been shown to be expressed higher on the cell surface than HLA-E*01:01 molecules and this could have an impact on the cellular immune response upon transplantation, a thus far limited number of studies addressed the influence of these genotypes on transplantation outcome (summarized in Table 1). Based on the two most frequent HLA-E alleles three possible genotypes exist; 


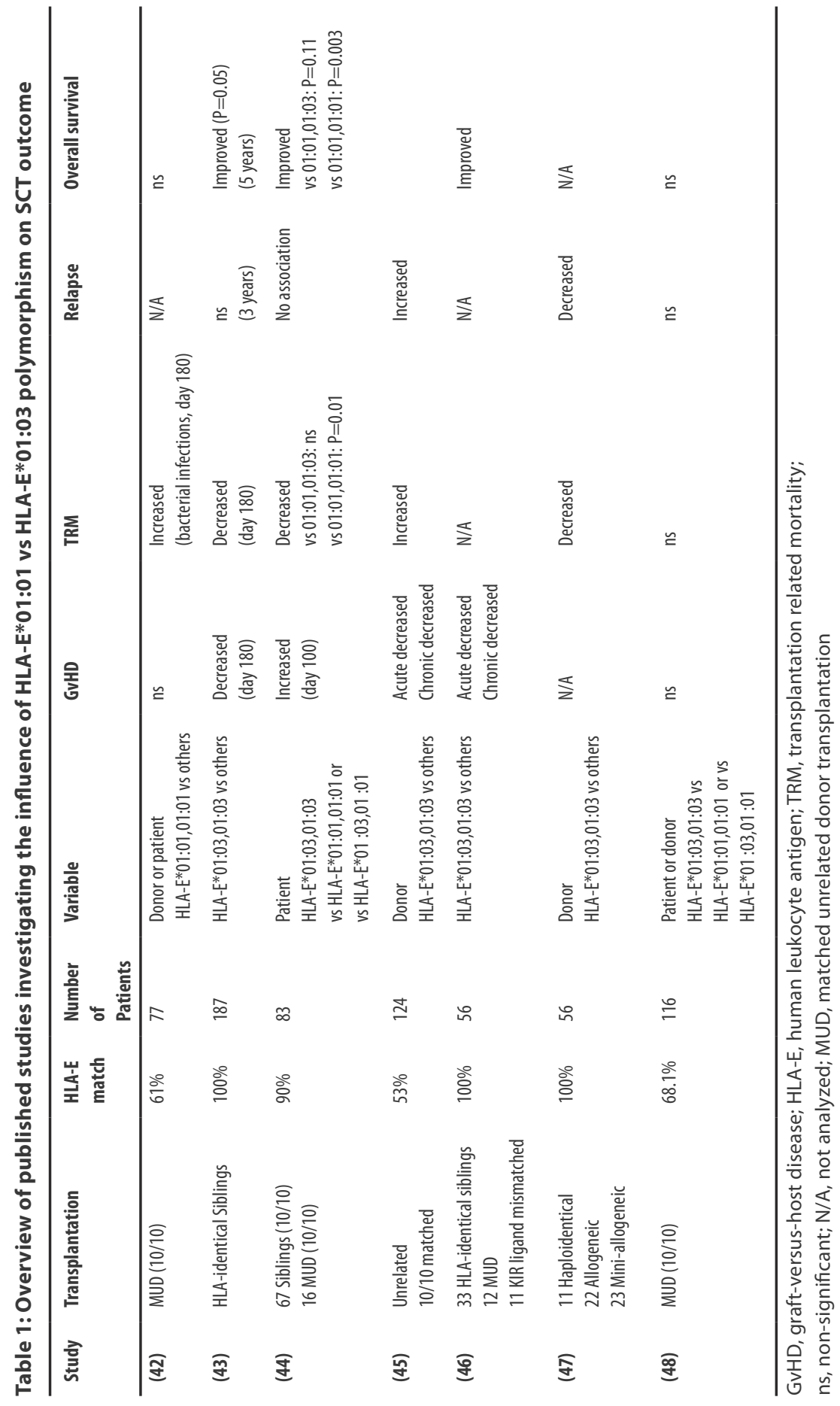


HLA-E*01:01,01:01, HLA-E*01:01,01:03 and HLA-E*01:03,01:03. In a first study with 77 unrelated donor-recipient pairs (10/10 matched), HLA-E*01:01,01:01, either in the donor or in the patient, was identified as a risk factor for the occurrence of severe bacterial infections but not for viral or fungal infections nor for acute GvHD (42). In a second study, with 187 HLA-identical (including HLA-E) sibling pairs, the same authors report an association between the HLA-E*01:03,01:03 genotype and a lower incidence of acute GVHD and TRM as well as a trend towards association of this genotype with improved survival (43). However, in contrast to the first study, no association was observed between HLA-E*01:01 homozygosity and the occurrence of severe infections (including bacterial), presumably because in this cohort only 5 of 187 patients experienced bacterial infections. Danzer et al analyzed 83 patients undergoing HLA-matched allo-SCT of either an unrelated- or a sibling donor and found that HLA-E*01:03 homozygous patients had a higher overall- and disease free survival and a decreased incidence of TRM when compared with HLA-E*01:01 homozygous patients (44). Of note, in comparison with HLA-E*01:03,01:01 heterozygous patients the survival difference did not reach significance. Furthermore, the cumulative incidence of relapse was comparable for all genotypes. In another study with a cohort of 124 allo-SCT of patients with unrelated donor pairs, the presence of HLA-E*01:03 alleles in the donor was associated with a lower risk of developing GVHD and a higher incidence of TRM and relapse (45). More recently, the influence of HLA-E*01:03 homozygosity versus the other 2 genotypes was studied in 56 HLA-E matched patients undergoing SCT with either an HLA-identical sibling-, MUD or KIR-ligand mismatched donor (46). This yielded no significant difference between the HLA-E*01:03,01:01 heterozygous and HLA-E*01:01 homozygous groups for any of the parameters. However, HLA-E*01:03 homozygosity was associated with a lower frequency of acute- and chronic GvHD and with improved survival. The same authors describe in a second study a lower incidence of relapse and improved disease free survival in $H L A-E^{*} 01: 03,01: 03$ patients as compared with patients having either one of the other two genotypes (47). Thus far only one published study showed that the HLA-E genotype did not have any influence on allo-SCT outcome (48). This study also determined whether the HLA-E matching status was associated with any of the above mentioned parameters but also this was not significant. Because the other association studies were either completely matched for HLA-E or did not report on the influence of HLA-E matching status, further studies in (partially) HLA-E mismatched cohorts are warranted to conclude whether matching status of HLA-E has an influence. Differences in clinical protocols, underlying disease and/or stem cell source might explain some of the discrepancies between the studies. Yet, in at least half of these studies the presence of HLA-E*01:03 alleles was associated with lower risk for TRM $(42-44,47)$ or $\operatorname{GvHD}(43,45,46)$ and an increased overall survival $(43$, 
$45,46)$ suggestive of a protective role of HLA-E*01:03 in allo-SCT. To translate these findings into clinical practice would require conformational studies in larger cohorts. Moreover, the above mentioned studies did not take the newly identified HLA-E alleles into account. Because the effect of the non-synonymous SNP discriminating HLA-E*01:06 from HLA-E*01:03 on functionality of the molecule is not known it would be relevant to address the contribution of HLA-E*01:06 in groups originally types as HLA-E*01:03.

The exact mechanism by which HLA-E influences transplantation outcome remains unknown. Depending on the cell type and the receptor, HLA-E engagement can lead to immune activation or inhibition and several mechanisms for this can be proposed; First, the direct activation of an HLA-E restricted/specific CD8 T cell response contributing to the clearance of pathogens and tumors but also to unwanted GvH tumor responses reviewed in (28). A higher cell surface expression of HLA-E, as observed for $H L A-E^{*} 01: 03$, could therefore lead to more efficient CD8 T cell reactivity which would be in line with the lower incidence of infection observed in HLA-E*01:03 homozygous patients $(42,43)$. A second mechanism is immunomodulation, HLA-E has been recognized for its immunoregulatory role in the placenta and HLA-E restricted CD8 Tregs exist. Higher expression levels of HLA-E*01:03 might therefore improve transplant tolerance via the activation of HLA-E specific Tregs. Though this has not been demonstrated experimentally yet, activation of Tregs and immunosuppression by HLA-E might explain the association between homozygosity HLA-E*01:03 allele and lower incidence of $\operatorname{GvHD}(43,45,46)$. As a third mechanism, HLA-E can inhibit NK cells and CD8 T cells expressing NKG2A thereby prohibiting immune effector cell responses against virally infected- or tumor cells. On the other hand, engagement of HLA-E with the activating NKG2C receptor on NK cells and T cells can provide immune activating responses such as the production of IFNy and cytotoxicity. NKG2C positive NK cells might have a unique role in anti-viral immunity as NK cells expressing CD94/ NKG2C have been shown to expand in response to CMV infected fibroblasts (26) and upon CMV reactivation upon stemcelltransplantation (49). These expanded NK cells exhibited memory like-features and were potent producers of IFN- $\gamma$ production upon reactivation. Although it is currently not clear whether HLA-E:NKG2C interaction is driving expansion of NKG2C positive NK cells, it is tempting to speculate that HLA-E presenting viral peptides or possibly TAP independent peptides contributes to the generation of a pool of "memory like" or "long-lived" effector NK cells with a potent anti-viral capacity. As NK cells are the first cells to come up after SCT, these subsets can be of particular importance in the first period after SCT. Finally, HLA-E could act via bystander cells e.g. endothelial cells that, upon activation, have been shown to attract and activate recipient T cells thus contributing to the graft rejection (50). In 
vitro, endothelial cells have been shown to express enhanced levels of HLA-E on the cell surface upon culture with pro-inflammatory cytokines (i.e. TNFa, IL-1 $\beta$ and IFNy) which protects them from NK cell mediated killing. In addition, they secrete soluble HLA-E which protects bystander cells (51). Also, HLA-E expressing endothelial cells could trigger HLA-E restricted CD8 T cells contributing to allograft rejection. Further functional studies will be helpful to unravel the complex influence of HLA-E on the cellular immune response upon transplantation.

\section{HLA-E in cancer}

Under the influence of the tumor environment, the process of metastasis and of the immune system, tumor cells can acquire or loose specific characteristics enabling them to survive in a hostile environment. This Darwinian selection of tumor cells is called cancer immunoediting, and because CD8 T cells and NK cells control tumor growth they have an important influence on the development of immune-escape tumor variants (52). Immunogenicity of tumors, especially for T cells, is largely depending on the presence of HLA molecules, presenting tumor-associated antigens, on the cell surface of tumor cells and dendritic cells. From a T cell perspective it is therefore not surprising that tumor cells frequently display reduced or complete lack of classical HLA class I expression because this enables them to escape from CD8 T cell killing $(10,53)$. On the other hand, tumor cells lacking cell surface expression of HLA class I become targets for NK cells (missing-self hypothesis) if they co-express activating ligands for the NK cell (20).

HLA-E expression mainly depends on the availability of HLA class I leader peptides, a reduction in expression of HLA class I normally results in a lower expression of HLA-E. In tumors however, this relation can be disturbed (54) and HLA-E expression by tumor cells, even in the complete absence of classical HLA class I, has been shown in a variety of tumors; colon cancer, ovarian cancer, glioblastoma, lymphoma, acute myeloid leukemia (AML), multiple myeloma, melanoma and breast cancer (Table 2). With respect to the presence or absence of HLA, multiple tumor variants exist e.g. tumors with total loss of HLA, loss of only one haplotype, enhanced or reduced expression of specific alleles or loci or combinations thereof $(10,55)$. Especially tumor cells lacking HLA class I while HLA-E expression is maintained or even enhanced will be very difficult to deal with for the immune system; the lack of HLA class I enables them to escape from $T$ cell cytotoxicity while the presence of HLA-E protects them against cytotoxicity by the majority of NK cells expressing NKG2A (Figure 3). The importance of presence or absence of HLA-E when HLA class I is downregulated has been stressed in a study in breast cancer patients where cell surface expression of HLA-E correlated with reduced survival in the group of HLA class I negative patients (56). 


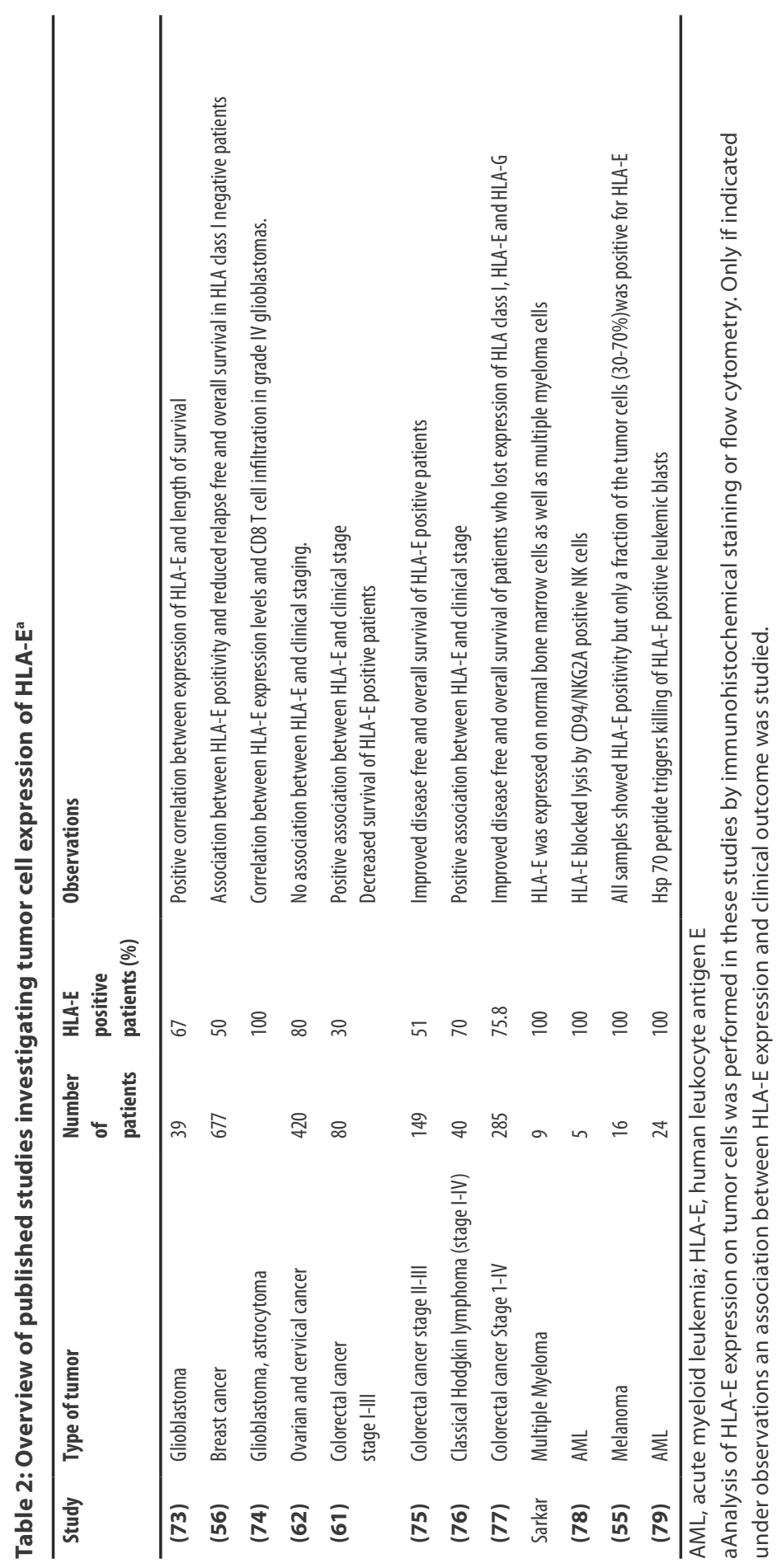




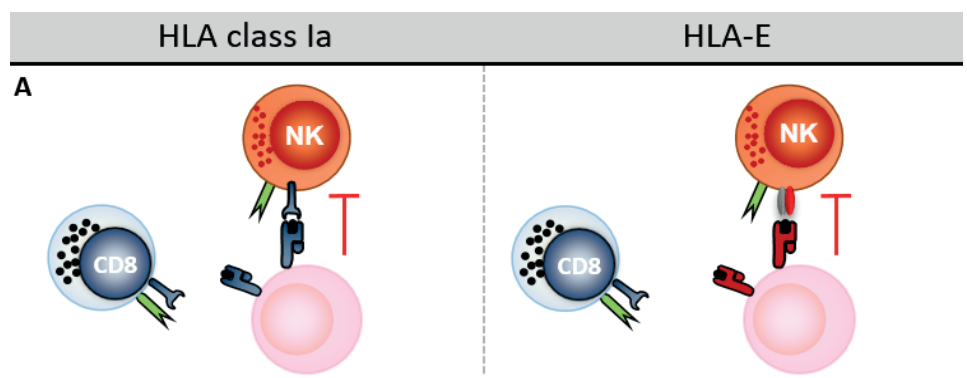

Healthy cell presenting self-peptides $\rightarrow$ no CD8 activation via TCR and inhibition of NK cells via NKG2A or KIR

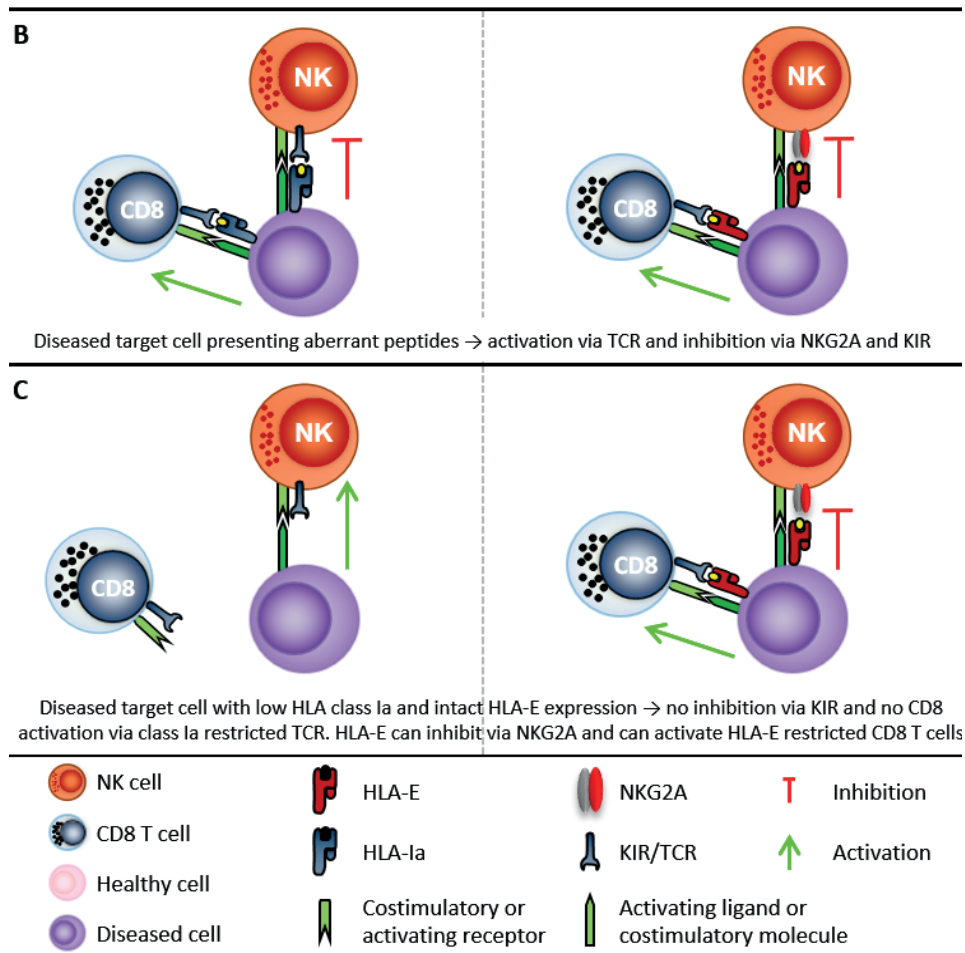

Figure 3. The effect of HLA-E on the cellular immune response in transplantation and cancer. Depicted is the effect of human leukocyte antigen (HLA) on NK cells via CD94/NKG2A or inhibitory killer immunoglobulin-like receptors (KIRs) and CD8 T cells via a $\beta$ Tcell receptor ( $\alpha \beta T C R)$. (A) NK and CD8 T cell tolerance to healthy cells by the presenta- tion of self-peptides via HLA-la and HLA-E. (B) A diseased or allogeneic cell presenting aberrant peptides in intact HLA-la and HLA-E molecules can trigger cytotoxic CD8 T cells while it inhibits the activation of NK cells. (C) Diseased cells lacking HLAla expression do not trigger CD8 T cells but can lead to the activation of NK cells if sufficient levels of disease-associated activating ligands are present. Comparable activation of NK cells can occur upon allogeneic stem cell transplantation (allo-SCT) having a mismatch between KIR and HLA-la. However, when HLA-E expression is maintained (e.g. by virally encoded HLA-E binding peptides) inhibitory signals can be provided via NKG2A present on the majority of peripheral blood NK cells. 
The relevance of HLA-E polymorphism for clinical outcome in cancer has been addressed in only a limited number of studies (Table 3). In a first study comparing 100 patients with nasopharyngeal carcinoma patients and 100 healthy controls, the frequency of individuals having the HLA-E*01:03 allele was higher in the patient group as compared to the healthy controls (72\% vs 57.5\%) (57). However, in a comparable study with 185 nasopharyngeal carcinoma patients and 177 matched controls no significant difference between allele frequencies was found between the groups (58). In line with this study, comparing 100 melanoma patients and 100 healthy controls revealed comparable frequencies of $H L A-E^{*} 01: 01$ and $H L A-E^{*} 01: 03$ alleles between the two groups. Also no significant difference in frequencies of the three genotypes (HLA-E*01:01,01:01, HLA-E*01:01,01:03 and HLA-E*01:03,01:03) was observed (59). In 230 patients with stage II colorectal cancer HLA-E was found to be higher expressed in tumor tissue as compared to normal, surrounding tissue and HLA-E overexpression correlated with a lower disease free survival (60). However, no association between HLA-E genotype and HLA-E expression or disease free- or overall survival was observed. In none of the studies the HLA-E*01:02 and $E^{*} 01: 04$ alleles were detected. These studies indicate that, at this moment, it is not sufficiently clear if and how HLA-E polymorphism effects clinical outcome.

Despite the observation that many tumors show normal or enhanced expression of HLA-E, we and others found that the many tumor cell lines have very low or no HLA-E expression in vitro (Sarkar et al submitted, (54). We recently injected a multiple myeloma cell line expressing very low levels of HLA-E in vitro in immunodeficient mice and observed that HLA-E expression on in vivo grown cells was remarkably higher than expression on in vitro passed cells. Hence, the tumor environment presumably comprises factors that are not present in vitro and that enhance or maintain HLA-E expression. Although the regulatory mechanism for this remain elusive, several tumor-associated factors can be candidates to influence HLA-E expression; for example cellular-stress triggering $\mathrm{Hsp60}$ expression and thus the enhanced availability of $\mathrm{Hs60}$ peptides (6) cytokines (e.g. IFNy) or HLA-G. HLA-G is not expressed on the majority of cells under physiological conditions, but has been shown to be upregulated by a variety of tumors $(56,61)$. Peptides derived from the HLA-G leader can stabilize HLA-E expression on the cell surface and enhance its expression. 


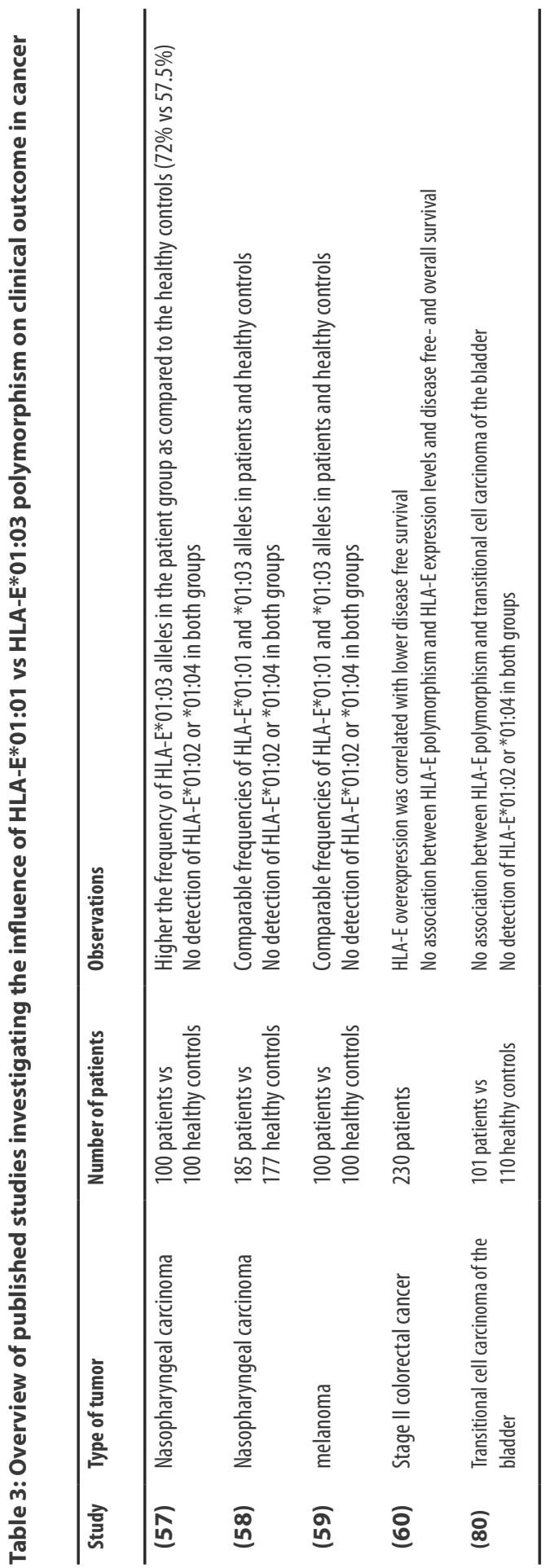


The observation that many tumors maintain expression of HLA-E, even in the absence of classical HLA class I molecules, could suggest a predominantly immunosuppressive role for HLA-E in anti-tumor immunity. Recently, CD8 T cells positive for both CD94/ NKG2A and a 3 TCR have been found to be enriched in biopsies from gynecological- and colorectal cancer and their presence was associated with poor survival $(61,62)$. On the basis of these results it was proposed that HLA-E not only inhibits NK cell anti-tumor responses, but is also detrimental for T cell anti-tumor reactivity via the same CD94/ NKG2A receptor complex. The enhanced presence of CD94/NKG2A positive $T$ cells in the tumor area could imply that these T cells have an increased migratory capacity towards the tumor as compared with T cells not expressing NKG2A. Alternatively, it could indicate that NKG2A expression is de novo induced e.g. by factors associated with tumor itself or its micro-environment; An illustrative example for this phenomenon is the induction of CD94/NKG2A on CD8 T cells by TGF $\beta$ which makes CD8 T cells sensitive for inhibition by HLA-E (63). Evidently, enhanced expression of the inhibitory CD94/NKG2A receptor complex on NK cells and CD8 T cells in combination with the (augmented) expression of HLA-E by tumor cells would be highly detrimental for anti-tumor immune reactivity.

Accumulating evidence suggests that HLA-E can also occur in a soluble form presumably after cleavage from the cell membrane by proteases, and that these soluble HLA-E molecules have immunomodulatory activity. This has also been shown for classical HLA class I, HLA-G and soluble ligands for activating NK cell receptors (e.g. MICA), and has been described to act in an immunosuppressive way by downregulation of activating receptors (e.g. NKG2D for soluble MICA); direct killing of CD8 T or NK effector cells; or by reduction in the levels of cell surface HLA reviewed in (64). Melanoma cells have been shown to shed soluble HLA-E and IFNy enhanced this shedding in vitro (55). Soluble HLA-E has been detected in culture supernatants of 98 cell lines of multiple origins and it was enhanced in serum of melanoma patients as compared with healthy controls (65). Because it was also increased in serum of neuroblastoma patients, it has been proposed to test in larger studies whether soluble HLA-E could serve as diagnostic marker (66).

The presence of HLA-E on the tumor, the observation that tumor associated T cells are mainly NKG2A positive and the fact that the majority of NK cells expresses this receptor, would imply that, in the context of tumors, HLA-E mainly acts in an immunosuppressive manner. If this is indeed the case a better insight in tumorassociated factors controlling HLA-E expression will be indispensable and helpful to develop agents interfering with tumor HLA-E expression. Another strategy could be to block the inhibitory NKG2A receptor using specific antibodies, an approach that is currently being tested for the inhibitory effects of KIR-HLA-C interaction. HLA-E has 
also been shown to reduce effectivity of antibody dependent cellular cytotoxicity (ADCC) resulting from cetuximab binding to colon cancer cells (67). Because NK cells are important mediators of ADCC, interfering with NKG2A-HLA-E interaction will also be crucial for treatment strategies using tumor-specific antibodies aiming at the induction of ADCC.

\section{Parallels between the effect of HLA-E on the cellular immune response in transplantation and in cancer}

The inhibitory or activating effect of HLA-E on NK cell or CD8 T cells in all-SCT or cancer is best characterized for aßTCR on cytotoxic CD8 T cells and KIR or CD94/NKG2A on NK cells. Healthy cells present self-peptides via HLA resulting in immune tolerance (Figure $3 \mathrm{~A}$ ); as a consequence of negative selection in the thymus, presentation of self-peptides will not trigger the activation of CD8 T cells. In addition, the intact HLA molecules on healthy cells will inhibit NK cells via KIR and CD94/NKG2A. Under pathophysiological conditions (e.g. upon viral infection, malignant transformation or an HLA mismatched allograft, a target cell can present aberrant peptides in intact HLA class I and HLA-E molecules (Figure 3B). This presentation of virus or tumorassociated peptides or, upon allo-SCT, peptides from major and minor antigens can trigger cytotoxic CD8 T cells contributing to anti-viral or anti-tumor reactivity but also to unwanted $\mathrm{GvH}$ and HvD responses. On the other hand, the intact HLA molecules will provide an inhibitory signal to NK cells via KIRs (HLA class la) and CD94/NKG2A (HLA-E) which could prevent NK cells from killing and can help virally infected- or tumor cells to escape from NK cell immune surveillance. Importantly, diseased cells frequently express high levels of disease- or stress-associated activating ligands which can overrule inhibitory signaling and activate NK cells. Some diseased target cells may downregulate their HLA class la molecules to escape CD8 T cell recognition (Figure 3C). These low levels of HLA class la reduce the activation threshold of NK cells rendering target cells more susceptible for killing by NK cells. However, when HLA-E expression is maintained (e.g. by virally encoded HLA-E binding peptides) inhibitory signals can be provided via NKG2A expressed by the majority of peripheral blood NK cells. In an HLA mismatched allo-SCT setting, lack of inhibition via KIRs can also occur, namely, in the case of a mismatch between KIRs present on the donor NK cells and HLA class I on the patient cells. However, inhibitory signaling via NKG2A will remain present due to low polymorphic nature of HLA-E. Thus, there is significant overlap between the effects of HLA-E on the cellular immune response in allogeneic stem cell transplantation and in cancer and integrating this knowledge can help to improve the outcome of allo-SCT and cell-based immunotherapy for cancer. 


\section{CONCLUSION}

Evidence is accumulating that HLA-E is more polymorphic and can bind a more extended peptide repertoire than initially thought. Furthermore, novel mechanisms have been identified, e.g. microRNAs, that might provide an additional explanation for aberrant expression of HLA-E during viral infection or malignant transformation. HLA-E interacts with a variety of cells leading to immune activation, upon interaction with activating receptors like the TCR on CD8 T cells or NKG2C on NK cells. Alternatively, immunosuppression which will occur upon binding to inhibitory NKG2A receptors on both T cells and NK cells. Immunosuppression can also occur via the activation of HLA-E restricted regulatory T cells or upon the secretion of soluble HLA-E molecules by virally infected cells, tumor cells or accessory cells like endothelial cells. The exact role of HLA-E and the relevance of HLA-E polymorphism in pathophysiology of viral disease and in GvHD, GvH or anti-tumor responses needs to be further elucidated. Nevertheless, it appears evident that the effects of HLA-E are unique and numerous. Improved understanding of the immunoregulatory function of HLA-E may provide rational for the interference with HLA-E activities and the development of novel therapeutic strategies to improve clinical outcome in transplantation and cancer.

\section{ACKNOWLEDGEMENTS}

The authors would like to thank Dr Mathijs Groeneweg (Maastricht University Medical Center) for generation of Figure 2. This work was supported by a grant from the "kankeronderzoeksfonds Limburg". LW was supported by a personal grant from Dutch Cancer association (KWF kankerbestrijding; UM2012-5375). 


\section{REFERENCES}

1. Sullivan LC, Hoare HL, McCluskey J, Rossjohn J, Brooks AG. A structural perspective on MHC class Ib molecules in adaptive immunity. Trends Immunol 2006: 27: 413-20.

2. van Hall T, Oliveira CC, Joosten SA, Ottenhoff TH. The other Janus face of Qa-1 and HLA-E: diverse peptide repertoires in times of stress. Microbes Infect 2010: 12: 910-8.

3. Sullivan LC, Clements CS, Rossjohn J, Brooks AG. The major histocompatibility complex class Ib molecule HLA-E at the interface between innate and adaptive immunity. Tissue Antigens 2008: 72: 415-24.

4. O'Callaghan $\mathrm{CA}$, Tormo J, Willcox BE, et al. Structural features impose tight peptide binding specificity in the nonclassical MHC molecule HLA-E. Mol Cell 1998: 1: 531-41.

5. Martoglio B, Dobberstein B. Signal sequences: more than just greasy peptides. Trends Cell Biol 1998: 8: 410-5.

6. Michaelsson J, Teixeira de Matos C, Achour A, Lanier LL, Karre K, Soderstrom K. A signal peptide derived from hsp60 binds HLA-E and interferes with CD94/NKG2A recognition. J Exp Med 2002: 196: 1403-14.

7. Iwaszko M, Bogunia-Kubik K. Clinical significance of the HLA-E and CD94/NKG2 interaction. Arch Immunol Ther Exp (Warsz) 2011: 59: 353-67.

8. Strong RK, Holmes MA, Li P, Braun L, Lee N, Geraghty DE. HLA-E allelic variants. Correlating differential expression, peptide affinities, crystal structures, and thermal stabilities. J Biol Chem 2003: 278: 5082-90.

9. Tomasec P, Braud VM, Rickards C, et al. Surface expression of HLA-E, an inhibitor of natural killer cells, enhanced by human cytomegalovirus gpUL40. Science 2000: 287: 1031.

10. Algarra I, Garcia-Lora A, Cabrera T, Ruiz-Cabello F, Garrido F. The selection of tumor variants with altered expression of classical and nonclassical MHC class I molecules: implications for tumor immune escape. Cancer Immunol Immunother 2004: 53: 904-10.

11. Lampen MH, Hassan C, Sluijter M, et al. Alternative peptide repertoire of HLA-E reveals a binding motif that is strikingly similar to HLA-A2. Mol Immunol 2013: 53: 126-31.

12. Nachmani D, Zimmermann A, Oiknine Djian E, et al. MicroRNA editing facilitates immune elimination of HCMV infected cells. PLoS Pathog 2014: 10: e1003963.

13. Castelli EC, Veiga-Castelli LC, Yaghi L, Moreau P. Transcriptional and posttranscriptional regulations of the HLA-G gene. 2014: 2014: 734068.

14. Kulkarni S, Savan R, Qi Y, et al. Differential microRNA regulation of HLA-C expression and its association with HIV control. Nature 2011: 472: 495-8.

15. Braud VM, Allan DS, O'Callaghan CA, et al. HLA-E binds to natural killer cell receptors CD94/ NKG2A, B and C. Nature 1998: 391: 795-9.

16. Wada H, Matsumoto N, Maenaka K, Suzuki K, Yamamoto K. The inhibitory NK cell receptor CD94/ NKG2A and the activating receptor CD94/NKG2C bind the top of HLA-E through mostly shared but partly distinct sets of HLA-E residues. Eur J Immunol 2004: 34: 81-90.

17. Kaiser BK, Barahmand-Pour F, Paulsene W, Medley S, Geraghty DE, Strong RK. Interactions between NKG2x immunoreceptors and HLA-E ligands display overlapping affinities and thermodynamics. J Immunol 2005: 174: 2878-84.

18. Vales-Gomez M, Reyburn HT, Erskine RA, Lopez-Botet M, Strominger JL. Kinetics and peptide dependency of the binding of the inhibitory NK receptor CD94/NKG2-A and the activating receptor CD94/NKG2-C to HLA-E. Embo j 1999: 18: 4250-60.

19. Parham P, Moffett A. Variable NK cell receptors and their MHC class I ligands in immunity, reproduction and human evolution. Nat Rev Immunol 2013: 13: 133-44.

20. Raulet DH, Guerra N. Oncogenic stress sensed by the immune system: role of natural killer cell receptors. Nat Rev Immunol 2009: 9: 568-80. 
21. Llano M, Lee N, Navarro F, et al. HLA-E-bound peptides influence recognition by inhibitory and triggering CD94/NKG2 receptors: preferential response to an HLA-G-derived nonamer. Eur J Immunol 1998: 28: 2854-63.

22. Cheent KS, Jamil KM, Cassidy $\mathrm{S}$, et al. Synergistic inhibition of natural killer cells by the nonsignaling molecule CD94. Proc Natl Acad Sci U S A 2013: 110: 16981-6.

23. Cassidy SA, Cheent KS, Khakoo SI. Effects of Peptide on NK cell-mediated MHC I recognition. Front Immunol 2014: 5: 133.

24. Jabri B, Selby JM, Negulescu H, et al. TCR specificity dictates CD94/NKG2A expression by human CTL. Immunity 2002: 17: 487-99.

25. Moser JM, Gibbs J, Jensen PE, Lukacher AE. CD94-NKG2A receptors regulate antiviral CD8(+) T cell responses. Nat Immunol 2002: 3: 189-95.

26. Guma M, Budt M, Saez A, et al. Expansion of CD94/NKG2C+ NK cells in response to human cytomegalovirus-infected fibroblasts. Blood 2006: 107: 3624-31.

27. Garcia P, Llano M, de Heredia AB, et al. Human T cell receptor-mediated recognition of HLA-E. Eur J Immunol 2002: 32: 936-44.

28. Pietra G, Romagnani C, Manzini C, Moretta L, Mingari MC. The emerging role of HLA-E-restricted $\mathrm{CD} 8+\mathrm{T}$ lymphocytes in the adaptive immune response to pathogens and tumors. J Biomed Biotechnol 2010: 2010: 907092.

29. Pietra G, Romagnani C, Mazzarino P, et al. HLA-E-restricted recognition of cytomegalovirusderived peptides by human CD8+ cytolytic T lymphocytes. Proc Natl Acad Sci U S A 2003: 100: 10896-901.

30. Salerno-Goncalves R, Fernandez-Vina M, Lewinsohn DM, Sztein MB. Identification of a human HLA-E-restricted CD8+ T cell subset in volunteers immunized with Salmonella enterica serovar Typhi strain Ty21a typhoid vaccine. J Immunol 2004: 173: 5852-62.

31. Heinzel AS, Grotzke JE, Lines RA, et al. HLA-E-dependent presentation of Mtb-derived antigen to human CD8+ T cells. J Exp Med 2002: 196: 1473-81.

32. Hu D, Ikizawa K, Lu L, Sanchirico ME, Shinohara ML, Cantor H. Analysis of regulatory CD8 T cells in Qa-1-deficient mice. Nat Immunol 2004: 5: 516-23.

33. Jiang H, Canfield SM, Gallagher MP, et al. HLA-E-restricted regulatory CD8(+) T cells are involved in development and control of human autoimmune type 1 diabetes. J Clin Invest 2010: 120: 3641-50.

34. Liu XX, Pan FH, Tian W. Characterization of HLA-E polymorphism in four distinct populations in Mainland China. Tissue Antigens 2012: 80: 26-35.

35. Grimsley C, Kawasaki A, Gassner C, et al. Definitive high resolution typing of HLA-E allelic polymorphisms: Identifying potential errors in existing allele data. Tissue Antigens 2002: 60: 206-12.

36. Arnaiz-Villena A, Vargas-Alarcon G, Serrano-Vela Jl, et al. HLA-E polymorphism in Amerindians from Mexico (Mazatecans), Colombia (Wayu) and Chile (Mapuches): evolution of MHC-E gene. Tissue Antigens 2007: 69 Suppl 1: 132-5.

37. Grimsley C, Ober C. Population genetic studies of HLA-E: evidence for selection. Hum Immunol 1997: 52: 33-40.

38. Matte C, Lacaille J, Zijenah L, Ward B, Roger M. HLA-G and HLA-E polymorphisms in an indigenous African population. The ZVITAMBO Study Group. Hum Immunol 2000: 61: 1150-6.

39. Felicio LP, Porto IO, Mendes-Junior CT, et al. Worldwide HLA-E nucleotide and haplotype variability reveals a conserved gene for coding and $3^{\prime}$ untranslated regions. Tissue Antigens 2014: 83: 82-93.

40. Pacasova R, Martinozzi S, Boulouis HJ, et al. Cell-surface expression and alloantigenic function of a human nonclassical class I molecule (HLA-E) in transgenic mice. J Immunol 1999: 162: 5190-6. 
41. Romagnani C, Pietra G, Falco M, et al. Identification of HLA-E-specific alloreactive T lymphocytes: a cell subset that undergoes preferential expansion in mixed lymphocyte culture and displays a broad cytolytic activity against allogeneic cells. Proc Natl Acad Sci U S A 2002: 99: 11328-33.

42. Tamouza R, Rocha V, Busson M, et al. Association of HLA-E polymorphism with severe bacterial infection and early transplant-related mortality in matched unrelated bone marrow transplantation. Transplantation 2005: 80: 140-4.

43. Tamouza R, Busson M, Rocha V, et al. Homozygous status for HLA-E*0103 confers protection from acute graft-versus-host disease and transplant-related mortality in HLA-matched sibling hematopoietic stem cell transplantation. Transplantation 2006: 82: 1436-40.

44. Danzer M, Polin H, Proll J, et al. Clinical significance of HLA-E*0103 homozygosity on survival after allogeneic hematopoietic stem-cell transplantation. Transplantation 2009: 88: 528-32.

45. Ludajic K, Rosenmayr A, Fae I, et al. Association of HLA-E polymorphism with the outcome of hematopoietic stem-cell transplantation with unrelated donors. Transplantation 2009: 88: 12278.

46. Hosseini E, Schwarer AP, Ghasemzadeh M. The impact of HLA-E polymorphisms in graft-versushost disease following HLA-E matched allogeneic hematopoietic stem cell transplantation. Iran J Allergy Asthma Immunol 2012: 11: 15-21.

47. Hosseini E, Schwarer AP, Jalali A, Ghasemzadeh M. The impact of HLA-E polymorphisms on relapse following allogeneic hematopoietic stem cell transplantation. Leuk Res 2013: 37: 516-9.

48. Furst D, Bindja J, Arnold R, et al. HLA-E polymorphisms in hematopoietic stem cell transplantation. Tissue Antigens 2012: 79: 287-90.

49. Foley B, Cooley S, Verneris MR, et al. Cytomegalovirus reactivation after allogeneic transplantation promotes a lasting increase in educated NKG2C+ natural killer cells with potent function. Blood 2012: 119: 2665-74.

50. Al-Lamki RS, Bradley JR, Pober JS. Endothelial cells in allograft rejection. Transplantation 2008: 86: $1340-8$.

51. Coupel S, Moreau A, Hamidou M, Horejsi V, Soulillou JP, Charreau B. Expression and release of soluble HLA-E is an immunoregulatory feature of endothelial cell activation. Blood 2007: 109: 2806-14.

52. Schreiber RD, Old LJ, Smyth MJ. Cancer immunoediting: integrating immunity's roles in cancer suppression and promotion. Science 2011:331: 1565-70.

53. del Campo AB, Carretero J, Aptsiauri N, Garrido F. Targeting HLA class I expression to increase tumor immunogenicity. Tissue Antigens 2012: 79: 147-54.

54. Palmisano GL, Contardi E, Morabito A, Gargaglione V, Ferrara GB, Pistillo MP. HLA-E surface expression is independent of the availability of HLA class I signal sequence-derived peptides in human tumor cell lines. Hum Immunol 2005: 66: 1-12.

55. Derre L, Corvaisier M, Charreau B, et al. Expression and release of HLA-E by melanoma cells and melanocytes: potential impact on the response of cytotoxic effector cells. J Immunol 2006: 177: 3100-7.

56. de Kruijf EM, Sajet A, van Nes JG, et al. HLA-E and HLA-G expression in classical HLA class I-negative tumors is of prognostic value for clinical outcome of early breast cancer patients. J Immunol 2010: 185: 7452-9.

57. Hirankarn N, Kimkong I, Mutirangura A. HLA-E polymorphism in patients with nasopharyngeal carcinoma. Tissue Antigens 2004: 64: 588-92.

58. Hassen $E$, Ghedira R, Ghandri N, et al. Lack of association between human leukocyte antigen-E alleles and nasopharyngeal carcinoma in Tunisians. DNA Cell Biol 2011: 30: 603-9.

59. Moya-Quiles MR, Martinez-Escribano J, Guerra-Perez N, et al. Lack of association between HLA-E polymorphism and primary cutaneous melanoma in Spanish patients. J Dermatol Sci 2005: 40: $62-4$. 
60. Zhen ZJ, Ling JY, Cai Y, Luo WB, He YJ. Impact of HLA-E gene polymorphism on HLA-E expression in tumor cells and prognosis in patients with stage III colorectal cancer. Med Oncol 2013: 30: 482.

61. Bossard C, Bezieau S, Matysiak-Budnik T, et al. HLA-E/beta2 microglobulin overexpression in colorectal cancer is associated with recruitment of inhibitory immune cells and tumor progression. Int J Cancer 2012: 131: 855-63.

62. Gooden M, Lampen M, Jordanova ES, et al. HLA-E expression by gynecological cancers restrains tumor-infiltrating CD8(+) T lymphocytes. Proc Natl Acad Sci U S A 2011: 108: 10656-61.

63. Bertone S, Schiavetti F, Bellomo R, et al. Transforming growth factor-beta-induced expression of CD94/NKG2A inhibitory receptors in human T lymphocytes. Eur J Immunol 1999: 29: 23-9.

64. Campoli M, Ferrone S. Tumor escape mechanisms: potential role of soluble HLA antigens and NK cells activating ligands. Tissue Antigens 2008: 72: 321-34.

65. Allard M, Oger R, Vignard V, et al. Serum soluble HLA-E in melanoma: a new potential immunerelated marker in cancer. PLoS One 2011: 6: e21118.

66. Morandi F, Cangemi G, Barco S, et al. Plasma levels of soluble HLA-E and HLA-F at diagnosis may predict overall survival of neuroblastoma patients. Biomed Res Int 2013: 2013: 956878.

67. Levy EM, Sycz G, Arriaga JM, et al. Cetuximab-mediated cellular cytotoxicity is inhibited by HLA-E membrane expression in colon cancer cells. Innate Immun 2009: 15: 91-100.

68. Berman HM, Westbrook J, Feng Z, et al. The Protein Data Bank. Nucleic Acids Res 2000: 28: 235 42.

69. Arnold K, Bordoli L, Kopp J, Schwede T. The SWISS-MODEL workspace: a web-based environment for protein structure homology modelling. Bioinformatics 2006: 22: 195-201.

70. Biasini M, Bienert S, Waterhouse A, et al. SWISS-MODEL: modelling protein tertiary and quaternary structure using evolutionary information. Nucleic Acids Res 2014: 42: W252-8.

71. Bordoli L, Kiefer F, Arnold K, Benkert P, Battey J, Schwede T. Protein structure homology modeling using SWISS-MODEL workspace. Nat Protoc 2009: 4: 1-13.

72. Guex N, Peitsch MC, Schwede T. Automated comparative protein structure modeling with SWISS-MODEL and Swiss-PdbViewer: a historical perspective. Electrophoresis 2009: 30 Suppl 1: S162-73.

73. Kren L, Slaby O, Muckova K, et al. Expression of immune-modulatory molecules HLA-G and HLA-E by tumor cells in glioblastomas: an unexpected prognostic significance? Neuropathology 2011: 31: 129-34.

74. Mittelbronn M, Simon P, Loffler C, et al. Elevated HLA-E levels in human glioblastomas but not in grade I to III astrocytomas correlate with infiltrating CD8+ cells. J Neuroimmunol 2007: 189: 50-8.

75. Benevolo $M$, Mottolese $M$, Tremante $E$, et al. High expression of HLA-E in colorectal carcinoma is associated with a favorable prognosis. J Transl Med 2011: 9: 184.

76. Kren L, Fabian P, Slaby O, et al. Multifunctional immune-modulatory protein HLA-E identified in classical Hodgkin lymphoma: possible implications. Pathol Res Pract 2012: 208: 45-9.

77. Zeestraten EC, Reimers MS, Saadatmand S, et al. Combined analysis of HLA class I, HLA-E and HLA-G predicts prognosis in colon cancer patients. Br J Cancer 2014: 110: 459-68.

78. Nguyen S, Dhedin N, Vernant JP, et al. NK-cell reconstitution after haploidentical hematopoietic stem-cell transplantations: immaturity of NK cells and inhibitory effect of NKG2A override GvL effect. Blood 2005: 105: 4135-42.

79. Gross C, Holler E, Stangl S, et al. An Hsp70 peptide initiates NK cell killing of leukemic blasts after stem cell transplantation. Leuk Res 2008: 32: 527-34.

80. Veiga-Castelli LC, de Paula Cruz AS, Inacio MM, et al. Lack of association between HLA-E polymorphisms and transitional cell carcinoma of the bladder. Tissue Antigens 2013: 82: 197200. 
116 | Chapter 5 
NKG2A expression is not per se detrimental for the anti-multiple myeloma activity of activated natural killer cells in an in vitro system mimicking the

\section{NKG2A expression is not per se detrimental for the anti-multiple myeloma activity of activated natural killer cells in an in vitro system mimicking the tumor microenvironment}

Niken M. Mahaweni1,2, Femke A. I. Ehlers1,2, Subhashis Sarkar1, Johanna W. H. Janssen3, Marcel G. J. Tilanus2, Gerard M. J. Bos1, Lotte Wieten2*

1 Department of Internal Medicine, Division of Hematology, Maastricht University Medical Center+, Maastricht, The Netherlands; GROW School for Oncology and Developmental Biology, Maastricht University, Maastricht, The Netherlands 2 Department of Transplantation Immunology, Tissue Typing Laboratory, Maastricht University Medical Center+, Maastricht, The Netherlands; GROW School for Oncology and Developmental Biology, Maastricht University, Maastricht, The Netherlands 3 Department of Clinical Genetics, Maastricht University Medical Center+, Maastricht, The Netherlands

Front. Immunol. 2018 June. doi: 10.3389/fimmu.2018.01415 


\section{ABSTRACT}

Natural Killer (NK) cell-based immunotherapy is a promising therapy for cancer patients. Inhibitory killer immunoglobulin-like receptors (KIRs) and NKG2A are required for NK cell licensing but can also inhibit NK cell effector function. Upon reconstitution in a stem cell transplantation setting or after ex vivo NK expansion with IL-2, NKG2A is expressed on a large percentage of NK cells. Since the functional consequences of NKG2A co-expression for activated NK cells are not well known, we compared NKG2A+ vs NKG2A- NK cell subsets in response to K562 cells, multiple myeloma (MM) cell lines and primary MM cells. NK cells were isolated from healthy donors (HLA-C1+C2+Bw4+) and activated overnight with 1000U/ml IL-2. NK cell degranulation in subsets expressing KIRs and/or NKG2A was assessed at 21 or $0.6 \%$ $\mathrm{O}_{2}$. Activated NKG2A+ NK cell subsets degranulated more vigorously than NKG2Asubsets both at 21 and $0.6 \%$ O2. This was irrespective of the presence of KIR and occurred in response to HLA deficient K562 cells as well as HLA competent, lowly expressing HLA-E MM cell lines. In response to primary MM cells, no inhibitory effects of NKG2A were observed, and NKG2A blockade did not enhance degranulation of NKG2A+ subsets. KIR- NK cells expressing NKG2A degranulated less than their NKG2A- counterparts in response to MM cells having high levels of peptideinduced membrane HLA-E, suggesting that high surface HLA-E levels are required for NKG2A to inhibit activated NK cells. Addition of daratumumab, an anti-CD38 to trigger antibody-dependent cellular cytotoxicity (ADCC), improved the anti-MM response for all subsets and degranulation of the KIR-NKG2A- "unlicensed" subset was comparable to KIR+ or NKG2A+ licensed subsets. This demonstrates that with potent activation, all subsets can contribute to tumor clearance. Additionally, subsets expressing KIRs mismatched with the HLA ligands on the target cell had the highest level of activation in response to MM cell lines as well as against primary MM. Our current study demonstrated that if NK cells are sufficiently activated, e.g. via cytokine or antibody activation, the (co-) expression of NKG2A receptor may not necessarily be a disadvantage for NK cell-based therapy. 
NKG2A expression is not per se detrimental for the anti-multiple myeloma

activity of activated natural killer cells in an in vitro system mimicking the

tumor microenvironment

\section{INTRODUCTION}

Natural killer (NK) cell-based immunotherapy is an attractive novel therapy against cancer owing to its target selectivity and killing potential [1]. NK cells are armed with both activating and inhibitory receptors, and their activation is dependent on the balance between activating and inhibitory signals. The major inhibitory receptors, killer immunoglobulin-like receptors (KIRs) and the NKG2A receptor, provide NK cells with inhibitory signals and are involved in the education process of an NK cell $[2,3]$. This NK cell education process, also known as licensing, plays a pivotal role in shaping the NK cell ability to kill a target cell. Previous studies on murine NK cells have demonstrated that the number of inhibitory receptors for self-major histocompatibility complex (MHC) expressed on NK cells is proportionate to the strength of NK cell responsiveness against a target cell [4, 5]. A more recent study has shown that this is also relevant for human NK cells [6]. Moreover, they observed that NKG2A has a stronger licensing impact compared to the KIRs without a significant difference between KIR2DL2/3, KIR2DL1, and KIR3DL1.

In the context of the NK cell response against tumor cells, inhibitory receptors have a dual role: On the one hand, having more inhibitory receptors, and thus better licensed and potentially more potent NK cells, could be advantageous for the NK cell response against $\mathrm{MHC/HLA}$-class I deficient tumor cells. On the other hand, a licensed NK cell could be inhibited when binding to its cognate ligand expressed on an $\mathrm{MHC}$ / HLA- class I competent tumor cell unless an excessive amount of activating signals is present [7]. To reduce the inhibitory effects mediated by KIRs, donor-derived, alloreactive, KIR-ligand mismatched NK cells have been proposed as one of the solutions to achieve a better response against tumor cells. Such donor NK cells would namely be fully licensed, albeit, their anti-tumor reactivity would not be hampered due to the genetic incompatibility between donor KIR and patient HLA ligands [8, 9].

In contrast to the KIRs, mismatching for NKG2A and its HLA-E ligand is not possible due to the limited polymorphism of HLA-E. NKG2A can, however, be an important inhibitory receptor for NK cells as it has been shown that NKG2A can inhibit antitumor reactivity of NKG2A+ NK cells and NKG2A blocking antibodies could improve the anti-tumor response [10]. Moreover, NKG2A is expressed on a large fraction of the NK cells $(20-80 \%)[11,12]$, and this percentage is even higher on reconstituting relatively immature NK cells upon allogeneic stem cell transplantation [13]. Also, NKG2A has been shown to be overexpressed on NK cells isolated from chronic lymphocytic leukemia patients [14]. 
Our group aims to develop NK cell-based immunotherapy for multiple myeloma, a hematological malignancy characterized by the growth of malignant plasma cells in the bone marrow for which curative treatment options are currently lacking. We previously reported that both primary MM and MM cell lines express HLA-ABC and HLA-E [15]. Additionally, we demonstrated that NKG2A negative KIR-ligand mismatched NK cells were more effective against HLA-class I competent multiple myeloma (MM) cell lines compared to NKG2A negative KIR-ligand matched NK cells [15], also under a more suppressive tumor microenvironment [16]. Also, we showed that an ADCC-triggering antibody, like daratumumab, can enhance the NK anti-MM response and that having a KIR-ligand mismatch can further potentiate the response [16].

Although several of the above-mentioned studies illustrate the functional relevance of either KIRs or NKG2A to set the NK cell activation threshold, the effect of co-expression of these receptors on the single NK cell level remains largely unexplored. As NKG2A is (co-)expressed on many NK cells, including KIR positive subsets, we follow up on our previous findings by investigating whether (co-)expression of NKG2A is beneficial, due to enhanced NK cell licensing, or detrimental due to inhibitory interactions with HLA-E- for the NK cell anti-MM response. We compare NK subsets with vs without NKG2A in three different settings: 1 ) in response to HLA deficient target cells, 2) in response to HLA competent target cells and 3 ) in the presence of ADCC-triggering antibodies. As we intend to perform future clinical studies with activated NK cells and the effect of NKG2A co-expression on activated NK cells remains largely elusive, we activated the NK cells throughout the study with IL-2. Additionally, to explore the influence of tumor microenvironment on the process of NK cell activation, we performed the experiments in the presence of ambient air $(21 \%)$ or low $(0.6 \%)$ oxygen concentration. This oxygen concentration is selected from previous experiments [17] and relevant for tumor hypoxia setting. 
NKG2A expression is not per se detrimental for the anti-multiple myeloma activity of activated natural killer cells in an in vitro system mimicking the

tumor microenvironment

\section{MATERIALS AND METHODS}

\section{Cell lines and culture}

K562 cell line was cultured in IMDM and 10\% fetal calf serum (FCS). UM-9, RPMI8226/ s-luc, U266, and, RPMI8226/s cell line were cultured in RPMI1640 and 10\% FCS. JJN-3 cell line was cultured in 40\% IMDM, 40\% low glucose DMEM and 20\% FCS. All cell culture media were supplemented with $100 \mathrm{U} / \mathrm{mL}$ penicillin (Gibco) and $100 \mu \mathrm{g} / \mathrm{mL}$ streptomycin (Gibco). K562 and U266 cell line were purchased from American Type Culture Collection (ATCC, USA). UM-9 and RPMI8226/s-luc cell line were gifts from Dr. A. Martens, Vrije Universiteit Medisch Centrum, The Netherlands. RPMI8226/s and $J J N-3$ cell line were purchased from Deutsche Sammlung von Mikroorganismen und Zellkulturen (DSMZ, Germany). All media were from Gibco, Breda, The Netherlands and FCS was produced by Greiner Bio-One International, Gmbh. Cell lines were cultured at $37^{\circ} \mathrm{C}$ in humidified air containing $5 \% \mathrm{CO}_{2}$ with $21 \% \mathrm{O}_{2}$ (Sanyo MCO-20AIC, Sanyo Electric Co, Japan).

\section{Primary multiple myeloma cells}

Primary multiple myeloma cells were obtained from the department of cytogenetics as leftover material from a patient subject to a cytogenetic examination. Under the Dutch law on Research Involving Human Subject (http://www.ccmo.nl/en/non-wmoresearch), leftover materials from a patient can be used for research and are waived from individual patient's consent. MM cells were purified using CD138 beads positive selection according to manufacturer instruction (Miltenyi Biotech). The purified cells were resuspended in RPMI 1640 and 10\% FCS supplemented with $100 \mathrm{U} / \mathrm{mL}$ penicillin and immediately used in degranulation assay

\section{HLA genotyping, NK cell donor selection, and analysis of KIR-ligand matched/mismatched status}

The genotypic expression of HLA epitopes relevant for KIR2DL1 (HLA group C2); KIR2DL2/3 (HLA group C1) or KIR3DL1 (HLA-Bw4 and HLA-A*23, $-A^{*} 24,-A^{*} 32$ ) in cell lines and healthy blood donors was determined using Luminex ${ }^{\circledR}$ sequence-specific oligonucleotides (SSO) analysis (One Lambda). Based on the genotyping result: UM9, U266, and JJN-3 were HLA-C1+C2-Bw4- and RPMI8226/s was HLA-C1+C2+Bw4-. KIR-ligand matched NK cells for UM9, U266, and JJN-3 were, therefore, KIR2DL2/3 positive. KIR-ligand mismatched NK cells for UM9, U266, and JJN-3 were KIR2DL1 positive and/or KIR3DL1 positive. For RPMI8226/s, KIR-ligand matched NK cells were KIR2DL2/3 and/or KIR2DL1 positive. KIR-ligand mismatched NK cells for RPMI8226/s were KIR3DL1 positive. NK cell donors were healthy volunteers or buffy coats with genotype HLA-C1+C2+Bw4+ and phenotypically expressing KIR2DL1, KIR2DL2/3, 
and KIR3DL1. All donors signed informed consent forms. The use of buffy coats, being a by-product of a required Medical Ethical Review Committee (METC) procedure, does not need ethical approval in the Netherlands under the Dutch Code for Proper Secondary Use of Human Tissue.

\section{NK cell isolation}

NK cells were isolated by negative selection of NK cells isolation kit using MACS beads and columns according to manufacturer's protocol (Miltenyi Biotec, $\mathrm{GmbH}$ ). For all experiments, NK cells were activated overnight with $1000 \mathrm{IU} / \mathrm{ml}$ recombinant human IL-2 (Proleukin, Novartis) in RPMI-1640 medium (Gibco) supplemented with $10 \%$ fetal calf serum (Greiner Bio-One), $100 \mathrm{U} / \mathrm{mL}$ penicillin (Gibco) and $100 \mu \mathrm{g} / \mathrm{mL}$ streptomycin (Gibco) at $37^{\circ} \mathrm{C}$ in humidified air containing $5 \% \mathrm{CO}_{2}$ with $21 \% \mathrm{O}_{2}$ (Sanyo MCO-20AIC, Sanyo Electric Co, Japan).

\section{CD107a degranulation assay}

To assess NK cell degranulation, CD107a expression on NK cells was analyzed using flow cytometry-based assay. For this, target cells (tumor cells) were plated in 24 wells plates at a concentration of $2 \times 10^{6}$ cells $/ \mathrm{mL}$ per well and incubated overnight at $37^{\circ} \mathrm{C}$ in humidified air containing $5 \% \mathrm{CO}_{2}$ with $21 \% \mathrm{O}_{2}$ (Sanyo MCO-20AIC, Sanyo Electric Co, Japan) or $0.6 \% \mathrm{O}_{2}$ (except experiment in Figure 5) (Invivo, 1000 Ruskinn Technology Ltd, Bridgend, UK). Prior to the assay, IL-2 activated NK cells were harvested and washed and when indicated in the experiment, subjected to pre-incubation with $50 \mathrm{mM}$ sodium L-lactate (Sigma) or $100 \mathrm{ng} / \mathrm{mL}$ prostaglandin E2 (Sigma) or medium (Figure 1 and Figure S1 in Supplementary Material), otherwise NK cells were immediately co-cultured with tumor cells in the assay without pre-incubation. For the NKG2A blocking assay, NK cells were incubated with $1 \mu \mathrm{g} / \mathrm{mL}$ anti-NKG2A antibody (clone: Z199, Beckman Coulter) (Figure 3C) or anti-NKG2A-PE-Cy7 (clone: REA110, Miltenyi Biotec) (Figure 5D) for 1 hour in $37^{\circ} \mathrm{C}$ in humidified air containing $5 \% \mathrm{CO}_{2}$ with $21 \%$ $\mathrm{O}_{2}$ or $0.6 \mathrm{O}_{2}$ when indicated in the figure. For the HLA-E blocking assay, target cells were incubated with $10 \mu \mathrm{g} / \mathrm{mL}$ anti-HLA-E antibody (clone: 3D12HLA-E, eBioscience) (Figure $5 \mathrm{C}$ and Figure $\mathrm{S} 6$ in Supplementary Material) 30 minutes in $37^{\circ} \mathrm{C}$ in humidified air containing $5 \% \mathrm{CO}_{2}$ with $21 \% \mathrm{O}_{2}$ or $0.6 \mathrm{O}_{2}$ when indicated in the figure. For the ADCC assay (Figure 4), tumor cells were pre-incubated for 30 minutes with $1 \mu \mathrm{g} / \mathrm{mL}$ daratumumab or medium at $21 \% \mathrm{O}_{2}$ or $0.6 \% \mathrm{O}_{2}$ before co-cultured with NK cells. NK cells exposed to tumor microenvironmental factors (TMEF) were then, in duplicate wells, co-cultured with the target cells and $2 \mu \mathrm{l}$ anti-CD107a-Horizon V450 (clone: $\mathrm{H} 4 \mathrm{~A} 3, \mathrm{BD}$ ) was added per well. After 1 hour of coculture, monensin (BD) was added. After another 3 hours, the plate was placed on ice to stop the reaction. Cells were then stained on ice with anti-CD3-APC/H7 (SK7, BD), anti-CD56-PeCy7 (clone: B159, BD), 
NKG2A expression is not per se detrimental for the anti-multiple myeloma activity of activated natural killer cells in an in vitro system mimicking the

\section{tumor microenvironment}

anti-KIR2DL1-APC (clone: 143211, R\&D), anti-KIR2DL2/3/S2-PE (clone: DX27, Miltenyi Biotec), anti-KIR3DL1-FITC (clone: DX9, Miltenyi Biotec) and anti-NKG2A-PC5.5 (clone: Z199, Beckman Coulter). To analyze different subsets of NK cell, CD3- CD56+ cells were gated followed by gating of NKG2A- and NKG2A+ population and further gating based on the KIRs expressions.

A

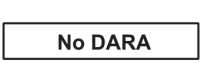

B

C

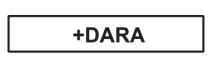

D

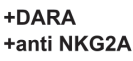

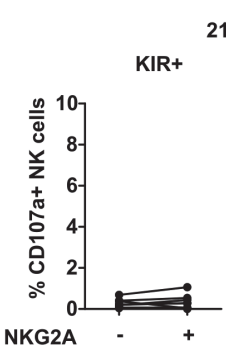

NKG2A

$21 \% \mathrm{O}_{2}$

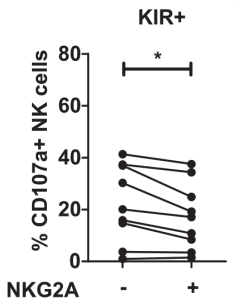

KIR+ $21 \% \mathrm{O}_{2}$ KIR-

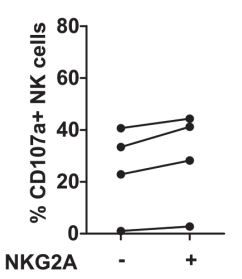

KIR+

$21 \%$

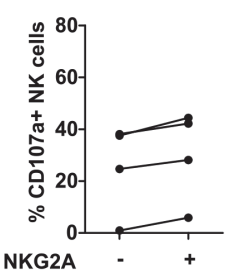

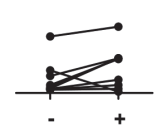

KIR-
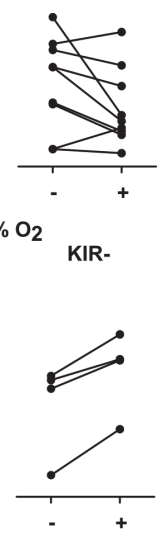

KIR-

KIR-

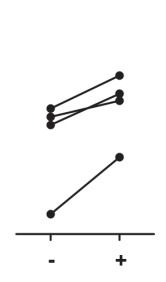

$0.6 \% \mathrm{O}_{2}$

KIR+ KIR-

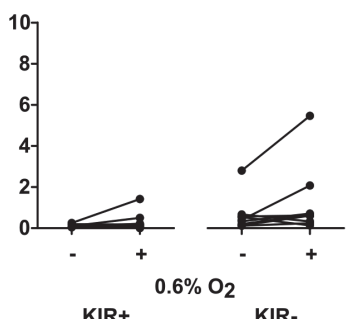

KIR+

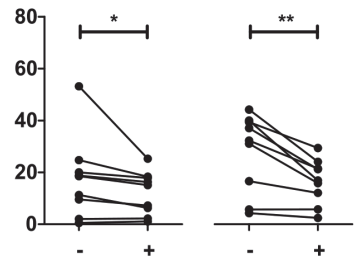

$\mathrm{KIR+} \quad 0.6 \% \mathrm{O}_{2}$ KIR-
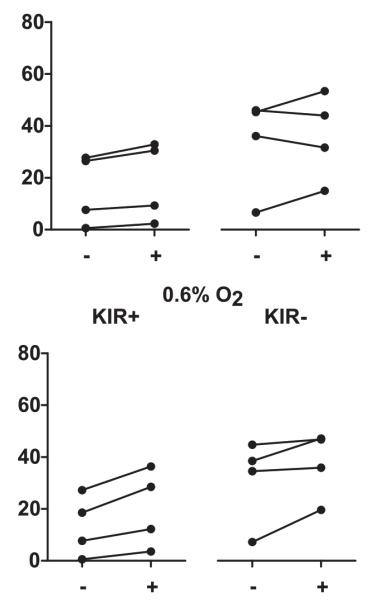

Figure 5. Effect of NKG2A and KIR expression on daratumumab-induced NK cell fratricide. NK cells were incubated at $21 \%$ or $0.6 \% \mathrm{O}_{2}$ for 5 hours in the absence (A) or presence of daratumumab (DARA) (B), or DARA and anti HLA-E (C) or DARA and anti NKG2A (D). Flow cytometry was used to subtype NK cells based on their expression of NKG2A and KIRs. Degranulating NK cells were denoted as CD107a+ NK cells. Each dot represents the average of a technical replicate. $\left({ }^{*}=p<0.05,{ }^{* *}=p<0.01\right)$. $\mathrm{n}=9$ different donors (A\&B) or 4 donors (C\&D). 


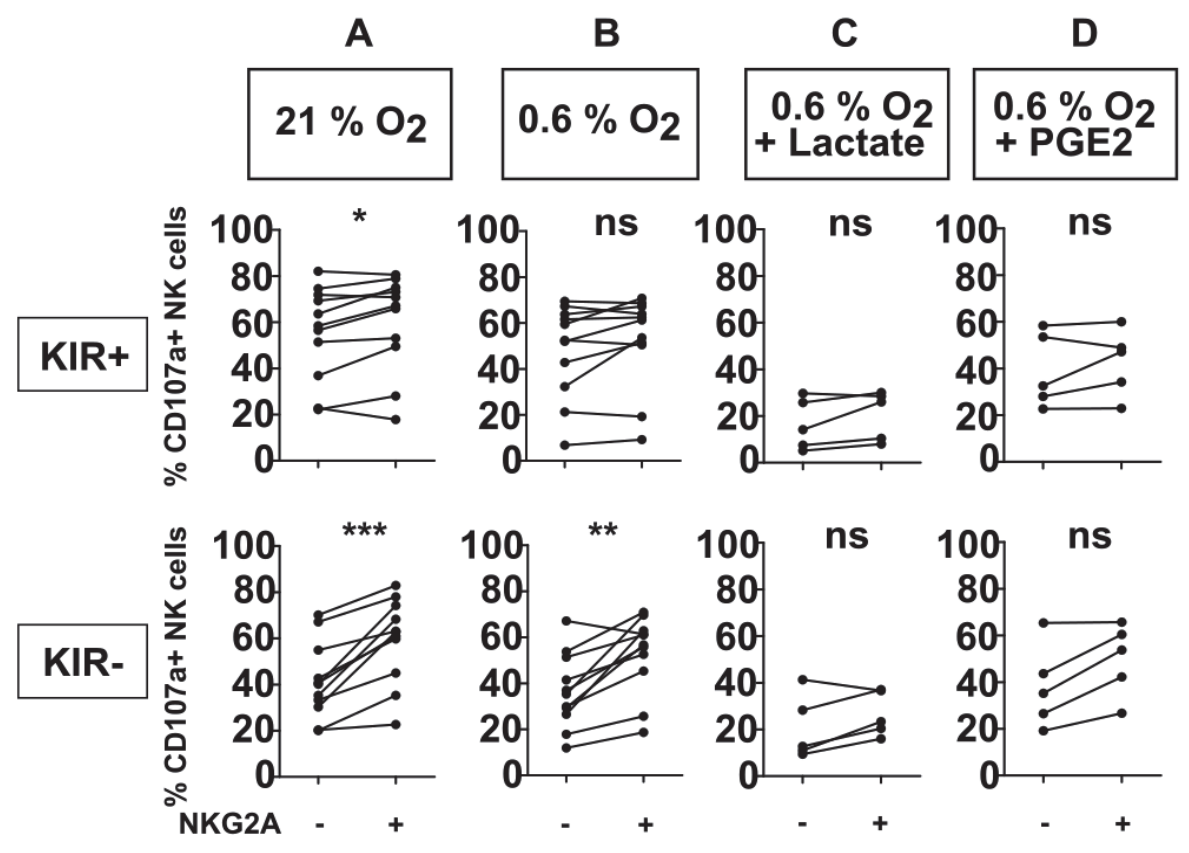

Figure 1. Effect of NKG2A on NK cell degranulation in the presence of different microenvironment factors. NK cells were co-cultured with target cells (K562 cells) in a 1:1 E:T ratio for 4 hours at 21 (A) or $0.6 \% \mathrm{O}_{2}(\mathrm{~B})$, or combinations of $0.6 \% \mathrm{O}_{2}$ and $50 \mathrm{mM}$ lactate $(\mathrm{C})$, or $100 \mathrm{ng} / \mathrm{mL}$ PGE2 (D). Flow cytometry was used to subtype NK cells based on their expression of NKG2A and KIRs. The percentage of degranulating NK cells is shown as \% CD107a+ NK cells. Each dot represents an average of a technical replicate from an individual NK cell donor. (A) and (B) $n=11$ donors, (C) and (D) $n=5$ donors tested in independent experiments ( $n s=$ not significant, ${ }^{*}=p<0.05,{ }^{* *}=p<0.01,{ }^{* * *}=p<0.001$ ) 
NKG2A expression is not per se detrimental for the anti-multiple myeloma activity of activated natural killer cells in an in vitro system mimicking the

tumor microenvironment

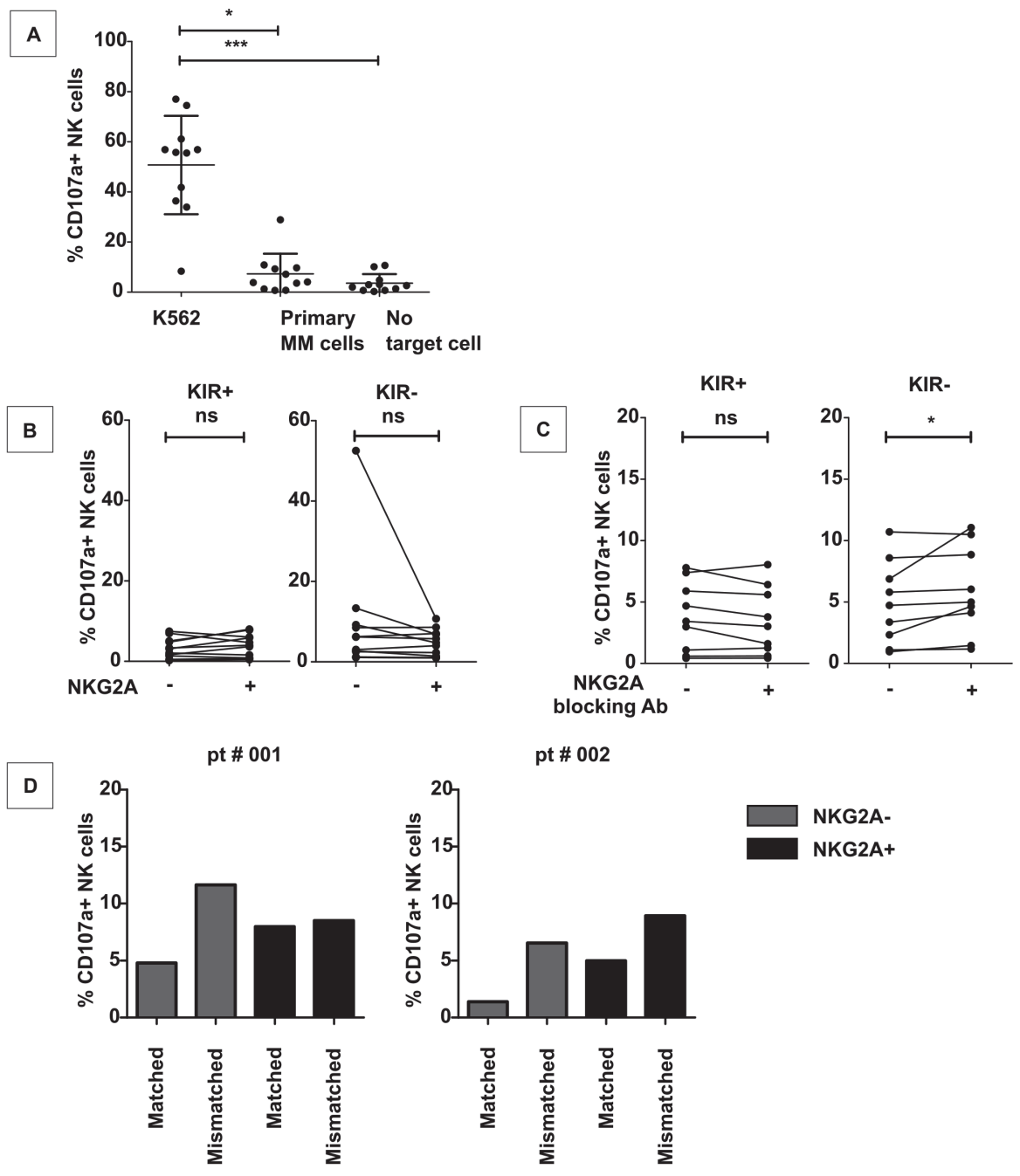

Figure 3. Effect of NKG2A expression on NK cells on NK cell activation against MM cell lines and primary MM cells. IL-2 activated NK cells were co-cultured with K562 cell line (A), primary MM (A, B, C, D) or without target cells (A) for 4 hours in a degranulation assay with or without an NKG2A blocking antibody (C). Flow cytometric analysis was used to subtype NK cells based on their expression of NKG2A and KIRs. Degranulating NK cells were denoted as CD107a+ NK cells. Each dot represents an average of a technical replicate. $(A-C) n=10$ independent experiments with samples from 10 different MM patients as target cells $(D) n=2$ different myeloma patients used as target cells (ns= not significant, ${ }^{*}=p<0.05,{ }^{* *}=p<0.01,{ }^{* * *}=p<0.001$ ) 


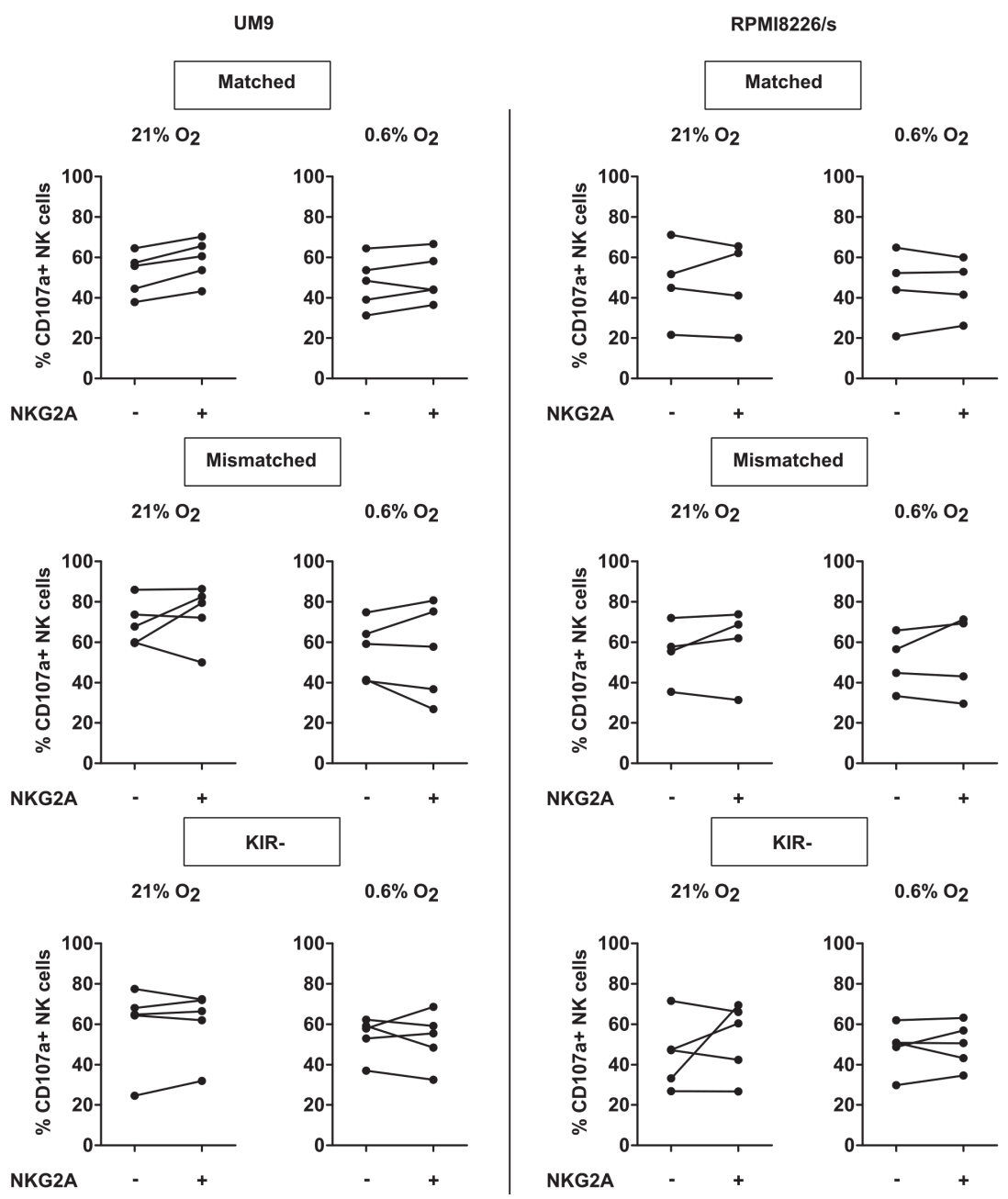

Figure 4. Effect of NKG2A co-expression on daratumumab-induced ADCC. UM9 or RPMI8226/s cells were pre-incubated with daratumumab for 30 minutes before adding IL-2 activated NK cells at a 1:1 E:T ratio. A degranulation assay was performed for 4 hours at $21 \%$ or $0.6 \% \mathrm{O}_{2}$. Flow cytometric analysis was used to subtype NK cells based on their expression of NKG2A and KIRs. Degranulating NK cells were denoted as CD107a+ NK cells. Each dot represents an average of a technical per donor. $\mathrm{n}=$ 5 independent experiments with 5 different donors and two different cell lines. 
NKG2A expression is not per se detrimental for the anti-multiple myeloma activity of activated natural killer cells in an in vitro system mimicking the

tumor microenvironment

\section{Induction of HLA-E expression using HLA leader peptides}

U266 cells were incubated with $500 \mu \mathrm{M}$ of HLA-A1 (VMAPRTLLL), HLA-B7 (VMAPRTVLL) or a non HLA-E binding control peptide (RGPGRAFVTI) (Biosynthesis Inc.) overnight at $37^{\circ} \mathrm{C}, 21 \% \mathrm{O}_{2}$ as previously described $[15,18]$. Additional negative controls were included by incubating U266 cells in DMSO, the peptide's solvent or in the medium. After the incubation, HLA-E expression was determined by flow cytometry by staining the cells with an HLA-E antibody (clone: 3D12HLA-E, eBioscience) or with a matched isotype control, mouse IgG1 kappa (clone: P3.6.2.8.1, eBioscience). Following the induction, U266 cells were used in the CD107a assay (Figure 6) as described in the previous section.

A

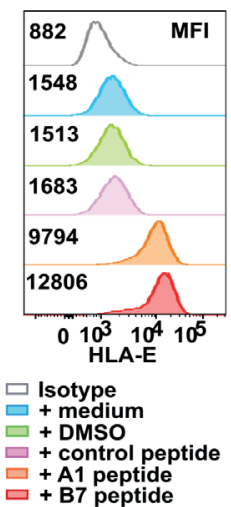

B

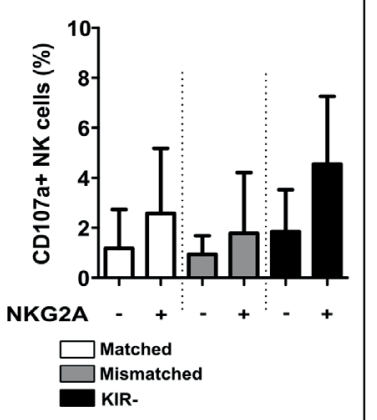

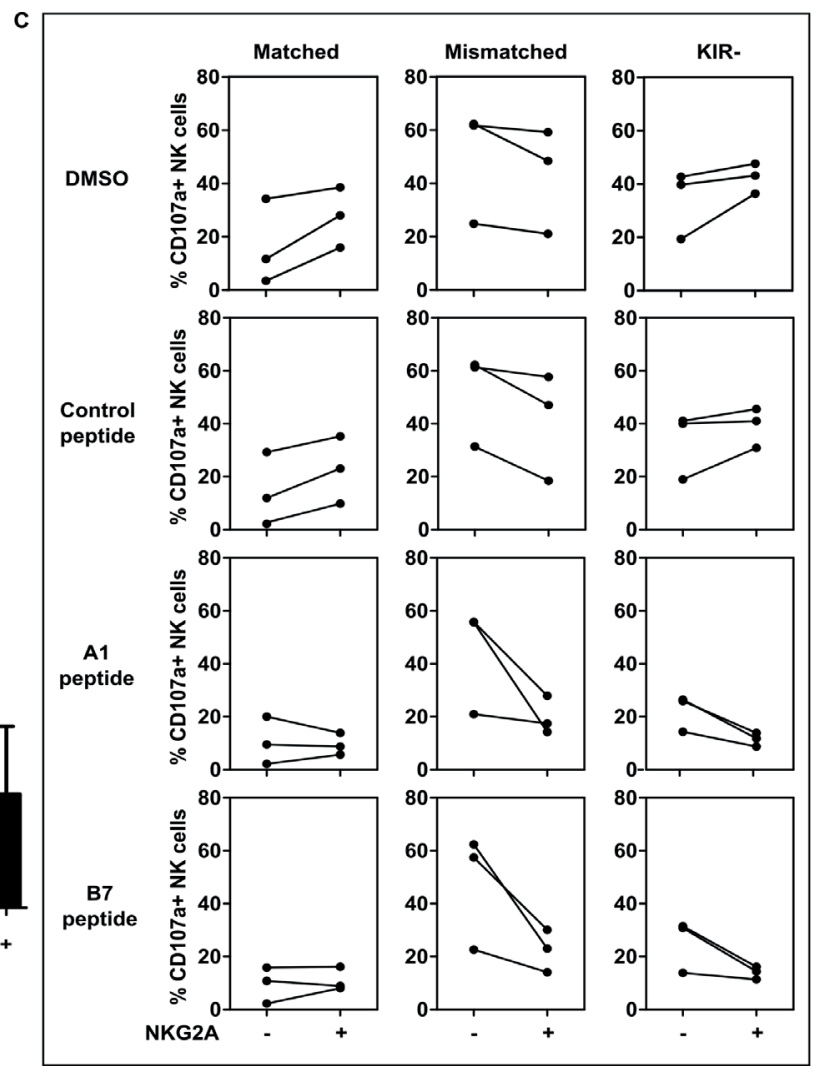

Figure 6. Inhibition via NKG2A is effective when a high level of HLA-E is present.

(A) U266 cells were pre-incubated for 2 hours with HLA-B7 peptide, HLA-A1 peptide, DMSO, control peptide (non-HLA-E binding), or medium. HLA-E expression of U266 is depicted in the histogram, with its corresponding median fluorescence intensity (MFI). (B) Spontaneous degranulation of IL-2 activated natural killer (NK) cells cultured for $13 \mathrm{~h}$ in the absence of target cells. (C) Degranulation of NK cells upon $13 \mathrm{~h}$ co-culture with peptide- or control-incubated U266 target cells. Degranulating NK cells were denoted as CD107a+ NK cells. Each dot in the graphs represents the average of a technical replicate for an individual donor. Error bars in (B) indicate standard deviation. $n=3$ different NK cell donors. 


\section{Flow cytometry}

Cells were washed with PBS (Gibco) and stained first for dead cells using Live/Dead ${ }^{\circledast}$ Fixable Aqua Dead Cell Stain Kit (Molecular Probes ${ }^{\mathrm{TM}}$, USA) for 30 minutes on ice in the dark. Cells were further washed with FACS buffer (PBS, 1\% FCS) and stained with antibodies for 30 minutes on ice in the dark. All flow cytometric analyses were performed with BD FACS Canto II. Data were analyzed with FlowJo 10.1r5 64 bit software.

\section{Statistical analysis}

All statistical analysis was performed with GraphPad Prism V software (Graphpad Software Inc, San Diego, CA, USA) using non-parametric t-test with repeated measure (Wilcoxon signed rank test. * indicates a $p$-value of $<0.05$ and $* *$ indicates a $p$-value of $<0.01,{ }^{* * *}$ indicates a $p$-value of $<0.001$ 
NKG2A expression is not per se detrimental for the anti-multiple myeloma activity of activated natural killer cells in an in vitro system mimicking the

tumor microenvironment

\section{RESULTS}

Expression of the inhibitory NKG2A receptor could be advantageous for IL-2 activated NK cells against HLA negative tumor cells

To investigate the effect of NKG2A expression on the anti-tumor response of IL-2 activated NK cells against HLA class I negative target cells, we performed a flow cytometry-based degranulation (CD107a) assay by co-culturing NK cells and HLA class I negative K562 cells followed by staining for KIRs and NKG2A to enable NK subset analysis. Under normal laboratory conditions of $21 \% \mathrm{O} 2$, a slightly higher percentage of degranulating (CD107a+) NK cells was observed for the subsets expressing NKG2A as compared to NKG2A negative counterparts, and this was observed for both KIR+ (average increase 4.6\%) and KIR- (average increase 18\%) subsets ( $p<0.05$ and $p$ $<0.001$ respectively) (Figure $1 \mathrm{~A}$ ). As the tumor microenvironment could potentially impair cytolytic effector function of the NK cells, co-cultures were also performed in the presence of biochemical context mimicking tumor microenvironment, i.e. in the presence of $0.6 \% \mathrm{O} 2$, or in the combination of hypoxia with $50 \mathrm{mM}$ lactate or $100 \mathrm{ng} / \mathrm{ml}$ prostaglandin E2 (PGE2). At $0.6 \%$ of O2, we observed more degranulation in NKG2A expressing NK cells than for the subsets not expressing KIRs (average increase 16.3\%) (Figure 1B). However, for KIR+ subsets there was no difference in the percentage of degranulating NK cells with vs without NKG2A (average increase $4.5 \%$ ) (Figure 1B). In the conditions where $0.6 \% \mathrm{O} 2$ was combined with PGE2 (average increase $11.8 \%$ for KIR- and 3.7 for KIR+) or lactate (average increase $6.1 \%$ for KIRand $4.2 \%$ for $\mathrm{KIR}+$ ), however this did not reach significance (Figure 1C, D). In the absence of target cells, the percentage of NK cell degranulation was very low (Figure S1 in Supplementary Material). Nonetheless, we also observed a similar pattern as in conditions with target cells with slightly higher percentages of degranulating NKG2A positive NK cell subsets. Of note, in none of the donors, NKG2A expression levels by the NK cells were clearly influenced by the 4 hours co-culture of NK cells and K562 in the presence of hypoxia, lactate, or PGE2 (Figure S2 in Supplementary Material). Altogether, these results suggest that against an HLA negative tumor cell line, the presence of the NKG2A receptor, especially on KIR- subsets could be beneficial for IL-2 activated NK cells also in the presence of more suppressive microenvironmental factors, presumably because these NK cells were better licensed.

\section{NKG2A does not inhibit the response of IL-2 activated NK cells against myeloma cell lines expressing low levels of HLA-E}

The interaction between the NKG2A receptor and its ligand, HLA-E, can have an inhibitory effect on the NK cell anti-tumor capacity and could outweigh the beneficial effect of improved licensing. Therefore, we investigated the effect of 
NKG2A on IL-2 activated NK cell degranulation in response to 3 multiple myeloma cell lines (UM9, RPMI8226/s, and JJN-3) expressing both HLA class I and HLA-E (Figure S3 in Supplementary Material). Based on the HLA genotypes for classical class I of the cell lines, NK cells were divided into subsets expressing: 1) no KIRs, 2) KIRs that are KIR-ligand matched- or 3) KIRs that are mismatched for the HLA ligands on the target cells. We subsequently compared the response of NK cells (co-)expressing NKG2A vs NK cells lacking NKG2A for each of the three groups. In the absence of target cells, the percentage of degranulating NK cells was negligible (Figure S4 in Supplementary Material). We observed that for the matched and the KIR negative subsets, the percentage of degranulating NKG2A positive cells was slightly higher than degranulation of the subsets lacking NKG2A in most donors. This difference reached significance when NK cells were co-cultured with RPMI8226/s both in the presence of $21 \% \mathrm{O}_{2}$ or $0.6 \% \mathrm{O}_{2}$ (Figure 2). For NK cells expressing mismatched KIRs, we did not observe a difference between NKG2A+ vs NKG2A-cells against all cell lines. Although the three cell lines tested in this study expressed HLA-E, albeit at low levels (Figure S3 in Supplementary Material), in only 4 out of 45 samples we observed a lower percentage of degranulating NK cells in NK subsets expressing NKG2A (NKG2A+ matched; NKG2A+ mismatched; or NKG2A+KIR-) as compared to their counterparts without NKG2A (NKG2A- matched; NKG2A- mismatched; or NKG2A- KIR-) and these were all in the group of NK cells expressing mismatched KIRs. These data demonstrated that the presence of NKG2A on NK cells did not seem to have an inhibitory effect on the response of IL-2 activated NK cells against HLAclass I competent cell lines expressing low levels of HLA-E. Moreover, for the subsets expressing no- or matched KIRs the NKG2A positive cells performed even slightly better than their NKG2A negative counterparts. 
NKG2A expression is not per se detrimental for the anti-multiple myeloma activity of activated natural killer cells in an in vitro system mimicking the

tumor microenvironment

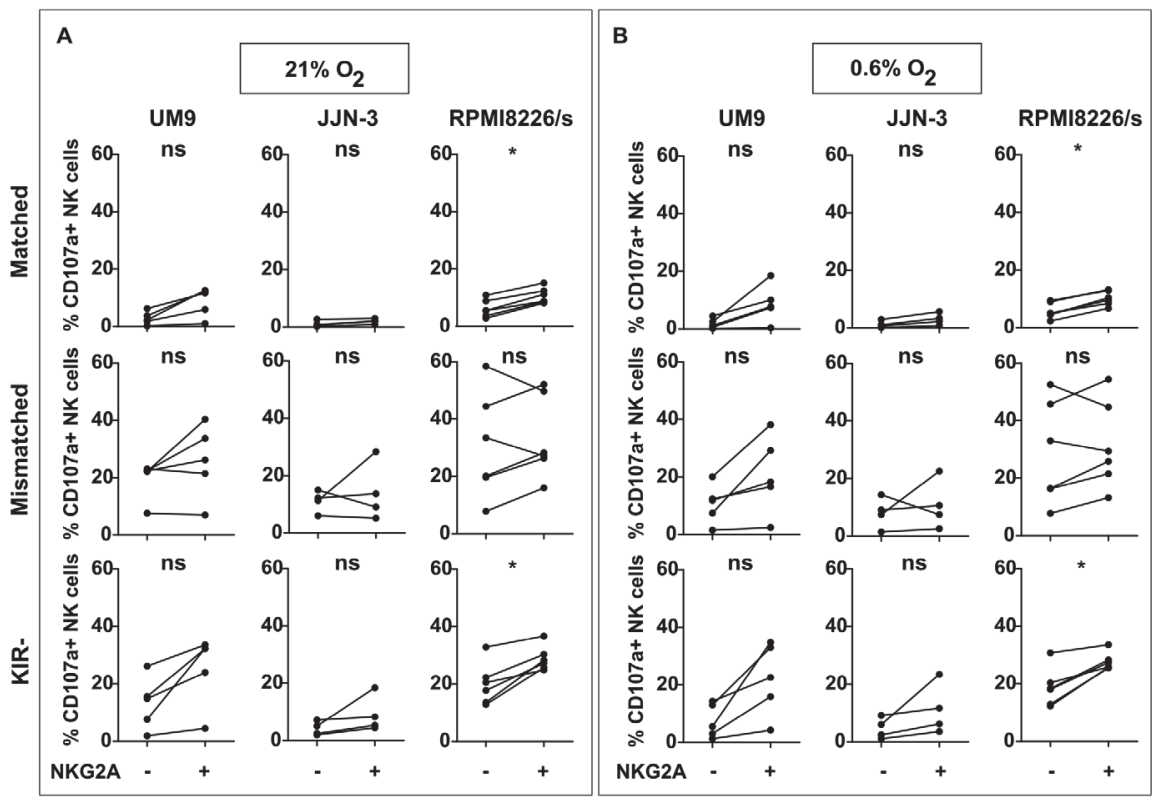

Figure 2. NKG2A and KIR subset analysis per cell line. NK cells were co-cultured with UM9, RPMI8226/s or JJN-3 cells in a 1:1 E:T ratio for 4 hours in $21 \% \mathrm{O}_{2}$ (A) or $0.6 \% \mathrm{O}_{2}$ (B). Flow cytometry was used to subtype NK cells based on their expression of NKG2A and KIRs. Degranulating NK cells were denoted as CD107a+ NK cells. Each dot represents an average of a technical replicate. $n=5$ independent experiments with 5 different donors (UM9), 4 independent experiments with 4 different donors (JJN-3) and 6 independent experiments with 6 different donors (RPMI8226/s). (ns = not significant, $\left.{ }^{*}=p<0.05\right)$

To further investigate the functional relevance of NKG2A we incubated IL-2 activated NK cells with primary MM cells known to express relatively high levels of both the classical HLA-class I and non-classical HLA-class I (HLA-E) molecules [15]. This revealed that the NK cells were highly activated by $\mathrm{K} 562$ cell line used as positive control, but not by the primary MM cells or in the absence of target cells (Figure 3A). For both KIR positive and KIR negative subsets, we did not observe any difference in NK cell degranulation between NKG2A expressing vs non-expressing NK cells both in the presence of primary MM cells (Figure $3 \mathrm{~B}$ ) or in the absence of primary MM cells (Figure S5 in Supplementary Material). As the level of degranulation in response to primary $M M$ cells was very low and this could have blunted analysis of inhibitory effects by NKG2A, we blocked the HLA-E- NKG2A interaction with an NKG2A blocking antibody (figure $3 \mathrm{C}$ ) or with an anti HLA-E antibody (Figure S6 in Supplementary Material). To study the effect in more detail, we analyzed the effect of blocking on different subsets of NK cells. However, because the blocking NKG2A antibody has the same epitope with the fluorochrome labeled NKG2A antibody, we could not visualize 
the NKG2A+ population and therefore we subtyped our NK cells into KIR+ and KIRsubtypes. Our results demonstrated that there was no effect of NKG2A blockade on the KIR+ subset and only a very small effect on the KIR- subset where we saw a small increase of CD107a+ NK cells in 2 out of 9 samples $(p<0.05)$ (Figure $3 C)$. In this analysis all KIRs were matched with the primary MM cells as the patients were $\mathrm{C} 1+$, $\mathrm{C} 2+$, Bw4+. In two samples, there was a genetic discrepancy between KIRs and HLA on the primary MM cells enabling us to subgroup NK cells based on the KIR-ligand matched/mismatched status and to investigate whether KIR-ligand interaction played a bigger role than NKG2A-HLA-E interaction in inhibiting NK cells activity. Although the number of patients was not sufficient to perform statistical analysis, this suggested that the NKG2A-KIR-ligand mismatched cells degranulated more than the NKG2A- matched counterpart (Figure 3D). NKG2A+ KIR-ligand mismatched NK cells however, were equally activated in 1 patient and more activated in 1 patient compared to the matched subset.

Altogether, the data from MM cell lines and primary MM cells demonstrated that NKG2A did not seem to play a major inhibitory role in the anti-MM response of high dose IL-2 activated NK cells.

\section{Daratumumab triggers antibody-dependent cell-mediated cytotoxicity (ADCC) in all NK cell subsets which is irrespective of the NKG2A status}

Myeloma-specific monoclonal antibodies that trigger NK cell-mediated ADCC are a potent way to boost the NK antitumor response, and also in this setting, we studied the role of NKG2A in controlling NK activation. To trigger ADCC, UM9 and RPMI8226/s, two MM cell lines that highly expressed CD38 were pre-incubated with daratumumab followed by a CD107a assay with IL-2 activated NK cells at $21 \%$ or $0.6 \% \mathrm{O}_{2}$ and analysis of degranulation of individual NK cell subsets. This revealed that the addition of daratumumab clearly triggered $\mathrm{NK}$ cell degranulation for all subsets, at $21 \% \mathrm{O}_{2}$ as well as at $0.6 \% \mathrm{O}_{2}$ (Figure S7 in Supplementary Material). For UM9, the median fold enhancement ranged from 2.5 fold to 19 fold at $21 \% \mathrm{O}_{2}$ and 2.0 fold to 44.5 fold at $0.6 \%$ $\mathrm{O}_{2}$. For RPMI8226/s, the median fold enhancement ranged from 1.9 fold to 11.4 fold at $21 \% \mathrm{O}_{2}$ and 3.5 fold to 11.6 fold at $0.6 \% \mathrm{O}_{2}$. Analysis of CD107a+ NK cells per subset subsequently demonstrated that there was no difference in degranulation between subsets expressing NKG2A vs subsets lacking NKG2A (Figure 4).

NK cells also express CD38 on their surface and previous studies showed that NK cells could kill each other via ADCC triggered by NK cell associated daratumumab. Therefore, we also compared the response of the NKG2A positive vs negative NK cells for the KIR+ and the KIR- subsets in the absence of tumor target cells. For this, IL-2 activated NK 
NKG2A expression is not per se detrimental for the anti-multiple myeloma activity of activated natural killer cells in an in vitro system mimicking the

tumor microenvironment

cells were incubated without (Figure 5A) or with daratumumab (Figure 5B-D)for four hours followed by analysis of CD107a expression by NK cell subsets at $21 \%$ or $0.6 \% \mathrm{O}_{2}$. Without daratumumab, we showed that spontaneous NK cell degranulation was very low for all subsets. For KIR+ NK cells, both at $21 \%$ and $0.6 \% \mathrm{O}_{2}$, we observed a lower percentage of degranulating NK cells in subsets co-expressing NKG2A (Figure 5B). For KIR-subsets, we only saw this in the condition at $0.6 \% \mathrm{O}_{2}$. To determine whether this was truly due to NKG2A, we blocked HLA-E-NKG2A interaction with an antibody blocking either HLA-E or NKG2A. For all donors and in both the KIR+ and KIR- NK cell subsets, the level of degranulation of NKG2A positive subsets was higher than that of NKG2A negative subsets after blocking, except in 1 donor under hypoxia in the presence of anti HLA-E, NKGA+ KIR- showed lower percentage of degranulating NK cells (Figure 5CD). This illustrates that NKG2A could inhibit daratumumab induced fratricide.

As highly activated NK cells express higher levels of HLA-E than the MM cell lines (Figure S3 in Supplementary Material), we hypothesized that the level of HLA-E might influence the potential of NKG2A to inhibit highly activated NK cells. To explore this, we performed a 4-hour degranulation assay using IL-2 activated NK cells from 3 healthy donors against U266, a multiple myeloma cell line expressing low levels of HLA-E. Prior to co-culture with NK cells, U266 cells were incubated with either medium, DMSO, control peptide, HLA-A1peptide, or HLA-B7 leader peptide. The HLA-A1 or B7 peptide are derived from the leader sequence of HLA class I and have been shown to bind HLA-E and enhance HLA-E surface expression [18]. We observed that HLA-E was highly expressed on U266 cells upon peptide incubation, approximately 6 (HLA-A1 peptide) and 8 folds (B7 peptide) higher than the baseline expression (Figure 6A). In the absence of target cells (Figure 6B), NK cells subsets expressing NKG2A showed a higher degranulation compared to NK cell subsets not expressing NKG2A. For subsets expressing matched KIRs or no KIRs, NKG2A+ NK cells degranulated more than NKG2ANK cells in the conditions where target cells were incubated without or with control peptide (Figure 6C). This was true for all three donors and in line with the data obtained with the other MM cell lines. For the KIR- subset, upregulation of HLA-E resulted in less degranulation in the NKG2A+ NK cells vs the NKG2A-cells, suggesting inhibition by NKG2A. For the matched KIR subset this effect was less pronounced. For the mismatch subset, we saw a lower percentage of degranulating NKG2A+ NK cells vs NKG2A- NK cells in all conditions. This supports the NK cell fratricide data (Figure 5) and together illustrates that NKG2A can inhibit high dose IL-2 activated NK cells but whether or not this occurs depends on the exact NK cell subset and presumably also on the type of target cells and the level of HLA-E on the target cells. 


\section{DISCUSSION}

We envision that the ideal NK cell product for cancer treatment would be a large number of highly activated natural killer cells which can withstand the suppressive tumor microenvironment. Therefore, to refine NK cell-based immunotherapy, we focus our investigations on NK cell ex vivo expansion and strategies to enhance activation and to reduce inhibition of NK cells. Since the IL-2 activated NK cell ex vivo expansion protocols could result in a higher percentage of NKG2A expressing NK cells $[19,20]$, we performed, in the current study, an in-depth analysis of the influence of NKG2A expression on different NK cell subsets in response to MM cells showing that the inhibitory potential of NKG2A, for activated NK cells, depends on the exact subset of NK cells and the HLA-E context of the target cell.

NKG2A has a dual function in NK cells, on the one hand, it is required for NK cell licensing, but it also acts as an inhibitory receptor to control the activation threshold of the NK cell and to avoid autoimmunity [3]. Here, we show that high dose IL-2 activated NK cells expressing NKG2A degranulated more vigorously than subsets not expressing NKG2A. We observed this irrespective of the presence of KIR and in response to HLA deficient $\mathrm{K} 562$ leukemia cells and to a lesser extent against HLA competent MM cell lines. For K562, this is in line with previous studies in both mice [4, 5] and human [6]. These studies showed that for unactivated NK cells, the more inhibitory receptors an NK cell expresses, the better the NK cell is licensed and the more potently it can respond to HLA-class I deficient tumors. However, for HLA competent tumors, the presence of NKG2A could be a disadvantage due to the inhibitory signaling resulting from the NKG2A-HLA-E interaction. Although some tumor cells downregulate surface expression of HLA-class I molecules, tumors can also maintain or even enhance HLAclass I [21,22]. We and others previously demonstrated that MM cell lines and primary MM cells express HLA-class I and HLA-E on their surface [15, 23]. Nevertheless, in the present study, NKG2A expression on high dose IL-2 activated NK cells did not result in a reduced activation. On the contrary, the presence of NKG2A seemed to be more advantageous for the NK cell response, especially against MM cell lines. Although NKG2A expressing vs non-expressing subsets might differ in more features than only NKG2A, our data suggest that for these high dose IL-2 activated NK cells the benefit of better licensing due to NKG2A seemed stronger than the inhibitory effects provided by this receptor.

Even in response to primary MM cells, despite relatively high HLA-E levels, no inhibitory effects of NKG2A were observed. As this could have been caused by the very low level of NK cell degranulation, we also blocked the NKG2A receptor using monoclonal 
NKG2A expression is not per se detrimental for the anti-multiple myeloma activity of activated natural killer cells in an in vitro system mimicking the

tumor microenvironment

antibodies recognized for their capacity to block HLA-E or NKG2A. The effect of blocking was very minor and only present in KIR+ subsets which was in contrast to a previous study where Monalizumab, a clinical grade NKG2A blocking antibody, improved the cytotoxicity of low dose $(250 \mathrm{U} / \mathrm{ml})$ activated KIR-NKG2A + NK cells against a variety of primary tumor cells [10]. One of the differences with our study was that Ruggeri et al used NKG2A+ KIR- NK cell clones while we used a heterogeneous population of NK cells. The percentages of NKG2A+ KIR- NK cells among our donors varied between $11.6 \%$ to $51.8 \%$ of total NK cell populations (Table S1 in Supplementary Materials) which might have influenced the blocking capacity of the antibody. In our previous study [15], also using whole NK cells but non-activated, we showed that there was an increased percentage of overall CD107a+ NK cells when we blocked HLA-E/NKG2A interaction using an HLA-E antibody. Therefore, another reason could be the difference in activation and or licensing status of the NK cells as we used in the current study healthy donorderived NK cells pre-activated with a high dose of IL-2 (1000 U/mL) while Ruggeri et al used only $250 \mathrm{U} / \mathrm{mL}$ for activation. This suggests that the activation status of the NK cells is important for whether or not NKG2A can mediate strong inhibitory effects on NK cells. Importantly this also suggests that if NK cells are sufficiently activated e.g. via cytokine activation, the co-expression of NKG2A is not per se detrimental.

The potency of NKG2A to inhibit NK cells can also be influenced by the HLA-E expression level on the target cell. Previously, inhibition via KIR has been shown to have a linear relation with HLA class I, meaning that the more of the ligand is expressed the more inhibition is mediated via the receptor [24]. For NKG2A this seems different as the same group also showed that inhibition by NKG2A occurs only when HLA-E levels are above a certain threshold and the strength of the inhibitory signal could not be further amplified by increasing expression levels of the HLA-E ligand [24]. Although the number of individuals was limited, in our experiments, peptide-induced HLA-E expression made NKG2A+KIR- less responsive than their NKG2A-KIR+ counterparts. In line with this, blockade of the NKG2A co-receptor CD94 has been shown to enhance the response of highly activated NKG2A+ NK cells against a cell line transgenically expressing very high levels of HLA-E but not against primary ALL cells expressing an intermediate level of HLA-E [25]. In addition, we observed that NKG2A positive NK cells, expressing high levels of HLA-E, mediated less daratumumab-induced fratricide than NKG2A negative NK cells which could be reversed by adding anti-HLA-E or anti-NKG2A. Highly activated T cells express increased levels of HLA-E and this protects them from killing by NKG2A positive NK cells [26]. We now show that this is also true for highly activated NK cells. Furthermore, we show that NKG2A has the potential to inhibit highly activated NK cells but that this depends on the exact setting and that activated NK cells may have a different threshold for HLA-E than unactivated NK cells. 
Another important point to take into account in the design of NK cell immunotherapy is that NK cells will have to function in a suppressive tumor microenvironment. Tumor microenvironmental factors such as hypoxia, lactate, prostaglandin E2, and others have been shown to dampen NK cell anti-tumor responses through several mechanisms summarized in [27]. We therefore evaluated the role of NKG2A also in the presence of factors from the TME but did not see very obvious differences with the data obtained under normal control conditions. In addition, we did not observe changes in NKG2A expression on the NK cells, possibly because the 4 hours incubation was relatively short to induce changes on IL-2 activated NK cells by hypoxia. We realize that our in vitro set up does not fully reflect the complexity of the in vivo TME, and several other studies in human or mice did show that the tumor microenvironmental factors could lead to NK cell phenotypic change [28-30]. Furthermore, we demonstrated in an earlier study that HLA-E levels on MM tumor cells are increased upon in vivo growth in the BM of immunodeficient mice as compared to in vitro passaged cells [15]. Moreover, a recent paper has shown that under hypoxia and glucose deprivation, HLA-E can be upregulated in both human and mouse tumor cells as a result of microenvironmental stress [31]. It would therefore be valuable to determine the effect of NKG2A on high dose IL-2 activated NK cells in an in vivo MM model or after longer exposure of hypoxia and or other tumor microenvironmental factors.

Importantly, our data show that, for high dose IL-2 activated NK cells, all subsets can get activated by tumor cells in the context of laboratory setting mimicking tumor microenvironment. This is also true for the presumed "hyporesponsive" subset not expressing any licensing inhibitory receptor. Moreover, the addition of daratumumab, to trigger $A D C C$, even improved the response of this hyporesponsive subset to a level comparable to that of subsets expressing NKG2A or KIR. This is important as it illustrates that with potent activation all subsets could contribute to tumor clearance. Nevertheless, under all conditions, subsets expressing KIRs that were mismatched with the HLA ligands on the target cell had the highest level of activation, both in response to the MM cell lines as well as in response to primary MM. This emphasizes the relevance to select KIR-ligand mismatched NK cell donors or to use a KIR blocking antibody like lirilumumab. For NKG2A, selection of mismatched donors is not a feasible strategy, but, the interaction between NKG2A and HLA-E can be blocked with a clinically available monoclonal antibody (monalizumab). However, in case of an expanded NK cell product, where NK cells received a cocktail of cytokines such as IL-2 or IL-15 providing strong activation signals, or in the situation where an ADCCtriggering antibody is used, this may not be very useful as these NK cells may be not severely inhibited by NKG2A. Additionally, blockade could even be detrimental. 
NKG2A expression is not per se detrimental for the anti-multiple myeloma

activity of activated natural killer cells in an in vitro system mimicking the

tumor microenvironment

Therefore, a better understanding of the conditions leading to HLA-E expression in relation to the inhibitory effects via NKG2A, would be useful to predict for which patients a blockade of NKG2A with monalizumab would be beneficial.

\section{CONFLICT OF INTEREST}

G. M. J. Bos is Chief Executive Officer/Chief Medical Officer/Co-founder of CiMaas, BV, Maastricht, the Netherlands. CiMaas is producing an ex vivo-expanded NK cell product that will be used to treat myeloma patients. The other authors declare no conflict of interest.

\section{AUTHORS CONTRIBUTIONS}

All authors listed have made substantial, direct, and intellectual contribution to the work and approved it for publication.

\section{FUNDING}

This study was funded by a grant from Kankeronderzoeksfonds Limburg (KOFL). L. Wieten was supported by a grant from Dutch Cancer Association (KWF kankerbestrijding; UM2012-5375).

\section{ACKNOWLEDGMENTS}

The authors would like to thank Cytogenetic laboratory, MUMC+, Maastricht, The Netherlands for processing and providing the primary MM samples and Transplantation Immunology-Tissue Typing laboratory, MUMC+, Maastricht, The Netherlands for performing the HLA genotyping for the samples. 


\section{REFERENCES}

1. Vivier E, Ugolini S, Blaise D, et al (2012) Targeting natural killer cells and natural killer T cells in cancer. NatRevImmunol 12:239-252. doi: 10.1038/nri3174

2. Thomas LM (2015) Current perspectives on natural killer cell education and tolerance: emerging roles for inhibitory receptors. Immunotargets Ther 4:45-53. doi: 10.2147/ITT.S61498

3. He Y, Tian Z (2017) NK cell education via nonclassical MHC and non-MHC ligands. Cell Mol Immunol 14:321-330. doi: 10.1038/cmi.2016.26

4. Brodin P, Lakshmikanth T, Johansson S, et al (2009) The strength of inhibitory input during education quantitatively tunes the functional responsiveness of individual natural killer cells. Blood 113:2434-2441. doi: 10.1182/blood-2008-05-156836

5. Joncker NT, Fernandez NC, Treiner E, et al (2009) NK Cell Responsiveness Is Tuned Commensurate with the Number of Inhibitory Receptors for Self-MHC Class I: The Rheostat Model. J Immunol 182:4572-4580. doi: 10.4049/jimmunol.0803900

6. Sim MJW, Stowell J, Sergeant R, et al (2016) KIR2DL3 and KIR2DL1 show similar impact on licensing of human NK cells. Eur J Immunol 46:185-191. doi: 10.1002/eji.201545757

7. Tu MM, Mahmoud AB, Makrigiannis AP (2016) Licensed and Unlicensed NK Cells: Differential Roles in Cancer and Viral Control. Front Immunol 7:166. doi: 10.3389/fimmu.2016.00166

8. Ruggeri L, Capanni M, Urbani E, et al (2002) Effectiveness of Donor Natural Killer Cell Alloreactivity in Mismatched Hematopoietic Transplants. Science (80- ) 295:2097-2100. doi: 10.1126/science. 1068440

9. Curti A, Ruggeri L, Parisi S, et al (2016) Larger size of donor alloreactive NK cell repertoire correlates with better response to NK cell immunotherapy in elderly acute myeloid leukemia patients. Clin Cancer Res 22:1914-1921. doi: 10.1158/1078-0432.CCR-15-1604

10. Ruggeri L, Urbani $E$, Andre $P$, et al (2016) Effects of anti-NKG2A antibody administration on leukemia and normal hematopoietic cells. Haematologica 101:626-633. doi: 10.3324/ haematol.2015.135301

11. Angelo LS, Banerjee PP, Monaco-Shawver L, et al (2015) Practical NK cell phenotyping and variability in healthy adults. Immunol Res 62:341-356. doi: 10.1007/s12026-015-8664-y

12. Pascal V, Schleinitz N, Brunet C, et al (2004) Comparative analysis of NK cell subset distribution in normal and lymphoproliferative disease of granular lymphocyte conditions. Eur J Immunol 34:2930-2940. doi: 10.1002/eji.200425146

13. Giebel S, Dziaczkowska J, Czerw T, et al (2010) Sequential recovery of NK cell receptor repertoire after allogeneic hematopoietic SCT. Bone Marrow Transplant 45:1022-1030. doi: 10.1038/ bmt.2009.384

14. McWilliams EM, Mele JM, Cheney C, et al (2016) Therapeutic CD94/NKG2A blockade improves natural killer cell dysfunction in chronic lymphocytic leukemia. Oncoimmunology 5:1-9. doi: 10.1080/2162402X.2016.1226720

15. Sarkar S, van Gelder M, Noort W, et al (2015) Optimal selection of natural killer cells to kill myeloma: the role of HLA-E and NKG2A. Cancer Immunol Immunother 64:951-963. doi: 10.1007/s00262-015-1694-4

16. Mahaweni NM, Bos GMJ, Mitsiades CS, et al (2018) Daratumumab augments alloreactive natural killer cell cytotoxicity towards CD38+ multiple myeloma cell lines in a biochemical context mimicking tumour microenvironment conditions. Cancer Immunol Immunother 0:0. doi: 10.1007/s00262-018-2140-1

17. Sarkar S, Germeraad WT V, Rouschop KMA, et al (2013) Hypoxia induced impairment of NK cell cytotoxicity against multiple myeloma can be overcome by IL-2 activation of the NK cells. PLoS One 8:e64835. doi: 10.1371/journal.pone.0064835 
NKG2A expression is not per se detrimental for the anti-multiple myeloma

activity of activated natural killer cells in an in vitro system mimicking the

\section{tumor microenvironment}

18. Lauterbach $\mathrm{N}$, Wieten $\mathrm{L}$, Popeijus HE, et al (2015) HLA-E regulates NKG2C+ natural killer cell function through presentation of a restricted peptide repertoire. Hum Immunol 76:578-586. doi: 10.1016/j.humimm.2015.09.003

19. Oyer JL, Igarashi RY, Kulikowski AR, et al (2015) Generation of highly cytotoxic natural killer cells for treatment of acute myelogenous leukemia using a feeder-free, particle-based approach. Biol Blood Marrow Transplant 21:632-639. doi: 10.1016/j.bbmt.2014.12.037

20. Garg TK, Szmania SM, Khan JA, et al (2012) Highly activated and expanded natural killer cells for multiple myeloma immunotherapy. Haematologica 97:1348-1356. doi: 10.3324/ haematol.2011.056747

21. Campoli M, Ferrone S (2008) HLA antigen changes in malignant cells: epigenetic mechanisms and biologic significance. Oncogene 27:5869-5885. doi: 10.1038/onc.2008.273

22. Wieten L, Mahaweni NM, Voorter CEM, et al (2014) Clinical and immunological significance of HLA-E in stem cell transplantation and cancer. Tissue Antigens 84:523-535. doi: 10.1111/ $\tan .12478$

23. Gao M, Gao L, Yang G, et al (2014) Myeloma cells resistance to NK cell lysis mainly involves an HLA class I-dependent mechanism. Acta Biochim Biophys Sin (Shanghai) 46:597-604. doi: 10.1093/abbs/gmu041

24. Cheent KS, Jamil KM, Cassidy S, et al (2013) Synergistic inhibition of natural killer cells by the nonsignaling molecule CD94. Proc Natl Acad Sci 110:16981-16986. doi: 10.1073/ pnas.1304366110

25. Björkström NK, Riese $P$, Heuts $F$, et al (2010) Expression patterns of NKG2A, KIR, and CD57 define a process of CD56dim NK cell differentiation uncoupled from NK cell education. Blood 116:3853-3864. doi: 10.1182/blood-2010-04-281675

26. Takao S, Ishikawa T, Yamashita K, Uchiyama T (2010) The rapid induction of HLA-E is essential for the survival of antigen-activated naive CD4 T cells from attack by NK cells. J Immunol 185:60316040. doi: jimmunol.1000176 [pii]\r10.4049/jimmunol.1000176

27. Baginska J, Viry E, Paggetti J, et al (2013) The Critical Role of the Tumor Microenvironment in Shaping Natural Killer Cell-Mediated Anti-Tumor Immunity. Front Immunol 4:490. doi: 10.3389/ fimmu.2013.00490

28. Mamessier $E$, Sylvain A, Thibult $M-L$, et al (2011) Human breast cancer cells enhance self tolerance by promoting evasion from NK cell antitumor immunity. J Clin Invest 121:3609-3622. doi: $10.1172 / \mathrm{JCl} 45816$

29. Krneta T, Gillgrass A, Chew M, Ashkar AA (2016) The breast tumor microenvironment alters the phenotype and function of natural killer cells. Cell Mol Immunol 13:628-639. doi: 10.1038/ cmi.2015.42

30. Pasero C, Gravis G, Guerin M, et al (2016) Inherent and Tumor-Driven Immune Tolerance in the Prostate Microenvironment Impairs Natural Killer Cell Antitumor Activity. Cancer Res 76:21532165. doi: 10.1158/0008-5472.CAN-15-1965

31. Sasaki T, Kanaseki T, Shionoya Y, et al (2016) Microenvironmental stresses induce HLA-E/Qa-1 surface expression and thereby reduce CD8+ T-cell recognition of stressed cells. Eur J Immunol 46:929-940. doi: 10.1002/eji.201545835 


\section{SUPPLEMENTARY FIGURES}

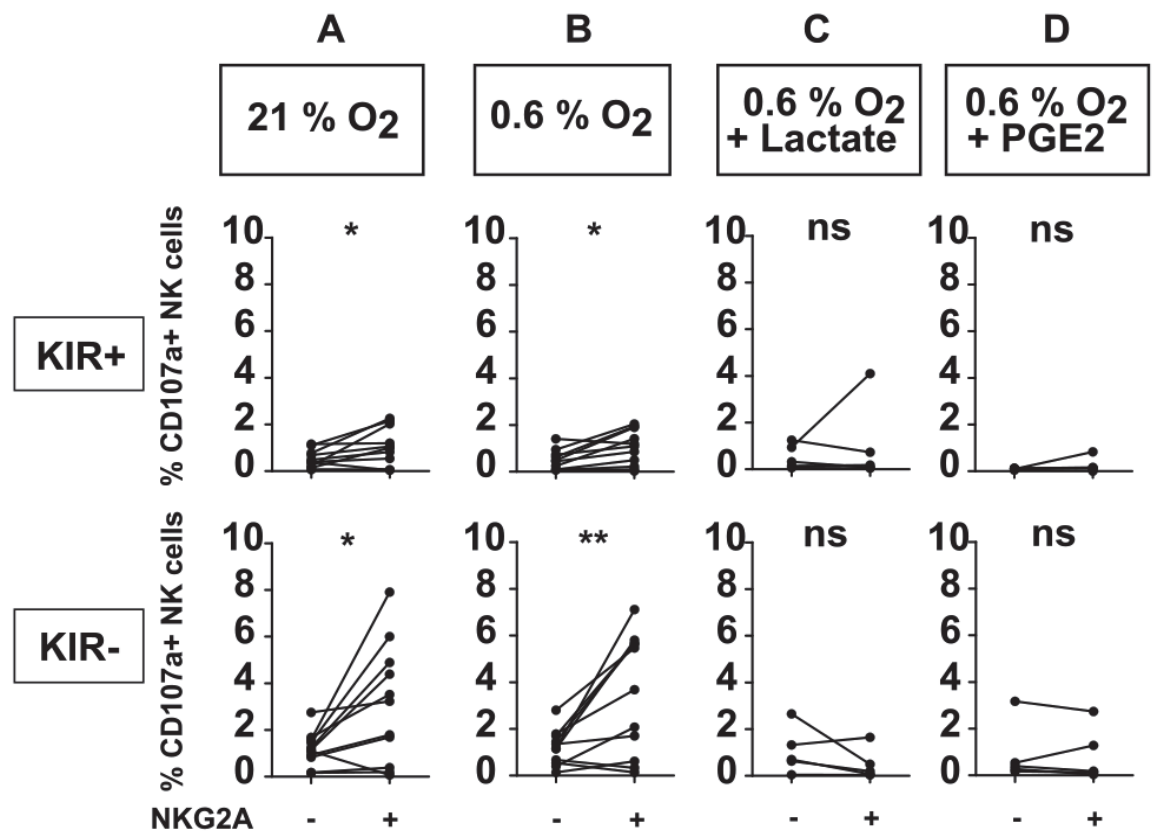

Supplementary Figure 1. Spontaneous NK cell degranulation in the presence of different microenvironment factors. NK cells were cultured without target cells for 4 hours at 21 (A) or 0.6 $\% \mathrm{O}_{2}$ (B), or combinations of $0.6 \% \mathrm{O}_{2}$ and $50 \mathrm{mM}$ lactate (C), or $100 \mathrm{ng} / \mathrm{mL}$ PGE2 (D). Flow cytometry was used to subtype NK cells based on their expression of NKG2A and KIRs. The percentage of degranulating NK cells is shown as \% CD107a+ NK cells. Each dot represents an average of a technical replicate from an individual NK cell donor. (A) and (B) $n=11$ donors, (C) and (D) $n=5$ donors tested in independent experiments $\left(^{*}=p<0.05,{ }^{* *}=p<0.01\right)$ 
NKG2A expression is not per se detrimental for the anti-multiple myeloma activity of activated natural killer cells in an in vitro system mimicking the

tumor microenvironment

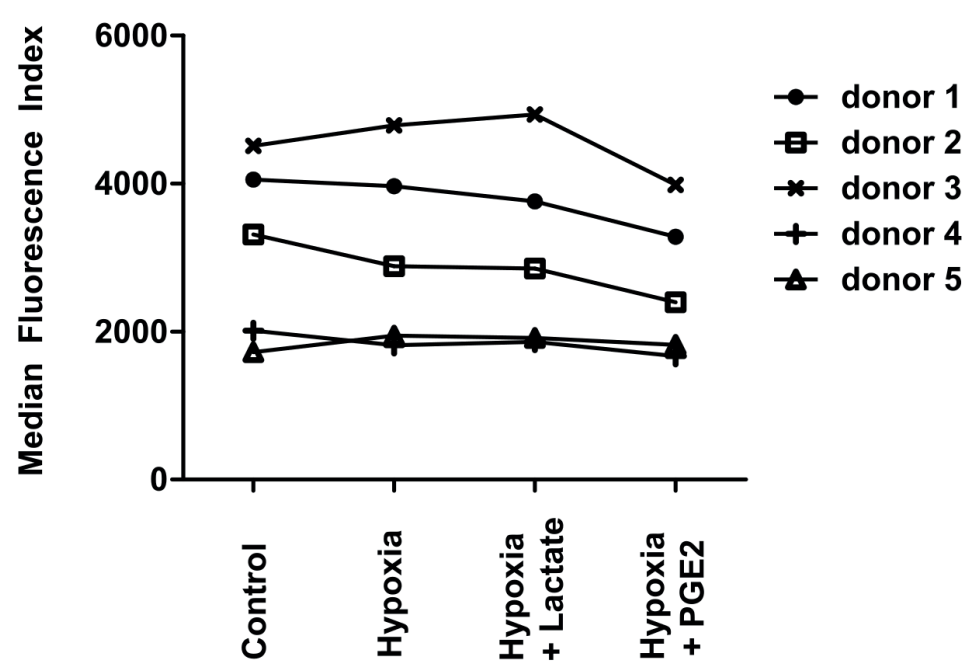

Supplementary Figure 2. NKG2A expression on NK cell is not affected by hypoxia, lactate, PGE2, or the combinations. NK cells were cultured for 4 hours in the presence of $21 \%$ or $0.6 \% \mathrm{O}_{2}$, or the combination of $0.6 \% \mathrm{O}_{2}$, and $50 \mathrm{mM}$ lactate or $100 \mathrm{ng} / \mathrm{mL}$ PGE2. Each dot represents an average of a technical replicate for the different conditions. $n=5$ independent experiments.

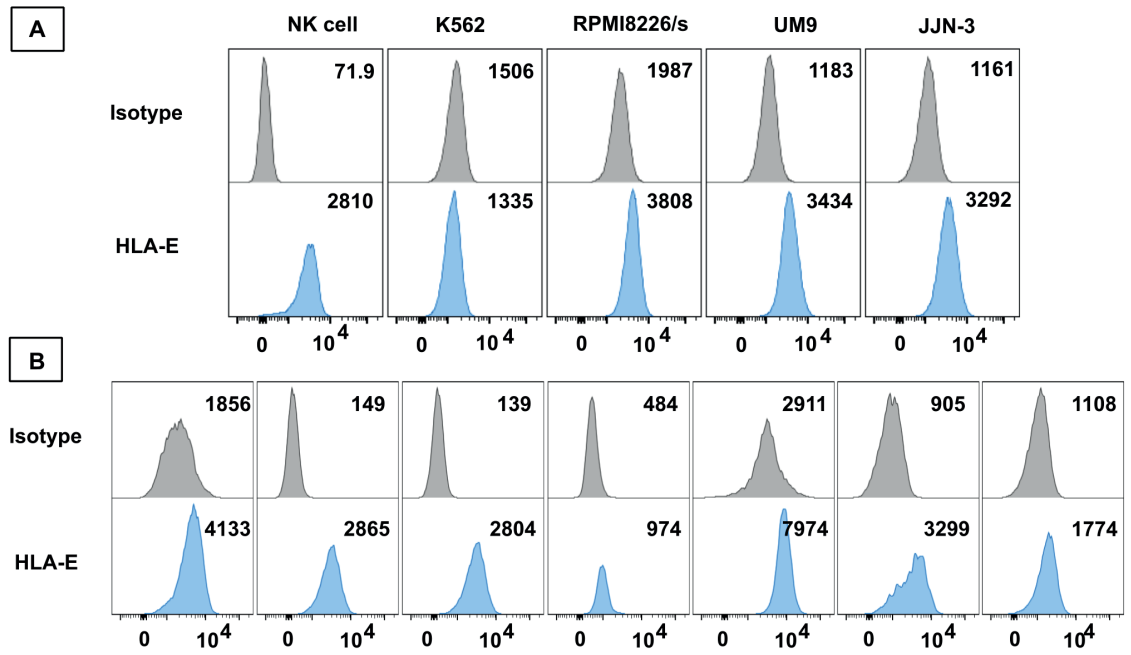

Supplementary Figure 3. HLA-E expression on different cell types. (A) IL-2 activated NK cells, K562 or MM cell lines (UM9, RPMI8226/s or JJN-3 cells) or (B) primary MM cells were stained with HLA-E antibody or isotypes as control or unstained. Each plot of primary MM cells represent 1 MM patient. Flow cytometric analysis was used to determine the expression of HLA-E. A median fluorescence intensity (MFI) is displayed in each plot. 

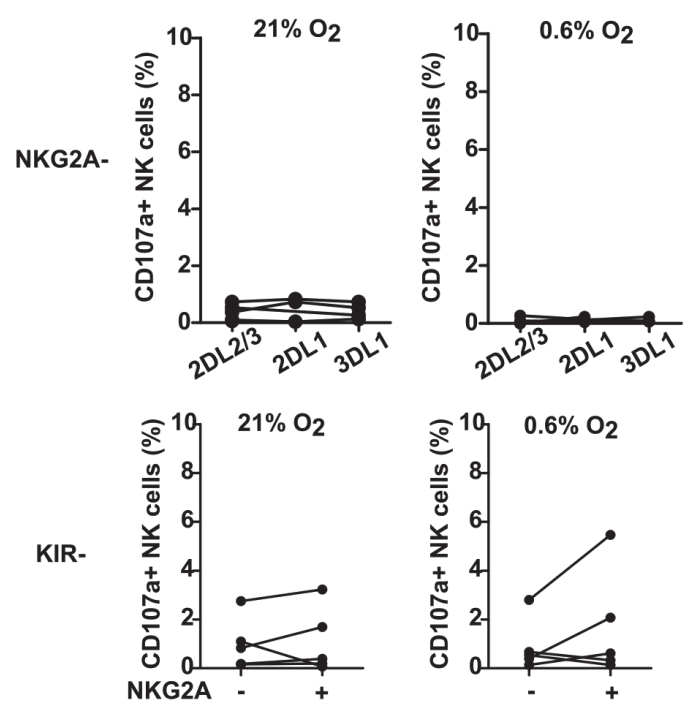

Supplementary Figure 4. Spontaneous NK cell degranulation per subset. NK cells were cultured without target cells (MM cell lines) for 4 hours in the presence of $21 \%$ or $0.6 \% \mathrm{O}_{2}$. The percentage of degranulating NK cells was denoted as CD107a+ NK cells. The subsets of 2DL2/3, 2DL1, and 3DL1 were gated based on NK cells expressing only one of the KIR receptors and lacking NKG2A expression. The subset of NKG2A+ KIR- was gated based on NK cells expressing only NKG2A receptor and missing all other KIR receptors. The subset of NKG2A- KIR- was gated based on NK cells expressing neither of NKG2A nor KIR receptors. $\mathrm{n}=5$ independent experiments.
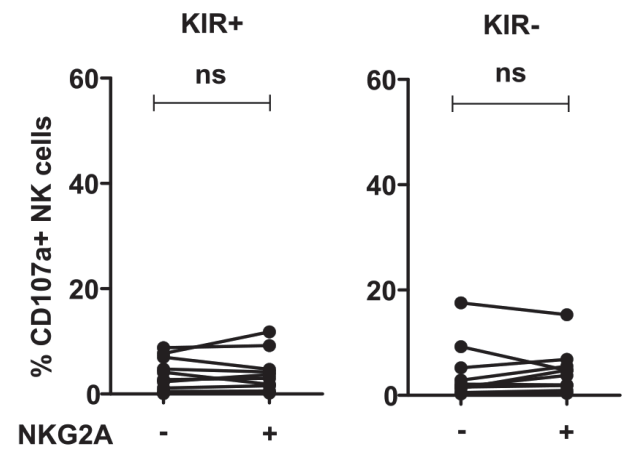

Supplementary Figure 5. Spontaneous NK cell degranulation based on NKG2A expression. NK cells were cultured without target cells (primary MM cells from patients) for 4 hours in the presence of $21 \% \mathrm{O}_{2}$. The percentage of degranulating NK cells was denoted as CD107a+ NK cells. The subset of NKG2A+ KIR- was gated based on NK cells expressing only NKG2A receptor and missing all other KIR receptors. The subset of NKG2A- KIR- was gated based on NK cells expressing neither of NKG2A nor KIR receptors.

$\mathrm{n}=10$ independent experiments 
NKG2A expression is not per se detrimental for the anti-multiple myeloma activity of activated natural killer cells in an in vitro system mimicking the

tumor microenvironment
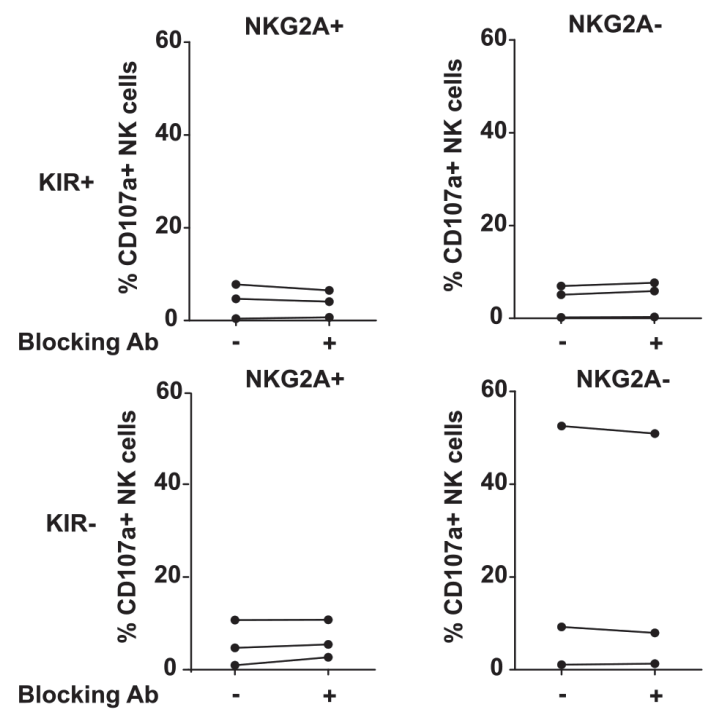

Supplementary Figure 6. Effect of HLA-E blocking on NK cell degranulation against primary MM cells. IL-2 activated NK cells were co-cultured with primary MM cells in a 1:1 E;T ratio in the presence of $10 \mu \mathrm{g} / \mathrm{mL}$ anti-HLA-E antibody for 4 hours in the presence of $21 \% \mathrm{O}_{2}$. Flow cytometric analysis was used to determine the NK cell degranulation (denoted by \%CD107a+ NK cell). $n=3$ independent experiments with three different MM patients. 


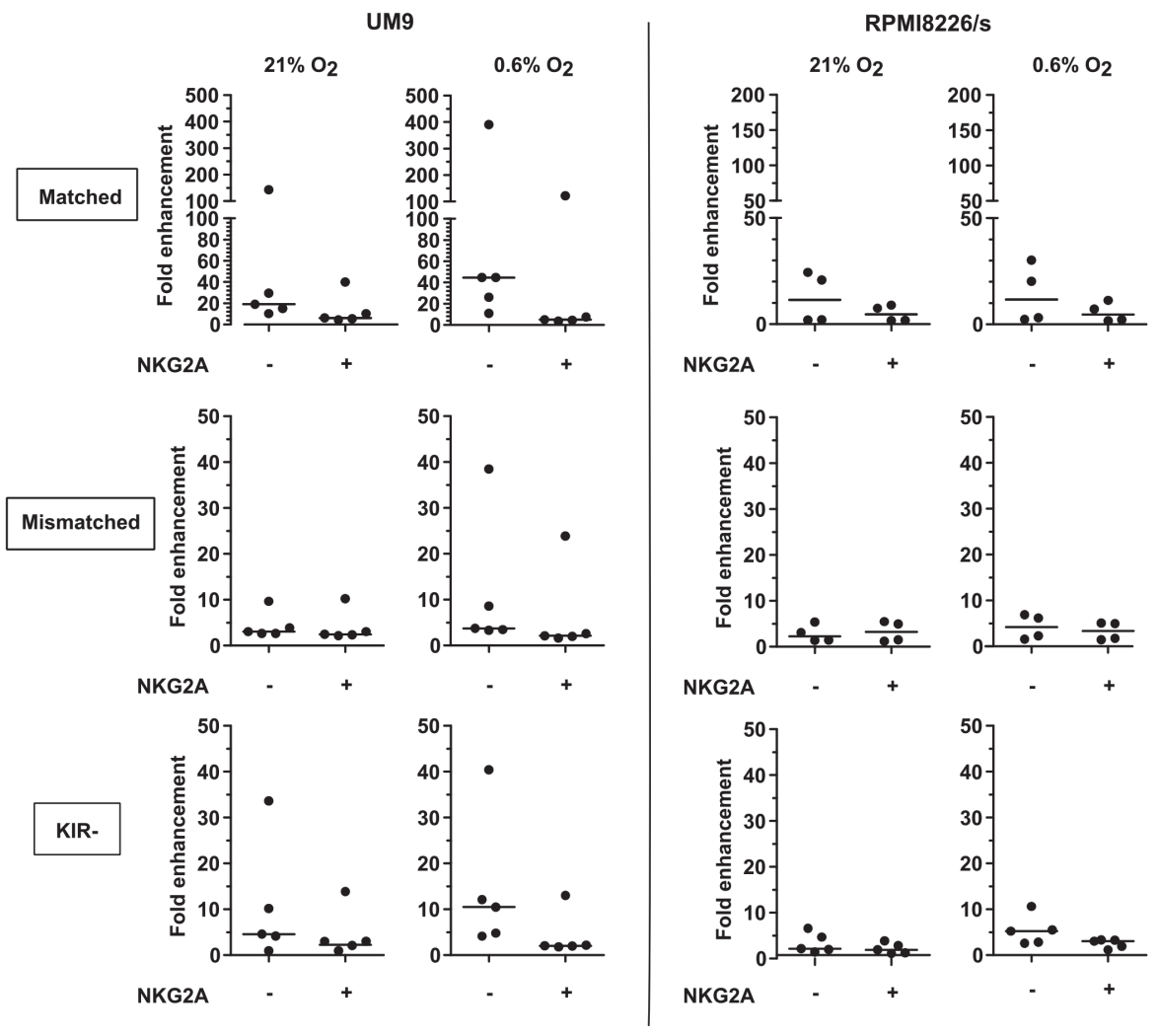

Supplementary Figure 7. Daratumumab enhanced degranulation of all NK subsets both at $21 \%$ or $0.6 \% \mathrm{O}_{2}$. UM9 or RPMI8226/s cells were pre-incubated with daratumumab or medium for 30 minutes before adding IL-2 activated NK cells in a 1:1 E:T ratio. A degranulation assay was performed for 4 hours in the presence of 21 or $0.6 \% \mathrm{O}_{2}$. Flow cytometry was used to subtype NK cells based on their expression of NKG2A and KIRs. The fold increase in the percentage of CD107a+ NK cells in the presence of daratumumab for each subset was calculated by dividing the percentage of CD107a+ NK cells in the presence of daratumumab by the percentage of CD107a+ NK cells in the absence of daratumumab. Vertical bars shown in the plots are the median.

$\mathrm{n}=5$ experiments with 5 donors 
NKG2A expression is not per se detrimental for the anti-multiple myeloma activity of activated natural killer cells in an in vitro system mimicking the

tumor microenvironment

\section{SUPPLEMENTARY TABLE}

Supplementary Table 1. The proportion of NKG2A+ KIR- cells and NKG2A- KIR-cells from different donors.

\begin{tabular}{|c|c|c|c|c|}
\hline \multirow[t]{2}{*}{ Donor } & \multicolumn{2}{|c|}{ NKG2A+ (\%) } & \multicolumn{2}{|c|}{ NKG2A- (\%) } \\
\hline & Total NKG2A+ & NKG2A+KIR- & Total NKG2A- & NKG2A- KIR- \\
\hline A & 42.9 & 30.3 & 54.4 & 14.5 \\
\hline B & 29.4 & 16.1 & 68.0 & 15.4 \\
\hline$C$ & 81.4 & 51.8 & 16.7 & 5.3 \\
\hline D & 27.7 & 18.4 & 62.4 & 26.2 \\
\hline$E$ & 54.3 & 42.5 & 37.9 & 18.1 \\
\hline $\mathrm{F}$ & 42.8 & 31.7 & 49.8 & 35.2 \\
\hline$G$ & 54.7 & 18.9 & 39.1 & 4.5 \\
\hline H & 52.1 & 34.3 & 33.6 & 13.4 \\
\hline 1 & 66.2 & 36.6 & 29.3 & 3.1 \\
\hline J & 19.2 & 11.6 & 77.8 & 14.0 \\
\hline
\end{tabular}

NK cells were stained with fluorochrome-labeled antibodies targeting NKG2A, KIR2DL2/3, KIR2DL1, and KIR3DL1 receptor. The expression of receptors was measured by flow cytometry. The percentages presented in the table were calculated from total NK cells (CD3-CD56+). $\mathrm{n}=10$ donors 
146 Chapter 6 


\section{Tuning natural killer cell anti-myeloma} reactivity by targeting inhibitory signaling via classical HLA class I and HLA-E

Parts of this chapter have been adapted from the general introduction (chapter 1) and general discussion (chapter 8 ) of this thesis.

Niken Mahaweni1, Femke Ehlers2, Gerard M.J. Bos1, Lotte Wieten2

1 Department of Internal Medicine, Division of Hematology, Maastricht University Medical Center+, Maastricht, The Netherlands; GROW School for Oncology and Developmental Biology, Maastricht University, Maastricht, The Netherlands 2 Department of Transplantation Immunology, Tissue Typing Laboratory, Maastricht University Medical Center+, Maastricht, The Netherlands; GROW School for Oncology and Developmental Biology, Maastricht University, Maastricht, The Netherlands

Under review (Frontiers in Immunology). Review article 


\section{ABSTRACT}

Natural killer (NK) cells are attractive candidates for allogeneic cell-based immunotherapy due to their unique combination of a potent anti-tumor effector function and good safety profile. In addition, NK cells have the unique capacity to attack tumor cells that lack expression of HLA class I. NK cells express inhibitory receptors like killer immunoglobulin-like receptor (KIR) family members and NKG2A. KIR and NKG2A receptors interact with HLA class I and HLA-E and are important for NK cells education. However, they can also provide inhibitory signals upon encountering HLA expressing target cells which can lead to reduced anti-tumor reactivity. Strategies to reduce inhibitory signaling by these receptors may therefore be useful to enhance clinical efficacy of NK cells against HLA expressing tumors. Multiple myeloma (MM) is an example of a tumor where this can be helpful as MM cells have been shown to express relatively high levels of HLA class I and HLA-E. Moreover, MM is an incurable hematological malignancy characterized by expansion of malignant plasma cells in the bone marrow and NK cells are interesting candidates for immunotherapy in MM. In the current review, we provide an overview of the functional relevance of inhibitory KIRs and NKG2A for the anti-tumor response of NK cells in MM. In addition, we discuss strategies to reduce inhibitory signaling via KIR and NKG2A to enhance clinical efficacy of allogeneic NK cells in MM. 


\section{INTRODUCTION}

Natural killer (NK) cells are innate lymphocytes equipped with a powerful cytotoxic machinery, the potential to produce cytokines and the capacity to selectively target diseased cells. NK cell activation is controlled by the integrated balance between signaling via a pleiotropy of inhibitory and activating receptors [1]. NK cells recognize diseased cells because, in contrast to normal healthy cells, virally-infected- or malignantly-transformed cells frequently express relatively high levels of ligands for activating NK receptors like NKG2D or DNAM-1 and reduced levels of human leukocyte antigen (HLA) ligands for inhibitory receptors $[2,3]$.

Over the past years, NK cells became popular candidates for immunotherapy in cancer due to their unique combination of a potent anti-tumor effector function and a very good safety profile [4]. The capacity of NK cells to discriminate healthy- from diseased cells creates the opportunity to safely use NK cells in the allogeneic setting and to maximally benefit from their anti-tumor potential while not risking development of graft versus host pathology. In the allogeneic setting, this latter feature is a great benefit over strategies using T cells. Although both T cells and NK cells exploit major histocompatibility (MHC) molecules for immune surveillance, they do it in an intrinsically different manner. T cell activation occurs upon interaction between the T-cell receptor (TCR) and a foreign MHC-peptide complex which, in an allogeneic MHC mismatched setting easily results in graft versus host disease (GVHD). NK cells, on the other hand, rather sense the absence of MHC molecules, a phenomenon called "missing-self recognition" described first in the 1990's by Karre et al [5]. Even in the absence of MHC molecules, NK cells do not attack healthy cells because for activation a sufficient level of activating signals, provided by viral- or stress proteins, is required and these signals are usually not sufficiently present on healthy cells [6].

The intrinsic potential to respond to missing-self enables NK cells to surveil tumors that have down regulated MHC class I expression as a mechanism to escape from CD8 T cells. NK cells express a variety of receptors and the receptors belonging to the killer Immunoglobulin-like receptor (KIR) family and NKG2A are used by the NK cells to interact with MHC class molecules. In the current review, we will provide an overview of the functional relevance of inhibitory KIR and NKG2A for the anti-tumor response of NK cells in an allogeneic setting. We will specifically address the role of allogeneic NK cells in multiple myeloma, a hematological malignancy characterized by expansion of malignant plasma cells in the bone marrow that still remains incurable despite the greatly improved clinical perspective due to novel immunomodulatory agents like lenalidomide and pomalidomide and the highly promising antibodies like daratumumab (anti-CD38) and 
elotuzumab (anti-CS-1/SLAMF7). Given their excellent safety and feasibility profiles, NK cells are interesting candidates to combine with these novel agents to enhance clinical efficacy and to ultimate achieve curative treatment for MM patients.

\section{Killer Immunoglobulin-like receptors (KIRs)}

The KIR family consists of activating- and inhibitory receptor family members. Activating family members are characterized by a short cytoplasmic ITAM activating signaling domain and are hence called KIRxDS. Inhibitory family members have a long and inhibitory ITIM domain and are named KIRxDL. Both the activating and the inhibitory KIRs have two (KIR2DSx or KIR2DLx) or three (KIR3DSx or KIR3DLx) extracellular immunoglobulin-like domains for ligand interaction. Classical HLA class I molecules are the most important ligands for both the activating- and the inhibitory KIRs. The best characterized inhibitory KIRs are: KIR2DL1 binding to HLA-C group 2 (C2) alleles having a lysine at position 80; KIR2DL2/3 interacting with HLA-C group 1 (C1) alleles having an asparagine at position 80 [7, 8]; KIR3DL1 binding HLA-B alleles bearing a Bw4 motif as well as HLA-A*23/24/32 [9] and KIR3DL2 that has been shown to interact with HLA-A*3/*11 [10] and HLA-F [11]. The activating KIRs KIR2DS1 and KIR2DS2 have been shown to bind with $C 2$ and $C 1$ alleles respectively and KIR2DS4 to HLA-C*05:01, HLA-C*11:02 and HLA-C*16:01 [12]. The ligands for the other KIRs (KIR2DS3, KIR2DS5, KIR3DS1 and KIR2DL5) remain elusive so far.

The genes encoding the KIRs are located in the KIR gene cluster in the leucocyte receptor region on chromosome 19, and so far, 17 different KIR haplotypes have been described (IMGT website). KIR2DS4 and KIR2DL5 are so-called framework genes and are present in all the haplotypes. Based on the additional presence/absence of the other KIRs, the haplotypes can be further grouped into haplotype-A and -B. While A haplotypes expresses only KIR2DS4 as activating KIR and eight other KIRs (KIR2DL1, KIR2DL3, KIR2DL4, KIR2DL5, KIR3DL1, KIR3DL2, KIR3DL3, KIR2DL5, KIR2DP1 and KIR3DP1), the $B$ haplotypes expresses multiple activating receptors in combination with various other KIR genes [13]. In the population, the $A$ to $B$ haplotype ratio is on average $1.8: 1$ [14]. A study comparing KIR haplotype $A$ and $B$ frequencies in MM demonstrated that there was no difference in distribution between MM patients and healthy individuals [14]. Moreover, analysis of KIR repertoires of one hundred eightytwo MM patients revealed that the genotypic presence of KIR3DS1 was associated with reduced progression-free survival after autologous SCT which was most pronounced in patients who were missing the KIR3DL1 ligand Bw4 [15]. But, further extensive studies on the influence of the KIR genetic repertoire on development and progression of $\mathrm{MM}$ are missing. 
In addition to gene content, further variation in KIR repertoires between individuals comes from the fact that the KIR genes are relatively polymorphic and expression differences can occur due to null/low/high expression allele variants and copy number variation [16]. Furthermore, KIRs are acquired in a stochastic manner leading to diversity, within one individual, in KIR receptor expression between NK cells [17]. Within the A haplotype four inhibitory KIRs, namely KIR2DL1, KIR2DL3, KIR3DL1, KIR3DL2 can be expressed. A combination of all four inhibitory KIRs is rarely found within one healthy individual $(<5 \%)$. Co-expression of three inhibitory KIRs occurs also in rather few NK cells (about $10 \%)$, while co-expression of $2 \mathrm{KIRs}$ and expression of a single KIR occurs more frequently (30\% and 35\%, respectively). Functionally immature NK cells, lacking all inhibitory KIRs, represent about 20\% [18]. While the characterization and the role of inhibitory KIRs have been rigorously studied and described, the role of activating KIRs on NK cells, on the other hand, are still limited.

\section{NKG2A}

NKG2A is an inhibitory member of the C-type lectin-like NKG2 receptor family that also comprises the inhibitory NKG2B and the activating NKG2C/E/H receptors [19]. NKG2A engages HLA-E, a non-classical HLA class I molecule that is constitutively expressed at low levels on the cell surface of virtually every cell. In contrast to the classical HLA class I molecules, HLA-E displays only very limited polymorphism and only two common protein variants are known (HLA-E*01:01 and HLA-E*01:03) [20]. These two HLA-E allelic variants differ in one amino acid at position 107 on the alpha2 domain of the HLA-E heavy chain, and HLA-E*01:01 has an Arginine at position 107 were HLA-E*01:03 has a Glycine [21]. This amino acid difference results in a higher peptide binding affinity and consequently a higher surface expression for HLA-E*01:03 vs HLA-E*01:01. NKG2A binds to both allotypes and so far, no obvious functional differences between the two HLA-E alleles have been described $[22,23]$.

NK cells of healthy individuals frequently express NKG2A $(20-80 \%)[24,25]$ NKG2A expression occurs more frequent on KIR-negative NK cells and decreases as NK cells acquire additional KIRs [18]. While the KIRs are highly polymorphic, NKG2A is very well conserved and not many polymorphisms are known $[13,26,27]$ possibly because the ligand, HLA-E, has also a very limited polymorphism.

\section{NK cell education and recognition of missing self}

Inhibitory receptors for HLA play a pivotal role in the shaping of a functional NK cell repertoire. NK cells develop from the bone marrow and acquire inhibitory receptors in a stochastic manner [28]. Mature NK cells can express no-, one- or a combination of inhibitory receptors. As the KIR and HLA genes are located on different chromosomes (KIR 
on chromosome 19 and HLA on chromosome 6) they can be inherited independently. Consequently, individuals can express KIRs for which the corresponding HLA ligand is missing. For example, an individual can express KIR3DL1 without being Bw4 positive. To warrant self-tolerance, even in the absence of a ligand, NK cells are continuously educated by their HLA environment in a process called "licensing" or "arming". Although the mechanistic basis of this process is not fully understood, it is known that the NK cell subsets expressing no inhibitory receptor or KIRs for which the HLA ligand is not endogenously expressed are hyporesponsive [29]. On the other hand, NK cells expressing KIRs that can engage HLA become more responsive [30]. Those so-called educated NK cells have been shown to hold higher density granules [31]. Moreover, they are more potent cytokine producers and killers than non-educated NK cells [32]. In addition to KIRs, NKG2A interaction with HLA-E can lead to enhanced NK responsiveness and from previous studies it known that the more inhibitory receptors an NK cell expresses, the more potent its effector function [33, 34].

\section{HLA class I and HLA-E expression in MM}

Many viruses and tumors have evolved strategies to reduce HLA expression presumably to escape from CD8 T cell immunity and educated NK cells are excellent in targeting these cells. However, while loss of expression of classical HLA class I is frequently seen in many types of cancer it is becoming more and more clear that numerous of these tumors remain positive for HLA-E [35]. By doing so these cells can evade from CD8 $T$ cells and, as the majority of the NK cells expresses NKG2A, the tumor also remains relatively protected against NK cells. Under physiological conditions, HLA-E expression is tightly linked to HLA class I expression. The reason is that HLA-E presents the leader peptides that are removed from HLA class I molecules before leaving the endoplasmic reticulum to travel to the cell surface [36]. Consequently, a reduced expression of HLA class I, as seen in many tumors, would also result in lower levels of HLA-E on the cell surface. But, apparently this is not necessarily the case, and tumors, as well as several viruses, have evolved ways to maintain HLA-E expression even in the absence of HLA class I leader peptides. Why this is possible is not completely known. One option could be that HLA-E presents a TAP- and HLA class I independent peptide repertoire, which has been shown to occur in TAP deficient LCL 721.221 cells [37]. Alternatively, peptides from stress proteins like Hsp60 have been shown to be capable of stabilizing HLA-E on the cell membrane [38]. Independent of the exact mechanism, it seems reasonable to consider HLA-E an immune escape mechanism for NK cells.

In MM, we observed that MM cell lines frequently express only low levels of HLA-E in vitro while HLA-E expression upon in vivo growth of the cells in the bone marrow of immunodeficient cells was much higher [39]. Moreover, primary MM cells obtained from 
patients expressed relatively high levels of HLA class I as well as HLA-E [39]. Furthermore, HLA expression has been shown to be related to disease status in MM, since MM cells isolated from late-stage pleural effusions expressed higher levels of HLA class I and reduced levels of activating NKG2D ligands as compared to earlier stage MM cells [40]. A comparable observation was made by comparing MGUS vs MM samples, showing higher levels of HLA class I and reduced levels of MICA on the MM samples [41]. Given the presence of both HLA class I and HLA-E on MM, interfering with inhibitory signaling to lower the NK cell activation threshold, so basically creating missing-self, for NK cells in MM seems a good strategy. This could be perceived either by KIR-ligand mismatching based on genotypes, the use of clinically available monoclonal antibodies to block KIR (lirilumumab) or NKG2A (monalizumab) $[42,43]$, or by agents such as the proteasome inhibitors lactacystin, bortezomib and carfilzomib that have been shown to reduce HLA class I expression in MM [44-46]

\section{Creating missing-self for MM by KIR-ligand mismatching in the allo-SCT setting}

The potential of exploiting missing-self recognition to enhance the anti-tumor potential of NK cells in the allogeneic setting became most evident from the ground-breaking work of Ruggeri et al showing that a so-called KIR-ligand mismatch improved clinical outcome after haploidentical stem cell transplantation in patients with acute myeloid leukemia (AML) $[47,48]$. In the Haplo-SCT setting, patient and donor are matched based on only one of the HLA haplotypes meaning that half of the HLA genes is mismatched between patient and donor. This enables incompatibility between KIRs, expressed on the donor NK cells, and their HLA ligands on patient tumor cells. As the donor KIR-ligand mismatched NK cells in this setting will remain to be educated based on the donor HLA background, they can efficiently detect missing-self and mediate more potent responses against the tumor cells than the non-mismatched NK cells that receive inhibitory signals via HLA (Figure 1). Only very limited data on the potential benefit of KIR-ligand mismatching in allo-SCT in MM is available. Nevertheless, Kroger et al showed that in HLA-C mismatched unrelated SCT, patients receiving a KIR-ligand mismatched graft had longer progression-free survival than patients receiving a matched graft [49]. Also in other hematological malignancies, there is still no real consensus on whether or not a KIR-ligand mismatch has a clinical benefit and presumably this is highly dependent on the exact conditioning- and transplantation protocol. In contrast to KIR-HLA class I, mismatching for HLA-E and NKG2A is not an option due to the limited polymorphism of HLA-E. However, early upon reconstitution, the relatively immature NK cells express NKG2A but not KIRs and it can take up to 3 months till a fully mature KIR repertoire is present [50]. As NKG2A could inhibit the anti-MM response of these reconstituting NK cells [39], it may be an interesting option to interfere with HLA-E NKG2A interaction using a monoclonal antibody like monalizumab in the context of allo-SCT. 
A

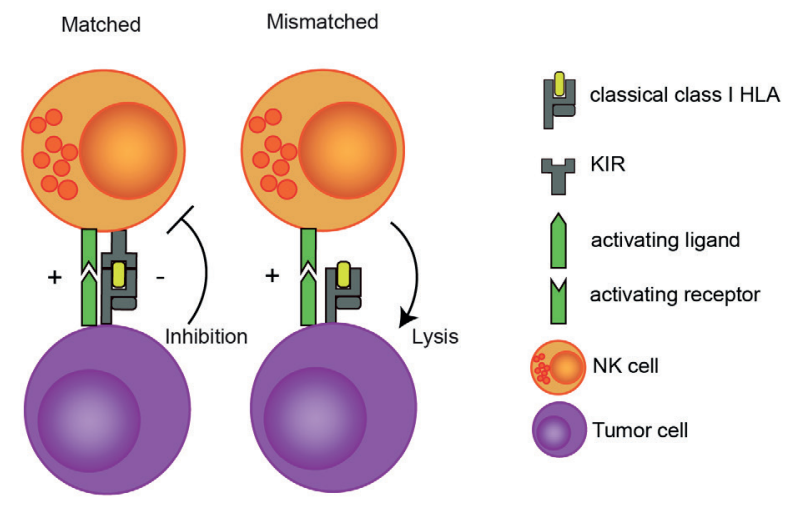

B
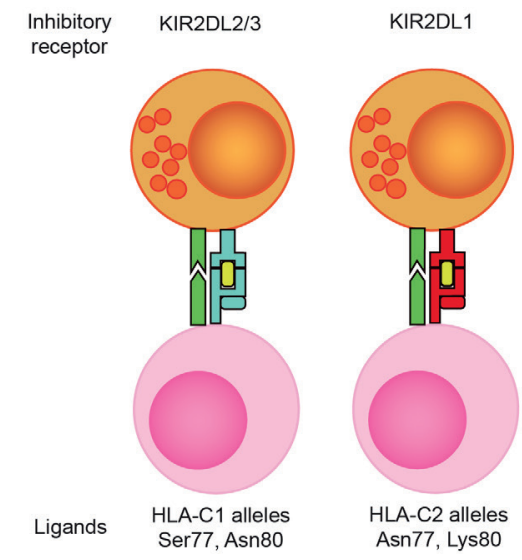

KIR3DL1

NKG2A

Asn77, Lys80
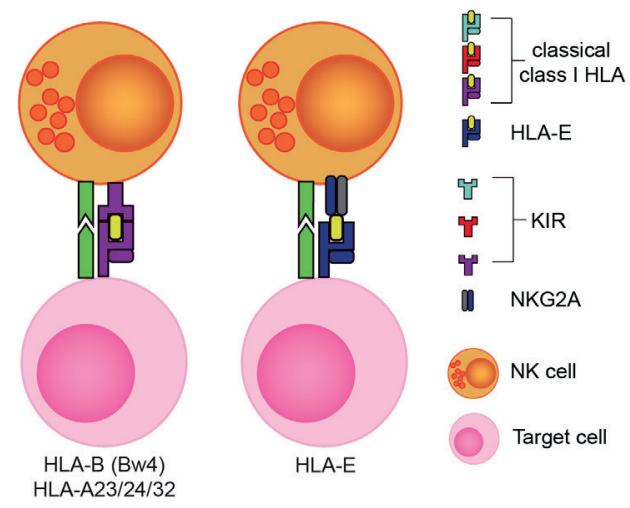

Figure 1. The concept of NK cell alloreactivity concept based on interaction with HLA class I.

A) When an inhibitory KIR binds to a "matched" classical class I HLA molecule, an NK cell receives inhibitory signal from this interaction. In the absence of the corresponding class I HLA molecule (mismatched situation), the inhibitory signal is absent, resulting in a reduced NK cell activation threshold. B) Inhibitory KIRs and NKG2A and their corresponding class I HLA molecules. KIR = Killer immunoglobulin-like receptor, HLA = Human Leukocyte Antigen, Ser = Serine, Asn = Aspargine, Lys = Lysine.

For a long time, the number of haplo-SCT that was performed was very limited due to the high occurrence of post-transplant complications such as GVHD and infections. However, due to the recent successes of improved T cell depletion methods (e.g. by ab-depletion) or by post-transplant administration of cyclophosphamide, haplo-SCT became a feasible approach with a good safety profile and the major advantage that a large number of donors is usually available within the family [51]. To be eligible 
for KIR-ligand mismatched transplantation, patients should genotypically lack expression of at least one of the HLA ligands for the major inhibitory KIRs (KIR2DL1, KIR2DL2/3, KIR3DL1), meaning that they should miss either HLA-C1, -C2 or -Bw4 or a combination thereof. We performed a small pilot study to evaluate the percentage of MM patients in our center that would meet this criterion. This revealed that there was no obvious difference between MM patients and the cohort of healthy donors that we tested (table 1). Moreover, $30 \%$ of the patients expressed all three ligands. The rest of the patients lacked at least one ligand and $48 \%$ missed even two of the ligands. In addition, to the absence of $\mathrm{C} 1, \mathrm{C} 2$ or Bw4 in the patient, the selected donor should express the HLA ligand that is missing in the patient to make sure the NK cells will be educated to sense the missing ligand and the corresponding KIR should be present on the cell surface. Especially for KIR3DL1 this is important to confirm, preferably by flow cytometry, as null alleles frequently occur [52].

Table 1. Distribution of genotypic expression of HLA epitopes in MM patients and in reference samples

\begin{tabular}{lllllll}
\hline \multicolumn{7}{c}{ HLA epitopes } \\
& $\mathrm{C} 1+$ & $\mathrm{C} 2+$ & $\mathrm{C} 1+\mathrm{C} 2+$ & Bw4+C1+ & Bw4+C2+ & Bw4+C1+C2+ \\
\hline $\begin{array}{l}\text { Patients (\%) } \\
\mathrm{n}=186\end{array}$ & 22 & 2 & 11 & 24 & 13 & 29 \\
\hline $\begin{array}{l}\text { Reference (\%) } \\
\mathrm{n}=197\end{array}$ & 28 & 2 & 15 & 16 & 17 & 32 \\
\hline
\end{tabular}

Genotypic expression of HLA-group C1, HLA-group C2 and HLA-Bw4 epitopes was determined by using sequence-specific oligonucleotides (SSO) and Luminex analysis according to manufactures instructions (One Lambda). Reference samples were obtained from healthy blood donors.

\section{Creating missing-self for NK cell adoptive transfer in MM}

As mentioned earlier, various research groups have shown that NK cells played a major role in the elimination of tumor cells in the SCT setting. Nonetheless, previous studies have also shown that NK-cell numbers and effector to target ratios' are important for tumor cell clearance $[53,54]$. Since NK cell numbers in freshly isolated NK cells or unstimulated stem cell grafts are usually low [55], optimal protocols for large scale ex vivo generation of NK cells for adoptive NK cell-based therapy have gained interest and are currently heavily investigated [56]. To date, several studies have attempted to infuse NK cells to MM patients as a form of adoptive immunotherapy. In a study by Szmania et al, up to $1 \times 10^{8}$ (per kilogram) ex vivo-expanded NK cells derived from MM patients or haploidentical family donors were infused into 8 high-risk relapsed MM 
patients [57]. In the study, NK-cell infusions were well tolerated and a significant in vivo expansion of the NK cells was observed in two subjects. Although in five patients NK-cell infusion did not affect the disease progression, in one patient it resulted in a partial response and one other patient in a delayed time to disease progression. In another study, umbilical cord blood-derived NK cells were used as a source of NK cells instead of peripheral blood-derived NK cells to infuse into twelve high-risk relapsed MM patients [58]. In this study, four different doses of NK cells were administered each to three patients; $5 \times 10^{6}, 1 \times 10^{7}, 5 \times 10^{7}$, and $1 \times 10^{8}$. This showed that that the safety profile of the NK infusions were good and that ten patients achieved a partial response as their best response. In the $21^{\text {st }}$ month of follow-up, four patients progressed or relapsed.

Although tumor cells often downregulate HLA-class I molecules to escape immunesurveillance by T-cells [59], analysis on MM cells obtained from patients showed that MM cells maintain HLA-class I expression on their cell surface $[39,40,60]$. The expression of HLA-class I on MM cells has been demonstrated as a mechanism to evade NK cell-mediated lysis [60] and selection of KIR-ligand mismatched NK cell donors could therefore be a way to enhance clinical responses of infused NK cells. As the potential benefit of a KIR-ligand mismatch is not very well established in MM we recently addressed this question in a series of in vitro studies in which we were especially interested in the functional relevance of KIR-ligand mismatching for highly activated NK cells. The reason for this was that most current insight in the role of KIR and NKG2A comes from studies using unactivated NK cells or NK cells that are reconstituting after allo-SCT and the situation might be very different for the highly activated NK cells that are typically used for adoptive NK cell therapy as their activation threshold could be changed by the activation. Our studies revealed that for unactivated NK cells as well as for highly activated ( $1000 \mathrm{U} / \mathrm{mL}$ IL-2) licensed allogeneic NK cells from healthy donors, KIR-ligand mismatched NK cells were the better effector cells compared to the KIR-ligand matched NK cells against various MM cell lines [39]. This was also the case in the presence of immunosuppressive factors like hypoxia, PGE2 and lactate that are frequently found in tumor microenvironment [61]. Even when we further potentiated the NK-cell anti-MM response via antibody-dependent cell-mediated cytotoxicity (ADCC), by combining NK-cells and daratumumab (anti-CD38), KIR ligand mismatched NK cells degranulated more robustly than their matched counterparts [61]. Although the difference between the matched and mismatched subsets was not very large, one can anticipate that in an immunosuppressive tumor microenvironment, where the NK cell receives and integrates a multitude of inhibitory signals, reduction of any extra inhibitory signals by KIR-ligand mismatching could help to potentiate the NK cell response against HLA class I competent MM cells. As many of the currently used 
ex vivo-expanded clinical NK cell products, harbor very high percentages of NKG2A positive NK cells, we also evaluated the relevance NKG2A interaction for the anti-MM response of highly activated NK cells. This showed that, at least in vitro and on ex vivo primary MM cells, the level of HLA-E on the MM cells was not sufficient to trigger potent inhibitory signaling via CD94-NKG2A [62]. Enhanced levels of HLA-E on the MM cells, by using a HLA-E stabilizing peptide, did, however, result in inhibition via NKG2A, and illustrated that the expression level of HLA-E influenced the inhibitory potential of NKG2A [62]. Together these data emphasize the complexity of the NK cell antitumor response. In addition, they suggest that for highly activated NK cells creating missing self by KIR-ligand mismatching could help to potentiate clinical efficiency while creating missing-self based on interfering with NKG2A may not be very useful given the limited inhibitory potential of NKG2A.

\section{Creating missing-self with monoclonal anti-KIR or anti-NKG2A antibodies}

In addition to creating missing self by KIR-ligand mismatching based on the donor and patient genotypes, the use of currently clinically available antibodies that block KIR or NKG2A is an interesting option to explore in MM. Blocking antibodies would especially be helpful for the $30 \%$ of donors expressing all three KIR ligands. It may also be applied under conditions where NKG2A does mediate strong inhibitory effects (e.g. for tumors with very high levels of HLA-E or for unactivated NK cells).

Blocking KIR-ligand interaction using an anti-HLA antibody showed enhanced killing of primary MM by haploidentical KIR-ligand mismatched NK cells in an in vitro autologous transplantation setting [63]. In other in vitro studies, blocking KIRs also showed promising results demonstrating that the addition of IPH2101, an anti-KIR antibody, increased NK cell cytotoxicity against HLA-C positive acute myeloid leukemia and lymphoma cells $[64,65]$. In spite of the in vitro successes, the clinical efficacy of IPH2102 still needs to be further elucidated. In a phase I clinical study in patients with relapsed/refractory MM, the IPH2101 antibody has been shown to be safe and well tolerated, however, it did not result in clear clinical responses although ex vivo patient-derived NK cells showed an enhanced cytotoxicity against MM cell line in vitro [42]. A phase II trial with IPH2101 in patients with smoldering MM, was prematurely terminated due to lack of therapeutic benefit [66]. To unravel the unexpected lack of benefit, a follow-up study was performed which showed that infusion of IPH 2101 had led to both reduced KIR2D surface expression on NK cells and reduced NK function. KIR2D removal of anti-KIR treated NK cells was mediated by trogocytosis, a mechanism by which monocytes remove antibody-bound molecules from the cell surface [67]. These studies suggest that blocking KIR by anti-KIR antibodies could 
result in uneducated, hyporesponsive NK cells and subsequently in limited effects of the antibody in vivo. Also they illustrate that, despite its in vitro potential, better understanding of how to use the blocking antibody in vivo is essential.

One way to overcome IPH2101-induced hyporesponsiveness of NK cells may be by combinational therapies in which the anti-KIR antibody IPH2101 is combined with drugs providing strong activating signals to the NK cells. In a phase I clinical trial with relapsed/refractory MM patients, the combination of the anti-KIR antibody with lenalidomide, an immunomodulatory agent, augmented NK cell function and resulted in objective responses [68]. Combination of IPH2101 and the ADCCtriggering antibody daratumumab could also enhance NK cell cytotoxicity against MM cell lines and against primary myeloma cells in vitro while IPH2101 alone did not induce a significant antitumor effect in this setting [69].

Blocking NKG2A is another option to block inhibitory NK cell signaling. In a preclinical mouse study, infusion of NKG2A+ NK cells mediated anti-leukemia effects when NK cells were pre-treated with an anti-NKG2A antibody and rescued the mice from developing leukemia [43]. In another in vitro preclinical study, blocking NKG2A with the anti-NKG2A antibody monalizumab could restore the cytotoxic potential of NK cells derived from patients with chronic lymphocytic leukemia [70]. However, thus far completed clinical trials testing safety and efficacy of monalizumab in MM patients are not available. 


\section{SUMMARY AND FUTURE PERSPECTIVE}

In contrast to several other types of cancer, MM cells have been observed to maintain or even enhance the expression of classical HLA class I molecules as well as nonclassical HLA-E. Studies with sufficient power to demonstrate a potential clinical relevance are currently lacking. Nevertheless, a limited number of, especially in vitro, studies provided evidence that creating missing self by genotypic KIR-ligand mismatching or through blocking antibodies could potentiate donor-derived NK cells in the setting of an allo-SCT or upon adoptive transfer.

To further enhance NK-cell response, novel combination strategies are currently being explored. For example, a combination with an antibody (monoclonal, bi- or tri-specific) targeting tumor-associated or -specific antigens could be used to trigger ADCC. Moreover, ex vivo NK cell expansion also provides room for further enhancement of NK-cell alloreactivity, for example by genetically manipulating the NK-cells to express a chimeric-antigen receptor (CAR). Selecting an alloreactive donor NK-cell as a source for a chimeric-antigen receptor (CAR)-NK cells could provide NKcell the KIR-ligand mismatch situation and a more effective tumor-cell recognition. Since the potential benefit of creating interfering with inhibitory HLA-induced signaling seems to depend on the activation status of the NK cell and on the input via other activating or inhibitory receptors, it will be important to test the clinical relevance of creating missing self for NK cells receiving very strong activating signal via a CAR or via potent bi- or even tri- specific antibodies.

Another recent development that is potentially relevant for MM is haplo-SCT is platform for combination immunotherapy. An interesting option it the combination of haplo-SCT and infusion of a high number of highly activated NK cells from the same donor. This combination would namely bypass the drawbacks of both of the individual procedures being 1) slow reconstitution of mature NK cells (up to 2-6 months) in Haplo SCT [71] [72] often resulting in the occurrence of infection or relapse [73] and 2) lack of persistence for ex vivo-expanded NK-cells as they are short-lived and not clonally expand like T cells do upon activation (reviewed in [74]). The combination setting would have the best of both worlds as the adoptively transferred NK cells could be manipulated during ex vivo expansion and can mediate their potent antitumor effects in the first lymphopenic period after haplo-SCT and simultaneously contribute to protection from viral infections as well. In addition, the NK cells that reconstitute from the donor stem cells will provide persistence of donor NK cells. Since haplo-SCT becomes a routine procedure in the clinic and allogeneic ex vivoexpanded NK-cell infusions have been demonstrated to be safe, combining haplo-SCT 
with an ex vivo-expanded NK-cell infusion might be a promising strategy in the future to fully exploit NK-cell alloreactivity in $70 \%$ of patients where genotypic KIR-ligand mismatch is permitting. The combination of haplo-SCT and ex vivo-expanded NK cells offers several advantages; 1 ) the process of donor selection for both procedures needs to only be done once, and 2) ex vivo-expanded NK cells could minimize the risk of infection and relapse after a haplo-SCT, as well as reduce the risk of acute GvHD. According to a recent study, the use of haplo-SCT in Europe is increasing since 2005 not only for myeloid and lymphoid malignancies, but also for solid tumors as well as non-malignant disorders [75] and also for these malignancies haplo-SCT and NK cells may be an attractive platform and also in these settings creating missing self may help to reduce the NK activation threshold and enhance clinical efficacy. At MD Anderson a clinical study using haplotransplantation with additional NK cells is underway in patients with AML. First published data [76] demonstrate that this treatment is feasible and data suggest better disease-free survival because of both an anti-disease as well as an anti-infectious effect of NK cells. In our group we recently finished a phase I study performing haplo-SCT in MM and the study is now continued as a phase II study (NL49476.000.14). This study will be the platform for haplo-SCT and NK cells. 


\section{REFERENCES}

1. Campbell KS, Colonna M (2001) Human natural killer cell receptors and signal transduction. Int Rev Immunol 20:333-370. doi: 10.3109/08830180109054413

2. Bryceson YT, Ljunggren HG (2008) Tumor cell recognition by the NK cell activating receptor NKG2D. Eur J Immunol 38:2957-2961. doi: 10.1002/eji.200838833

3. Long EO (2002) Tumor cell recognition by natural killer cells. Semin Cancer Biol 12:57-61. doi: $10.1006 /$ scbi.2001.0398

4. Dahlberg CIM, Sarhan D, Chrobok M, et al (2015) Natural killer cell-based therapies targeting cancer: Possible strategies to gain and sustain anti-tumor activity. Front Immunol. doi: 10.3389/ fimmu.2015.00605

5. Ljunggren HG, Karre K (1990) In search of the missing self:MHC molecules and NK cell recognition. ImmunolToday 11:237-244.

6. Moretta A, Biassoni R, Bottino C, et al (2000) Natural cytotoxicity receptors that trigger human NK-cell-mediated cytolysis. Immunol Today 21:228-234. doi: 10.1016/S0167-5699(00)01596-6

7. Hilton HG, Vago L, Older Aguilar AM, et al (2012) Mutation at Positively Selected Positions in the Binding Site for HLA-C Shows That KIR2DL1 Is a More Refined but Less Adaptable NK Cell Receptor Than KIR2DL3. J Immunol 189:1418-1430. doi: 10.4049/jimmunol.1100431

8. Falco M, Moretta L, Moretta A, Bottino C (2013) KIR and KIR ligand polymorphism: A new area for clinical applications? Tissue Antigens 82:363-373. doi: 10.1111/tan.12262

9. Peruzzi M, Wagtmann N, Long EO (1996) A p70 killer cell inhibitory receptor specific for several HLA-B allotypes discriminates among peptides bound to HLA-B*2705. J Exp Med 184:15851590. doi: $10.1084 /$ jem.184.4.1585

10. Döhring C, Scheidegger D, Samaridis J, et al (1996) A human killer inhibitory receptor specific for HLA-A. J Immunol 156:2-6.

11. Goodridge JP, Burian A, Lee N, Geraghty DE (2013) HLA-F and MHC Class I Open Conformers Are Ligands for NK Cell Ig-like Receptors. J Immunol 191:3553-3562. doi: 10.4049/jimmunol.1300081

12. Stewart CA, Laugier-Anfossi F, Vely F, et al (2005) Recognition of peptide-MHC class I complexes by activating killer immunoglobulin-like receptors. Proc Natl Acad Sci 102:13224-13229. doi: 10.1073/pnas.0503594102

13. Middleton D, Gonzelez F (2010) The extensive polymorphism of KIR genes. Immunology 129:819. doi: 10.1111/j.1365-2567.2009.03208.x

14. Hoteit R, Bazarbachi A, Antar A, et al (2014) KIR genotype distribution among patients with multiple myeloma: Higher prevalence of KIR 2DS4 and KIR 2DS5 genes. Meta Gene 2:730-736. doi: 10.1016/j.mgene.2014.09.008

15. Gabriel IH, Sergeant R, Szydlo R, et al (2010) Interaction between KIR3DS1 and HLA-Bw4 predicts for progression-free survival after autologous stem cell transplantation in patients with multiple myeloma. Blood 116:2033-9. doi: 10.1182/blood-2010-03-273706

16. Norman PJ, Hollenbach JA, Nemat-Gorgani N, et al (2016) Defining KIR and HLA Class I Genotypes at Highest Resolution via High-Throughput Sequencing. Am J Hum Genet 99:375-391. doi: 10.1016/j.ajhg.2016.06.023

17. Andersson S, Fauriat C, Malmberg JA, et al (2009) KIR acquisition probabilities are independent of self-HLA class I ligands and increase with cellular KIR expression. Blood 114:95-104. doi: 10.1182/blood-2008-10-184549

18. Fauriat C, Andersson S, Bjorklund AT, et al (2008) Estimation of the Size of the Alloreactive NK Cell Repertoire: Studies in Individuals Homozygous for the Group A KIR Haplotype. J Immunol 181:6010-6019. doi: 10.4049/jimmunol.181.9.6010

19. Lanier LL (2005) Nk Cell Recognition. Annu Rev Immunol 23:225-274. doi: 10.1146/annurev. immunol.23.021704.115526 
20. Kraemer T, Blasczyk R, Bade-Doeding C, et al (2014) HLA-E: A Novel Player for Histocompatibility. J Immunol Res 2014:1-7. doi: 10.1155/2014/352160

21. Strong RK, Holmes MA, Li P, et al (2003) HLA-E allelic variants: Correlating differential expression, peptide affinities, crystal structures, and thermal stabilities. J Biol Chem 278:5082-5090. doi: 10.1074/jbc.M208268200

22. Lauterbach N, Wieten L, Popeijus HE, et al (2015) Peptide-induced HLA-E expression in human PBMCs is dependent on peptide sequence and the HLA-E genotype. Tissue Antigens 85:242251. doi: $10.1111 / \tan .12525$

23. Celik AA, Kraemer T, Huyton T, et al (2016) The diversity of the HLA-E-restricted peptide repertoire explains the immunological impact of the Arg107Gly mismatch. Immunogenetics 68:29-41. doi: 10.1007/s00251-015-0880-z

24. Angelo LS, Banerjee PP, Monaco-Shawver L, et al (2015) Practical NK cell phenotyping and variability in healthy adults. Immunol Res 62:341-356. doi: 10.1007/s12026-015-8664-y

25. Pascal V, Schleinitz N, Brunet C, et al (2004) Comparative analysis of NK cell subset distribution in normal and lymphoproliferative disease of granular lymphocyte conditions. Eur J Immunol 34:2930-2940. doi: 10.1002/eji.200425146

26. Park KS, Park JH, Song YW (2008) Inhibitory NKG2A and activating NKG2D and NKG2C natural killer cell receptor genes: Susceptibility for rheumatoid arthritis. Tissue Antigens 72:342-346. doi: 10.1111/j.1399-0039.2008.01110.x

27. Iwaszko M, Świerkot J, Kolossa K, et al (2016) Influence of CD94 and NKG2A variants on susceptibility to rheumatoid arthritis and efficacy of anti-TNF treatment. Jt Bone Spine 83:7579. doi: 10.1016/j.jbspin.2015.06.010

28. Manser AR, Weinhold S, Uhrberg M (2015) Human KIR repertoires: Shaped by genetic diversity and evolution. Immunol Rev 267:178-196. doi: 10.1111/imr.12316

29. Anfossi N, André P, Guia S, et al (2006) Human NK Cell Education by Inhibitory Receptors for MHC Class I. Immunity 25:331-342. doi: 10.1016/j.immuni.2006.06.013

30. Kim S, Poursine-Laurent J, Truscott SM, et al (2005) Licensing of natural killer cells by host major histocompatibility complex class I molecules. Nature 436:709-713. doi: 10.1038/nature03847

31. Goodridge JP, Jacobs B, Saetersmoen ML, et al (2018) TRPML1-mediated Modulation of Granules Tunes Functional Potential in NK Cells.

32. Tu MM, Mahmoud AB, Makrigiannis AP (2016) Licensed and Unlicensed NK Cells: Differential Roles in Cancer and Viral Control. Front Immunol 7:166. doi: 10.3389/fimmu.2016.00166

33. Thomas LM (2015) Current perspectives on natural killer cell education and tolerance: emerging roles for inhibitory receptors. Immunotargets Ther 4:45-53. doi: 10.2147/ITT.S61498

34. Sim MJW, Stowell J, Sergeant R, et al (2016) KIR2DL3 and KIR2DL1 show similar impact on licensing of human NK cells. Eur J Immunol 46:185-191. doi: 10.1002/eji.201545757

35. Wieten L, Mahaweni NM, Voorter CEM, et al (2014) Clinical and immunological significance of HLA-E in stem cell transplantation and cancer. Tissue Antigens 84:523-535. doi: 10.1111/ $\tan .12478$

36. Iwaszko M, Bogunia-Kubik K (2011) Clinical significance of the HLA-E and CD94/NKG2 interaction. Arch Immunol Ther Exp (Warsz) 59:353-367. doi: 10.1007/s00005-011-0137-y

37. Kraemer T, Celik AA, Huyton T, et al (2015) HLA-E: Presentation of a broader peptide repertoire impacts the cellular immune response - Implications on HSCT outcome. Stem Cells Int. doi: $10.1155 / 2015 / 346714$

38. Michaëlsson J, Teixeira de Matos C, Achour A, et al (2002) A Signal Peptide Derived from hsp60 Binds HLA-E and Interferes with CD94/NKG2A Recognition. J Exp Med 196:1403-1414. doi: 10.1084/jem.20020797

39. Sarkar S, van Gelder M, Noort W, et al (2015) Optimal selection of natural killer cells to kill myeloma: the role of HLA-E and NKG2A. Cancer Immunol Immunother 64:951-963. doi: $10.1007 /$ s00262-015-1694-4 
40. Carbone E, Neri P, Mesuraca M, et al (2005) IMMUNOBIOLOGY HLA class I, NKG2D, and natural cytotoxicity receptors regulate multiple myeloma cell recognition by natural killer cells. Blood 105:251-258. doi: 10.1182/blood-2004-04-1422.Supported

41. Bernal $M$, Garrido $P$, Jiménez $P$, et al (2009) Changes in activatory and inhibitory natural killer (NK) receptors may induce progression to multiple myeloma: Implications for tumor evasion of T and NK cells. Hum Immunol 70:854-857. doi: 10.1016/j.humimm.2009.07.004

42. Benson DM, Hofmeister CC, Padmanabhan S, et al (2012) A phase 1 trial of the anti-KIR antibody IPH2101 in patients with relapsed / refractory multiple myeloma. Blood 120:4324-4333. doi: 10.1182/blood-2012-06-438028.Portions

43. Ruggeri L, Urbani $E$, Andre $P$, et al (2016) Effects of anti-NKG2A antibody administration on leukemia and normal hematopoietic cells. Haematologica 101:626-633. doi: 10.3324/ haematol.2015.135301

44. Wu X, Shao Y, Tao Y, et al (2011) Proteasome inhibitor lactacystin augments natural killer cell cytotoxicity of myeloma via downregulation of HLA class I. Biochem Biophys Res Commun 415:187-192. doi: 10.1016/j.bbrc.2011.10.057

45. Yang G, Gao M, Zhang Y, et al (2015) Carfilzomib enhances natural killer cell-mediated lysis of myeloma linked with decreasing expression of HLA class I. Oncotarget 6:26982-94. doi: 10.18632/oncotarget.4831

46. Shi J, Tricot GJ, Garg TK, et al (2008) Bortezomib down-regulates the cell-surface expression of HLA class I and enhances natural killer cell-mediated lysis of myeloma. Blood 111:1309-1317. doi: 10.1182/blood-2007-03-078535

47. Ruggeri L, Mancusi A, Capanni M, et al (2007) Donor natural killer cell allorecognition of missing self in haploidentical hematopoietic transplantation for acute myeloid leukemia: challenging its predictive value. Blood 110:433-440. doi: 10.1182/blood-2006-07-038687

48. Ruggeri L (2002) Effectiveness of Donor Natural Killer Cell Alloreactivity in Mismatched Hematopoietic Transplants. Science (80- ) 295:2097-2100. doi: 10.1126/science.1068440

49. Kröger N, Shaw B, lacobelli S, et al (2005) Comparison between antithymocyte globulin and alemtuzumab and the possible impact of KIR-ligand mismatch after dose-reduced conditioning and unrelated stem cell transplantation in patients with multiple myeloma. $\mathrm{Br} \mathrm{J}$ Haematol 129:631-643. doi: 10.1111/j.1365-2141.2005.05513.x

50. Russo A, Oliveira G, Berglund S, et al (2018) NK cell recovery after haploidentical HSCT with posttransplant cyclophosphamide: Dynamics and clinical implications. Blood 131:247-262. doi: 10.1182/blood-2017-05-780668

51. Parmesar K, Raj K (2016) Haploidentical Stem Cell Transplantation in Adult Haematological Malignancies. Adv Hematol. doi: 10.1155/2016/3905907

52. Alicata C, Pende D, Meazza R, et al (2016) Hematopoietic stem cell transplantation: Improving alloreactive Bw4 donor selection by genotyping codon 86 of KIR3DL1/S1. Eur J Immunol 46:1511-1517. doi: 10.1002/eji.201546236

53. Ilander M, Olsson-Strömberg U, Schlums H, et al (2017) Increased proportion of mature NK cells is associated with successful imatinib discontinuation in chronic myeloid leukemia. Leukemia 31:1108-1116. doi: 10.1038/leu.2016.360

54. Balsamo M, Vermi W, Parodi M, et al (2012) Melanoma cells become resistant to NK-cell-mediated killing when exposed to NK-cell numbers compatible with NK-cell infiltration in the tumor. Eur J Immunol 42:1833-1842. doi: 10.1002/eji.201142179

55. Suck G, Koh MBC (2010) Emerging natural killer cell immunotherapies: Large-scale ex vivo production of highly potent anticancer effectors. Hematol Oncol Stem Cell Ther 3:135-142. doi: 10.1016/S1658-3876(10)50024-4

56. Koehl U, Kalberer C, Spanholtz J, et al (2016) Advances in clinical NK cell studies: Donor selection, manufacturing and quality control. Oncoimmunology 5:1-11. doi: 10.1080/2162402X.2015.1115178 
57. Szmania S, Lapteva N, Garg T, et al (2015) Ex Vivo-expanded Natural Killer Cells Demonstrate Robust Proliferation In Vivo in High-risk Relapsed Multiple Myeloma Patients. J Immunother 38:24-36. doi: 10.1097/CJ.0000000000000059

58. Shah N, Li L, McCarty J, et al (2017) Phase I study of cord blood-derived natural killer cells combined with autologous stem cell transplantation in multiple myeloma. $\mathrm{Br} J$ Haematol 177:457-466. doi: 10.1111/bjh.14570

59. Hicklin DJ, Marincola FM, Ferrone S (1999) HLA class I antigen downregulation in human cancers: T-cell immunotherapy revives an old story. Mol Med Today 5:178-186. doi: 10.1016/ S1357-4310(99)01451-3

60. Gao M, Gao L, Yang G, et al (2014) Myeloma cells resistance to NK cell lysis mainly involves an HLA class I-dependent mechanism. Acta Biochim Biophys Sin (Shanghai) 46:597-604. doi: 10.1093/abbs/gmu041

61. Mahaweni NM, Bos GMJ, Mitsiades CS, et al (2018) Daratumumab augments alloreactive natural killer cell cytotoxicity towards CD38+ multiple myeloma cell lines in a biochemical context mimicking tumour microenvironment conditions. Cancer Immunol Immunother 0:0. doi: 10.1007/s00262-018-2140-1

62. Mahaweni NM, Ehlers FA, Sarkar S, et al (2018) NKG2A Expression is Not per se Detrimental for the Anti-Multiple Myeloma Activity of Activated Natural Killer Cells in an In Vitro System Mimicking the Tumor Microenvironment. Front Immunol. doi: 10.3389/fimmu.2018.01415

63. Shi J, Tricot G, Szmania S, et al (2008) Infusion of haplo-identical killer immunoglobulin-like receptor ligand mismatched NK cells for relapsed myeloma in the setting of autologous stem cell transplantation. Br J Haematol 143:641-53. doi: 10.1111/j.1365-2141.2008.07340.x

64. Romagne F, Andre P, Spee P, et al (2009) Preclinical characterization of 1-7F9, a novel human anti-KIR receptor therapeutic antibody that augments natural killer-mediated killing of tumor cells. Blood 114:2667-2677. doi: 10.1182/blood-2009-02-206532

65. Kohrt HE, Thielens A, Marabelle A, et al (2014) Anti-KIR antibody enhancement of anti-lymphoma activity of natural killer cells as monotherapy and in combination with anti-CD20 antibodies. Blood 123:678-686. doi: 10.1182/Blood-2013-08-519199

66. Korde N, Carlsten M, Lee M-J, et al (2014) A phase II trial of pan-KIR2D blockade with IPH2101 in smoldering multiple myeloma. Haematologica 99:e81-e83. doi: 10.3324/haematol.2013.103085

67. Carlsten M, Korde N, Kotecha R, et al (2016) Checkpoint inhibition of KIR2D with the monoclonal antibody IPH2101 induces contraction and hyporesponsiveness of NK cells in patients with myeloma. Clin Cancer Res 22:5211-5222. doi: 10.1158/1078-0432.CCR-16-1108

68. Benson DM, Cohen AD, Jagannath S, et al (2015) A phase I trial of the anti-KIR antibody IPH2101 and lenalidomide in patients with relapsed/refractory multiple myeloma. Clin Cancer Res 21:4055-4061. doi: 10.1158/1078-0432.CCR-15-0304

69. Nijhof IS, Van Bueren JJL, Van Kessel B, et al (2015) Daratumumab-Mediated lysis of primary multiple myeloma cells is enhanced in combination with the human Anti-KIR antibody IPH2102 and lenalidomide. Haematologica 100:263-268. doi: 10.3324/haematol.2014.117531

70. McWilliams EM, Mele JM, Cheney C, et al (2016) Therapeutic CD94/NKG2A blockade improves natural killer cell dysfunction in chronic lymphocytic leukemia. Oncoimmunology 5:1-9. doi: 10.1080/2162402X.2016.1226720

71. Nguyen S, Dhedin N, Vernant JP, et al (2005) NK-cell reconstitution after haploidentical hematopoietic stem-cell transplantations: Immaturity of NK cells and inhibitory effect of NKG2A override GvL effect. Blood 105:4135-4142. doi: 10.1182/blood-2004-10-4113

72. Rizzieri DA, Koh LP, Long GD, et al (2007) Partially Matched, Nonmyeloablative Allogeneic Transplantation: Clinical Outcomes and Immune Reconstitution. J Clin Oncol 25:690-697. doi: 10.1200/JCO.2006.07.0953 
73. Chang YJ, Zhao XY, Huang XJ (2014) Immune reconstitution after haploidentical hematopoietic stem cell transplantation. Biol Blood Marrow Transplant 20:440-449. doi: 10.1016/j. bbmt.2013.11.028

74. Martín-Antonio B, Suñe G, Perez-Amill L, et al (2017) Natural killer cells: Angels and devils for immunotherapy. Int J Mol Sci. doi: 10.3390/ijms18091868

75. Passweg JR, Baldomero H, Bader $\mathrm{P}$, et al (2017) Use of haploidentical stem cell transplantation continues to increase: The 2015 European Society for Blood and Marrow Transplant activity survey report. Bone Marrow Transplant 52:811-817. doi: 10.1038/bmt.2017.34

76. Ciurea SO, Schafer JR, Bassett R, et al (2017) Phase 1 clinical trial using mbIL21 ex vivo expanded donor-derived NK cells after haploidentical transplantation. Blood 130:1857-1869. doi: 10.1182/blood-2017-05-785659. 
166 | Chapter 7 


\section{Less is more: a low glucose} concentration during short and long term cultures is correlated with a better antitumor response and viability of activated NK cells

Niken M. Mahaweni1,2, Birgit L. M. G Gijsbers1, Marcel G. J. Tilanus2, Gerard M. J. Bos1, Lotte Wieten2

1 Department of Internal Medicine, Division Hematology, Tumor Immunology Laboratory, GROW School for Oncology and Developmental Biology, Maastricht University Medical Center+, Maastricht, The Netherlands

2 Department of Transplantation Immunology, Tissue Typing Laboratory, GROW School for Oncology and Developmental Biology, Maastricht University Medical Center+, Maastricht, The Netherlands

In preparation 


\section{ABSTRACT}

Tumor cells rely mostly on the aerobic glycolysis resulting in a decreased glucose availability in the TME of solid tumors and metabolic competition with immune cells rendering dysfunctional immune surveillance. To date, glucose levels in the hematological malignancies and the effect of low glucose concentration on highly activated NK cells are largely unknown. In the current study, we compared glucose levels in the bone marrow (BM) of patients with multiple myeloma (MM) vs healthy donors and tested their effect on highly activated NK cells.

Glucose from BM samples from MM patients and healthy donors (each $n=9$ ) were measured using a biochemical analyzer and found to be lower in MM patients (479 to $1231 \mathrm{mg} / \mathrm{L}$; mean $=731.8 \mathrm{mg} / \mathrm{L}, \mathrm{SD}=247.6)$ than in in healthy donors $(2297$ $4196 \mathrm{mg} / \mathrm{L}$; mean $=3337 \mathrm{mg} / \mathrm{L}, \mathrm{SD}=661.5$ ). To test the effect of glucose on NK-cell cytotoxicity and viability, NK cells were co-cultured at a 1:1 E:T ratio with K562 cells in a 4-hour cytotoxicity assay after a short-term culture in the presence of $1000 \mathrm{U} /$ $\mathrm{mL}$ IL-2 or after a two week NK-cell expansion protocol followed by 4 days exposure to different glucose levels. Low glucose concentration (comparable to MM BM glucose levels) during the 4-hour cytotoxicity assay did not negatively affect NK cell cytotoxicity. However, higher glucose concentrations (comparable to normal glucose BM or in vitro concentrations) diminished NK cell cytotoxicity against K562 cells. Longer exposure $(\mathrm{o} / \mathrm{n})$ to low glucose concentrations did not reduce NK cell cytotoxicity against K562 cells and conferred a better survival for NK cells during the 4-hour cytotoxicity assay. NK cells cultured for 4 days in the presence of low glucose concentrations were better capable of killing K562 cells and were less susceptible to die during the 4-hour cytotoxicity assay.

In summary, we showed that a low glucose concentration during short- (overnight) or longer- term culture (4 days) did not compromise cytotoxicity or viability of highly activated NK-cells. On the contrary, our data showed that low glucose concentration might even be favorable during culture, activation and killing process. 


\section{INTRODUCTION}

In the last decade, considerable effort has been put in the development of NK cellbased immunotherapy to treat cancer patients due to the NK cells clinical potential and good safety profile. Multiple clinical trials using either autologous or allogeneic NK cells in various types of hematological- and solid cancers have demonstrated that NK cells could exert antitumor responses in patients without significant toxicity [1-3]. Nonetheless, despite these initially hopeful clinical outcomes, the therapeutic efficacy of NK cell-based immunotherapy remains limited and the outcome of NK cell-based immunotherapy should be improved by increasing NK-cell numbers, enhancing NKcell activation, improving NK-cell tumor-targeting capacity, and improving in vivo NK-cell persistence [1].

The expansion and persistence of NK cells in vivo has been demonstrated to be positively correlated with the clearance of leukemic cells in patients receiving adoptive NK cell therapy [4]. However, to be able to survive in the tumor microenvironment (TME), NK cells require cytokines such as IL-2 or IL-15. Although IL-2 or IL-15 is produced by several cell types which might present in the TME, the amount available might not be enough [5]. In classic Hodgkin Lymphoma, for example, IL-2 was scarcely found in the TME and there was a competition between the cancer cells and NK cells in utilizing IL-15 [6]. The presence of other cytokines such as transforming growth factor-beta (TGF-ß) and IL-10 in the TME plays also a role in the suppression of IL-2 production [7]. Additionally, the microenvironment of tumor cells could be unfavorable and even suppressive for NK cells allowing tumor cells to escape the NK-cell antitumor response. Factors present in the tumor microenvironment such as myeloid derived suppressor cells [8], hypoxia [9, 10], or factors released by the tumor cells such as prostaglandin E2, TGF- 3, IL-10, reactive oxygen species, and arginase [11-13] have been described to hinder NK cell antitumor capacity. Additionally, the metabolic microenvironment of tumor cells could also inhibit the antitumor response of immune cells such as cytotoxic T cells and NK cells [14].

To sustain their growth and survival, tumor cells frequently undergo metabolic reprogramming, allowing the enhancement of glucose uptake and metabolism. This process takes place not only within a hypoxic region but also in the area where sufficient oxygen is available, a phenomenon known as aerobic glycolysis or "the Warburg effect" [15]. Aerobic glycolysis is favorable for proliferating cells since it can provide both bioenergetics and biosynthesis need of a proliferating cell better than oxidative phosphorylation (OxPhos) [14]. 
Due to the high rate of glycolysis, the glucose supply in the tumor microenvironment can be limited. Average glucose levels in the microenvironment of several types of solid tumors have been reported to be low, ranging between 0.1 and $0.4 \mathrm{mM} \mathrm{(18-72}$ $\mathrm{mg} / \mathrm{L})$, which is much lower than the average normal blood glucose of $6 \mathrm{mM}$ (1080 $\mathrm{mg} / \mathrm{L})$ [16]. These values have been also reported to be not uniformly distributed within the microenvironment and the glucose concentration was inversely correlated with the distance from the capillary [16]. Nonetheless, to our knowledge, there is not much known about glucose levels within the microenvironment of hematological cancers.

Aerobic glycolysis appeared to be not only advantageous for tumor cells. Immune cells, such as cytotoxic T cells, have been shown to require a switch to aerobic glycolysis to exert their effector function [17]. Since both tumor cells and T cells are glycolytic, metabolic competition can occur within the tumor microenvironment. The glycolytic activity of the tumor cells can cause depletion of extracellular glucose thereby limiting the availability of glucose to T cells [18]. In mice, it has been demonstrated that this metabolic competition hindered T-cell metabolism resulting in a defective IFN- $\gamma$ production which is crucial for antitumor response [19].

In NK cells, aerobic glycolysis has also been shown to be important for a potent NKcell effector function. In mice, resting NK cells preferred OxPhos for their metabolism while highly activated NK cells enhanced especially glycolysis and, to a lower extend OxPhos [20, 21] [22]. In humans, NK cells upregulated both glycolysis and OxPhos upon cytokine stimulation with IL-2 or IL-12/15 [23]. Additionally, CD56 bright NK cells were found to be metabolically more active than CD56 dim NK cells [23] . The same group also showed that elevated levels of OxPhos were essential for NK cell effector cytotoxicity and IFN- $\gamma$ production. Furthermore, they showed that although the increase in glycolysis was not directly required for NK cell degranulation, restricting the rate of glycolysis resulted in a defected IFN- $\gamma$ production by CD56 bright NK cells.

Our group focuses on the development and refinement of NK cell-based immunotherapy to treat patients with cancer, especially multiple myeloma (MM) as there is no cure available to date for MM. To do this, we envision to inject a high number of highly activated NK cells to patients with MM. Since glucose levels in the MM microenvironment, as well as the effect of potentially low levels of glucose on the antitumor capacity of highly activated cytotoxic (CD56 dim) NK cells remain unknown, we aimed to explore the possible consequences of $\mathrm{MM}$ metabolic microenvironment on NK cell antitumor potential. First, we investigated the levels of glucose present in the microenvironment of patients having active MM to define relevant in vitro 
experimental conditions. Second, based on these results, we performed in vitro 4-hour cytotoxicity assays to study the effect of short-term exposure to different glucose concentrations on NK cell cytotoxicity against tumor cells and on NK cell viability. Third, we studied the influence of longer-term exposure of different glucose concentrations on expanded NK cells to evaluate whether NK cell effector function could be optimized by adapting glucose levels during expansion. The results from this current study would give us more insight whether in vivo glucose concentration should be a concern for the NK cell-based immunotherapy and whether eventually an intervention might be needed to improve the therapy. 


\section{Materials and Methods}

\section{Cell lines and culture}

The K562 cell line, purchased from American Type Culture Collection (ATCC, USA), was cultured in IMDM (Gibco, Breda, The Netherlands) supplemented with $10 \%$ fetal calf serum (FCS) (Greiner Bio-One International, Gmbh), $100 \mathrm{U} / \mathrm{mL}$ penicillin (Gibco) and 100 $\mu \mathrm{g} / \mathrm{mL}$ streptomycin (Gibco) at $37^{\circ} \mathrm{C}$ in humidified air containing $5 \% \mathrm{CO}_{2}$ with $21 \% \mathrm{O}_{2}$ (Sanyo MCO-20AIC, Sanyo Electric Co, Japan).

\section{NK cell culture and NK cell expansion}

NK cells were isolated from buffy coats by negative selection of NK cells isolation kit using MACS beads and columns according to manufacturer's protocol (Miltenyi Biotec, $\mathrm{GmbH}$ ). For experiments in Figure 2 and 3, NK cells were activated overnight with 1000 $\mathrm{IU} / \mathrm{ml}$ recombinant human IL-2 (Proleukin, Novartis) in RPMI-1640 medium (Gibco) supplemented with $10 \%$ fetal calf serum (Greiner Bio-One), $100 \mathrm{U} / \mathrm{mL}$ penicillin (Gibco) and $100 \mu \mathrm{g} / \mathrm{mL}$ streptomycin (Gibco) at $37^{\circ} \mathrm{C}$ in humidified air containing $5 \% \mathrm{CO}_{2}$ with $21 \% \mathrm{O}_{2}$ (Sanyo MCO-20AIC, Sanyo Electric Co, Japan). For experiments in Figure 4 and 5, NK cells were expanded from CD3-depleted peripheral blood mononuclear cells (PBMCs) derived from buffy coats for 17 days using 2 different expansion protocols. In protocol 1 , NK cells were expanded in the alpha-medium (Biochrom, Gmbh) supplemented with $10 \%$ human serum (Milan Analytica, AG), 2mM L-GlutaMax (Gibco), 1.3 g/L Sodium bicarbonate (Biochrom), $2000 \mathrm{mg} / \mathrm{L}$ glucose (Sigma), and 0.5\% Gentamycin-Sulphate (Gibco) in the presence of $1000 \mathrm{U}$ IL-2/mL for 17 days. In protocol 2, NK cells were expanded in SCGM medium (CellGenix) supplemented with 10\% FCS and $2 \mathrm{mM} \mathrm{L-GlutaMax} \mathrm{(Gibco)} \mathrm{in} \mathrm{the}$ presence of $100 \mathrm{U} \mathrm{IL}-2 / \mathrm{mL}$ for 17 days in combination with $200 \mu \mathrm{g}$ PM21 particles/mL added on day 0 and day 7. PM21 particles [24] were provided by Dr. Alicia Copik (Burnett School of Biomedical Sciences, University of Central Florida, USA). On day 17, an additional NK enrichment step was performed using magnetic beads-based negative selection (Miltenyi Biotec, Gmbh) and the NK ells were subsequently cultured in RPMI1640 medium with the glucose indicated in the figure at $37^{\circ} \mathrm{C}$ in humidified air containing $5 \% \mathrm{CO}_{2}$ with $21 \% \mathrm{O}_{2}$ (Sanyo MCO-20AIC, Sanyo Electric Co, Japan).

\section{Glucose measurement}

Leftover fresh bone marrow samples were harvested from MM patients with active disease or from healthy donors. When feasible, samples were measured directly as a whole bone marrow harvest. Otherwise, samples were centrifuged with speed $1170 \mathrm{~g}$ for 15 minutes at $4^{\circ}$ Celsius, followed by harvesting of the "plasma" fraction which was stored in $-20^{\circ}$ Celsius before the glucose measurement using YSI biochemical analyzer (Salm en Kipp, BV). Under the Dutch law on Research Involving Human Subject, leftover materials from a patient can be used for research and are waived from individual patient's consent. 


\section{Labeling of K562 cells}

One day prior to the cytotoxicity assay, $2 \times 10^{6}$ cells $/ \mathrm{ml} \mathrm{K} 562$ cells were labeled with $3 \mu \mathrm{l}$ CM-Dil cell labeling dye (Thermo Fisher) in PBS according to the manufacturer's instruction. After adding CM-Dil to the cell suspension, cells were incubated for 5 minutes in the incubator at $37^{\circ} \mathrm{C}$ in humidified air containing $5 \% \mathrm{CO}_{2}$ with $21 \% \mathrm{O}_{2}$ (Sanyo MCO20AIC, Sanyo Electric Co, Japan) followed by 15 minutes incubation at $4^{\circ}$ Celsius in the dark. After the last incubation, cells were washed 2 times with PBS and centrifugation (780 $\mathrm{g}$ for 5 minutes at room temperature). Cells were then resuspended in IMDM medium supplemented with 10\% FCS and 1\% Penicillin/Streptomycin and cultured in the incubator at $37^{\circ} \mathrm{C}$ in humidified air containing $5 \% \mathrm{CO}_{2}$ with $21 \% \mathrm{O}_{2}$ (Sanyo MCO20AIC, Sanyo Electric Co, Japan).

\section{Cytotoxicity assay}

For the cytotoxicity assays in Figure 2 and 3, NK cells were cultured in RPMI1640 medium supplemented with 10\% FCS and 1\% Penicillin/Streptamycin containing 500, 1000, 2000, 4000 , or $8000 \mathrm{mg} / \mathrm{L}$ glucose overnight in the presence of $1000 \mathrm{U} \mathrm{IL}-2 / \mathrm{mL}$. For cytotoxicity assays in Figure 4 and 5, on day 17 the purified expanded NK cells were cultured for 4 days in cultured in RPMI1640 medium supplemented with 10\% FCS and 1\% Penicillin/ Streptamycin containing 500, 2000, or 4000 mg/L glucose. On the day of assay, NK cells and Dil-labeled K562 cells were washed and plated in a 96-wells plate with Dil-labeled K562 cells in 1:1 effector:target (E:T) ratio for 4 hours in the presence of 500, 1000, 2000 or $4000 \mathrm{mg} / \mathrm{L}$ glucose (Figure 2 and 3) or 500, 2000, 4000, or $8000 \mathrm{mg} / \mathrm{L}$ glucose (Figure 4 and 5 ). After 4 hours, the assay was stopped by putting the plate on ice and dead cells were stained and percentages of dead cells were determined flow cytometry.

\section{Staining for dead cells and Flow cytometry}

After a 4-hour cytotoxicity assay, cells were washed with PBS (Gibco) and stained first for dead cells using Live/Dead ${ }^{\circledR}$ Fixable Aqua Dead Cell Stain Kit (Molecular Probes ${ }^{\mathrm{TM}}$, USA) for 30 minutes on ice in the dark. Cells were further washed with PBS buffer (PBS, 1\% FCS) and fixed with $1 \%$ paraformaldehyde in PBS solution. All flow cytometric analyses were performed with BD FACS Canto II. Data were analyzed with FlowJo 10.1r5 64 bit software.

\section{Statistical analysis}

All statistical analysis was performed with GraphPad Prism V software (Graphpad Software Inc, San Diego, CA, USA) using non-parametric t-test with repeated measure (Wilcoxon signed rank test) (Figure 1, 2A, and 3A) or 2-way ANOVA (Figure 2B, 3B, 4 and 5). ${ }^{*}$ indicates a $p$-value of $<0.05,{ }^{* *}$ indicates a $p$-value of $<0.01,{ }^{* * *}$ indicates a $p$-value of $<0.001$ 


\section{RESULTS}

\section{MM patients have a lower glucose concentration in the bone marrow than healthy donors}

To get an indication of glucose levels in the bone marrow, we first investigated glucose concentrations in the bone marrow of MM patients and healthy donors. Leftover bone marrow samples from MM patients or healthy donors for a bone marrow transplantation were collected and glucose levels were measured. This showed that the average glucose concentration in the bone marrow of MM patients was significantly lower than in healthy donors $(p<0.001)$. Glucose concentrations ranged between 479 to $1231 \mathrm{mg} / \mathrm{L}$ (mean $=731.8 \mathrm{mg} / \mathrm{L}, \mathrm{SD}=247.6$ ) for MM patients and between $2297-4196 \mathrm{mg} / \mathrm{L}$ (mean = $3337 \mathrm{mg} / \mathrm{L}, \mathrm{SD}=661.5$ ) for healthy donors (Figure 1). Moreover, for the healthy donors, the average glucose level in the bone marrow was higher than the average normal glucose level in the peripheral blood ( $820-1100 \mathrm{mg} / \mathrm{L}$ fasting or up to $1400 \mathrm{mg} / \mathrm{L}$ random).

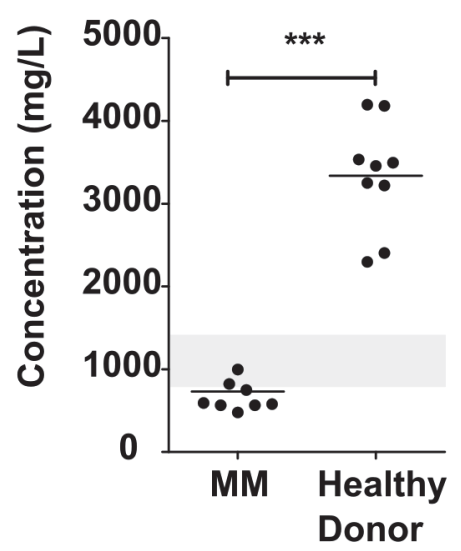

Figure 1. Glucose concentration in the bone marrow of MM patients compared with healthy donors. Bone marrow samples from newly diagnosed MM patients or healthy donors were collected and glucose concentrations were determined using biochemical analyzer (YSI). Grey bar indicates the reference value range for normal fasting blood glucose $(4.4-6.1 \mathrm{mmol} / \mathrm{L}$ or $820-1100 \mathrm{mg} / \mathrm{L}$ up to $1400 \mathrm{mg} / \mathrm{L}$ postprandial). $\mathrm{n}=9$ different subjects for both $\mathrm{MM}$ patients and healthy donors. ${ }^{* * *}=$ $\mathrm{p}<0.001$ 


\section{Low glucose concentrations present during killing did not affect NK cell killing capacity while high glucose is detrimental}

Glucose has been described to be an important fuel for both T cell and NK cell effector functions. Therefore, we tested the effect of low levels of glucose, as observed in MM, and the more physiological levels of glucose, as in the healthy donors, on the cytotoxic capacity of NK cells. For this, we used NK cells isolated from buffy coats that were overnight activated with $1000 \mathrm{U} \mathrm{IL-2/mL}$ in a 4-hour cytotoxicity assay against $\mathrm{K} 562$ cells at 500, 1000, 2000, $4000 \mathrm{mg} / \mathrm{L}$ glucose. The presence of a low concentration of glucose ( $500 \mathrm{mg} / \mathrm{L}$, representing the MM bone marrow) during the 4 h cytotoxicity assay did not negatively affect the killing capacity of IL-2 activated buffy coat-derived NK cells or IL-2 activated PM21-expanded NK cells as compared to conditions with $1000 \mathrm{mg} / \mathrm{L}$ which are more representative for the glucose concertation in the blood of healthy donors (Figure 2A). However, compared to $500 \mathrm{mg} / \mathrm{L}$, higher glucose concentrations $2000 \mathrm{mg} / \mathrm{L}$ (comparable to standard culture conditions, $\mathrm{p}<0.01$ ) or $4000 \mathrm{mg} / \mathrm{L}$, (comparable to normal bone marrow concentrations, $\mathrm{p}<0.01$ ) reduced the cytotoxicity of IL-2 activated NK cells against K562 cells (Figure $2 \mathrm{~A}$ ).

A

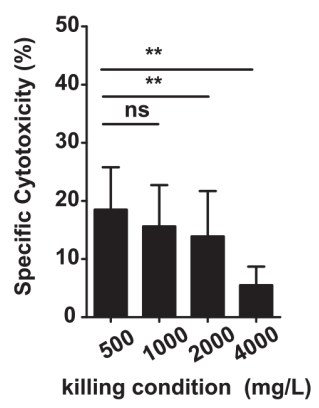

B

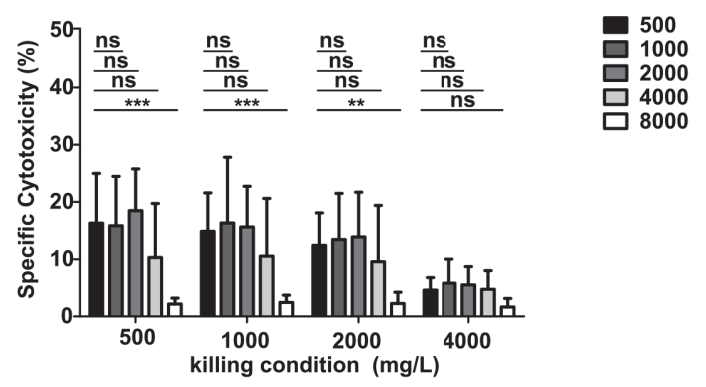

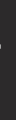
. 
As NK cells could be exposed for a longer period to low glucose levels while traveling through the bone marrow, we further investigated whether low glucose concentrations did have a negative impact on NK cell cytotoxicity when NK cells were exposed to low glucose concentrations during a period of overnight activation. We observed that the exposure to $500 \mathrm{mg} / \mathrm{L}$ up to $4000 \mathrm{mg} / \mathrm{L}$ glucose during overnight activation with IL-2 did not result in a lower cytotoxicity against $\mathrm{K} 562$ cells regardless the glucose concentrations present during killing process (Figure 2B). Significant reductions of NK cell cytotoxicity were detected when $500 \mathrm{mg} / \mathrm{L}$ ( $\mathrm{p}<0.001), 1000 \mathrm{mg} / \mathrm{L}$ ( $p<0.001$ ), and $2000 \mathrm{mg} / \mathrm{L}$ glucose were present during killing process for NK cells cultured in the presence of an extremely high glucose concentration of $8000 \mathrm{mg} / \mathrm{L}$ during an overnight activation (Figure $2 \mathrm{~B}$ ). This reduction in NK cell cytotoxicity was unlikely due to high osmolarity caused by the high glucose levels as we did not see a reduction in NK cell cytotoxicity when NK cells were cultured in the presence of $1000 \mathrm{mg} / \mathrm{L}$ glucose and $7000 \mathrm{mg} / \mathrm{L}$ Mannitol (Supplementary Figure 1).

In summary, these results showed that the presence of low glucose levels during the process of killing or during overnight activation with IL-2 did not reduce NK cell tumorkilling capacity. Quite the opposite, the presence of higher levels glucose concentration (4000 or $8000 \mathrm{mg} / \mathrm{L}$ ) during killing or overnight activation could potentially diminish NK cell cytotoxic capacity and this was unlikely due to a high osmolarity.

\section{A lower glucose concentration during short-term activation conferred a better survival for NK cells during the kill assay}

NK cells typically die after having killed a certain number of cells. We therefore investigated the effect of glucose concentration on NK cell viability during a kill assay. When we analyzed the percentage of dead NK cells in the same kill assay as in Figure 2, we did not observe differences between the percentages of dead NK cells among the different glucose conditions. This suggested that glucose concentrations present during the process of killing did not influence NK cell viability (Figure 3A).

However, when we analyzed the percentage of dead NK cells in the kill assay with NK cells that were cultured overnight with the different glucose concentrations, NK cells cultured overnight in $500 \mathrm{mg} / \mathrm{L}$ glucose had a lower percentage of dead NK cells in the kill assay compared to NK cells cultured in the presence of $4000(p<0.01)$ or $8000 \mathrm{mg} / \mathrm{L}$ glucose $(p$ $<0.001$ ) (Figure 3B). This phenomenon was observed regardless glucose concentrations present during the killing assay. There were no significant differences in the percentage of dead cells between NK cells cultured overnight in $500 \mathrm{mg} / \mathrm{L}$ with $1000 \mathrm{mg} / \mathrm{L}$ or 2000 $\mathrm{mg} / \mathrm{L}$ glucose.

Altogether these viability data showed that the presence of a low glucose concentration either during kill assay or during in vitro overnight activation did not affect NK cells viability negatively. Additionally, low glucose concentration seemed to be advantageous for NK cell survival during the kill assay. 
A

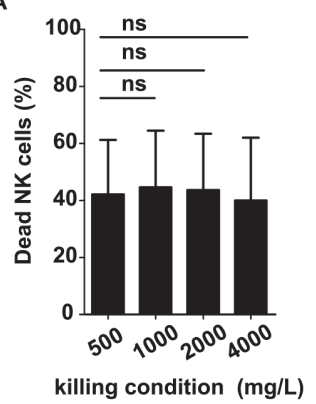

B

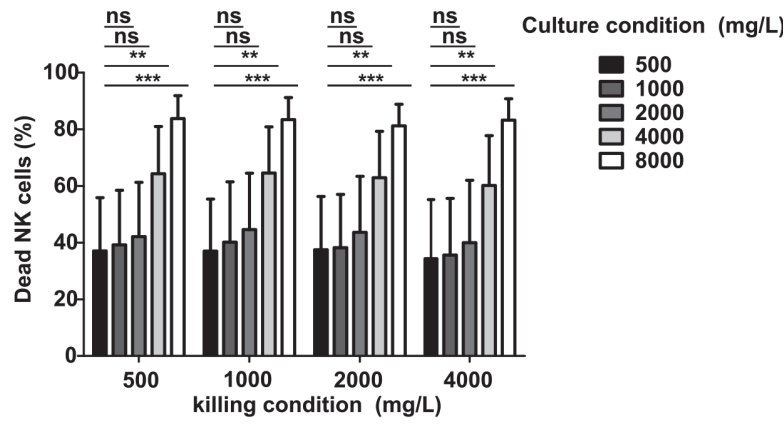

Figure 3. Glucose concentration during killing does not affect NK cell viability in a cytotoxicity assay and NK cells cultured short term lower glucose concentration are less sensitive to dying during killing. (A) Overnight IL-2 activated NK cells cultured in $2000 \mathrm{mg} / \mathrm{L}$ glucose were cocultured with Dil-labeled K562 cells in 1:1 E:T ratio in a 4-hour cytotoxicity assay in different glucose concentrations. (B) IL-2 activated NK cells cultured in different glucose concentrations were cocultured with Dil-labeled K562 cells in 1:1 E:T ratio in a 4-hour cytotoxicity assay in different glucose concentrations. Dead cells were stained with Live/Dead Marker. Shown in the figure is the percentage of dead NK cells during the cytotoxicity assay.

$\mathrm{n}=5$ donors in 3 independent experiments, error bars indicate SD. ${ }^{* *}=p<0.01,{ }^{* * *}=p<0.001$ 


\section{NK cells cultured at lower levels of glucose are better capable of killing target cells and less prone to die in the process of killing}

Clinical application of NK cells requires large numbers of NK cells requiring ex vivo NK cell expansion. Many of the current NK cell expansion protocols use culture media with glucose concentrations of $2000 \mathrm{mg} / \mathrm{L}$ to $4000 \mathrm{mg} / \mathrm{L}$ glucose. Since our data with the shortterm cultures suggested that lower glucose levels might be beneficial, we investigated whether lower glucose levels during ex vivo expansion could improve the killing capacity or the viability of expanded NK cells. For this purpose, CD3-depleted PBMCs from buffy coats were ex vivo-expanded using 2 clinically applicable protocols; a) in the presence of $1000 \mathrm{U}$ IL-2/mL or b) $100 \mathrm{U}$ IL-2/mL in combination with PM21 particles. After 17 days, the obtained NK cells were cultured in 500, 2000, or $4000 \mathrm{mg} / \mathrm{L}$ glucose for an extra 4 days followed by a 4-hour cytotoxicity assay with K562 cells. This showed that NK cells that were cultured at $500 \mathrm{mg} / \mathrm{L}$ killed a higher percentage of target cells than NK cells that were cultured at $2000 \mathrm{mg} / \mathrm{L}$ or at $4000 \mathrm{mg} / \mathrm{L}$ (Figure 4). This was true for the conditions where the actual cytotoxicity assay was performed at $500 \mathrm{mg} / \mathrm{L}, 2000 \mathrm{mg} / \mathrm{L}$ or at 4000 $\mathrm{mg} / \mathrm{L}$. The only exception was the condition where the cytotoxicity assay was performed at $8000 \mathrm{mg} / \mathrm{L}$ and there we did not observe a benefit of four days culture at $500 \mathrm{mg} / \mathrm{L}$. Moreover, at the $8000 \mathrm{mg} / \mathrm{L}$ killing condition, cytotoxicity for all NK products was lower than for the conditions with the cytotoxicity assay at $500 \mathrm{mg} / \mathrm{L}, 2000 \mathrm{mg} / \mathrm{L}$ or at 4000 $\mathrm{mg} / \mathrm{L}$ and the 4 day culture at $500 \mathrm{mg} / \mathrm{L}$ did not improve killing function of the NK cells. In this study we showed earlier that a high glucose concentration present in a shortterm activation culture could affect NK cell viability during the kill assay, we therefore questioned whether this was also true for expanded NK cells. In the same assay as performed in Figure 5, we determined the number of dead NK cells present after a 4-hour cytotoxicity assay. We observed a remarkably high percentage of dead NK cells when NK cells were cultured in 2000 or 4000 mg/L irrespective to the glucose concentration present during the assay. The percentage of dead NK cells at the end of the cytotoxicity assay was much lower for the NK product where NK cells were cultured for four days at $500 \mathrm{mg} / \mathrm{L}$ vs the products cultured at $2000 \mathrm{mg} / \mathrm{L}$ or $4000 \mathrm{mg} / \mathrm{L}$ (Figure 5). This was irrespective of the level of glucose during the cytotoxicity assay. In addition, there was no difference in the percentage of dead NK cells between conditions with the cytotoxicity assay performed at $500 \mathrm{mg} / \mathrm{L}, 2000 \mathrm{mg} / \mathrm{L}$ or $4000 \mathrm{mg} / \mathrm{L}$. However, the percentage of dead NK cells was slightly higher when the cytotoxicity assay was performed at $8000 \mathrm{mg} / \mathrm{L}$. These results suggest that NK cells cultured for 4 days in the presence of a low glucose concentration are better killers and less prone to die in the process of killing as compared to NK cells cultured at higher levels of glucose. These data showed that lower glucose concentrations during a long term culture could be essential for NK cell's survival in a kill assay. 


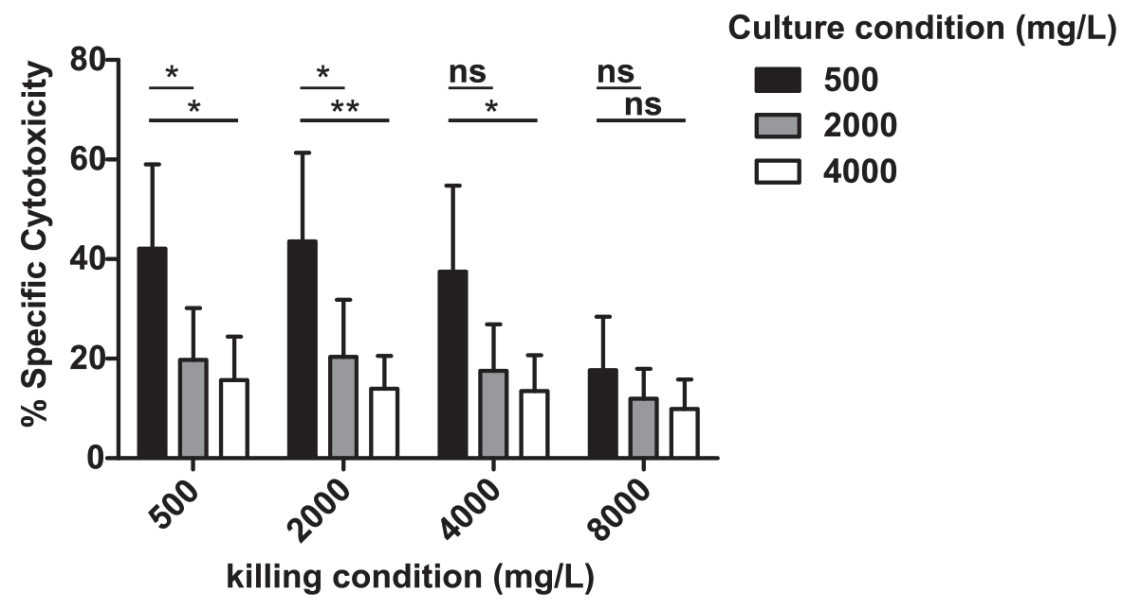

Figure 4. Lower glucose concentration during a longer culture period could improve NK cell cytotoxicity. After 17 days of culture, expanded NK cells were cultured in different glucose concentrations for 4 days. On day 21, NK cells were co-cultured with Dil-labeled K562 cells in 1:1 E:T ratio in a 4-hour cytotoxicity in different glucose concentrations. Dead cells were stained with Live/Dead Marker. Percentage of tumor cells killed by NK cells are denoted as percentage specific cytotoxicity. $\mathrm{n}=4$ samples, $^{*}=\mathrm{p}<0.05,{ }^{* *}=\mathrm{p}<0.01$

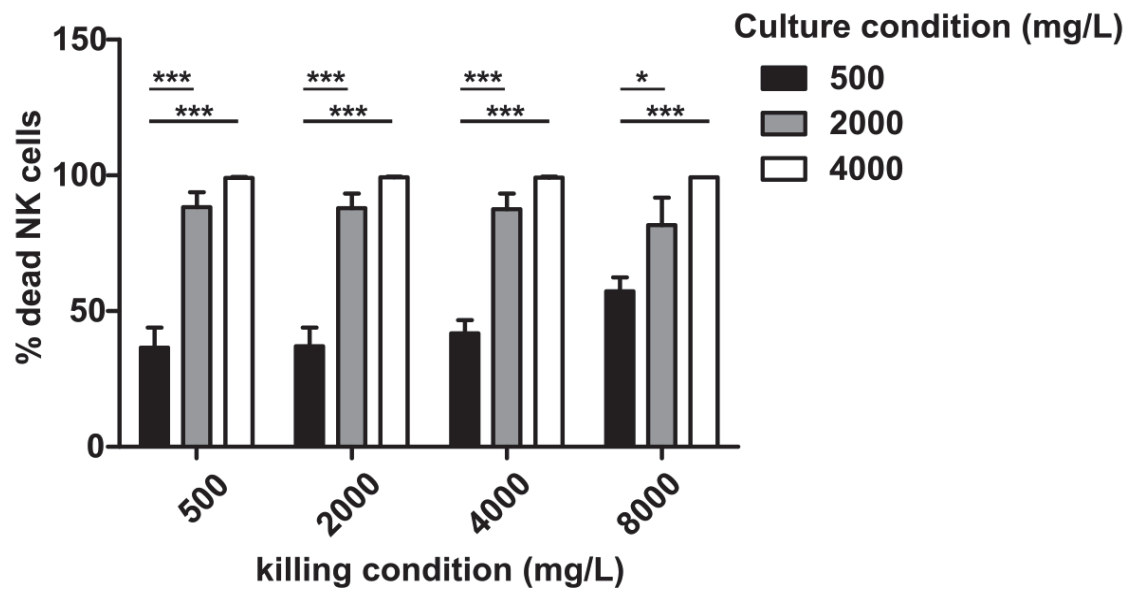

Figure 5. NK cells cultured in lower glucose concentration during a longer culture period are less sensitive to dying during killing. Expanded NK cells were co-cultured with Dil-labeled K562 cells in 1:1 E:T ratio in a 4-hour cytotoxicity assay in different glucose concentrations. Dead cells were stained with Live/Dead Marker. Shown in the figure is the percentage of dead NK cells during the cytotoxicity assay.

$\mathrm{n}=4$ samples, ${ }^{*}=\mathrm{p}<0.05,^{* * *}=\mathrm{p}<0.001$ 


\section{DISCUSSION}

Cancer cells have been demonstrated to be able to reprogram their cellular metabolism [25] and recent studies have shown that this metabolic reprogramming could have a negative impact on the antitumor response of immune cells due to metabolic competition [26]. Moreover, a limited glucose supply and the increase in lactate concentration as a by-product of aerobic glycolysis have been shown to render T cells exhausted or hyporesponsive [26]. While the metabolic requirement for T cells have been heavily investigated, NK cell metabolism is still largely unexplored and this is especially true for the effect of metabolism on the cytotoxic capability of highly activated human NK cells. In the current study, we therefore aimed to gain more insight on the effect of tumor metabolic microenvironment on highly activated NK cells antitumor response.

First, we showed that glucose concentrations in the bone marrow of MM patients with active disease were lower than the glucose concentrations in healthy donors. These MM glucose concentrations, however, are within the range of the normal blood glucose and are still higher than the concentrations found in the solid tumors [16]. Furthermore, we observed that short-term exposure to these lower glucose concentrations did not have a detrimental effect on the killing capacity or viability of NK cells. Although we did not investigate the underlying mechanism in detail, an explanation could be that our NK cells were peripheral blood-derived NK cells, meaning that a very large proportion of the NK cells in the assays were CD56 dim NK cells. Different human NK cell subsets have been shown to possess different metabolic requirements. CD56 bright NK cells, which are the main cytokine producers, appear to be metabolically more active than CD56 dim NK cells upon cytokine stimulation [23]. Therefore, CD56 bright NK cells are more likely to suffer more from the restricted glucose in the environment. In addition, during activation, NK cells are able to perform metabolic reprogramming, with a preference for glycolysis towards OxPhos [27]. Since we used high dose IL-2 activated NK cells, these cells could have become more or less independent on the availability of glucose. Unlike T cells that are more dependent on glucose's availability to become activated, NK cells might be the better tumor-cells killer in the area where low glucose concentrations are located.

Interestingly, a high glucose in our assays concentration did result in a reduction in NK-cell cytotoxicity as well as an increase in the percentage of dead NK cells during the kill assay. This observation was highly unlikely due to high molarity since we did not observe the effect with mannitol. Furthermore, it was in line with a previous study on unactivated human NK cells showing that short-term exposure of NK cells to 8000 
$\mathrm{mg} / \mathrm{L}$ glucose led to an increase in intracellular calcium ion concentration, which is vital to NK cell cytotoxicity, and resulted in inhibition of NK cell cytotoxicity [28].

Many clinical protocols, aiming at NK cell infusion as a mean for cancer immunotherapy, will require an infusion of extremely high numbers of NK cells which necessitates ex vivo NK cell expansion. We therefore anticipated that this expansion period could provide an opportunity to either prime NK cells for the metabolic conditions in the tumor or to enhance their function or persistence by interfering with their metabolic programming. We indeed observed that a relatively low glucose concentration, 500 $\mathrm{mg} / \mathrm{L}$ vs 2000 or $4000 \mathrm{mg} / \mathrm{L}$ as present in most standard culture media, during the last four days of culture resulted in the highest NK cell cytotoxic response against K562 cells as well as the best NK cell viability after the cytotoxicity assay. And similar to our short-term culture data, higher glucose culture concentrations during the last four days of culture resulted in lower percentages of NK-cell specific cytotoxicity against tumor cells. This data also implied that a period of acclimatization to a higher glucose concentration did not result in an improvement of NK cell cytotoxic capacity. Additionally, a period of acclimatization to a lower glucose after a higher glucose concentration during expansion protocol seemed to result in better killer cells. For tumor-infiltrating T cells (TILs), it has been shown that low glucose levels during the ex vivo expansion enhanced TIL persistence upon transfer into tumor-bearing immunodeficient mice and by doing so overall anti-tumor responses [29, 30]. While we are currently investigating the underlying mechanism behind our data, they clearly show the potential of lowering glucose levels, at least during the last days of culture, to achieve a more optimal NK cell product.

In conclusion, our current findings showed that exposure to a lower glucose concentration representing the MM bone marrow either short-term or long-term did not have detrimental effect on NK-cell cytotoxic capacity against tumor cells. Although this is positive news for NK cell-based immunotherapy, future studies should be directed to answer the mechanism why, despite the need of aerobic glycolysis for its effector function, low glucose concentration seemed to be even advantageous for NK cell antitumor capacity. In addition, our study demonstrates that interfering with NK cell metabolism during ex vivo NK cell expansion could be a novel way to yield more potent NK cells that might in the future contribute to improved clinical efficacy. 


\section{REFERENCES}

1. Fang F, Xiao W, Tian Z (2017) NK cell-based immunotherapy for cancer. Semin Immunol 31:3754. doi: 10.1016/j.smim.2017.07.009

2. Liang S, Xu K, Niu L, et al (2017) Comparison of autogeneic and allogeneic natural killer cells immunotherapy on the clinical outcome of recurrent breast cancer. Onco Targets Ther 10:42734281. doi: $10.2147 /$ OTT.S139986

3. Veluchamy JP, Kok N, van der Vliet HJ, et al (2017) The rise of allogeneic Natural killer cells as a platform for cancer immunotherapy: Recent innovations and future developments. Front Immunol. doi: 10.3389/fimmu.2017.00631

4. Bachanova V, Miller JS (2014) NK cells in therapy of cancer. Crit Rev Oncog 19:133-41. doi: 10.1615/CritRevOncog.2014011091

5. Larsen SK, Gao Y, Basse PH (2014) NK cells in the tumor microenvironment. Crit Rev Oncog 19:91-105. doi: 10.1016/j.biotechadv.2011.08.021.Secreted

6. Chiu J, Ernst DM, Keating A (2018) Acquired Natural Killer Cell Dysfunction in the Tumor Microenvironment of Classic Hodgkin Lymphoma. Front Immunol 9:267. doi: 10.3389/ fimmu.2018.00267

7. Mocellin S, Wang E, Marincola FM (2001) Cytokines and immune response in the tumor microenvironment. J Immunother 24:392-407. doi: 10.1097/00002371-200109000-00002

8. Jewett $\mathrm{A}$, Tseng H-C (2011) Tumor induced inactivation of natural killer cell cytotoxic function; implication in growth, expansion and differentiation of cancer stem cells. J Cancer 2:443-57. doi: $10.7150 /$ jca.2.443

9. Sarkar S, Germeraad WT V, Rouschop KMA, et al (2013) Hypoxia induced impairment of NK cell cytotoxicity against multiple myeloma can be overcome by IL-2 activation of the NK cells. PLoS One 8:e64835. doi: 10.1371/journal.pone.0064835

10. Balsamo M, Manzini C, Pietra G, et al (2013) Hypoxia downregulates the expression of activating receptors involved in NK-cell-mediated target cell killing without affecting ADCC. Eur J Immunol 43:2756-2764. doi: 10.1002/eji.201343448

11. Baginska J, Viry E, Paggetti J, et al (2013) The Critical Role of the Tumor Microenvironment in Shaping Natural Killer Cell-Mediated Anti-Tumor Immunity. Front Immunol. doi: 10.3389/ fimmu.2013.00490

12. Pietra G, Manzini C, Rivara S, et al (2012) Melanoma cells inhibit natural killer cell function by modulating the expression of activating receptors and cytolytic activity. Cancer Res 72:14071415. doi: 10.1158/0008-5472.CAN-11-2544

13. Vitale M, Cantoni C, Pietra G, et al (2014) Effect of tumor cells and tumor microenvironment on NK-cell function. Eur J Immunol 44:1582-1592. doi: 10.1002/eji.201344272

14. Kroemer G, Pouyssegur J (2008) Tumor Cell Metabolism: Cancer's Achilles' Heel. Cancer Cell 13:472-482. doi: 10.1016/j.ccr.2008.05.005

15. DeBerardinis RJ, Lum JJ, Hatzivassiliou G, Thompson CB (2008) The Biology of Cancer: Metabolic Reprogramming Fuels Cell Growth and Proliferation. Cell Metab 7:11-20. doi: 10.1016/j. cmet.2007.10.002

16. Hu X, Chao M, Wu H (2017) Central role of lactate and proton in cancer cell resistance to glucose deprivation and its clinical translation. Signal Transduct Target Ther 2:16047. doi: 10.1038/ sigtrans.2016.47

17. Pearce EL, Pearce EJ (2013) Metabolic pathways in immune cell activation and quiescence. Immunity 38:633-643. doi: 10.1016/j.immuni.2013.04.005

18. Sukumar M, Roychoudhuri R, Restifo NP (2015) Nutrient Competition: A New Axis of Tumor Immunosuppression. Cell 162:1206-1208. doi: 10.1016/j.cell.2015.08.064 
19. Chang CH, Qiu J, O'Sullivan D, et al (2015) Metabolic Competition in the Tumor Microenvironment Is a Driver of Cancer Progression. Cell 162:1229-1241. doi: 10.1016/j.cell.2015.08.016

20. Marçais A, Cherfils-Vicini J, Viant C, et al (2014) The metabolic checkpoint kinase mTOR is essential for IL-15 signaling during the development and activation of NK cells. Nat Immunol 15:749-757. doi: 10.1038/ni.2936

21. Keppel MP, Saucier N, Mah AY, et al (2015) Activation-specific metabolic requirements for NK Cell IFN- $\gamma$ production. J Immunol 194:1954-62. doi: 10.4049/jimmunol.1402099

22. Donnelly RP, Loftus RM, Keating SE, et al (2014) mTORC1-Dependent Metabolic Reprogramming Is a Prerequisite for NK Cell Effector Function. J Immunol 193:4477-4484. doi: 10.4049/ jimmunol.1401558

23. Keating SE, Zaiatz-BittencourtV, Loftus RM, et al (2016) Metabolic Reprogramming Supports IFN- $\gamma$ Production by CD56 bright NK Cells. J Immunol 196:2552-2560. doi: 10.4049/jimmunol.1501783

24. Oyer JL, Igarashi RY, Kulikowski AR, et al (2015) Generation of highly cytotoxic natural killer cells for treatment of acute myelogenous leukemia using a feeder-free, particle-based approach. Biol Blood Marrow Transplant 21:632-639. doi: 10.1016/j.bbmt.2014.12.037

25. Hanahan D, Weinberg RA (2011) Hallmarks of cancer: the next generation. Cell 144:646-74. doi: 10.1016/j.cell.2011.02.013

26. Buck MD, Sowell RT, Kaech SM, Pearce EL (2017) Metabolic Instruction of Immunity. Cell 169:570-586. doi: 10.1016/j.cell.2017.04.004

27. Gardiner CM, Finlay DK (2017) What fuels natural killers? Metabolism and NK cell responses. Front Immunol. doi: 10.3389/fimmu.2017.00367

28. Whalen MM (1997) Inhibition of Human Natural Killer Cell Function in vitro by Glucose Concentrations Seen in Poorly Controlled Diabetes. Cell Physiol Biochem 7:53-60. doi: $10.1159 / 000154852$

29. Sukumar M, Liu J, Ji Y, et al (2013) Inhibiting glycolytic metabolism enhances CD8+T cell memory and antitumor function. J Clin Invest 123:4479-4488. doi: 10.1172/JCI69589

30. Chang C, Qiu J, O'Sullivan D, et al (2015) Metabolic Competition in the Tumor Microenvironment Is a Driver of Cancer Progression. Cell 162:1229-1241. doi: 10.1016/j.cell.2015.08.016 


\section{SUPPLEMENTARY FIGURE}

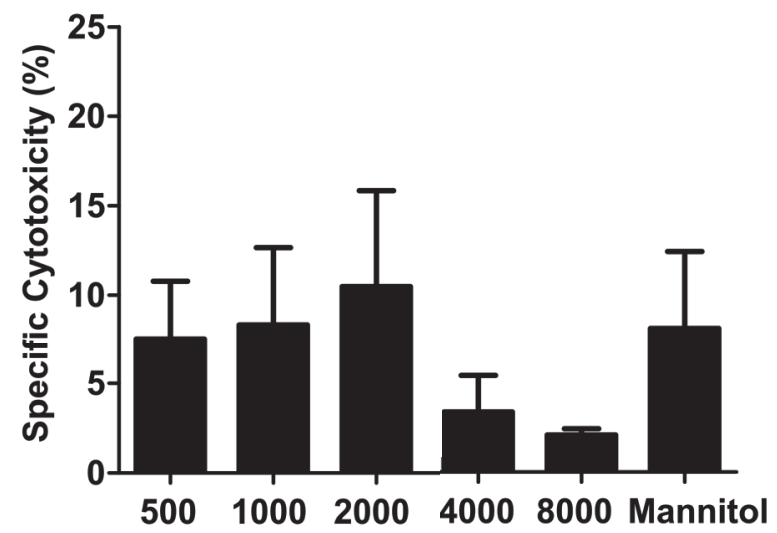

Supplementary Figure 1. High osmolarity is not the cause of a reduced cytotoxicity capacity of NK cells due to high glucose concentration during culture. NK cells stimulated with $1000 \mathrm{U} / \mathrm{ml} \mathrm{IL-2}$ were cultured overnight in the presence of different glucose concentrations or high concentration of Mannitol equivalent to $8000 \mathrm{mg} / \mathrm{L}$ glucose. The following day, NK cells were co-cultured with Dillabeled K562 cells in 1:1 E:T ratio in a 4-hour cytotoxicity assay in different glucose concentrations as indicated. Dead cells were stained with Live/Dead Marker. Percentage of tumor cells killed by NK cells are denoted as percentage specific cytotoxicity. $\mathrm{n}=2$ independent experiments. 
Less is more: a low glucose concentration during short and long term cultures is correlated with a better antitumor response and viability of activated NK cells 
\begin{tabular}{l|l}
186 & Chapter 8
\end{tabular} 


\section{General Discussion}




\section{INTRODUCTION}

Multiple myeloma (MM) is a malignancy of plasma cells, which normally produce antibody. Although it is relatively rare, it is the second most commonly diagnosed hematological malignancy [1]. Around 1200 people every year are diagnosed with MM in the Netherlands (Netherlands Cancer Registry) and the worldwide incidence rate is around $0.8 \%$ per year (GLOBOCAN data). The current standard treatment for MM depends on a patient's age. Fit patients younger than 70 years old receive induction therapy followed by a high dose chemotherapy and an autologous stem cell transplantation rescue while unfit patients or patients older than 70 years old receive a different treatment schedule without high dose chemotherapy because of its toxicity [2]. Despite the improvement of progression-free survival in the past few years due to the discovery of various new drugs besides classical chemotherapy such as immunomodulatory drugs (lenalidomide; pomalidomide) proteasome inhibitors (i.e bortezomib, ixazombi) antibodies (daratumumab, elotuzumab) or HiDac inhibitors (panibinostat), MM is still incurable. Nearly all patients will have a relapse after achieving several remission with different treatment strategies [3]. This relapse is likely because MM cells often develop resistance to subsequent treatments by acquiring mutations in targeted pathways [4].

Cellular-immunotherapy could be a novel therapeutic option for MM patients and natural killer (NK) cells might be excellent candidates to target. Several reasons for this notion include:

1. NK cells mediate strong antitumor responses both in vitro and in vivo without the need of priming with (different) tumor-associated/specific-antigens (reviewed in [5]).

2. Donor NK cells do not trigger graft versus host disease (GvHD), even in a human leukocyte antigen (HLA) mismatched setting [5-8]

3. NK cells can be purified and expanded ex vivo in a clinical grade also in a fully automated process [9-12]

4. NK cells can be genetically engineered to improve its efficacy and/or specificity against tumor cells [13-15]

In chapter 6, we put forward two platforms to exploit the anti-MM potential of alloreactive NK cells namely though haploidentical stem cell transplantation (haploSCT) or by infusion of high numbers of alloreactive NK cells. Haplo-SCT is a type of alloSCT where donor and patient are matched for only one of the two HLA haplotypes. Despite its potential cure, the use of allogeneic stem cell transplantation to treat MM 
patients as an alternative to the autologous transplantation, is still limited, due to treatment-related mortality, disease progression, difficulties in inclusion criteria as well as finding suitable donors [16-20]. Nonetheless, the breakthrough paper by Ruggeri et al in 2002 opened up a new treatment opportunity for cancer patients and possibly for MM patients as well. The study found, in patients with acute myeloid myeloma, that alloreactive NK cells in haploidentical stem cell transplantation elicit a graft versus leukemia (GvL) effect without causing a GvHD [21]. In a mouse model of breast cancer, our group has previously demonstrated that indeed NK cells played a crucial role in the tumor elimination in the mice receiving haploidentical transplantation [22]. More recently, haplo-SCT became a realistic treatment option which was mainly due to the implementation of novel strategies to control GvHD disease and since then the number of haplo-SCT is vastly increasing [23, 24]. Our center has started the first phase I/II multicenter study in MM were haplo-SCT where donors were selected on the basis of having a killer immunoglobulin-like (KIR)-ligand mismatch. Although it is too early to draw any real conclusions based on that study, it did already illustrate the feasibility of the approach and underlined that haplo-SCT could be an attractive novel platform for NK cell therapy: the Phase I study has been completed and toxicity is limited and patients are included for the phase II study.

Infusion of high numbers of ex vivo-expanded NK cells is an alternative way of using the antitumor potential of NK cell in MM. The generation, efficacy, and safety of ex vivo-expanded NK cells are currently being heavily investigated by different research groups. Several clinical studies have demonstrated that the source of ex vivoexpanded NK cells could be an NK-cell line such as NK-92 cell [25], umbilical cord blood stem cells [26-28], or peripheral blood/apheresis/buffy coats from healthy donors [29]. To date, there is no standard protocol for ex vivo NK cell expansion. NK cells expanded from the peripheral blood mononuclear cells (PBMCs),either with or without CD3+ depletion, have been cultured with [30-34] or without feeder cells [3539]. As recently reviewed by Koehl et al, although infusions with ex vivo-expanded NK cells were generally well-tolerated and safe, the clinical efficacy of ex vivo-expanded NK cells seems to be limited [40].

Observing the cancer-curing potential of NK cells and their relatively good safety profile, our group aims to develop NK cell-based therapy to treat cancer patients. Although we focused our investigations mainly on MM in this thesis, the general approaches/concepts put forward in the thesis could possibly be applied in the development of NK cell-based therapy for different types of cancers as well. As in detail described in chapter $\mathbf{1}$ and chapter $\mathbf{6}$ of this thesis, the NK cell response is dictated by the sum of activating and inhibitory signals it receives. Therefore, to 
create NK cells that can mediate potent effector functions in patients, in this thesis we aimed to identify limiting factors and boosting strategies that we can apply to maximize NK cell activation and reduce inhibition in the tumor microenvironment (TME) (Figure 6.1). In this general discussion, we will integrate the different chapters of the thesis by discussing the main factors that could limit NK cell therapy, followed by a discussion on current and future strategies that may help to overcome these limitations.

\section{Limiting Factors}

\section{Boosting Strategies}

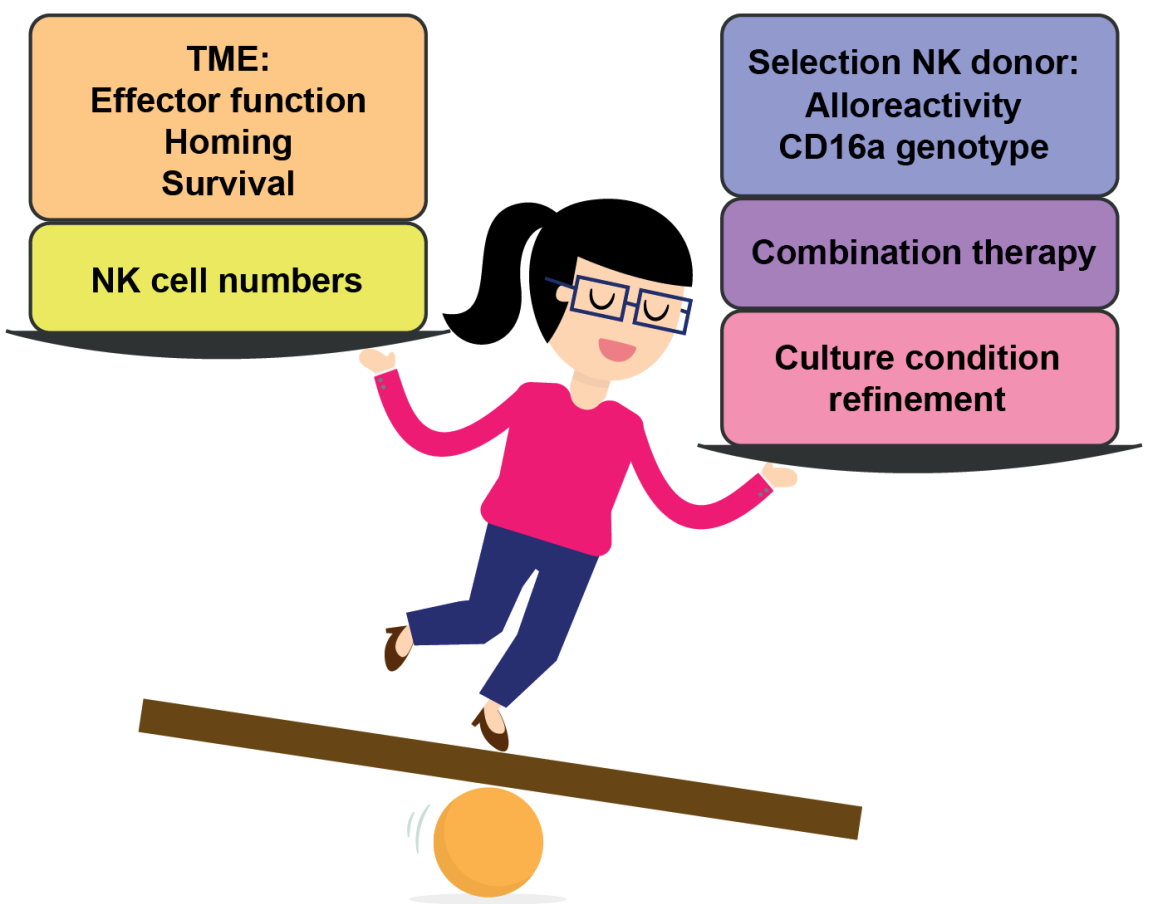

Figure 8.1. Strategies to enhance the efficacy of NK cell-based therapy. Limiting factors described in this thesis include the number of NK cells and tumor microenvironment (TME) which can affect NK cells homing and survival and effector function. Boosting strategies proposed in this thesis include the selection of NK cell donor, combination therapy (i.e. with a monoclonal antibody), and optimizing NK cell culture condition (i.e. optimizing glucose culture condition). 


\section{IDENTIFICATION OF FACTORS THAT CAN LIMIT CLINICAL EFFICACY OF NK CELLS IN MM}

You are a product of your environment. So choose the environment that will best develop you toward your objective. Analyze your life in terms of its environment. Are the things around you helping you toward success - or are they holding you back?

\section{- W. Clement Stone}

The suppressive tumor microenvironment is a major hurdle for NK cellbased therapies

In general, the TME is comprised of cellular and non-cellular compartments. The cellular compartment of the TME is mainly composed of stromal cells, immune cells, blood and lymphatic vessels, adipose cells, neuroendocrine cells, and cancer-associated fibroblasts [41]. Accumulating evidence (as reviewed in [42]) showed that cancerassociated fibroblasts and other immune cells such as tumor-associated macrophages (TAMs), myeloid-derived suppressor cells (MDSCs), regulatory T cells present in the TME play a significant role in controlling and suppressing the NK-cell antitumor response. The mechanisms involved downregulation the activating receptors expressed on NK cells [43-45], reduced NK-cell degranulation [46] and reduced interferon gamma (IFN- $\gamma$ ) production $[47,48]$. The non-cellular compartment of the TME encompasses the extracellular matrix (ECM) and an array of soluble factors secreted in the TME such as growth factors, cytokines, and chemokines [49] as well as (by-) products of metabolism [50]. Soluble factors such as adenosine [51, 52], prostaglandin E2 (PGE2) [53], transforming growth factor beta (TGF-ß) [52], lactate [54, 55], nitric oxide[56] have been demonstrated to compromise NK-cell antitumor response.

Despite evidence that the TME hampers potent antitumor immune cell function, many investigators neglected this fact in the setup of their in vitro experiments. This underrepresentation of the TME in many in vitro experiments may provide one of the possible arguments for discrepancy between the in vitro and in vivo success of NK cell-based therapies. Data from numerous previous in vitro and in vivo mouse studies demonstrated that NK cells exhibited antitumor responses against both cell lines or primary cells of haematological [57-60] and solid cancers [61-66]. However, data from clinical studies showed that not all types of cancer were sensitive to NK cell therapy. Moreover, clinical studies on hematological cancers such as lymphoma 
[67] or leukemia $[68,69]$ demonstrated that some patients could achieve a complete remission or partial response while others did not. Also, the efficacy of NK cellbased therapy on solid tumors was less impressive even though the number of (endogenous) NK cells in solid cancers was positively correlated with the survival of patients, suggesting that NK cells played a vital role in the eradication of tumor cells [70].

MM cells home and reside in the bone marrow (BM) where they attach to the ECM and the stromal cells, and heavily depend on these interactions. The BM milieu is composed of both cellular and non-cellular compartment. The cellular compartment is comprised of hematopoietic cells, endothelial cells, osteoclasts, osteoblasts, and fibroblasts [71]. The non-cellular compartment consists of the ECM and enriched with soluble factors such as growth factors and cytokines crucial for the homeostasis of both hematopoietic stem cells and progenitors. Additionally, the BM is physiologically more hypoxic than many other tissues [72]. A recent study showed that MM cells could adapt to a long exposure of hypoxia by exhibiting stem-cell characters via the activation of TGF-ß/Smad pathway [73]. In patients with MM, previous studies have reported that there was an accumulation of regulatory T cells [74, 75], myeloidderived suppressor cells [76], and macrophages [77] in the BM of MM patients. Additionally, as reviewed by Pittari et al, soluble factors such as TGF- B, IL-10, IL-6, PGE2, Indoleamine 2,3-dioxygenase, and soluble MHC-related ligands (sMICs) are increased in the MM BM [78]. IL-6, produced by BM stromal cells, is an important growth and survival factor for MM and found abundantly in the BM. In addition, this cytokine could induce Stat3 signaling in MM cells, conferring protection from Fasmediated apoptosis [79]. Furthermore, gene analysis of primary MM cells showed that aerobic glycolysis is functional in $M M$ and that $M M$ cell lines produced high amount of lactate as a result of glycolysis[80]. Several of these MM TME factors have been shown to support MM growth and survival and contribute to MM resistance to therapeutic agents as well as to anti-tumor effector functions.

Given that the immune cells and soluble factors present in the MM BM have been previously reported to potentially diminish NK-cell antitumor response [42, 55, 78], we felt the importance to create a more representative in vitro condition in our studies and performed most of our in vitro studies in the presence of TME factors. One important factor in our studies was hypoxia. Our group previously showed that hypoxia could diminish NK-cell anti-MM activity [81]. We also showed that preactivation of NK cells with a high dose of IL-2 could restore the NK-cell cytotoxicity capacity against MM cell lines under hypoxia. Nonetheless, there are multiple factors which can simultaneously present in the TME. In this thesis, we therefore took it one 
step further by investigating the effect of combinations of TME factors. In chapter $\mathbf{2}$ and 5, we proved that although IL-2 activation could restore NK-cell antitumor response under hypoxia, it is not enough to support NK-cell antitumor activity when another suppressive factor(s) are also present. One of these factors was lactate, present in the TME of many tumors as a by-product of tumor-cell metabolism [82] and shown to be harmful for NK-cell antitumor activity [54,55]. We showed that a high concentration of lactate during killing process could blunt IL-2 activated NK-cell antitumor response. The coexistence of hypoxia led to a more severe incapacitation of NK-cell killing. Surely our representation of TME in our studies was a simplification of the real complexity of in vivo situation, but even with the combination of only two suppressive factors, we showed that it is important to realize that TME is a major hurdle for NK-cell antitumor response. Therefore, further identification of suppressive TME factors that could disarm NK cell-based therapy efficacy is pivotal.

In our effort to identify these factors, we followed the other end of the consequence of an altered tumor cell metabolism (chapter 7). While the concentration of lactate builds up, the glucose level in the TME drops as a result of glucose consumption. The lack of glucose availability in the TME could result in metabolic competition between tumor cells and the immune cells. Extensive studies on T cells showed that this competition contributed to CD8+ T cell dysfunction and anergy (reviewed in [83]), as $\mathrm{T}$ cells also rely on glucose to execute their effector function. To date, there is, however, very limited data on the effect of glucose concentration on NK-cell tumorkilling capacity. Additionally, data on the exact glucose concentration in the TME is scarce. Although MM is regarded a slow-growing malignancy, we showed for the first time that in the BM of patients with active disease, the glucose levels were found to be lower than the glucose levels of that of healthy donors (chapter 7). However, to our surprise, unlike high lactate concentrations, lower glucose concentrations present during killing process did not negatively affect IL-2 activated NK-cell killing and our results pointed out that, unlike CD8+ T cells, NK cells did not seem to rely on glucose to kill a tumor cell. On the contrary, NK cells might even prefer a lower glucose concentration and such lower glucose concentrations, either during activation process or (longer) culture period, resulted in a better cytotoxicity against K562 cells. Apparently, a high glucose concentration seemed to predispose NK cells to be more susceptible to death in the culture as well as during kill assay. Studies on diabetic models on other cell types suggested several probable mechanisms such as altered metabolism [84], the formation of hydrogen peroxide [85], free radical generation and oxidative stress in the mitochondria [86]. Since we did not dig into the underlying mechanisms yet, it would be interesting to further dissect the exact mechanism of this susceptibility in the context of TME. 
Besides the direct suppressive effects of the TME on the NK cell antitumor response, other important issues that we need to tackle with regards to the TME are the homing to- and the survival of NK cell in the tumor bed. Several studies on solid tumors showed that the number of NK cells present in the TME was frequently low and that the NK cells were functionally impaired reviewed in [87]. NK cells present in the BM of MM patients displayed characteristics of an exhausted phenotype seen by the decreased expression of activating receptors (such as CD16 [88], 2B4[88, 89], NKp30[89], and NKG2D[89]) and an upregulation of the programmed death-1 receptor (PD-1)[90]. In addition, NK cells present in the TME are often located closer to blood vessels and not directly in contact with the tumor cells. The complexity of the TME, as well as the secretion of cytokines / chemokines / chemoattractants in established solid tumors play a major role in hampering NK cells infiltration to the tumor bed [91]. A previous study demonstrated that the growth of MM cells both in a mouse model of $M M$ as well as in patients caused a perturbed chemokinesprofile in the BM resulting in a defect of NK cell homing to- and retention in the BM as well as reduced degranulation capacity [92]. The observation in the BM of MMbearing mice and in the serum of MM patients showed an upregulation of CXCL9 and CXCL10 (CXCR3 ligands) followed by a downregulation of CXCR3 expression on NK cells and decreased CXCL12. Nonetheless, In a NOD/scid/IL2Rynull mice model for MM, expanded human NK cells have been shown circulating in the peripheral blood, trafficking to the tumor site, persisting in the MM TME, and protected the bone from MM-mediated destruction [93]. In patients with relapsed MM, infusions of high dose ex vivo-expanded NK cells were well tolerated and significant NK cells expansion in vivo was observed [7]. However, due to a limited number of patients recruited in the study, it is difficult to really evaluate the clinical efficacy of the NK cell therapy. Data on expanded NK cell biodistribution in patients, especially in the MM TME, would be valuable to provide insight to the fate of the infused expanded NK cells. In our clinical phase I study we observed that donor NK cells are present in the bone marrow 30 and 60 days after transplants. This clinical model will give us the opportunity to analyze the potency of donor NK cells in MM (unpublished observations). Despite the lack of patient's data on expanded NK cells fate in the BM of MM patients, we can safely speculate that the TME of MM could present a potential challenge for NK cell-based therapy.

Altogether, we presented in this thesis that TME factors are important aspects to take into account in designing an NK cell-based therapy for cancer patients. Therefore, we would like to emphasize 3 points: 1) the identification of relevant TME factors is very crucial for the success of NK cell-based therapy or any other kind of cancer therapy. As each cancer type is unique, TME factors can vary per cancer type. Techniques 
including three-dimensional techniques to identify the tumor microenvironment are available to date $[94,95]$ and may be helpful to identify and prioritize targeting of TME factors. 2) Future in vitro experiments should include TME factors to better represent a more realistic situation and provide a better prediction on the tested (combination) therapy. Ideally, an in vitro testing condition should closely mimic the in vivo environment of a tumor. For this reason, utilizing a more complex in vitro tumor model, especially for solid tumors, for example using a scaffold model, a tumor spheroid model or a hybrid model (by embedding ex vivo tumor sections) would be a better approach for an in vitro testing (reviewed in [96]). An affordable and userfriendly image-based approach for 3D spheroid tumor model has been recently developed to facilitate the monitoring of tumor cell growth and cytotoxicity [97]. 3) In addition to NK-cell activation status, NK-cell numbers, homing capacity, and survival in the tumor bed play an important role in the success of NK-cell antitumor response in vivo.

Therefore, protocol optimizations for ex vivo expansion of NK cells should also focus on the improvement of NK-cell survival in vivo and include the evaluation of expanded NK-cell homing capacity.

\section{POTENTIAL STRATEGIES TO CREATE TUMOR-KILLER NK CELLS WHICH CAN WITHSTAND THE CHALLENGE FROM THE TME}

In most cases, strengths and weaknesses are two sides of the same coin. A strength in one situation is a weakness in another, yet often the person can't switch gears

\section{Minimizing the inhibitory signaling: choosing the right donor and blocking the right receptor}

Given that NK-cell reactivity against a target cell is determined by the integration of both inhibitory and activating signals [98], minimizing inhibitory signal and maximizing activation signal would be a strategy to unleash the NK-cell antitumor response. Such strategies are necessary since components of the TME, both cellular and non-cellular could dampen NK-cell activation and provide strong inhibitory 
signals leading to NK-cell exhaustion or anergy and poor NK-cell effector function [99]. We set out to address several strategies to enhance NK cell function in a suppressive environment starting with minimizing the inhibitory signals. One of the biological advantages of NK cells is that because allogeneic NK cells do not trigger GvHD on both mice models and patients and that NK cells could be isolated, activated and used from allogeneic sources. As this provides extensive possibilities to select and obtain the best and most potent NK-cell donors, we first evaluated the functional relevance of including KIR-ligand mismatching in donor selection criteria for MM.

Tumor cells often downregulate their surface HLA expression to avoid T-cell surveillance, rendering their susceptibility to NK-cell-mediated killing [100]. Nonetheless, primary MM cells from patients showed that this is not always the case as they were frequently found to stably express classical class I HLA molecules [101], conferring their resistance to NK-cell-mediated lysis [102]. The observation that HLA is highly expressed in MM provides a rationale for the selection of KIR-ligand mismatched NK-cell donor for NK cell-based adoptive therapy for MM as an effort to minimize inhibitory signaling and to reduce the activation threshold as much as possible. Our group previously reported that killer-immunoglobulin-like receptor (KIR)-ligand mismatched NK cells were the better effector cells compared to the KIRligand matched NK cells against MM [101]. In this thesis, we followed up on these findings by showing that KIR-ligand mismatched NK cells are even better in the situation where the NK cell receives a lot of extra activating signals via an antibodydependent cell-mediated (ADCC) triggering antibody (chapter 2) as well as primary MM cells isolated from MM patients (chapter 5). The selection of an ideal NK-cell donor should therefore be based on the KIR-ligand mismatched status with the recipients. Furthermore, it is important that the NK cells are fully licensed to allow alloreactivity against the cancer cell [103].

Next, we investigated the feasibility to create a KIR-ligand mismatched NK cells to treat MM patients, we performed a pilot study comparing the genotypic expression of HLA epitopes in healthy subjects and in MM patients showing that $70 \%$ of MM patients miss one or two HLA epitopes and therefore could benefit from KIR-ligand mismatched NK cells (chapter 6). Moreover, 30\% of healthy donors genotypically express all three HLA epitopes meaning that they could be universal alloreactive NKcell donors. Nonetheless, since KIRs and HLA molecules are inherited independently, an additional test to investigate the genotypic and phenotypic expression of KIRs would be required to confirm that the mismatched KIR is expressed by the donor NK cells. Based on the results of our aforementioned study, we propose that selecting a donor NK cell-based on its alloreactivity is a feasible strategy to be applied in 
the clinic. Another important message from this study is that although KIR-ligand mismatched NK cells could potentially benefit the vast majority of patients, $30 \%$ of the patients genotypically express all three HLA-epitopes. Therefore, there is no possible KIR-ligand mismatch.

The lack of possibility to reduce inhibitory signaling by selection of KIR-ligand mismatched donors in 30\% of the MM patients raised another important question that we addressed in this thesis: How can we reduce inhibitory signaling when we already select the best donor based on HLA and KIR genotype? To address this issue, we evaluated the importance of HLA-E and NKG2A interaction in the NK cell anti$M M$ response. Among all inhibitory receptors identified on an NK cell, inhibitory KIRs (iKIRs) and NKG2A receptor are often regarded as the major inhibitory receptors. While iKIRs bind to the classical HLA class I molecules, the NKG2A receptor binds to the non-classical HLA class I molecule HLA-E which is expressed by nearly all cells[104]. Although the molecular structure of HLA-E closely resembles the classical HLA class I molecules, HLA-E is far less polymorphic than the classical HLA class I molecules. Furthermore, HLA-E can only bind a restricted set of peptides, while classical HLA class I can bind and present a broad range of peptides. In chapter 4, we provided an overview on the clinical and immunological significance of HLA-E both in stem cell transplantation and in cancer. In the overview, we discussed that HLA-E presents the leader peptide of other HLA class I molecules, especially the classical HLA class I molecules (HLA-A, - B, -C) and of non-classical HLA-G. In case of downregulation of classical HLA class I molecules, as seen in many tumors as an immune evasion mechanism from CD8+ T cells killing, HLA-E often persist or its expression is even upregulated, to evade NK cell surveillance. Also in MM, we observed relatively high levels of HLA-E on primary patient cells (chapter $\mathbf{5}$ ). For these reasons, we anticipated that HLA-E-NKG2A interaction could act as an important inhibitory checkpoint for NK cells in MM.

At present, our lab is developing an NK cell-based therapy involving a large scale ex vivo expansion of peripheral blood-derived NK cells. We noticed that at the end of the expansion protocol, a large number, if not majority, of the expanded NK cells express NKG2A receptor. Apparently, the expression of NKG2A after an expansion is quite common and independent to the cytokine combinations or feeder cells used during culture $[10,37,105]$. Although this phenomenon could be just a part of normal NK cell development representing the occurrence of a high number of relatively immature NKG2A positive NK cells[106], we raised the question: should we be concerned about having an NK-cell product containing a high number of NKG2A+ NK cells, considering that some tumor cells could also upregulate HLA-E? In our previous 
study, we already demonstrated that the interaction between NKG2A and HLA-E could reduce the cytotoxic capacity of activated NK cell against MM cell lines [101]. However, the importance of HLA-E for activated NK cells remained largely elusive. Moreover, NKG2A has been widely described as an inhibitory receptor and studies suggested that it might be necessary to block the receptor using a blocking antibody (Monalizumab) [107, 108]. Therefore, we performed, in chapter 5, in-depth analysis of the inhibitory potential of NKG2A on subsets of highly activated NK cells that varied in their activation threshold due to being KIR-ligand matched/mismatched- or licensing status. Through this analysis, we showed that the expression of the NKG2A receptor was, in fact, advantageous for IL-2 activated NK cells against HLA negative tumor cells, also in the presence of a more suppressive TME factors (chapter 5). NKG2A did not inhibit IL-2 activated NK cell anti-MM activity when the levels of HLA-E expressed on the target cells were low. However, we showed that NKG2A expression can inhibit IL-2 activated NK cells anti-tumor response when the levels of HLA-E expressed on the target cells were high. This suggests that the threshold for NK-cell inhibition via NKG2A and HLA-E interaction could be higher in an activated NK-cell than in an unactivated NK-cell. An additional argument is that we used a high dose of IL-2 to pre-activate NK cells, which might also already have tipped the signaling balance towards activation, hence, stronger inhibitory signals might be required to completely abrogate NK-cell antitumor response. Therefore, blocking NKG2A-HLA-E binding using an antibody should be considered based on individual settings. 1) In case of the infusion of alloreactive highly activated ex vivo-expanded NK-cells, blocking NKG2A-HLA-E binding with an antibody might not be necessary. 2) Blocking of NKG2A-HLA-E interaction might be helpful to boost endogenous NK cells or NK cells that have reconstituted after allo-SCT, since the activation status of NK cells is not fully known and MM target cells are expressing HLA-E.

Importantly, NK-cell subsets (co-)expressing NKG2A showed better anti-MM activity compared to subsets not expressing NKG2A, regardless of their KIR expression. As NK cell licensing is a quantitative process, NK cells with a higher number of inhibitory receptors become better licensed effector cells[109] which could be an explanation for our observations. Additionally, NK cell licensing is also a qualitative process determined by the receptor-ligand interaction, therefore stronger ligands could provide higher licensing strength compared to the weaker ligands [110]. Additionally, the specificity of the peptide presented by HLA class I or HLA-E has been shown to play a role in dictating the inhibitory effect $[111,112]$ and we show in the chapter 6 that HLA expression levels could influence the inhibitory potential as well. Altogether this clearly illustrates the complexity of regulation of the NK cell anti-tumor response and the licensing process through HLA-KIR/NKG2A interaction. 
Besides inhibitory receptors binding to HLA molecules, NK cells express inhibitory receptors that have been shown to be important for regulation of $\mathrm{T}$ cell activation. Examples of these receptors are programmed cell death protein-1 (PD-1), T-cell immunoreceptor with $\mathrm{Ig}$ and immunoreceptor tyrosine-based inhibition motif domains (TIGIT), and T-cell immunoglobulin- and mucin-domain containing molecule 3 (TIM-3). While these receptors are well studied in T cells and consequently became very popular targets for intervention, their role and functional relevance in NK cells is much less studied and for some of these receptors very controversial. These receptors have been found upregulated in activated NK cells but at the same time, they also marked NK cell exhaustion and dysfunction resulting in a decreased cytotoxicity $[113,114]$. In primary MM cells, PD-1 blocking has been shown to enhance NK-cell anti-MM response specifically towards PD-L1+ MM cells by enhancing NK-cell trafficking, immune complex formation with MM cells [90]. In breast cancer cell lines, TIGIT blockade has been shown to enhance NK cells ADCC in trastuzumab-resistant tumor cells[115]. Blocking the inhibitory interaction of these receptors therefore could be a way to minimize the inhibitory signaling, however better understanding of their functional relevance for NK cells is essential as their occurrence and function for NK cells are not fully understood.

In summary, strategies to minimize inhibitory signals via NK cell receptors could be:

1) Creating KIR-ligand mismatched situation between NK-cell donor and patient, preferably, whenever possible, by selecting NK-cell donors who are genotypically HLA-C1+C2+Bw4+ and having NK cells expressing KIR2DL2/3, KIR2DL1, and KIR3DL1. Creating KIR-ligand mismatched using an anti-KIR antibody such as Lirilumab could be an alternative option when such a donor is not available. However, caution should be used as a recent study in smoldering MM pointed out that Lirilumab administration could result in NK cell hyporesponsiveness as the KIR2D molecules were cut off from NK-cell surface via trogocytosis [116].

2) Blocking other relevant inhibitory receptors. Since many of the inhibitory receptors expressed on NK cells are also involved in the education/licensing of NK cells, blocking NKG2A with antibody should be overweighed depending on the situations and we showed that its effect is highly dependent on the exact HLA-E context and NK subset. Blocking PD-1 or TIGIT receptor could be more beneficial as PD-1 and TIGIT are involved in another pathway. Nonetheless, since not all tumor cells express the ligands for PD-1 or TIGIT, it would be important to elucidate the status of the ligands expression on the targeted tumor cells. 
I can do things you cannot, you can do things I cannot; together we can do great things

\section{- Mother Teresa}

\section{Boosting NK cell activation: combination therapy with ADCC-triggering monoclonal antibody}

It takes two to tango. The discovery of new drugs has opened a limitless opportunity to treat cancers from different angles. These drugs include the immunomodulatory drugs (i.e. lenalidomide, thalidomide), monoclonal antibodies (mAbs) against tumorspecific/-associated-antigens (i.e. Rituximab, Cetuximab, Trastuzumab, Daratumumab, Elotuzumab), immune checkpoint inhibitors (i.e. anti-PD-1/PD-L1, anti-CTLA-4), and other inhibitors/blockers such as the indoleamine 2,3-dioxygenase inhibitors, the proteasome inhibitors (Bortezomib), and the KIR-blocking antibody (Lirilumab)[78]. With their own specificities and targets, these drugs could be potential combination agents for NK cell-based therapy.

In this thesis, we showed that enhancing the NK-cell antitumor response by combining IL-2 activated alloreactive NK cells with ADCC-triggering mAbs is a potential way to boost the NK cell antitumor response in the TME (chapter 2). As we mentioned earlier in the discussion, NK-cell cytotoxicity was reduced when more suppressive TME factors were present during killing process, activation NK cells with IL-2 alone was not sufficient to overcome the effect of suppressive TME (chapter 2). We demonstrated that the addition of daratumumab, an antibody against CD38 which is an antigen expressed on subsets of MM and several MM cell lines, could enhance NK-cell cytotoxicity specifically against CD38high MM cell lines (chapter $\mathbf{2}$ ). Importantly, the boosting effect of daratumumab seemed to be a general mechanism applicable to both KIR-ligand matched and KIR-ligand mismatched NK cells where an increase in the percentage of degranulating NK cells in all NK-cell subsets were observed (chapter $\mathbf{2}$ and $\mathbf{5}$ ). This finding gives a rationale for the combination of NK cell-based therapy and a mAb.

However, in this study we also learned the less desirable effect of daratumumab addition, namely NK cell-fratricide. Owing to the expression of CD38 on NK cell surface, the presence of daratumumab resulted in an increased percentage of degranulating NK cells and a higher number of dead NK cells both in the absence or presence of a target cell. This is in line with a recent study by Casneuf et al showed that daratumumab administration significantly reduced the NK-cell compartment in the 
peripheral blood of MM patients, although it did not compromise the clinical efficacy of daratumumab ADCC potential [117]. Furthermore, in patients, daratumumab has also been shown to eliminate suppressive CD38+ regulatory $T$ cells, downregulate myeloid suppressor cells as well as stimulating the expansion of helper and cytotoxic T cells [118] and inducing antibody-dependent cell-mediated phagocytosis [119]. Taking these into account, the administration of daratumumab, regardless the NK-cell fratricide, might still be beneficial to improve the elimination of MM cells. Moreover, because of the immunostimulatory capacity, the drug might also be valuable in tumors that do not express CD38 on the tumor cells. For this reason several trials are going on in solid tumors. Considering the multitude of the mechanism of actions of daratumumab, the combination of daratumumab and NK-cell might improve NKcell anti-MM response in the TME both directly and indirectly. Another alternative monoclonal antibody to be combined with NK cell-based therapy is elotuzumab, an antibody targeting CS1 (an MM-associated protein). Like daratumumab, elotuzumab has been shown to induce NK-cell mediated ADCC [120]. In addition, it provided direct NK-cell activation by triggering activating intracellular signaling pathways [121123] and via IL-2 and TNF-a pathways[124]. In addition, elotuzumab did not induce fratricide on NK-cell [121]. In the clinical studies, both antibodies have been shown to have favorable safety profile in patients with refractory or relapsed $\operatorname{MM}[125,126]$. The progression-survival rate at 12 months was $83.2 \%$ for daratumumab [127] and $68 \%$ for elotuzumab [126]. Since no clinical study investigated the combination of NK cell-based therapy and daratumumab or elotuzumab for MM yet, it would be very interesting to study the efficacy of these combinations.

In this thesis we demonstrated that combination therapy with a monoclonal antibody targeting a tumor-specific/associated antigen could provide an extra boost for the NK cell. But we also showed that the antigen specificity of the antibody and the antigen expression level of the target cell may be important. The selection of a $\mathrm{mAb}$ as combination therapy with an NK cell-based therapy should strive to select an antibody specific for antigens expressed only on the tumor cells and not on the NK cells for antibodies that also trigger NK cell-fratricide. If the targeted antigen of a $\mathrm{mAb}$ is also expressed on NK cells and induces fratricide (such as daratumumab), the method and timing to administer the two therapies should be considered to avoid the reduction of NK-cell number before reaching the tumor bed. Therefore, the selection of antibody becomes very crucial. Alternatively, when the targeted antigen is also (highly) expressed on the NK cell, in case of daratumumab, we could consider pre-treating the ex vivo-expanded NK cells with an $\mathrm{F}(\mathrm{ab})_{2}$ to mask the CD38 molecules expressed on NK cells [128]. 
In addition, bi- or tri-specific $\mathrm{mAb}$ instead of the conventional mAb can be considered to be used in the combination with NK cell-based therapy. The invention of a bi- or tri-specific killer engager (BiKE or TriKE) antibodies could help NK cells to target the tumor cells better since they are composed of a single variable portion $\left(\mathrm{V}_{\mathrm{H}}\right.$ and $V_{L}$ ) of an antibody linked to one (BiKE) or two (TriKE) variable portions from other antibodies having different specificity [129]. While a BiKE such as the anti-CD33 $x$ anti-CD16 could recognize an epitope on a tumor cell (CD33) and engages with an NK cell (CD16) simultaneously [130], a TriKE such as anti-CD33 x anti-CD16 x IL-15 crosslinker could recognize the tumor, trigger ADCC by binding CD16 on an NK cell, and provide activation/survival/expansion of an NK cell [131, 132]. However, caution should be taken when designing an antibody as a lot of antigens on tumor cells like multiple myeloma are also present on NK cells. Another important point, CD16 is known to be shed quickly from the surface upon activation by ADAM-17, a matrixmetalloproteinase [133]. Therefore, it might be of worth to also either stabilize the CD16 expression or use an ADAM-17 inhibitor.

Our lab spent a lot of time and energy by developing a bispecific antibody targeting the tumor-specific underglycosylated MUC1 and CD16. However, this was not successful possibly because the MUC1 expression on tumor cells lines was not high enough to trigger the ADCC via the antibody, more than that NK cells on their own were triggered by the tumor cells (manuscript in preparation).

Maybe you are searching among the branches, for what only appears in the roots

\section{Genetic screening for FCGR3A gene polymorphisms to boost ADCC capacity of NK cells}

The selection of the right monoclonal antibody or BiKE/TRiKE is important for combination therapy with ADCC triggering antibodies and NK cells. Another determining factor in the success of antibody therapy combination with NK cell-based therapy lies intrinsically within the NK-cell donor. Several genetic polymorphisms in the FCGR3A gene have been demonstrated to affect the strength of ADCC [134-136].

One of the most well-known polymorphism is the FcyRllla-V158F polymorphism, where there is a single nucleotide polymorphism (SNP) from G to T at CDNA nucleotide position 559 of the FCGR3A gene resulting in two different FcyRIIla allotypes: one with 
a valine $(\mathrm{V})$ and one with a phenylalanine $(\mathrm{F})$ at amino acid position 158 [136-138]. The presence of a valine (V/V or V/F) has been shown to increase the NK-cell's binding affinity to an IgG1 or IgG3 antibody [138-140] and it was associated with higher level of CD16a expression [141] as compared to the presence of a homozygous phenylalanine genotype (F/F), resulting in a higher level of NK cell-mediated ADCC and eventually a better clinical outcome, indicated by an improved progressionfree survival [142-144]. Therefore, selection of the best NK-cell donor, in addition to the KIR-ligand mismatched status, should also be based on their CD16a genotype, preferably donors with the high binding affinity (V/V) genotype. Selecting a donor with V/V genotype in combination with $\mathrm{HLA}-\mathrm{C} 1+\mathrm{C} 2+\mathrm{Bw} 4+$ genotype expressing corresponding KIRs might be sufficient to increase the NK-cell potency.

One important consideration for donor selection based on FcyRllla-V158F polymorphism is the number of such "special" donor available. In our small test panel of 76 individuals, we found that the percentage of individuals with V/V (high affinity) phenotype was the lowest compared to the other two phenotypes (V/F or F/F). In other studies, V/V frequencies, ranging between $4-23 \%$, have been reported in different populations (as reviewed in [145]). From the cancer treatment point of view, this is a rather unfortunate result, since only a small fraction of patients could maximally benefit from ADCC dependent antibody therapy. As to why V/V phenotype was always found to be the least frequent phenotype in the population, is still not known. However, as summarized in a review by Bournazos et al, the low affinity variant is usually associated with autoimmune pathologies characterized by the presence of circulating IgG complexes [146]. On the other hand, the high affinity variant is linked to chronic inflammatory disorders marked by excessive leukocyte activation. From an NK cell-donor perspective, the low frequency of high affinity genotype is not necessarily an issue since NK cells could be expanded ex vivo from a donor having the high variant or even be engineered to express a high affinity CD16a receptor although only $3-22 \%$ of the population (depending the ethnicity) are genotypically V/V [145].

To date, information on extended full length CD16a polymorphisms is still limited and, in chapter 3 of this thesis, we showed that a lot more polymorphisms exist in the FCGR3A gene than frequently described in the literature. We identified 234 polymorphisms in the FCGR3A gene with more than $85 \%$ of the polymorphisms located in the non-coding regions and $15 \%$ in the coding regions. Further analysis showed that, although most of the polymorphisms were located in the introns, a high proportion of these polymorphisms was frequently found in the tested population (2504 individuals from 26 populations) of the 1000 Genome Project. Nonetheless, 
information on functional consequence(s) of these intron polymorphisms is still lacking. A recent study on FCGR2C gene, interestingly showed that a mutation in an intronic splice site introduced novel stop codons resulting in a loss of FcyRllc expression [147]. Although the expression of FcyRllc is low on NK cells, this receptor has been shown to also mediate ADCC [148]. A loss of expression of this receptor might therefore reduce NK-cell's ADCC. Given this example and the lack of functional evidence yet, we propose that it might be of value to select some of the potential intron polymorphisms (such as SNPs located around the splice site[149]) and perform in vitro studies (and possibly in vivo mouse study) to investigate the functional consequence of these polymorphisms on CD16a receptor, especially NK cell ADCC capacity.

In addition to the lack of CD16 polymorphism data, there is currently a lack of standardized protocols, complications by interference due to high homology between FCGR3A and FCGR3b, and various methods have been employed by different research groups to study FCGR3A gene polymorphisms. In this thesis, we showed that it is feasible to identify extended polymorphisms in the FCGR3A gene, using sangerbased typing (SBT) sequencing method and MinION nanopore technology by means of a full-length FCGR3A gene sequencing. Using these two methods, we could identify polymorphisms known in the 1 KG database project as well as polymorphisms not listed in the $1 \mathrm{KG}$ database. Both methods offered the possibilities to sequence fulllength gene sequencing (although we did not obtain full length sequences by SBT yet) with their own advantages and disadvantages. Despite the high concordance between SBT and MinION, the identification of V158F polymorphism by MinION should be carefully performed as this polymorphism is located in a homopolymeric region, which is known as a problematic region for MinION and in other next generation sequencing methods [150-152]. On the other hand, a full-length gene sequencing for multiple samples was practically more efficiently done with MinION, as multiple sequencing primers were not required. Therefore, for the detection of V158F polymorphism and limited number of polymorphisms, SBT might be a more preferred approach than the MinION. However, with proper optimization and standardization, MinION could be a potential and more effective method to perform a full-length FCGR3A gene sequencing. Another group has recently demonstrated a successful high accuracy sequencing of class I HLA-gene using MinION [153]. This provides more reasons for us to consider using MinION in the future for selecting NK-cell donor. We can genotypically select an NK-cell donor based on their class I HLA-gene and KIR gene for a full alloreactivity in addition to CD16a genotype. We envisioned that MinION could be a practical and convenient tool to sequence multiple samples and multiple genes in comparison with conventional sequencing methods. 
To summarize, selecting an NK donor based on the genotypic expression of FCGR3A gene allotype could be an additional strategy to enhance NK-cell antitumor response especially in relation to ADCC response. Alternatively, CD16 polymorphism issues could also be bypassed by selecting an antibody either mono-, bi-, or trispecific mAb with an increased affinity at the Fc region and by manipulation of antibody's Fc region through fucosylation or glycosylation has been shown to increase NK-cell ADCC strength [154]. 


\section{FUTURE PERSPECTIVES}

NK cell-based immunotherapy has garnered more and more attention in the last decade. Due to its natural cancer-killing property, NK cell-based therapy has a promising future as a cell-based cancer treatment. Numerous studies have been performed by different research groups around the world, including our group, to develop, to expand and refine NK cells and to use them for NK cell-based therapy [155]. Notwithstanding the potential and the feasibility of NK cell-based therapy, there is still considerable room for the improvement of its clinical efficacy. In this thesis, we highlighted the potential challenges that NK cells might encounter in the tumor microenvironment and proposed several strategies to improve NK cell antitumor response.

Besides the strategies we discussed in this thesis, other strategies that we could foresee as potential methods to improve NK cell-based therapy are:

\section{Improving NK-cell survival in- and NK-cell trafficking to the tumor site in vivo.}

As previously mentioned in this thesis, NK-cell survival in- and trafficking to the tumor bed due to suppressive TME are often the issue for NK cell-based therapy. Since NK cell survival is dependent on certain cytokines [156], the administration of an immunocytokine, for example, might be able to provide a survival and or trafficking signal for the NK cell in vivo. An immunocytokine is an antibody targeting a tumor-specific/associated antigen fused to a cytokine [157]. L19-IL2 (Darleukin), is an immunocytokine which consist of an antibody L19 targeting the extra domain $B$ (ED-B) of fibronectin and IL-2. A previous study demonstrated that L19-IL2 could localize to the tumor tissue expressing ED-B and more importantly, it could activate NK cells which led to the eradication of tumor cells [158]. Currently, several immunocytokines are available and their clinical efficacy is being investigated in different clinical trials [157]. Since IL-2 is not the only cytokine that is important for NK cell survival, an immunocytokine consisting another cytokine such as IL-15 could be a potential cytokine as well. Additionally, a chemokine could probably be utilized instead of a cytokine with the purpose to improve NK cell trafficking. Alternatively, an improvement of human NK-cell survival or persistence in vivo by culturing NK cells with PM21 particles [36] or with K562 feeder cells expressing membrane-bound IL-21 (K562-mb-IL-21) [159] possibly because NK-cells expanded with either PM21 or K562$\mathrm{mb}-\mathrm{IL} 21$ have an increase in the telomere length and less senescence [160]. 


\section{Generating a large number of highly activated NK cells.}

One of the major hurdles in NK cell-based therapy is the insufficient number of NK cells infused to the patient. Several expansion protocols, including clinical-grade protocols, have been developed to cultivate NK cells ex vivo [155]. Fully automated expansion and activation protocol of clinical-grade NK cells have been developed by several groups, yielding up to $19 \times 10^{9}$ NK cells[29, 31, 34, 161]. Although these numbers are enough for a typical dose used for 1 patient in the current NK cell-based therapy, the optimal dose of NK cell injection is still needs to be elucidated. A clinical study on high risk relapsed MM patients has demonstrated the safety of a dose up to $1 \times 10^{8}$ activated ex vivo-expanded NK cells/kg per patient [162]. It is however not unlikely that a higher dose would be needed in the future. Hence, multiple expansion runs would be required in this protocol, which might give a batch-to-batch variation. Therefore, alternative protocols/methods to maximize NK cell expansion still needs to be further investigated.

\section{Investigating the metabolic profile of NK cells.}

Accumulating evidence on the influences of TME on the metabolism of cytotoxic T cells has shed a new light on strategies to improve T-cell based therapy. We showed that unlike CD8+ T cells, NK cells did not seem to require high glucose concentration during culture or to become activated. It would be very attractive to study the metabolic profile of NK cells especially in the context of TME. This is not only relevant to optimize the ex vivo culture condition of NK cells but also to study whether certain activating or inhibitory pathways are regulated by in particular metabolism and vice versa [163]. Dissection of the metabolic pathways of NK cells would open the path to the possible ex vivo metabolic interventions to create potent NK cells.

\section{Increasing activating signaling and antigen specificity by engineering ultra-potent chimeric antigen receptor (CAR)-NK cells.}

The advances in molecular manipulation have made it feasible to create T-cells with enhanced targeting and activation towards tumor cells. Using similar principles, NK cells can also be engineered to better target tumor cells. A CAR consists of an external recognition domain (usually a small chain variable fragment) directed at a tumor antigen that is linked with one or more intracellular signaling domains that mediate T- or NK-cell activation. Different groups used either NK-92 cell lines or primary NK cells derived from the PBMC to engineer CAR-NK cells targeting different tumor antigens (reviewed in [164]). The use of NK-92 cell line or primary NK cells has its own advantages and disadvantages. While NK-92 cell line is easier to transduce, since it is principally a malignant NK cell line, these NK cells express fewer receptors than the primary NK cells. Additionally, the CAR product needs to be irradiated prior to infusion for safety reason therefore limiting its 
efficacy and persistence in vivo. On the other hand, the major hindrance with primary NK cells is that these cells are more difficult to transduce [165]. If this issue can be solved, CARNK cells could hold the future of NK cell-based therapy, since it might also offer several advantages over CAR-T cells, such as a shorter life span than T cells therefore eliminating the need of the incorporation of a suicide gene, less risk of "off-target" cytotoxicity as well as cytotoxicity to normal tissues (GvHD), and less risk in inducing cytokine storm [15, 166].

\section{Combination of haploidentical transplantation, antibodies and ex vivo- expanded NK cells in MM.}

Since haplo-SCT recently starts to become a routine procedure to treat patients with hematological malignancies, combining haplo-SCT with (haploidentical) ex vivoexpanded NK cells could be a promising combination. Proceeding the haplo-SCT, the reconstitution of NK cells from the donor takes approximately two months [167], therefore giving the window to administer ex vivo-expanded NK cells. The combination of haplo-SCT and (haploidentical) ex vivo-expanded NK cells offers several advantages for the patient. 1) The process of donor selection for both procedures needs to be done only once, 2 ) due to a delayed in immune reconstitution, ex vivo-expanded NK cells could minimalize the risk of infection and relapse after SCT, and 3) increase the GvL effect without aggravating GvHD. In addition, based on the results presented in this thesis patients will also be treated with Elotuzumab, an antibody that activates the NK cells activity against MM. Our first model in haploidentical transplantation in mice was in breast cancer, with good anti-tumor activity by donor NK cells. Since a substantial group of patients with breast cancer will still die because of the disease we consider the concept of haploidentical transplantation and NK cells a valuable concept to be explored in breast cancer as well. Clinical studies will be developed as soon as the procedure is feasible in $M M$. Since it is already feasible in AML we consider this a realistic goal.

Given that there are several options on how we can use an NK cell-based therapy and more options on how we can further enhance it, the one question remains is: How to use these NK cells?

If you know your enemies and know yourself, you will not be imperiled in a hundred battles; if you do not know your enemies but do know yourself, you will win one and lose one; if you do not know your enemies nor yourself, you will be imperiled in every single battle. 
In this thesis we showed that (cytokine-) activated alloreactive KIR-ligand mismatched NK cells are the better effector cells compared to the KIR-ligand matched NK cells against different (hematological) tumor cells. Therefore, the first consideration would be checking the HLA genotype of a patient and the status HLA expression on the target cells as well as checking the status of NK cell donor's genotype and phenotype. Tumors expressing an intact HLA-C1, C2, and Bw4 molecules might benefit less from an NK cell-based therapy, and might even benefit more from a T-cell based therapy. Otherwise, anti-KIR antibody might be used to help to create KIR-ligand mismatch.

Second, an NK cell-based therapy can be both administered as a single donor NK-cell infusion or in addition to a HaploSCT. In patients with hematological malignancies, AutoSCT or HLA-identical AlloSCT is a standard care for a certain group of patients (mostly younger patients). However, since haplo-SCT becomes more routinely performed in patients with myeloid malignancies, lymphoid malignancies, as well as solid tumors[23], it becomes more realistic to administer ex vivo-expanded NK cells following haplo-SCT. However, for patients who could not withstand the conditioning therapy prior to the haplo-SCT due to older age or unfit for the therapy, infusion of ex vivo-expanded NK cells could be given without the combination with haplo-SCT. Third, personalization of combination therapy. Considering the infinite choice of therapies to combine with an NK cell-based therapy (mAb, inhibitors/blockers, immunomodulatory drugs, immunocytokines, etc), a decision should consider the type, characteristic, heterogeneity of the cancer, the TME, as well as patient's condition. This is not only to avoid a predictable negative outcome, but also to avoid unnecessary expensive treatment cost and to maximize the combination therapy. Therefore, investigation on specific biomarkers or other pathologic examinations should be done prior to therapy selection for better prediction.

Finally, we hope that this thesis could stress the importance of TME as a major threat of an NK cell-based immunotherapy. More importantly, we hope that this thesis could provide more insights and ideas on how we can exploit alloreactive NK cells to unleash its full capacity as the natural-born tumor-cell killer in such suppressive conditions. 


\section{REFERENCES}

1. Kazandjian D (2016) Multiple myeloma epidemiology and survival: A unique malignancy. Semin Oncol 43:676-681. doi: 10.1053/j.seminoncol.2016.11.004

2. Rajkumar SV, Kumar S (2016) Multiple Myeloma: Diagnosis and Treatment. Mayo Clin Proc 91:101-119. doi: 10.1016/j.mayocp.2015.11.007

3. Lonial S (2010) Relapsed multiple myeloma. Hematology Am Soc Hematol Educ Program 2010:303-9. doi: 10.1182/asheducation-2010.1.303

4. Chan Chung KC, Tiedemann RE (2014) Getting to the root of the problem: The causes of relapse in multiple myeloma. Expert Rev Anticancer Ther 14:251-254. doi: 10.1586/14737140.2014.868776

5. Nicholson SE, Keating N, Belz GT (2017) Natural killer cells and anti-tumor immunity. Mol Immunol 0-1. doi: 10.1016/j.molimm.2017.12.002

6. Rezvani K, Rouce RH (2015) The application of natural killer cell immunotherapy for the treatment of cancer. Front Immunol. doi: 10.3389/fimmu.2015.00578

7. Szmania S, Lapteva N, Garg T, et al (2015) Ex Vivo-expanded Natural Killer Cells Demonstrate Robust Proliferation In Vivo in High-risk Relapsed Multiple Myeloma Patients. J Immunother 38:24-36. doi: 10.1097/CJI.0000000000000059

8. Geller M a, Cooley S, Judson PL, et al (2011) A phase II study of allogeneic natural killer cell therapy to treat patients with recurrent ovarian and breast cancer. Cytotherapy 13:98-107. doi: $10.3109 / 14653249.2010 .515582$

9. Berg M, Lundqvist A, Jr PM, et al (2009) Clinical Grade Ex Vivo-Expanded Human Natural Killer Cells Upregulate Activating Receptors and Death Receptor Ligands and Have Enhanced Cytolytic Activity against Tumor Cells. Cytotherapy 11:341-355. doi: 10.1080/14653240902807034. Clinical

10. Granzin M, Wagner J, Köhl U, et al (2017) Shaping of natural killer cell antitumor activity by ex vivo cultivation. Front Immunol. doi: 10.3389/fimmu.2017.00458

11. Koehl U, Brehm C, Huenecke S, et al (2013) Clinical Grade Purification and Expansion of NK Cell Products for an Optimized Manufacturing Protocol. Front Oncol 3:1-12. doi: 10.3389/ fonc. 2013.00118

12. Lapteva N, Szmania SM, van Rhee F, Rooney CM (2014) Clinical grade purification and expansion of natural killer cells. Crit Rev Oncog 19:121-32. doi: 10.1109/TMI.2012.2196707.Separate

13. Zhang C, Oberoi P, Oelsner S, et al (2017) Chimeric antigen receptor-engineered NK-92 cells: An off-the-shelf cellular therapeutic for targeted elimination of cancer cells and induction of protective antitumor immunity. Front Immunol. doi: 10.3389/fimmu.2017.00533

14. Liu D, Tian S, Zhang K, et al (2017) Chimeric antigen receptor (CAR)-modified natural killer cellbased immunotherapy and immunological synapse formation in cancer and HIV. Protein Cell 8:861-877. doi: 10.1007/s13238-017-0415-5

15. Glienke W, Esser R, Priesner C, et al (2015) Advantages and applications of CAR-expressing natural killer cells. Front Pharmacol 6:1-7. doi: 10.3389/fphar.2015.00021

16. Patriarca $\mathrm{F}$, Einsele $\mathrm{H}$, Spina $\mathrm{F}$, et al (2012) Allogeneic stem cell transplantation in multiple myeloma relapsed after autograft: A multicenter retrospective study based on donor availability. Biol Blood Marrow Transplant 18:617-626. doi: 10.1016/j.bbmt.2011.07.026

17. Passera R, Pollichieni S, Brunello L, et al (2013) Allogeneic hematopoietic cell transplantation from unrelated donors in multiple myeloma: Study from the italian bone marrow donor registry. Biol Blood Marrow Transplant 19:940-948. doi: 10.1016/j.bbmt.2013.03.012

18. Mir MA, Kapoor P, Kumar S, et al (2015) Trends and outcomes in allogeneic hematopoietic stem cell transplant for multiple myeloma at Mayo Clinic. Clin Lymphoma, Myeloma Leuk 15:349357.e2. doi: 10.1016/j.clml.2015.03.016 
19. Dhakal B, Vesole DH, Hari PN (2016) Allogeneic stem cell transplantation for multiple myeloma: is there a future? Bone Marrow Transplant 51:492-500. doi: 10.1038/bmt.2015.325

20. Giralt S, Koehne G (2013) Allogeneic hematopoietic stem cell transplantation for multiple myeloma: What place, if any? Curr Hematol Malig Rep 8:284-290. doi: 10.1007/s11899-0130185-y

21. Ruggeri L, Capanni M, Urbani E, et al (2002) Effectiveness of Donor Natural Killer Cell Alloreactivity in Mismatched Hematopoietic Transplants. Science (80- ) 295:2097-2100. doi: 10.1126/science. 1068440

22. Frings PWH, Van Elssen CHMJ, Wieten L, et al (2011) Elimination of the chemotherapy resistant subpopulation of 4T1 mouse breast cancer by haploidentical NK cells cures the vast majority of mice. Breast Cancer Res Treat 130:773-781. doi: 10.1007/s10549-011-1355-z

23. Passweg JR, Baldomero $\mathrm{H}$, Bader $\mathrm{P}$, et al (2017) Use of haploidentical stem cell transplantation continues to increase: The 2015 European Society for Blood and Marrow Transplant activity survey report. Bone Marrow Transplant 52:811-817. doi: 10.1038/bmt.2017.34

24. Sun Y-Q, Chang Y-J, Huang X-J (2018) Update on current research into haploidentical hematopoietic stem cell transplantation. Expert Rev Hematol 11:273-284. doi: 10.1080/17474086.2018.1447379

25. Tonn T, Schwabe D, Klingemann HG, et al (2013) Treatment of patients with advanced cancer with the natural killer cell line NK-92. Cytotherapy 15:1563-1570. doi: 10.1016/j.jcyt.2013.06.017

26. Spanholtz J, Tordoir M, Eissens D, et al (2010) High log-scale expansion of functional human natural killer cells from umbilical cord blood CD34-positive cells for adoptive cancer immunotherapy. PLoS One. doi: 10.1371/journal.pone.0009221

27. Spanholtz J, Preijers F, Tordoir M, et al (2011) Clinical-grade generation of active NK cells from cord blood hematopoietic progenitor cells for immunotherapy using a closed-system culture process. PLoS One. doi: 10.1371/journal.pone.0020740

28. Cany J, Dolstra H, Shah N (2015) Umbilical cord blood-derived cellular products for cancer immunotherapy. Cytotherapy 17:739-748. doi: 10.1016/j.jcyt.2015.03.005

29. Sutlu T, Stellan B, Gilljam M, et al (2010) Clinical-grade, large-scale, feeder-free expansion of highly active human natural killer cells for adoptive immunotherapy using an automated bioreactor. Cytotherapy 12:1044-1055. doi: 10.3109/14653249.2010.504770

30. Lee HR, Son $\mathrm{CH}$, Koh EK, et al (2017) Expansion of cytotoxic natural killer cells using irradiated autologous peripheral blood mononuclear cells and anti-CD16 antibody. Sci Rep 7:1-13. doi: 10.1038/s41598-017-09259-1

31. Granzin M, Soltenborn S, Müller S, et al (2015) Fully automated expansion and activation of clinical-grade natural killer cells for adoptive immunotherapy. Cytotherapy 17:621-632. doi: 10.1016/j.jcyt.2015.03.611

32. Phan MT, Lee S, Kim S, Cho D (2016) Natural Killer Cells. 1441:167-174. doi: 10.1007/978-1-49393684-7

33. Bae DS, Lee JK (2014) Development of NK cell expansion methods using feeder cells from human myelogenous leukemia cell line. Blood Res 49:154-161. doi: 10.5045/br.2014.49.3.154

34. Klöß S, Oberschmidt O, Morgan M, et al (2017) Optimization of Human NK Cell Manufacturing: Fully Automated Separation, Improved Ex Vivo Expansion Using IL-21 with Autologous Feeder Cells, and Generation of Anti-CD123-CAR-Expressing Effector Cells. Hum Gene Ther 28:897-913. doi: 10.1089/hum.2017.157

35. Oyer JL, Igarashi RY, Kulikowski AR, et al (2015) Generation of highly cytotoxic natural killer cells for treatment of acute myelogenous leukemia using a feeder-free, particle-based approach. Biol Blood Marrow Transplant 21:632-639. doi: 10.1016/j.bbmt.2014.12.037

36. Oyer JL, Pandey V, Igarashi RY, et al (2016) Natural killer cells stimulated with PM21 particles expand and biodistribute in vivo: Clinical implications for cancer treatment. Cytotherapy 18:653-663. doi: 10.1016/j.jcyt.2016.02.006 
37. Wagner J, Pfannenstiel V, Waldmann A, et al (2017) A two-phase expansion protocol combining interleukin (IL)-15 and IL-21 improves natural killer cell proliferation and cytotoxicity against rhabdomyosarcoma. Front Immunol. doi: 10.3389/fimmu.2017.00676

38. Masuyama J ichi, Murakami T, Iwamoto S, Fujita S (2016) Ex vivo expansion of natural killer cells from human peripheral blood mononuclear cells co-stimulated with anti-CD3 and anti-CD52 monoclonal antibodies. Cytotherapy 18:80-90. doi: 10.1016/j.jcyt.2015.09.011

39. Carlens S, Gilljam M, Chambers BJ, et al (2001) A new method for in vitro expansion of cytotoxic human CD3-CD56+ natural killer cells. Hum Immunol 62:1092-1098. doi: 10.1016/S01988859(01)00313-5

40. Koehl U, Kalberer C, Spanholtz J, et al (2016) Advances in clinical NK cell studies: Donor selection, manufacturing and quality control. Oncoimmunology 5:1-11. doi: 10.1080/2162402X.2015.1115178

41. Wang M, Zhao J, Zhang L, et al (2017) Role of tumor microenvironment in tumorigenesis. J Cancer 8:761-773. doi: 10.7150/jca.17648

42. Vitale M, Cantoni C, Pietra G, et al (2014) Effect of tumor cells and tumor microenvironment on NK-cell function. Eur J Immunol 44:1582-1592. doi: 10.1002/eji.201344272

43. Balsamo M, Scordamaglia F, Pietra G, et al (2009) Melanoma-associated fibroblasts modulate NK cell phenotype and antitumor cytotoxicity. Proc Natl Acad Sci 106:20847-20852. doi: 10.1073/ pnas.0906481106

44. Li T, Yang Y, Hua X, et al (2012) Hepatocellular carcinoma-associated fibroblasts trigger NK cell dysfunction via PGE2 and IDO. Cancer Lett 318:154-161. doi: 10.1016/j.canlet.2011.12.020

45. Li T, Yi S, Liu W, et al (2013) Colorectal carcinoma-derived fibroblasts modulate natural killer cell phenotype and antitumor cytotoxicity. Med Oncol. doi: 10.1007/s12032-013-0663-z

46. Sprinzl MF, Reisinger F, Puschnik A, et al (2013) Sorafenib perpetuates cellular anticancer effector functions by modulating the crosstalk between macrophages and natural killer cells. Hepatology 57:2358-2368. doi: 10.1002/hep.26328

47. Hoechst B, Voigtlaender T, Ormandy L, et al (2009) Myeloid derived suppressor cells inhibit natural killer cells in patients with hepatocellular carcinoma via the NKp30 receptor. Hepatology 50:799-807. doi: 10.1002/hep.23054

48. Ghiringhelli F, Ménard C, Terme M, et al (2005) CD4 + CD25 + regulatory T cells inhibit natural killer cell functions in a transforming growth factor- $\beta$-dependent manner. J Exp Med 202:10751085. doi: $10.1084 /$ jem.20051511

49. Cretu A, Brooks PC (2007) Impact of the non-cellular tumor microenvironment on metastasis: Potential therapeutic and imaging opportunities. J Cell Physiol 213:391-402. doi: 10.1002/ jcp. 21222

50. Lyssiotis CA, Kimmelman AC (2017) Metabolic Interactions in the Tumor Microenvironment. Trends Cell Biol 27:863-875. doi: 10.1016/j.tcb.2017.06.003

51. Lokshin A, Raskovalova T, Huang X, et al (2006) Adenosine-mediated inhibition of the cytotoxic activity and cytokine production by activated natural killer cells. Cancer Res 66:7758-7765. doi: 10.1158/0008-5472.CAN-06-0478

52. Bottino C, Chiesa M Della, Dondero A, et al (2017) Overview of the Soluble and Membranebound Tumor Factors Limiting NKmediated Immune Surveillance. Immunother Open Access 03:1-8. doi: 10.4172/2471-9552.1000136

53. Holt D, Ma X, Kundu N, Fulton A (2011) Prostaglandin E2 (PGE2) suppresses natural killer cell function primarily through the PGE2 receptor EP4. Cancer Immunol Immunother 60:1577-1586. doi: 10.1007/s00262-011-1064-9

54. Scott KEN, Cleveland JL (2016) Lactate Wreaks Havoc on Tumor-Infiltrating T and NK Cells. Cell Metab 24:649-650. doi: 10.1016/j.cmet.2016.10.015 
55. Husain Z, Huang Y, Seth P, Sukhatme VP (2013) Tumor-Derived Lactate Modifies Antitumor Immune Response: Effect on Myeloid-Derived Suppressor Cells and NK Cells. J Immunol 191:1486-1495. doi: 10.4049/jimmunol.1202702

56. Stiff A, Trikha P, Mundy-Bosse BL, et al (2018) Nitric Oxide Production by Myeloid Derived Suppressor Cells Plays a Role in Impairing Fc Receptor-Mediated Natural Killer Cell Function. Clin Cancer Res clincanres.0691.2017. doi: 10.1158/1078-0432.CCR-17-0691

57. Fujisaki H, Kakuda H, Shimasaki N, et al (2009) Expansion of highly cytotoxic human natural killer cells for cancer cell therapy. Cancer Res 69:4010-4017. doi: 10.1158/0008-5472.CAN-08-3712

58. Frohn C, Höppner M, Schlenke P, et al (2002) Anti-myeloma activity of natural killer lymphocytes. Br J Haematol 119:660-664. doi: 10.1046/j.1365-2141.2002.03879.x

59. von Strandmann EP (2006) A novel bispecific protein (ULBP2-BB4) targeting the NKG2D receptor on natural killer (NK) cells and CD138 activates NK cells and has potent antitumor activity against human multiple myeloma in vitro and in vivo. Blood 107:1955-1962. doi: 10.1182/ blood-2005-05-2177

60. Liu LL, Béziat V, Oei VYS, et al (2017) Ex Vivo-Expanded Adaptive NK Cells Effectively Kill Primary Acute Lymphoblastic Leukemia Cells. Cancer Immunol Res 5:654-665. doi: 10.1158/2326-6066. CIR-16-0296

61. Igarashi T, Wynberg J, Srinivasan R, et al (2004) Enhanced cytotoxicity of allogeneic NK cells with killer immunoglobulin-like receptor ligand incompatibility against melanoma and renal cell carcinoma cells. Blood 104:170-7. doi: 10.1182/blood-2003-12-4438

62. Kim H-R, Park H-J, Park JH, et al (2004) Characteristics of the killing mechanism of human natural killer cells against hepatocellular carcinoma cell lines HepG2 and Hep3B. Cancer Immunol Immunother 53:461-470. doi: 10.1007/s00262-003-0461-0

63. Re F, Staudacher C, Zamai L, et al (2006) Killer cell Ig-like receptors ligand-mismatched, alloreactive natural killer cells lyse primary solid tumors. Cancer 107:640-648. doi: 10.1002/ cncr.22002

64. Castriconi R, Dondero A, Negri F, et al (2007) Both CD133+ and CD133- medulloblastoma cell lines express ligands for triggering NK receptors and are susceptible to NK-mediated cytotoxicity. Eur J Immunol 37:3190-3196. doi: 10.1002/eji.200737546

65. Pietra G, Manzini C, Vitale M, et al (2009) Natural killer cells kill human melanoma cells with characteristics of cancer stem cells. Int Immunol 21:793-801. doi: 10.1093/intimm/dxp047

66. Guo M, Wu T, Wan L (2016) Cytotoxic activity of allogeneic natural killer cells on U251 glioma cells in vitro. 583-589. doi: 10.3892/mmr.2016.5220

67. Bachanova V, Sarhan D, DeFor TE, et al (2017) Haploidentical natural killer cells induce remissions in non-Hodgkin lymphoma patients with low levels of immune-suppressor cells. Cancer Immunol Immunother 67:1-12. doi: 10.1007/s00262-017-2100-1

68. Björklund AT, Carlsten M, Sohlberg E, et al (2018) Complete Remission with Reduction of Highrisk Clones following Haploidentical NK Cell Therapy against MDS and AML. Clin Cancer Res clincanres.3196.2017. doi: 10.1158/1078-0432.CCR-17-3196

69. Curti A, Ruggeri L, Addio AD, et al (2011) Successful transfer of alloreactive haploidentical KIR ligand-mismatched natural killer cells after infusion in elderly high risk acute myeloid leukemia patients Successful transfer of alloreactive haploidentical KIR ligand-mismatched natural killer cells. Blood 118:3273-9. doi: 10.1182/blood-2011-01-329508

70. Murray S, Lundqvist A (2016) Targeting the tumor microenvironment to improve natural killer cell-based immunotherapies: On being in the right place at the right time, with resilience. Hum Vaccines Immunother 12:607-611. doi: 10.1080/21645515.2015.1096458

71. Manier S, Sacco A, Leleu X, et al (2012) Bone marrow microenvironment in multiple myeloma progression. J Biomed Biotechnol. doi: 10.1155/2012/157496

72. Eliasson P, Jönsson J-I (2010) The hematopoietic stem cell niche: Low in oxygen but a nice place to be. J Cell Physiol 222:17-22. doi: 10.1002/jcp.21908 
73. Nakagawa $\mathrm{Y}$, Ashihara $\mathrm{E}$, Yao $\mathrm{H}$, et al (2018) Multiple myeloma cells adapted to long-exposure of hypoxia exhibit stem cell characters with TGF- $\beta /$ Smad pathway activation. Biochem Biophys Res Commun 496:490-496. doi: 10.1016/j.bbrc.2018.01.034

74. Braga WMT, da Silva BR, de Carvalho AC, et al (2014) FOXP3 and CTLA4 overexpression in multiple myeloma bone marrow as a sign of accumulation of CD4(+) T regulatory cells. Cancer Immunol Immunother 63:1189-97. doi: 10.1007/s00262-014-1589-9

75. Bryant C, Suen H, Brown R, et al (2013) Long-term survival in multiple myeloma is associated with a distinct immunological profile, which includes proliferative cytotoxic T-cell clones and a favourable Treg/Th17 balance. Blood Cancer J 3:1-7. doi: 10.1038/bcj.2013.34

76. Giallongo C, Tibullo D, Parrinello NL, et al (2016) Granulocyte-like myeloid derived suppressor cells (G-MDSC) are increased in multiple myeloma and are driven by dysfunctional mesenchymal stem cells (MSC). Oncotarget 7:85764-85775. doi: 10.18632/oncotarget.7969

77. Zheng Y, Cai Z, Wang S, et al (2009) Macrophages are an abundant component of myeloma microenvironment and protect myeloma cells from chemotherapy drug-induced apoptosis. Blood 114:3625-8. doi: 10.1182/blood-2009-05-220285

78. Pittari G, Vago L, Festuccia M, et al (2017) Restoring natural killer cell immunity against multiple myeloma in the era of new drugs. Front Immunol 8:1-19. doi: 10.3389/fimmu.2017.01444

79. Meads MB, Hazlehurst LA, Dalton WS (2008) The bone marrow microenvironment as a tumor sanctuary and contributor to drug resistance. Clin Cancer Res 14:2519-2526. doi: 10.1158/10780432.CCR-07-2223

80. Fujiwara S, Kawano Y, Yuki H, et al (2013) PDK1 inhibition is a novel therapeutic target in multiple myeloma. Br J Cancer 108:170-8. doi: 10.1038/bjc.2012.527

81. Sarkar S, Germeraad WT V, Rouschop KMA, et al (2013) Hypoxia induced impairment of NK cell cytotoxicity against multiple myeloma can be overcome by IL-2 activation of the NK cells. PLoS One 8:e64835. doi: 10.1371/journal.pone.0064835

82. Lunt SY, Vander Heiden MG (2011) Aerobic Glycolysis: Meeting the Metabolic Requirements of Cell Proliferation. Annu Rev Cell Dev Biol 27:441-464. doi: 10.1146/annurev-cellbio-092910-154237

83. Buck MD, Sowell RT, Kaech SM, Pearce EL (2017) Metabolic Instruction of Immunity. Cell 169:570-586. doi: 10.1016/j.cell.2017.04.004

84. Chen M, Zheng H, Wei T, et al (2016) High glucose-induced PC12 cell death by increasing glutamate production and decreasing methyl group metabolism. Biomed Res Int. doi: $10.1155 / 2016 / 4125731$

85. Peiró C, Lafuente N, Matesanz N, et al (2001) High glucose induces cell death of cultured human aortic smooth muscle cells through the formation of hydrogen peroxide. $\mathrm{Br} J$ Pharmacol 133:967-74. doi: 10.1038/sj.bjp.0704184

86. Smart EJ, Li XA (2007) Hyperglycemia: Cell death in a cave. Biochim Biophys Acta - Mol Basis Dis 1772:524-526. doi: 10.1016/j.bbadis.2007.01.005

87. Stojanovic A, Cerwenka A (2011) Natural killer cells and solid tumors. J Innate Immun 3:355-364. doi: 10.1159/000325465

88. Fauriat C, Mallet F, Olive D, costello RT (2006) Impaired activating receptor expression pattern in natural killer cells from patients with multiple myeloma. Leukemia 20:732-733. doi: 10.1038/ sj.leu.2404096

89. Costello RT, Boehrer A, Sanchez C, et al (2013) Differential expression of natural killer cell activating receptors in blood versus bone marrow in patients with monoclonal gammopathy. Immunology 139:338-341. doi: 10.1111/imm.12082

90. Jr Benson DM, Bakan CE, Mishra A, et al (2010) The PD-1 / PD-L1 axis modulates the natural killer cell versus multiple myeloma effect : a therapeutic target for CT-011, a novel monoclonal anti PD-1 antibody. Blood 116:2286-2294. doi: 10.1182/blood-2010-02-271874.The 
91. Cantoni C, Huergo-Zapico L, Parodi M, et al (2016) NK Cells, Tumor Cell Transition, and Tumor Progression in Solid Malignancies: New Hints for NK-Based Immunotherapy? J Immunol Res. doi: 10.1155/2016/4684268

92. Ponzetta A, Benigni G, Antonangeli F, et al (2015) Multiple myeloma impairs bone marrow localization of effector natural killer cells by altering the chemokine microenvironment. Cancer Res 75:4766-4777. doi: 10.1158/0008-5472.CAN-15-1320

93. Garg TK, Szmania SM, Khan JA, et al (2012) Highly activated and expanded natural killer cells for multiple myeloma immunotherapy. Haematologica 97:1348-1356. doi: 10.3324/ haematol.2011.056747

94. Heindl A, Nawaz S, Yuan Y (2015) Mapping spatial heterogeneity in the tumor microenvironment: A new era for digital pathology. Lab Investig 95:377-384. doi: 10.1038/labinvest.2014.155

95. Lee SSY, Bindokas VP, Kron SJ (2017) Multiplex three-dimensional optical mapping of tumor immune microenvironment. Sci Rep 7:1-11. doi: 10.1038/s41598-017-16987-x

96. Katt ME, Placone AL, Wong AD, et al (2016) In Vitro Tumor Models: Advantages, Disadvantages, Variables, and Selecting the Right Platform. Front Bioeng Biotechnol. doi: 10.3389/ fbioe.2016.00012

97. Zanoni M, Piccinini F, Arienti C, et al (2016) 3D tumor spheroid models for in vitro therapeutic screening: A systematic approach to enhance the biological relevance of data obtained. Sci Rep 6:1-11. doi: 10.1038/srep19103

98. Lanier LL (2005) Nk Cell Recognition. Annu Rev Immunol 23:225-274. doi: 10.1146/annurev. immunol.23.021704.115526

99. Bi J, Tian Z (2017) NK cell exhaustion. Front Immunol. doi: 10.3389/fimmu.2017.00760

100. Hicklin DJ, Marincola FM, Ferrone S (1999) HLA class I antigen downregulation in human cancers: T-cell immunotherapy revives an old story. Mol Med Today 5:178-186. doi: 10.1016/ S1357-4310(99)01451-3

101. Sarkar S, van Gelder M, Noort W, et al (2015) Optimal selection of natural killer cells to kill myeloma: the role of HLA-E and NKG2A. Cancer Immunol Immunother 64:951-963. doi: 10.1007/s00262-015-1694-4

102. Gao M, Gao L, Yang G, et al (2014) Myeloma cells resistance to NK cell lysis mainly involves an HLA class I-dependent mechanism. Acta Biochim Biophys Sin (Shanghai) 46:597-604. doi: 10.1093/abbs/gmu041

103. Martínez-Sánchez M V., Periago A, Legaz I, et al (2016) Overexpression of KIR inhibitory ligands (HLA-I) determines that immunosurveillance of myeloma depends on diverse and strong NK cell licensing. Oncoimmunology 5:1-12. doi: 10.1080/2162402X.2015.1093721

104. Lee N, Llano M, Carretero M, et al (1998) HLA-E is a major ligand for the natural killer inhibitory receptor CD94/NKG2A. Proc Natl Acad Sci 95:5199-5204. doi: 10.1073/pnas.95.9.5199

105. Suck G, Koh MBC (2010) Emerging natural killer cell immunotherapies: Large-scale ex vivo production of highly potent anticancer effectors. Hematol Oncol Stem Cell Ther 3:135-142. doi: 10.1016/S1658-3876(10)50024-4

106. Freud AG, Caligiuri MA (2006) Human natural killer cell development. Immunol Rev 214:56-72. doi: 10.1111/j.1600-065X.2006.00451.x

107. Ruggeri L, Urbani E, Andre P, et al (2016) Effects of anti-NKG2A antibody administration on leukemia and normal hematopoietic cells. Haematologica 101:626-633. doi: 10.3324/ haematol.2015.135301

108. McWilliams EM, Mele JM, Cheney C, et al (2016) Therapeutic CD94/NKG2A blockade improves natural killer cell dysfunction in chronic lymphocytic leukemia. Oncoimmunology 5:1-9. doi: 10.1080/2162402X.2016.1226720

109. Thomas LM (2015) Current perspectives on natural killer cell education and tolerance: emerging roles for inhibitory receptors. Immunotargets Ther 4:45-53. doi: 10.2147/ITT.S61498 
110. He Y, Tian Z (2017) NK cell education via nonclassical MHC and non-MHC ligands. Cell Mol Immunol 14:321-330. doi: 10.1038/cmi.2016.26

111. Das J, Khakoo SI (2015) NK cells: Tuned by peptide? Immunol Rev 267:214-227. doi: 10.1111/ imr.12315

112. Cheent KS, Jamil KM, Cassidy S, et al (2013) Synergistic inhibition of natural killer cells by the nonsignaling molecule CD94. Proc Natl Acad Sci 110:16981-16986. doi: 10.1073/ pnas. 1304366110

113. Liu Y, Cheng Y, Xu Y, et al (2017) Increased expression of programmed cell death protein 1 on NK cells inhibits NK-cell-mediated anti-tumor function and indicates poor prognosis in digestive cancers. Oncogene 36:6143-6153. doi: 10.1038/onc.2017.209

114. Anderson AC, Joller N, Kuchroo VK (2016) Lag-3, Tim-3, and TIGIT: Co-inhibitory Receptors with Specialized Functions in Immune Regulation. Immunity 44:989-1004. doi: 10.1016/j. immuni.2016.05.001

115. Xu F, Sunderland A, Zhou Y, et al (2017) Blockade of CD112R and TIGIT signaling sensitizes human natural killer cell functions. Cancer Immunol Immunother 66:1367-1375. doi: 10.1007/ s00262-017-2031-x

116. Carlsten M, Korde N, Kotecha R, et al (2016) Checkpoint inhibition of KIR2D with the monoclonal antibody IPH2101 induces contraction and hyporesponsiveness of NK cells in patients with myeloma. Clin Cancer Res 22:5211-5222. doi: 10.1158/1078-0432.CCR-16-1108

117. Casneuf T, Xu XS, Adams HC, et al (2017) Effects of daratumumab on natural killer cells and impact on clinical outcomes in relapsed or refractory multiple myeloma. Blood Adv 1:21052114. doi: $10.1182 /$ bloodadvances.2017006866

118. Krejcik J, Casneuf T, Nijhof IS, et al (2016) Daratumumab depletes CD38+ immune regulatory cells, promotes T-cell expansion, and skews T-cell repertoire in multiple myeloma. Blood 128:384-394. doi: 10.1182/blood-2015-12-687749

119. Laubach JP, Paba Prada CE, Richardson PG, Longo DL (2017) Daratumumab, Elotuzumab, and the Development of Therapeutic Monoclonal Antibodies in Multiple Myeloma. Clin Pharmacol Ther 101:81-88. doi: 10.1002/cpt.550

120. Tai $Y-T$, Dillon M, Song W, et al (2008) Anti-CS1 humanized monoclonal antibody HuLuc63 inhibits myeloma cell adhesion and induces aritibody-dependent cellular cytotoxicity in the bone marrow mitieu. Blood 112:1329-1337. doi: 10.1182/blood-2007-08-107292

121. Collins SM, Bakan CE, Swartzel GD, et al (2013) Elotuzumab directly enhances NK cell cytotoxicity against myeloma via CS1 ligation: evidence for augmented NK cell function complementing ADCC. Cancer Immunol Immunother 62:1841-9. doi: 10.1007/s00262-013-1493-8

122. Tassi I, Colonna M (2005) The Cytotoxicity Receptor CRACC (CS-1) Recruits EAT-2 and Activates the PI3K and Phospholipase C Signaling Pathways in Human NK Cells. J Immunol 175:79968002. doi: 10.4049/jimmunol.175.12.7996

123. Cruz-Munoz ME, Dong Z, Shi X, et al (2009) Influence of CRACC, a SLAM family receptor coupled to the adaptor EAT-2, on natural killer cell function. Nat Immunol 10:297-305. doi: 10.1038/ ni.1693

124. Balasa B, Yun R, Belmar NA, et al (2015) Elotuzumab enhances natural killer cell activation and myeloma cell killing through interleukin-2 and TNF-a pathways. Cancer Immunol Immunother 64:61-73. doi: 10.1007/s00262-014-1610-3

125. Usmani SZ, Weiss BM, Plesner T, et al (2016) Clinical efficacy of daratumumab monotherapy in patients with heavily pretreated relapsed or refractory multiple myeloma. Blood 128:37-44. doi: 10.1182/blood-2016-03-705210

126. Lonial S, Dimopoulos M, Palumbo A, et al (2015) Elotuzumab Therapy for Relapsed or Refractory Multiple Myeloma. N Engl J Med 373:621-631. doi: 10.1056/NEJMoa1505654

127. Dimopoulos MA, Oriol A, Nahi H, et al (2016) Daratumumab, Lenalidomide, and Dexamethasone for Multiple Myeloma. N Engl J Med 375:1319-1331. doi: 10.1056/NEJMoa1607751 
128. Wang Y, Zhang Y, Hughes T, et al (2018) Fratricide of NK Cells in Daratumumab Therapy for Multiple Myeloma Overcome by Ex Vivo-Expanded Autologous NK Cells. Clin Cancer Res clincanres.3117.2017. doi: 10.1158/1078-0432.CCR-17-3117

129. Felices M, Lenvik TR, Davis ZB, et al (2016) Generation of BiKEs and TriKEs to Improve NK CellMediated Targeting of Tumor Cells. pp 333-346

130. Gleason MK, Ross JA, Warlick ED, et al (2014) CD16xCD33 bispecific killer cell engager (BiKE) activates NK cells against primary MDS and MDSC CD33+ targets. Blood 123:3016-3026. doi: 10.1182/blood-2013-10-533398

131. Vallera DA, Felices M, McElmurry R, et al (2016) IL15 Trispecific Killer Engagers (TriKE) Make Natural Killer Cells Specific to CD33+Targets while Also Inducing Persistence, in Vivo Expansion, and Enhanced Function. Clin Cancer Res 22:3440-3450. doi: 10.1158/1078-0432.CCR-15-2710

132. Tay SS, Carol H, Biro M (2016) TriKEs and BiKEs join CARs on the cancer immunotherapy highway. Hum Vaccines Immunother 12:2790-2796. doi: 10.1080/21645515.2016.1198455

133. Romee R, Foley $B$, Lenvik T, et al (2013) NK cell CD16 surface expression and function is regulated by a disintegrin and metalloprotease-17 (ADAM17). Blood 121:3599-3608. doi: 10.1182/ blood-2012-04-425397

134. Oboshi W, Watanabe T, Yukimasa N, et al (2016) SNPs rs4656317 and rs 2071048 located within an enhancer in FCGR3A are in strong linkage disequilibrium with rs396991 and influence NK cell-mediated ADCC by transcriptional regulation. Hum Immunol 77:997-1003. doi: 10.1016/j. humimm.2016.06.012

135. Lassauniere R, Shalekoff S, Tiemessen CT (2013) A novel FCGR3A intragenic haplotype is associated with increased FcgammaRIIIa/CD16a cell surface density and population differences. Hum Immunol 74:627-634. doi: 10.1016/j.humimm.2013.01.020

136. Koene HR, Kleijer M, Algra J, et al (1997) Fc gammaRIlla-158 V/F polymorphism influences the binding of IgG by natural killer cell Fc gammaRIlla, independently of the Fc gammaRIlla-48 L/R/H phenotype. Blood 90:1109-1114.

137. Bowles JA, Weiner GJ (2005) CD16 polymorphisms and NK activation induced by monoclonal antibody-coated target cells. J Immunol Methods 304:88-99. doi: 10.1016/j.jim.2005.06.018

138. Wu J, Edberg JC, Redecha PB, et al (1997) A novel polymorphism of FcyRllla (CD16) alters receptor function and predisposes to autoimmune disease. J Clin Invest 100:1059-1070. doi: 10.1172/JCI119616

139. Koene HR, Kleijer M, Algra J, et al (1997) Fc gammaRIlla-158V/F polymorphism influences the binding of IgG by natural killer cell Fc gammaRIlla, independently of the Fc gammaRIIla-48L/R/H phenotype. Blood 90:1109-14.

140. Congy-Jolivet N, Bolzec A, Ternant D, et al (2008) Fc gamma Rilla expression is not increased on natural killer cells expressing the Fc gamma RIlla-158V allotype. Cancer Res 68:976-80. doi: 10.1158/0008-5472.CAN-07-6523

141. Hatjiharissi E, Xu L, Santos DD, et al (2007) Increased natural killer cell expression of CD16, augmented binding and $A D C C$ activity to rituximab among individuals expressing the $\mathrm{FC}$ Rllla-158V/V and V/F polymorphism. Blood 110:2561-2564. doi: 10.1182/blood-2007-01-070656

142. Weng W-K, Levy R (2003) Two immunoglobulin $G$ fragment $C$ receptor polymorphisms independently predict response to rituximab in patients with follicular lymphoma. J Clin Oncol 21:3940-7. doi: 10.1200/JCO.2003.05.013

143. Cartron G, Dacheux L, Salles G, et al (2002) Therapeutic activity of humanized anti-CD20 monoclonal antibody and polymorphism in IgG Fc receptor FcgammaRIlla gene. Blood 99:754758. doi: 10.1182/blood.V99.3.754

144. Musolino A, Naldi N, Bortesi B, et al (2008) Immunoglobulin G fragment C receptor polymorphisms and clinical efficacy of trastuzumab-based therapy in patients with HER-2/neupositive metastatic breast cancer. J Clin Oncol 26:1789-96. doi: 10.1200/JCO.2007.14.8957 
145. Chong KT, Ho WF, Koo SH, et al (2007) Distribution of the FcgammaRIlla $176 \mathrm{~F} / \mathrm{V}$ polymorphism amongst healthy Chinese, Malays and Asian Indians in Singapore. Br J Clin Pharmacol 63:32832. doi: $10.1111 / j .1365-2125.2006 .02771 . x$

146. Bournazos S, Woof JM, Hart SP, Dransfield I (2009) Functional and clinical consequences of Fc receptor polymorphic and copy number variants. Clin Exp Immunol 157:244-254. doi: 10.1111/j.1365-2249.2009.03980.x

147. Nagelkerke SQ, Kuijpers TW (2015) Immunomodulation by IVlg and the role of Fc-gamma receptors: Classic mechanisms of action after all? Front Immunol. doi: 10.3389/fimmu.2014.00674

148. Breunis WB, Mirre E Van, Bruin M, et al (2008) Copy number variation of the activating FCGR2C gene predisposes to idiopathic thrombocytopenic purpura Copy number variation of the activating FCGR2C gene predisposes to idiopathic thrombocytopenic purpura. 111:1029-1038. doi: 10.1182/blood-2007-03-079913

149. Cooper DN (2010) Functional intronic polymorphisms: Buried treasure awaiting discovery within our genes. Hum Genomics 4:284. doi: 10.1186/1479-7364-4-5-284

150. Ip CLC, Loose M, Tyson JR, et al (2015) MinION Analysis and Reference Consortium: Phase 1 data release and analysis. F1000Research. doi: 10.12688/f1000research.7201.1

151. Lu H, Giordano F, Ning Z (2016) Oxford Nanopore MinION Sequencing and Genome Assembly. Genomics, Proteomics Bioinforma 14:265-279. doi: 10.1016/j.gpb.2016.05.004

152. Cornelis S, Gansemans Y, Deleye L, et al (2017) Forensic SNP Genotyping using Nanopore MinION Sequencing. Sci Rep 7:41759. doi: 10.1038/srep41759

153. Liu C, Xiao F, Hoisington-Lopez J, et al (2018) Accurate Typing of Human Leukocyte Antigen Class I Genes by Oxford Nanopore Sequencing. J Mol Diagnostics 1-19. doi: 10.1016/j. jmoldx.2018.02.006

154. Jennewein MF, Alter G (2017) The Immunoregulatory Roles of Antibody Glycosylation. Trends Immunol 38:358-372. doi: 10.1016/j.it.2017.02.004

155. Koehl U, Kalberer C, Spanholtz J, et al (2016) Advances in clinical NK cell studies: Donor selection, manufacturing and quality control. Oncoimmunology $5: e 1115178$. doi: $10.1080 / 2162402 X .2015 .1115178$

156. Romee R, Leong JW, Fehniger TA, et al (2014) Utilizing cytokines to function-enable human NK cells for the immunotherapy of cancer. Sci 2014:205796. doi: 10.1155/2014/205796

157. Neri D, Sondel PM (2016) Immunocytokines for cancer treatment: Past, present and future. Curr Opin Immunol 40:96-102. doi: 10.1016/j.coi.2016.03.006

158. Wagner K, Schulz P, Scholz A, et al (2008) The targeted immunocytokine L19-IL2 efficiently inhibits the growth of orthotopic pancreatic cancer. Clin Cancer Res 14:4951. doi: 10.1158/10780432.CCR-08-0157

159. Vahedi F, Nham T, Poznanski SM, et al (2017) Ex Vivo-Expanded Human NK Cells Survive and Proliferate in Humanized Mice with Autologous Human Immune Cells. Sci Rep 7:1-6. doi: 10.1038/s41598-017-12223-8

160. Denman CJ, Senyukov V V., Somanchi SS, et al (2012) Membrane-bound IL-21 promotes sustained Ex Vivo proliferation of human natural killer cells. PLoS One. doi: 10.1371/journal. pone. 0030264

161. Lapteva N, Durett AG, Sun J, et al (2012) Large-scale ex vivo expansion and characterization of natural killer cells for clinical applications. Cytotherapy 14:1131-1143. doi: 10.3109/14653249.2012.700767

162. Szmania S, Lapteva N, Garg T, et al (2015) Ex Vivo-expanded Natural Killer Cells Demonstrate Robust Proliferation In Vivo in High-risk Relapsed Multiple Myeloma Patients. J Immunother 38:24-36. doi: 10.1097/CJ.0000000000000059

163. Kobayashi T, Mattarollo SR (2017) Natural killer cell metabolism. Mol Immunol 0-1. doi: 10.1016/j. molimm.2017.11.021 
164. Hermanson DL, Kaufman DS (2015) Utilizing chimeric antigen receptors to direct natural killer cell activity. Front Immunol 6:1-6. doi: 10.3389/fimmu.2015.00195

165. Rezvani K, Rouce R, Liu E, Shpall E (2017) Engineering Natural Killer Cells for Cancer Immunotherapy. Mol Ther 25:1769-1781. doi: 10.1016/j.ymthe.2017.06.012

166. Klingemann H (2014) Are natural killer cells superior CAR drivers? Oncoimmunology. doi: 10.4161/onci.28147

167. Nguyen S, Dhedin N, Vernant JP, et al (2005) NK-cell reconstitution after haploidentical hematopoietic stem-cell transplantations: Immaturity of NK cells and inhibitory effect of NKG2A override GvL effect. Blood 105:4135-4142. doi: 10.1182/blood-2004-10-4113 
220 | Chapter 9 


\section{SUMMARY}

\section{SAMENVATTING}




\section{SUMMARY}

An increasing number of studies provides evidence that natural killer (NK) cell-based immunotherapy is an attractive strategy and a potentially successful cancer therapy. A unique feature of NK cells, as the name suggests, is that NK cells have the ability to kill cancer cell without prior sensitization, providing a faster elimination. Importantly, clinical studies have shown that injection of NK cells was safe and well tolerated. Moreover, a small number of patients with hematological malignancies achieved complete remission and an increased disease-free survival upon NK cell infusion. However, results from different studies -especially on solid tumors- showed that the efficacy of NK cell-based immunotherapy is still modest. This could be due to the low number of infused NK cells, inadequate in vivo expansion, problem with homing to tumor sites, and the suppressive tumor microenvironment (TME) (chapter 1).

In this thesis we focused on the suppressive TME and the NK-cell antitumor response. We studied and discussed the possible strategies and the feasibility to boost NK-cell tumor-killing capacity in the TME from the biology, immunology, and clinical perspective. Combination strategies to maximize NK-cell activation, reduce NK-cell inhibition, and sensitize tumor cell would be a key to unleash the NK-cell full potential.

The discrepancy between the success seen in vitro and the limited efficacy in vivo of NK cell-based immunotherapy could be due to the lack of a representative TME in vitro. In our previous study, we showed that hypoxia, a factor present in the multiple myeloma (MM) TME, inhibited the effectivity of NK-cell cytotoxicity against MM. We also showed that preactivating NK-cell with a high dose of IL-2 could restore NK-cell anti-multiple myeloma (MM) response. Since the MMTME is far more complex in vivo, we studied the effect of combination of TME factors (TMEFs) on the anti-MM response of IL-2 activated NK-cells in chapter 2. We showed that NK-cell pre-activation with IL-2 alone was not sufficient to elicit NK-cells anti-MM response when other suppressive TME factor such as lactate or prostaglandin E2 (PGE2) were present in addition to hypoxia. Using daratumumab, a monoclonal antibody against CD38, we aimed to boost NK-cell activation via antibody-dependent cell-mediated cytotoxicity (ADCC). We showed that daratumumab augmented NK-cell activation and enhanced NK-cell killing against MM cells expressing high CD38 but not against CD38 low or negative MM cells. Additionally, the absence of target cells expressing high levels of CD38 resulted in a higher number of dead NK-cells due to fratricide as NK-cells also express CD38. Since previous studies suggested that killer immunoglobulin-like receptor (KIR)ligand mismatched NK-cells were the better effector cells compared to KIR-ligand matched NK cells, we investigated whether a combination of ADCC and KIR-ligand mismatched NK cells could further improve the NK-cell anti-MM response. We observed that daratumumab 
enhanced degranulation of all NK cell subsets and that the combination of ADCC and KIRligand mismatched NK cells could further enhance NK-cell activity in the presence of TMEFs. This shows that a combination therapy of maximizing NK-cell activation by triggering ADCC and minimizing inhibitory signal through selection of KIR-ligand status could be a strategy to improve the clinical efficacy of NK cell-based immunotherapy.

In NK-cells, ADCC is mediated by CD16a receptor (FcyRIlla). It has been described that a polymorphism in the CD16a gene (FCGR3A gene) could influence the clinical outcome of monoclonal antibody therapy. However, only a few polymorphisms have been described in literature to date. In chapter 3, we studied the polymorphisms within the FCGR3A gene using the 1000 Genomes project database. We showed that the FCGR3A gene is more polymorphic than currently described. More than two third of the polymorphisms analyzed were located in the introns and only about one sixth were located in the exon regions. It would be interesting to study the functional relevance of these polymorphisms as it could provide extra information for designing an antibody and or NK cell-based immunotherapy. As there is no standard method for the detection of FCGR3A gene polymorphisms, we developed two gene-sequencing methods for full-length gene identification of FCGR3A gene polymorphisms using a Sanger-based method and nanopore MinION-based method, a novel sequencing method. Using these two methods, we were able to detect both known and new polymorphisms within the FCGR3A gene. Although further optimization and validation is required, we showed that MinION could be a more efficient method to perform a direct full-length FCGR3A gene sequencing. Using this technique, we could perform full-gene sequencing of large number of samples in a relatively short time and therefore it could be attractive for a more high throughput setting requiring a full-length sequencing.

To kill or not to kill a cancer cell, NK-cells rely on the activating and inhibitory signals received via the interaction of the activating and inhibitory receptors with their respective ligands. One strategy to fine tune the NK-cell antitumor response could be by targeting the inhibitory signalling via KIRs, NKG2A, and their ligands, the classical human leukocyte antigen (HLA) class I (KIR) and HLA-E (NKG2A). In chapter 4-6, we extensively discussed this strategy. In chapter 4, we discussed a more detailed role of HLA-E, a non-classical class I HLA molecule, in the context of stem cell transplantation and cancer. HLA-E can bind to either an activating receptor (NKG2C) or an inhibitory receptor (NKG2A), with a higher affinity to the inhibitory receptor. Current studies showed that HLA-E can be upregulated in cancer, possibly as an immune-evasion mechanism. Both membrane-bound and soluble HLA-E have been demonstrated to suppress the NK-cell antitumor response. Although several mechanisms regulating HLA-E expression have been identified, the exact roles of HLA-E in the tumor microenvironment as well as its regulation are still largely unknown. 
Further investigations would be crucial to provide a better insight on the immunoregulatory function of HLA-E and the strategies to interfere with it to improve the clinical outcome of NK cell-based immunotherapy.

One of the limitations of many of the current NK cell-based immunotherapy protocols is the relatively low number of NK cells injected to a patient. To enable infusion of higher numbers of functional NK cells, our group aims to develop ex vivo-expanded NK cells as a therapy to treat patients with cancer. However, we and other groups observed that the majority of the expanded NK cells is NKG2A positive. Given that NKG2A could potentially inhibit NK-cell upon the binding with HLA-E on target cells, we investigated the relevance of NKG2A and HLA-E interaction on the antitumor response of IL-2 activated NK cells against primary MM cells and MM cell lines in chapter $\mathbf{5}$. We observed that there were higher numbers of degranulating activated NKG2A+ NK cells against HLA-deficient K562 cells, and HLA-competent MM cell lines expressing only low levels of HLA-E, irrespective to the presence of KIRs. However, when HLA-E was overexpressed by the target cell, NKG2A+ KIR- NK-cell degranulation was inhibited. This underlines that the expression level of HLA-E on the target cell is important to elicit an inhibitory response on activated NK cells. NKG2A+ NK cells were not inhibited by the HLA-E levels present on primary MM cells obtained from patients and blocking of the receptor did not enhance degranulation of NKG2A+ subsets. Daratumumab addition enhanced NK-cell degranulation of all subsets. Moreover, KIR-NKG2A- the "unlicensed" NK cells showed a comparable response to KIR+ or NKG2A+ subsets. This demonstrates that all subsets of NK cells can contribute to tumor clearance when a potent stimulation is provided via CD16. All in all, this study shows that NKG2A receptor might not be a disadvantage for NK cell-based therapy, when NK cells are properly activated. Furthermore, the licensing effect of NKG2A might be beneficial for an antitumor response. However, NKG2A blocking might be crucial for unactivated NK cells or circulating NK cells within the tumor site or in a situation where tumor cells express high levels of HLA-E.

Although many tumor cells downregulate the classical class I HLA molecules to escape CD8+ T cells, many of these tumors are positive for HLA-E. As the majority of NK cells express NKG2A, HLA-E-expressing tumor cells could also be protected against NK cells. We observed that primary MM cells express classical class I HLA as well as HLA-E, contributing their resistance to NK-cell killing. In chapter 6, we addressed this issue and discussed the role of KIR and NKG2A in NK-cell anti-MM response as well as the strategies to maximize the clinical efficacy of allogeneic NK cell-based therapy to treat patients with MM. As the expression of both classical class I HLA and HLA-E on MM cells reduces NK-cell activation via inhibitory signaling, creating a KIR-ligand mismatched situation between NK cells and MM cells is necessary. We demonstrated in chapter $\mathbf{2}$ and 5, that indeed alloreactive KIR-ligand mismatched NK cells were the better effector cells compared to KIR-ligand matched NK 
cell against MM. Creating KIR-ligand mismatched in patients with MM can be achieved by several methods. First, creating missing-self by KIR-ligand mismatching based in genotypes in the allogeneic stem cell transplantation (SCT) setting and adoptive transfer (of ex vivoexpanded NK cells) setting. A combination of these two methods from the same donor could also be an interesting strategy as allogeneic SCT using haploidentical donors became a routine procedure in the clinic and the infusion of ex vivo-expanded NK-cell has proven to be safe. Second, using antibodies such as lirilumab (anti-KIR) or monalizumab (anti-NKG2A). This method would especially be useful in conditions such as; when creating KIR-ligand mismatch genotypically is impossible (approximately $30 \%$ of the patients), or when tumor cells express a high level of HLA-E, or when NK cells are unactivated. Third, using agents such as proteasome inhibitors (bortezomib, lactacystin, or carfilzomib) to reduce classical class I HLA expression on MM cells. To maximize the NK-cell response, a combination strategy with agents which increase NK-cell activation (such as an ADCC-triggering antibody or an antiPD1 antibody) could be crucial.

One of the biggest challenges for NK cell-based immunotherapy, as discussed in chapter $\mathbf{1}$, is the suppressive TME. This TME is not only a challenge for NK cell therapy but also contributes to resistance to $T$ cell based immunotherapy and it can contribute to drug resistance. Hypoxic conditions in the tumor site could lead to an altered metabolism of tumor cells, such as increased glucose metabolism resulting in low glucose level and high level of lactate (as a by product). Recent studies in MM cell lines showed that hypoxia-inducible factor 1 alpha (HIF1a) was upregulated by MM cells as a consequence of hypoxia, resulting in an increased glucose metabolism by MM cells. In chapter 7, we studied whether this was true for primary MM cells and whether it affects the NK-cell anti-MM response. We observed that glucose levels in the bone marrow samples of patients with MM were lower compared to the glucose levels in the bone marrow of healthy controls. This is an important finding as to date no other studies have shown this. As ex vivo-expanded NK cells are cultured in a relatively high concentration of glucose, we investigated whether the exposure of low glucose (in the TME) could compromise the NK-cell anti-MM response. We observed that a low glucose concentration during short- (overnight) or longer- term culture (4 days) did not compromise cytotoxicity or viability of highly activated NK-cells. Quite the opposite, low glucose concentration might even be favorable during culture, activation and killing process. Although further confirmation and investigation are necessary, an adaptation of the culture protocol of ex vivo-expanded NK-cells might be warranted.

Chapter 8 summarized all the important observations and findings described in this thesis. Possible challenges for NK cell-based immunotherapy and possible strategies to overcome these challenges are discussed with future perspective for the refinement of NK cell-based immunotherapy. 


\section{SAMENVATTING}

Een toenemend aantal studies levert bewijs dat natural-killer (NK)-cel gebaseerde immunotherapie een aantrekkelijke strategie en een mogelijk succesvolle kankertherapie is. Een uniek kenmerk van NK-cellen is, zoals de naam doet vermoeden, dat NK-cellen het vermogen hebben om kankercellen te doden zonder voorafgaande sensibilisatie, wat een snellere eliminatie oplevert. Belangrijk is dat klinische studies hebben aangetoond dat injectie van NK-cellen veilig was en goed werd verdragen. Bovendien bereikte een klein aantal patiënten met hematologische maligniteiten volledige remissie en een verhoogde ziektevrije overleving na NK-cel-infusie. Resultaten van verschillende onderzoeken, met name op solide tumoren, toonden echter aan dat de werkzaamheid van op NK-cel-gebaseerde immunotherapie nog steeds bescheiden is. Dit kan te wijten zijn aan het lage aantal geïnfuseerde NKcellen, onvoldoende in vivo-expansie, probleem met transport naar de tumor en de suppressieve tumor-micro-omgeving (TMO) (hoofdstuk 1).

In dit proefschrift hebben we ons gericht op de suppressieve TMO en de NK-cel antitumorrespons. We bestudeerden en bediscussieerden de mogelijke strategieën en de haalbaarheid om NK-cel tumor-doding capaciteit in de TMO te verhogen vanuit biologisch, immunologisch en klinisch perspectief. Combinatiestrategieën om NK-celactivatie te maximaliseren, NK-celremming te verminderen en tumorcellen gevoelig te maken, zouden een sleutel kunnen zijn om het NK-celpotentieel volledig te ontketenen.

De discrepantie tussen het succes in vitro en de beperkte werkzaamheid in vivo van op NK-cel-gebaseerde immunotherapie kan te wijten zijn aan het ontbreken van een representatief TMO in vitro. In onze vorige studie toonden we aan dat hypoxie, een factor die aanwezig is in de multipel myeloom (MM) TMO, de werkzaamheid van NK-cel cytotoxiciteit tegen MM remde. We toonden ook aan dat het pre-activeren van NKcellen met een hoge dosis IL-2 de NK-cel, de anti-multipel myeloom (MM) -respons kunnen herstellen. Omdat de MM TMO in vivo veel complexer is, hebben we het effect bestudeerd van de combinatie van TMO-factoren (TMOF's) op de anti-MM-respons van IL-2 geactiveerde NK-cellen in hoofdstuk 2. We hebben aangetoond dat NK-cel preactivatie met alleen IL-2 niet voldoende was om in NK-cellen een anti-MM-reactie op te wekken wanneer andere onderdrukkende TMO-factoren zoals lactaat of prostaglandine E2 (PGE2) aanwezig waren naast hypoxie. Met behulp van daratumumab, een monoklonaal antilichaam tegen CD38, wilden we de activatie van NK-cellen stimuleren via antilichaamafhankelijke celgemedieerde cytotoxiciteit (ADCC). We toonden aan dat daratumumab de NK-cel activatie verhoogde en NK-celdoding tegen MM-cellen 
met hoge CD38 expressie verbeterde. Dit was niet het geval voor MM-cellen met lage of negatieve CD38 expressie. Bovendien resulteerde de afwezigheid van MMcellen met hoge expressie van CD38 in een hoger aantal dode NK-cellen als gevolg van fraticide aangezien NK-cellen zelf ook CD38 tot expressie brengen. Omdat eerdere studies suggereerden dat killer immunoglobuline-achtige receptor (KIR) -ligand mis-gematchte NK-cellen betere effectorcellen waren in vergelijking met KIR-ligandgematchte NK-cellen, onderzochten we of een combinatie van ADCC en KIR-ligand mis-gematchte NK-cellen verder verder konden bijdragen aan de NK-cel anti-MM respons. We hebben waargenomen dat daratumumab de degranulatie van alle NKcelsubsets verhoogde en dat de combinatie van ADCC en KIR-ligand mis-gematchte NK-cellen de NK-celactiviteit verder kon verbeteren in de aanwezigheid van TMOF's. Dit toont aan dat een combinatietherapie van het maximaliseren van NK-celactivering door triggeren van ADCC en het minimaliseren van remmend signaal door selectie van KIR-ligandstatus een strategie zou kunnen zijn om de klinische werkzaamheid van op NK-cel-gebaseerde immunotherapie te verbeteren.

In NK-cellen wordt ADCC gemedieerd door de CD16a-receptor (Fc囚RIIIa). Er is beschreven dat een polymorfisme in het CD16a-gen (FCGR3A-gen) de klinische uitkomst van monoklonale antilichaamtherapie zou kunnen beïnvloeden. In de literatuur zijn echter tot op heden slechts enkele polymorfismen beschreven. In hoofdstuk $\mathbf{3}$ hebben we de polymorfismen binnen het FCGR3A-gen bestudeerd met behulp van de 1000 Genomes projectdatabase. We hebben aangetoond dat het FCGR3A-gen meer polymorf is dan momenteel wordt beschreven. Meer dan tweederde van de geanalyseerde polymorfismen bevonden zich in de introns en slechts ongeveer een zesde bevond zich in de exon-regio's. Het zou interessant zijn om de functionele relevantie van deze polymorfismen te bestuderen omdat het extra informatie zou kunnen verschaffen voor het ontwerpen van een antilichaam en of NK-cel-gebaseerde immunotherapie. Omdat er geen standaardmethode is voor de detectie van FCGR3A-genpolymorfismen, hebben we twee genssequentiemethoden ontwikkeld voor gen-identificatie van de volledige lengte van FCGR3A-genpolymorfismen met behulp van een op Sanger gebaseerde methode en een op nanometer MinION-gebaseerde methode, een nieuwe sequentiemethode. Met behulp van deze twee methoden konden we zowel bekende als nieuwe polymorfismen binnen het FCGR3A-gen detecteren. Hoewel verdere optimalisatie en validatie vereist is, hebben we aangetoond dat MinION een efficiëntere methode kan zijn om een directe FCGR3A-gensequentie van volledige lengte uit te voeren. Met behulp van deze techniek konden we in een relatief korte tijd de volledige gensequentie in kaart brengen van een groot aantal monsters en daarom zou het aantrekkelijk kunnen zijn waar een sequentiebepaling van een volledige gen vereist met een meer hogere verwerkingscapaciteit. 
Om een kankercel al dan niet te doden, vertrouwen NK-cellen op de activerende en remmende signalen die worden ontvangen via de interactie van de activerende en remmende receptoren met hun respectievelijke liganden. Eén strategie om de NK-cel antitumorrespons te verfijnen zou kunnen zijn door te richten op de remmende signalering via KIR's, NKG2A en hun liganden, het klassieke menselijke leukocytantigeen (HLA) klasse I (KIR) en HLA-E (NKG2A). In hoofdstuk 4-6 hebben we deze strategie uitvoerig besproken. In hoofdstuk 4 bespraken we een meer gedetailleerde rol van HLA-E, een niet-klassieke klasse I HLA-molecule, in de context van stamceltransplantatie en kanker. HLA-E kan binden aan ofwel een activerende receptor (NKG2C) of een remmende receptor (NKG2A), met een hogere affiniteit voor de remmende receptor. Recente onderzoeken hebben aangetoond dat HLA-E kan worden opgereguleerd bij kanker, mogelijk als een mechanisme voor het ontwijken van de immuunrespons. Van zowel membraangebonden als oplosbare HLA-E is aangetoond dat ze de NK-cel antitumorreactie onderdrukken. Hoewel er verschillende mechanismen zijn geïdentificeerd die HLA-E-expressie reguleren, zijn de precieze rollen van HLA-E in de micro-omgeving van de tumor en de regulatie ervan nog grotendeels onbekend. Verder onderzoek zou cruciaal zijn om een beter inzicht te krijgen in de immunoregulerende functie van HLA-E en de strategieën om deze te verstoren om de klinische uitkomst van op NK-cel-gebaseerde immunotherapie te verbeteren.

Een van de beperkingen van veel van de huidige op NK-cel-gebaseerde immunotherapieprotocollen is het relatief lage aantal NK-cellen dat bij de patiënt is geïnjecteerd. Om infusie van hogere aantallen functionele NK-cellen mogelijk te maken, wil onze groep ex vivo methode ontwikkelen om NK-cellen te vermeerderen als een therapie voor de behandeling van patiënten met kanker. Wij en andere groepen zagen echter dat de meerderheid van de geëxpandeerde NK-cellen NKG2Apositief is. Gegeven dat NKG2A mogelijk NK-cellen remt op de binding met HLA-E aan MM-cellen, onderzochten we de relevantie van NKG2A en HLA-E interactie op de antitumorrespons van IL-2 geactiveerde NK-cellen tegen primaire MM-cellen en MM-cellijnen in hoofdstuk 5. We hebben waargenomen dat er hogere aantallen degranulerende geactiveerde NKG2A+ NK-cellen waren tegen HLA-deficiënte K562cellen en HLA-competente MM-cellijnen die alleen lage HLA-E-niveaus tot expressie brengen, ongeacht de aanwezigheid van KIR's. Toen HLA-E echter tot overexpressie werd gebracht door de doelwit-cel, werd NKG2A+ KIR-NK-celdegranulatie geremd. Dit onderstreept dat het expressieniveau van HLA-E op de doelwitcel belangrijk is om een remmende respons op geactiveerde NK-cellen op te wekken. NKG2A+ NK-cellen werden niet geremd door de HLA-E-niveaus die aanwezig waren op primaire MMcellen die waren verkregen van patiënten en blokkering van de receptor versterkte 
de degranulatie van NKG2A+ -subsets niet. Daratumumab toevoeging verbeterde NK-cel degranulatie van alle subsets. Bovendien vertoonden KIR-NKG2A- de "nietgelicentieerde" NK-cellen een vergelijkbare reactie op KIR+ of NKG2A+ -subsets. Dit toont aan dat alle subsets van NK-cellen kunnen bijdragen aan tumorklaring wanneer een krachtige stimulering via CD16 wordt verschaft. Al met al laat deze studie zien dat NKG2A-receptor misschien geen nadeel is voor een op NK-cel-gebaseerde therapie, wanneer NK-cellen correct worden geactiveerd. Bovendien kan het licentieeffect van NKG2A gunstig zijn voor een antitumorreactie. Echter, NKG2A-blokkering kan cruciaal zijn voor ongeactiveerde NK-cellen of circulerende NK-cellen binnen de tumoromgeving of in een situatie waarin tumorcellen hoge niveaus van HLA-E tot expressie brengen.

Hoewel veel tumorcellen de klassieke klasse I HLA-moleculen downreguleren om te ontsnappen aan CD8+ T-cellen, zijn veel van deze tumoren positief voor HLA-E. Aangezien de meeste NK-cellen NKG2A tot expressie brengen, zouden HLA-E tot expressie brengende tumorcellen ook tegen NK-cellen kunnen worden beschermd. We hebben waargenomen dat primaire MM-cellen klassieke klasse I-HLA alsook HLA-E tot expressie brengen, hetgeen hun resistentie tegen NK-celdoding bijdraagt. In hoofdstuk 6 hebben we dit onderwerp besproken en de rol van KIR en NKG2A in NK-cel anti-MM-respons besproken, evenals de strategieën om de klinische werkzaamheid van allogene NK-celgebaseerde therapie voor de behandeling van patiënten met MM te maximaliseren. Omdat de expressie van zowel klassieke klasse I HLA als HLA-E op MM-cellen NK-celactivering via remmende signalering vermindert, is het creëren van een KIR-ligand mis-gematchte situatie tussen NK-cellen en MM-cellen noodzakelijk. We toonden in hoofdstuk $\mathbf{2}$ en $\mathbf{5}$ aan dat inderdaad alle reactieve KIRligand mis-gematchte NK-cellen de betere effectorcellen waren in vergelijking met KIR-ligand gematchte NK-cellen tegen MM. Het creëren van KIR-ligand mis-gematcht bij patiënten met MM kan op verschillende manieren worden bereikt. Ten eerste, het creëren van missing-self door KIR-ligand mismatching op basis van genotyperen in de allogene stamceltransplantatie (SCT) setting en adoptieve transfer (van ex vivo vermeerderde NK-cellen) setting. Een combinatie van deze twee methoden van dezelfde donor zou ook een interessante strategie kunnen zijn, aangezien allogene SCT met behulp van haplo-identieke donoren een routineprocedure werd in de kliniek en de infusie van ex vivo vermeerderde NK-cellen veilig is gebleken. Ten tweede, met behulp van antilichamen zoals lirilumab (anti-KIR) of monalizumab (antiNKG2A). Deze methode zou vooral handig zijn in omstandigheden zoals; wanneer het creëren van een KIR-ligand mismatch genotypisch onmogelijk is (ongeveer $30 \%$ van de patiënten), of wanneer tumorcellen een hoog niveau van HLA-E tot expressie brengen, of wanneer NK-cellen niet-geactiveerd zijn. Ten derde, met behulp van 
middelen zoals proteasoomremmers (bortezomib, lactacystin of carfilzomib) om klassieke klasse I HLA-expressie op MM-cellen te verminderen. Om de NK-celrespons te maximaliseren, zou een combinatiestrategie met middelen die NK-celactivering verhogen (zoals een ADCC-activerend antilichaam of een anti-PD1-antilichaam) cruciaal kunnen zijn.

Een van de grootste uitdagingen voor NK-cel-gebaseerde immunotherapie, zoals besproken in hoofdstuk 1, is de onderdrukkende TMO. Deze TMO is niet alleen een uitdaging voor NK-celtherapie, maar draagt ook bij aan resistentie bij T-celgebaseerde immunotherapie en kan bijdragen aan resistentie tegen geneesmiddelen. Hypoxische aandoeningen in de tumoromgeveing kunnen leiden tot een veranderd metabolisme van tumorcellen, zoals een verhoogd glucosemetabolisme, resulterend in een laag glucosegehalte en een hoog lactaatniveau (als bijproduct). Recente studies in MM-cellijnen toonden aan dat hypoxie-induceerbare factor 1 alfa (HIF1a) door MM-cellen werd opgereguleerd als gevolg van hypoxie, resulterend in een verhoogd glucosemetabolisme door MM-cellen. In hoofdstuk 7 hebben we onderzocht of dit waar was voor primaire MM-cellen en of dit de NK-cel anti-MM-respons beïnvloedt. We hebben vastgesteld dat de glucosespiegels in de beenmergmonsters van patiënten met MM lager waren in vergelijking met de glucosespiegels in het beenmerg van gezonde controles. Dit is een belangrijke bevinding omdat tot op vandaag de dag geen andere onderzoeken dit hebben aangetoond. Omdat ex vivo-geëxpandeerde NK-cellen worden gekweekt in een relatief hoge concentratie glucose, onderzochten we of de blootstelling van lage glucose (in de TMO) de NK-cel anti-MM-respons kon aantasten. We hebben waargenomen dat een lage glucoseconcentratie tijdens korte- (gedurende de nacht) of langere termijn (4 dagen) de cytotoxiciteit of levensvatbaarheid van sterk geactiveerde NK-cellen niet in gevaar bracht. Integendeel, een lage glucoseconcentratie kan zelfs gunstig zijn tijdens het kweek-, activerings- en dodingproces. Hoewel verdere bevestiging en onderzoek nodig zijn, kan een aanpassing van het kweekprotocol van ex vivo-geëxpandeerde NK-cellen gerechtvaardigd zijn.

Hoofdstuk 8 vat alle belangrijke observaties en bevindingen samen die in dit proefschrift worden beschreven. Mogelijke uitdagingen voor NK-cel-gebaseerde immunotherapie en mogelijke strategieën om deze uitdagingen te overwinnen, worden besproken met toekomstperspectief voor de verfijning van op NK-celgebaseerde immunotherapie. 
Samenvatting | 231

$\frac{a}{\frac{1}{d}}$ 
232 Chapter 10 


\section{VALORIZATION}

\section{LIST OF PUBLICATIONS}

\section{CURRICULUM VITAE}

ACKNOWLEDGEMENT 


\section{VALORIZATION}

The 2018 GLOBOCAN data estimated that there would be around 18.1 million new cancer cases and 9.6 million cancer deaths worldwide in 2018. Although several types of cancer have a better chance of remission than other types after surgery/ chemo-/radiotherapy, cancer is mostly still an incurable disease. Multiple myeloma (MM), a plasma cell malignancy, is an example of a malignancy with a low survivalrate, regardless of an increased progression-free survival in the last few years due to the discovery of new drugs besides the conventional chemotherapy. NK cell-based immunotherapy might be a promising alternative therapy for MM patients as recent studies showed that NK cells in MM patients are often dysfunctional. In addition, recent clinical trials in MM patients using either autologous or allogeneic ex vivoexpanded NK cells showed that it is safe and feasible. However, the clinical efficacy reported was still limited.

One of the possible limitations of the clinical efficacy of an NK cell-based immunotherapy could be the under representation of the tumor microenvironment in an in vitro testing. In this thesis (chapter 2 and 5) we showed that the addition of selected factors (hypoxia, lactate, PGE2) could negatively affect NK-cell killing against MM cells. In another study in this thesis (chapter 7), another factor (low glucose) on the other hand, seemed to be beneficial for NK cells. Given these results, a development of a more representative in vitro model of tumor microenvironment resembling in vivo (patient's) tumor microenvironment would be pivotal to better predict the effect of the treatment in vivo. This could reduce unnecessary (further) developments of treatment which are unlikely to give a desirable effect in patients.

To be able to mediate an antitumor response in the tumor microenvironment, NK cells need to be properly activated. We showed in this thesis that a combination strategy of KIR-ligand mismatched NK cells with a monoclonal antibody resulted in a better killing of tumor cells in the presence of selected biochemical factors mimicking the tumor microenvironment. At the same time, more importantly, we showed that the administration of an antibody had two less desirable outcomes, 1) the expression of the target antigen had to be high on the target cells otherwise the addition of the monoclonal antibody did not enhance NK cells killing against tumor cells, 2) when the target antigen is also expressed on NK cells, using the monoclonal antibody could be detrimental for NK cells as a result of fratricide (NK-NK killing). To overcome these unnecessary "side effects", several strategies could be considered. First, proper timing between the antibody administration and the injection of NK-cell adoptive transfer. Second, pretreatment of NK-cells with a $F(a b)_{2}$ fragment of the antibody 
prior to injection. Third, development of a bi-specific or tri-specific antibody to better target tumor cells. Fourth, development of an antibody which could still trigger an antibody-dependent cell-mediated cytotoxicity (ADCC) on NK cell despite the low/ intermediate level of antigen expressed on the target cells. A proper design of an antibody is highly crucial for the success of a combination therapy with an NK cellbased immunotherapy.

In this thesis, we also described that a factor that potentially contributes to the success of antibody therapy is the CD16a (FcyRIIla) receptor. A few polymorphisms of the FCGR3A gene, the gene encoding the CD16a receptor, have been described to influence the binding of CD16a to an antibody. We are the first to provide an extensive overview of FCGR3A gene polymorphisms using the 1000 Genome project database. Additionally, we also successfully developed two gene-sequencing methods to detect a full-length FCGR3A gene polymorphisms: a Sanger based and a MinION based, a novel sequencing method. From this study, both the overview and the sequencing methods together could be used to: 1) further investigate the functional relevance of FCGR3A gene polymorphisms on NK-cell ADCC capacity, 2) design an antibody or CAR-NK cells, 3) select an NK-cell donor. A major advantage of the nanopore MinION strategy would be that it enables faster analysis of full length gene polymorphism. Due to the possibility to barcode individual samples or genes, it also enables the simultaneous analysis of samples which may reduce the costs of this technology.

Another key limiting factor for an NK cell-based immunotherapy is to obtain sufficient numbers of functional NK cells for the infusion to a cancer patient. Although there is no consensus for the minimum or maximum number of NK-cells that can or should be injected to a patient, current clinical trials have gone up to $10^{8} \mathrm{NK}$ cells $/ \mathrm{kg}$. An advantage of donor NK cell-based immunotherapy is that donor NK cells could be ex vivo-expanded as an off-the-shelf product and hence a readily available product. As NK cells do not attack healthy cells infusion of allogeneic NK cell is safe and KIR-ligand mismatched NK cells seemed to be the better effector cells. Therefore, a universal "perfect" NK-cell donor could be selected based on the genotype; that is when donor NK cells express all licensed KIRs. Our group has been working for years to develop an optimized protocol and technology to expand NK cells ex vivo aiming to produce $10^{10} \mathrm{NK}$ cells. At CiMaas, a spin-off company founded by Prof. dr. G. M. J. Bos and Dr. W. T. V. Germeraard, it is now possible to manufacture a GMP-grade ex vivo-expanded NK-cell product. 
One of the very promising strategies to use those expanded NK cells is by combining them with a haploidentical stem cell transplantation. Our group is currently leading the first Phasel/II multicenter study where MM patients receive a haploidentical stem cell transplantation and donors are selected by the Transplantation Immunology lab, based on the presence of a KIR-ligand mismatch. Although the study has not been completed yet, it demonstrated the feasibility and safety of the approach. From the study, we also learned that it takes 30-60 days before mature NK cells are circulating in the patient. To further enhance clinical responses, haplo-SCT could be combined with infusion of NK cells form the same donor expanded according to the CiMaas protocol. The expanded NK cells could potently mediate their anti-tumor responses in the first two months while persistence of the response will result from donor NK cells that developed from the stem cell graft. This procedure has been shown to be very efficient by our close collaborators at Cytosen and MD Anderson. Based on the results described in this thesis, $A D C C$ or blocking antibodies could be combined with haplo-SCT and NK infusion to further potentiate the response. A further development for these ex vivo-expanded NK cells would include molecular modifications of the receptors (termed chimeric antigen receptor or often abbreviated CAR) to better target tumor cells. As an off-the-shelf product, ex vivo-expanded NK cells would be stored in the freezer for storage. Previous studies, however, have reported that NK cell viability decreased after a freeze thawing procedure. Therefore, a further technology optimization/development on the freeze-thawing protocol/technology might be necessary and interesting aspect to develop.

In summary, data presented in this thesis serve as a confirmation and follow up of our previous studies as well as a starting point and foundation of our subsequent studies. Altogether, the findings described in this thesis contribute to the refinement of an NK cell-based immunotherapy which may contribute to novel treatment options for MM patients that provide curative responses and preferably have a low level of toxicity. 


\title{
LIST OF PUBLICATIONS
}

\author{
PUBLICATIONS (IN THIS THESIS)
}

Mahaweni NM, Ehlers FAl, Bos GMJ, Wieten L. Tuning NK cell anti-myeloma reactivity by targeting inhibitory signaling via KIR and NKG2A. Under review at Frontiers in Immunology. 2018

Mahaweni NM, Olieslagers TI, Rivas IO, Molenbroeck SJJ, Groeneweg M, Bos GMJ, Tilanus MGJ, Voorter CEM, and Wieten L. A comprehensive overview of FCGR3A gene variability by full-length gene sequencing including the identification of V158F polymorphism. Sci Rep. 2018 Oct; 8:15983. doi: $10.1038 / s 41598-018-34258-1$

Mahaweni NM, Ehlers FAI, Sarkar S, Janssen JWH, Tilanus MGJ, Bos GMJ and Wieten L. "NKG2A expression is not per se detrimental for the anti-multiple myeloma activity of activated natural killer cells in an in vitro system mimicking the tumor microenvironment". Front. Immunol. 2018 Jun. 9:1415. doi: 10.3389/fimmu.2018.01415

Mahaweni NM, Bos GMJ, Mitsiades CS, Tilanus MGJ, Wietens L.“'Daratumumab augments alloreactive natural killer cell cytotoxicity towards CD38+ multiple myeloma cell lines in a biochemical context mimicking tumour microenvironment conditions." Cancer Immunol Immunother. 2018 Jun;67(6):861-872. doi: 10.1007/s00262-018-2140-1. Epub 2018 Mar 2.

Wieten L, Mahaweni NM, Voorter CE, Bos GM, Tilanus MG. "Clinical and immunological significance of HLA-E in stem cell transplantation and cancer." Tissue Antigens. 2014 Dec;84(6):523-35. doi: 10.1111/tan.12478. Review.

PUBLICATIONS (NOT IN THIS THESIS)

Aerts JGJV, de Goeje PL, Cornelissen R, Kaijen-Lambers MEH, Bezemer K, van der Leest $\mathrm{CH}$, Mahaweni NM, Kunert A, Eskens FALM, Waasdorp C, Braakman E, van der Holt B, Vulto AG, Hendriks RW, Hegmans JPJJ, Hoogsteden HC. "Autologous Dendritic Cells Pulsed with Allogeneic Tumor Cell Lysate in Mesothelioma: From Mouse to Human." Clin Cancer Res. 2018 Feb 15;24(4):766776. doi: 10.1158/1078-0432.CCR-17-2522. Epub 2017 Dec 12.

Mahaweni NM, Kaijen-Lambers ME, Dekkers J, Aerts JG, Hegmans JP. “Tumour-derived exosomes as antigen delivery carriers in dendritic cell-based immunotherapy for malignant mesothelioma." J Extracell Vesicles. 2013 Oct 24;2. doi: 10.3402/jev.v2i0.22492. eCollection 2013. 


\section{CURRICULUM VITAE}

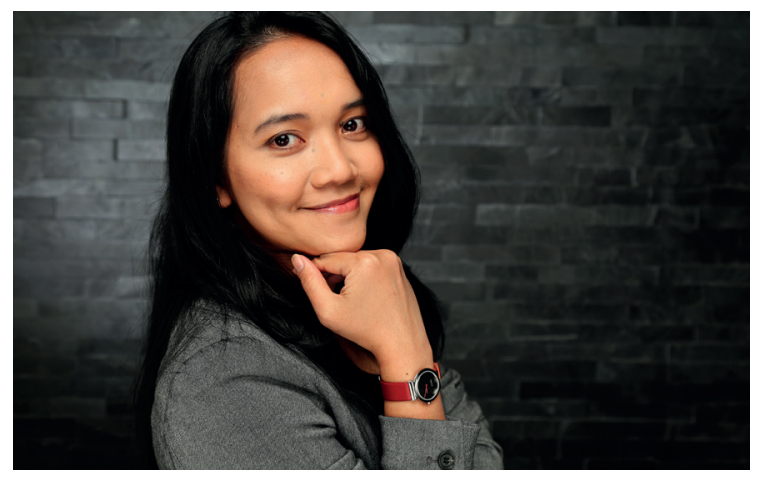

Niken Miranti Mahaweni was born on 20 November 1986, in Jakarta, Indonesia. She grew up in different cities in Indonesia and has lived in more than 10 different houses. She finished her elementary and junior secondary school in Lampung (Sumatera island). Upon completion of her junior secondary school, she discussed with her father that she would like to study in Universitas Gadjah Mada, located in Yogyakarta (Java island), to become a career diplomat. She proposed to move to Yogyakarta to do her senior secondary school in Yogyakarta as a preparation to study in Gadjah Mada. However, after an inspirational conversation with her mother, she decided to study medicine and become a doctor. In 2004, she was enrolled in the international program of medicine at Universitas Gadjah Mada.

Upon finishing her medical study and receiving her medical license in 2010, she worked at the department of internal medicine, division of hematology and medical oncology of Universitas Gadjah Mada, as a research assistant/study coordinator for clinical trials. During her work at this department, she developed her interest in biomedical research, which she viewed as an indispensable part of patient care. This motivated her to first pursue scientific training before specializing further in medicine. In 2011, she went to the Netherlands to follow molecular medicine research master program at Erasmus University Rotterdam. During her internships at the department of pulmonary medicine laboratory at Erasmus Medical Center, she discovered her research passion: immunology, especially cancer immunology/immunotherapy. Her enthusiasm rewarded her a first author paper and a student grant from the Royal Netherlands Academy of Arts and Sciences (KNAW) for her second-year research project. After completion of her master study, she got the opportunity to do her PhD training at the department of internal medicine, division of hematology, and the department of transplantation immunology/tissue typing laboratory, at Maastricht University Medical Center+ under the supervision of Prof. dr. Gerard M. J. Bos, Prof. dr. Marcel G. J. Tilanus, and Dr. Lotte Wieten. This dissertation describes the results acquired during this $\mathrm{PhD}$ training. At the moment, she is following the process to obtain a medical license in the Netherlands to continue her medical training. 


\section{AWARDS AND GRANTS}

Erasmus MC - Fellowship

Awarded by Erasmus MC University Medical Center Rotterdam - The

Netherlands to cover the tuition fees.

Academy Assistantship

Student grant awarded by KNAW (The Royal Netherlands Academy of

2012

Arts and Science)

Best Abstract - Winner

$30^{\text {th }}$ European Immunogenetics and Histocompatibility Conference in

Kos, Greece

Presentation title: "The two to tango: Antibody-dependent cellular

cytotoxicity enhances superiority of alloreactive NK cells against multiple myeloma"

Travel Grant

To attend the $16^{\text {th }}$ Annual Meeting of the Society of Natural Immunity (NK2016)

Awarded by EFIS (European Federation of Immunological Societies) and EJI (European Journal of Immunology)

GROW Science Day Poster Award 2016 - Winner

GROW School for Oncology and Developmental Biology

Faculty of Health, Medicine, and Life Sciences, Maastricht University

Best Abstract Award - Runner Up

31st European Immunogenetics and Histocompatibility Conference in Mannheim, Germany

Presentation title: "Clinically approved monoclonal antibodies and 2017 pm21 particle stimulated ex vivo-expanded alloreactive natural killer cells: a potent combination against cancer cells"

\section{ORGANIZATION AND ACTIVITIES}

CIMSA (Center for Indonesian Medical Students Activities)

Web developer for Standing Committee on Professional Exchange

$2005-2008$

SURE (Student Union Research Masters-Erasmus)

Newsletter Commission

$2012-2013$

Erasmus MC University Medical Center Rotterdam - The Netherlands

PhD representative

FHML - GROW School for Oncology and Developmental Biology 


\section{ACKNOWLEDGEMENT}

Looking back to my $\mathrm{PhD}$ journey, I thought 4 years was quite some time, but boy I was wrong.. Time flies. But like Einstein said "time flies when you are having fun". And that's true, I had fun doing and during my training $\odot$. But, as the book is done, that means my PhD training is coming to an end. And of course although this is my journey, it won't be possible to do this alone. In these last few pages, I would like to express my gratitude to all the people who have helped, supported and inspired me throughout these years. Thank you very much for making it possible!

First of all, I would like to thank my promotion team who gave me the opportunity to do my PhD training at their departments under their supervision: Prof. dr. Gerard Bos, Prof. dr. Marcel Tilanus, and Dr. Lotte Wieten. (-) Lotte, thank you very much for all the trust that you put in me. From the very beginning, you gave me the freedom to design, plan, and execute my project(s). During our discussions, you always stimulated my critical and creative thinking. I really appreciate all the feedback, inputs, and ideas you shared with me during our discussions. I loved it that I would leave your room with a long list of ideas for the project. Too bad, so much to do yet so little time. I did learn a lot from you about how to process a result (data) and writing it into a paper. You always had a clear idea how to put the data together in a paper. When we were writing an article, I was always happy when I read your e-mail "Hey Niken! Nice work!" - but then I opened the file and found almost everything was red! Haha.. Thank you so much for your help, support, patience (when I had to rehearse 1000 times for EFIs), dedication, motivations, enthusiasm, guidance and time (plus extra time when you had to look into my draft(s) during the weekend/holiday). Thank you also for giving me the chance to develop myself personally (supervising students, tutorial). It was a pleasure to work with you. I wish you a lot of success in the future with your (scientific) career.:- Gerard, thank you for your critical look and challenging view on the project. I truly appreciate it. You always reminded us what is important in the project to stay focus and not distracted from the main question. Thank you for making me learning statistics again. Thank you for your guidance, support, and constructive feedback. I wish you a lot of success with the CiMaas.

(-) Marcel, I still remember when I did my "sollicitatie-gesprek" via Skype with you, you challenged me with a question and I disagreed with your statement. After we hung up the Skype, I was honestly terrified "Oh my God, I just disagreed with the professor". But later I heard that you appreciated that I had my own opinion and reasoned it. Thank you for giving me the opportunity to work at your department. Thank you for your support and encouragement especially for the presentations at the EFIs. 
The dissertation assessment committee: Prof. dr. Dirk De Ruysscher, Prof. dr. Vivianne C. G. Tjan-Heijnen, Dr. Kasper Rouschop, Prof. dr. Irma Joosten, and Prof. dr. Tuna Mutis, thank you very much for reading, judging, and providing a positive evaluation of my dissertation.

GROW school: Prof. dr. Frans Ramaekers and Dr. Theo de Kok, thank you for your periodic supervision on my PhD traject. Thank you for your time and constructive feedback. Brigitte \& Christel, thank you so much for your help, especially with the administration and the GROW representatives matters.

I would also like to thank all the people who have willingly donated their blood for my research. Without their kind participation, the researches done in this thesis would not be possible.

A big thank you to everyone in the past and present with whom I shared the lab at the hematology division. It was like my second home to me.-) Birgit, jij bent de moeder van het lab! Jij bent streng (moet ook zo zijn, om de goede organisatie van het lab te behouden) maar tegelijkertijd ben je ontzettend begripvol en lief. Heel erg bedankt voor alles! Je hebt mij veel geholpen en gesteund afgelopen 4 jaar. Je stond altijd klaar als ik hulp nodig had (ook bij rare vraagjes/verzoekjes - kapotte apparaten, bestellingen van rare producten etc). Ik heb ook van onze chitchat genoten. Dank je wel dat je mij hebt aangemoedigd om Nederlands te praten en voor je persoonlijke wijsheid.:-) Wilfred, thank you for introducing me to the world of supervising and teaching and for your input on my project during lab meetings. O yes, and thank you for giving me the idea how nice an Audi is when you took Ying and me to the NVVI. Haha lk wens je veel succes met je bedrijf en je carrière! $\odot$ Silvie, I enjoyed working together with you. Your precision and detail working-method is exemplary. You were always such a lively person! It was always nice to have you around. Ik wens je heel veel geluk met je gezinnetje en je toekomstige baan!- Marijn, thank you for your help, especially with measuring the marrow/blood samples, and sometimes with the cell culture/isolation. It was a pleasure to have you as a roomie and as a colleague. Keep up the excellent work! $)$ Melanie, thank you for your help with a couple experiments when I was short-handed and also filling empty boxes of pipette-tips that I left especially on Friday afternoon. Monday morning, with a big smile on your face, all those boxes were already filled when I just entered the lab (-) Michel, thank you for your help and your input on my project(s) and for the nice chit-chats. Thank you for introducing me to people for collaboration. Your quick-witted remarks during meetings have helped me to fine tune my experiments $(-)$ Janine, your enthusiasm, dedication, and passion for research is an inspiration for me. Thank you for your advices (-) Gwendolyn, I wish you all the best with your PhD project $\odot$ Roel, it was a nice 
surprise to see you again in Maastricht, after Rotterdam! You are one of --the smartest but still the kindest- persons I've ever known. Thank you for your big help (also for giving me a shelter when I was homeless haha). I enjoy our scientific discussions and of course our coffee-break talks. Talking to you always gave me motivation, inspiration, and extra strength that "I can do it". Thank you for your advices and encouragement $:-$ Subhashis, thank you for your help in writing the NKG2A paper. (-) Mirelle, thank you for sharing your PhD experience when I was still a newbie, I wish you all the best with your future profession as a clinical chemist. (-) Thomas, your cool illustrations on presentations, posters or articles gave me an extra motivation to make ones as well. Thank you for the tips and tricks! (-) Tammy, professionally, I enjoyed working with you. Your hard work and hundreds plates inspired my own experiments. Haha.. Thank you because you always had the time to help me, troubleshooting in the lab, and to discuss data. Personally, you are such a sweet and kind-hearted person. I always had a good time talking to you. Thank you for the gezelligheid and friendship. (-) Ying, you're such an intelligent guy and a gentleman! Keep up the hard work, but don't forget to also have some fun. All the best for your PhD! :- Femke, I wish we started the PhD at the same time so that we could go to many courses/congresses together. It was nice to have you around. Thank you for your help with the experiments, for the discussions/brainstorming time, as well as the girly talks. Also thank you for being my fairy lab mother -always refilling my snoepjes vooraadand being my paranymph. Thank you for the friendship! I wish you all the best with your last 2 years of PhD. You'll do great! (-) Esther Houben, dank je wel voor je hulp vanuit het secretariaat.

Of course also many thanks to everyone at the department of transplantation immunology, especially (-) Christien, Mathijs, thank you for your scientific input in my projects, especially in the CD16a project. Piet (Timothy) and I will definitely miss the volleyball game with you and the team. (-) Stefan, thank you for processing the samples for the CD16a project. (-) Ben, thank you for checking the English of my articles as well as filtering the CD16a samples in the MinION. Good luck with your PhD! (-) Denise, EFI was leuker met je erbij! Ik vind jouw enthousiasme \& vrolijkheid geweldig! Succes met het afronden van je PhD. :-) Burcu, ik vond het leuk dat er ook iemand anders was die Nederlands aan het leren was. Veel succes ermee en ook met je carrière. (-) Lisette $\boldsymbol{\&}$ Veerle, dank jullie wel voor jullie hulp, vooral met het verwerken van mijn monsters. (-) Annette \& Jeroen, dank jullie wel voor jullie hulp met het afnemen van bloed. Wat een vampire was ik toch. (-) Sandra, dank je wel voor je hulp vooral met de administratie. (-)Diana, Audrey, en Brigitte, heel erg bedankt voor jullie secretariële ondersteuning! Ik vond het ook altijd leuk om even met jullie te kletsen. And of course $:-$ Timo! Thank you soooo much for your help and support during my $\mathrm{PhD}$ ! It was always nice to work with you. Thank you for all the useful discussions \& the samenwerking. I had so much to learn 
about all those molecular aspects of HLA. Thank you as well for being my paranymph!

Students who worked with me at the lab: Jeroen, Nick, Heike, Paul, and Thara. It was nice to have you around and thank you for helping me with the experiments. (-) Heike, mijn ex-student en ex-buurvrouw. Haha.. het was leuk om je te leren kennen. Dank je wel voor je hulp, cookies, en de gezelligheid. Ik wens je heel veel success met je PhD! (-) Paul, the man with a good heart. Je hebt mij enorm geholpen met die macrofaag mega experimenten. Was een beetje te gek, he? (-) Thara, terimakasih banyak voor je hulp. Jij was echt een harde werker.

Our collaborators: $(-)$ at the department of Pathology - Dr. Myrurgia Abdulhamid, and the analists Cecilé, Carla, and Stefan thank you so much for your time and help with the ED-B staining. Unfortunately due to technical difficulties we had to discontinue the project $(-)$ at the department of Clinical Genetics - Dr. Johanna (Jannie) Janssen, and the analists, Rosie and Ruth thank you very very much for your help with the bone marrow samples. Jannie, it was a pleasure collaborating with you. Thank you for your enthusiasm, interest and dedication for our collaboration. (:) at Maastro lab - Dr. Ludwig Dubois \& Marike van Gisbergen, thank you for introducing me to Seahorse and for the helpful discussions. Dr. Kasper Rouschop, thank you for helping me with my hypoxia project and troubleshooting the machine(s) every now and then. (-) Jip Beugels, het was leuk om met je samen te werken aan de macrofaag experimenten! Succes met je opleiding! (-) at CDL - Dr. Monique Gromme, thank you very much for helping me with the marrow samples. $(-)$ Dr. Joris Vanderlocht, thank you for your input during lab meeting and the motivating discussions. All the best with your career!

My neighbors at the internal medicine lab: (-) José en Maria, dank jullie wel voor jullie hulp bij het lab en voor de gezelligheid (en cakejes). (-) Mitchell, jij hebt echt passie voor het wetenschappelijk onderzoek, succes met je post-doc en toekomst! (-) Kristiaan, het allerbeste met je wetenschappelijke carrière. (-) Montserrat \& Pan, thank you for your help and for lending me stuffs from your lab, as well as our chit chats.

My fellow GROW representatives: (-) Elke, Jules, Ghislaine, Eduardo, Cecile, het was heel leuk om met jullie samen te werken als GROW representatives. Dank jullie wel ook voor de gezelligheid (de etentjes en zo). Heel veel succes met jullie carrière. Jules, je bent echt een harde werker, heel gepassioneerd over je project, maar toch heb je genoeg tijd om actief te zijn bij verschillende organisaties. Ik bewonder dat! Eduardo \& Cecile, heel veel succes met je PhD en je taken als representatives! 
My teachers and seniors at the department of internal medicine, division of hematology \& medical oncology (Tulip Integrated Cancer Clinic) at the Universitas Gadjah Mada, Yogyakarta, Indonesia. Dr. Ibnu Purwanto, Sp.PD-KHOM, Dr. Johan Kurnianda, Sp.PD-KHOM, Dr. Kartika Widayati, Sp.PD-KHOM, Dr. Susanna H. Hutajulu, PhD, Sp.PD-KHOM, Dr. Mardiah Suci H., PhD, Sp.PD-KHOM. I cannot express how grateful I am for the opportunity you gave to me to work at Tulip. Without this, I will not be here today, writing my PhD thesis. I am eternally thankful for the valuable experiences I received during my work at Tulip. I hope that we can still collaborate together in the future despite the distance.

My former supervisors during my master study: (-) Dr. Joost Hegmans and Margaretha Lambers, Bsc. Joost \& Margaretha, I will always remember you both as the first persons who introduced me to laboratory science. Hartelijk dank voor jullie geduld en begeleiding tijdens mijn stage(s). Ik waardeer het enorm.

My former research master school: (-) Prof. dr. Anton Grootegoed, thank you for the opportunity you gave me to study at the molecular medicine research master program. It's a cornerstone of my scientific journey. (-) Benno Arentsen \& Marjolein van Berckel Bik, thank you for your help with the administration and social matters.

My dear friends: (-) Cynthia, Tamara, Lisanne, Bobby, Joyce, Anita, Rani, Fajar, Icha Triyanti, dank jullie wel voor de lieve vriendschap! Heel erg bedankt voor jullie steun. Ik weet dat ik op jullie echt kon rekenen $(-)$ en natuurlijk voor all de good times! (-) Yuda, we knew each other since we were studying medicine in Yogyakarta. I was really happy to have you around here in the Netherlands. You were such a kind-hearted and helpful person. Meeting you was always a pleasure. I was looking forward to our next one (October 2018) for your defense. But you didn't get the chance to defend your thesis. You went "home" too soon. Rust zacht, dear Yuda. Thank you for everything! You will always be in my heart and I will miss you a lot!

My dear family-in-law: (-) Mam, jij bent een hele lieve persoon en één van de meest positieve personen die ik ken. Heel erg bedankt voor je enorm steun en liefde. Ik ben heel gelukkig om jou te hebben als mijn schoonmoeder. (-) Rody, we zijn bijna op dezelfde tijd een PhD training begonnen. Tussen onze drukke PhD dagen door, hadden wij toch nog tijd om leuke dingen te doen in het weekend, ook met Sanne. Omdat je heel erg gepassioneerd over je project was, was ik altijd op de hoogte over CRISPR-cas9 in het weekend :P Maar, jij bent een geweldige zwager! Dank je wel voor de gezelligheid en je steun. Succes met de laatste loodjes van je PhD! 
My dearest family: (-)Dad, I always think you're awesome. I am so proud of you, of your achievements in your career. Although you are very successful in your field, you stay humble and compassionate. You will always be my inspiration and a true example of hard working and humility. I will always remember what you taught me "Do your best, work hard, don't whine, don't work solely for money, do your work with passion. Credits and compensation will follow..." You gave me something to dream on. Other 7 -years-old children might be dreaming of dolls, toys, or a prince charming, I dreamed of going school abroad, thanks to your bedside story about Harvard, Princeton, Yale, or Stanford. I haven't been to any of these schools yet, but perhaps one day.. I still keep dreaming. Thank you for your tremendous support, wisdom and love! I love you! (-) Mom, your sacrifice to stay at home to raise me and my brother, instead of chasing your own career, I don't think I could ever pay it back. I am forever indebted to you. While dad gave me something to dream on, you were actually the one who fueled my ambitions and dreams. You always cheered me up! You followed every single step I took to fulfill my dreams and tried to support me as much as you can. When I applied for this PhD vacancy, you were busy looking for places with a high-speed internet to facilitate my interview, since we had a bad internet connection at home. Thank you for always listening to me and believing in me. Thank you for your endless support, patience and love! I love you!! (-) My brother, to fullfil my dreams, you were kind of forced to follow me moving to Yogyakarta. I'm sorry for that. I would like you to know that I really appreciate it and I owe you my success. Thank you for your support and love! I wish you a lot of happiness with my sister in law, my niece and nephew.

My beloved son, $:$ Florian, your arrival in my last year $\mathrm{PhD}$ is a wonder. Your presence brought me an extra joy and spirit. There is no word can express how thankful and happy I am that God gave me you. Ik hou heel veel van je!

My dear husband and my best friend, (-) Timothy. With you I can share my thoughts, open up my heart, confide my feelings, and liberate my emotions. We always have a great communication and we understand each other, making the many kilometers between us in the beginning and the extra busy days in the last year, very doable. Thank you for always being there for me, for giving me strength, and believing in me. Ik kan je nooit genoeg bedanken voor je enorme steun, geduld, begrip, en liefde. Ik ben ontzettend gelukkig met jou! Ik hou heel veel van je!

Thank you. Dank je wel. Terima kasih.

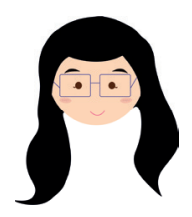

